Florida International University FIU Digital Commons

6-30-2010

\title{
Navigating Health Sources on the Internet: A Mixed-Methods Examination of Online Consumer Reviews and Expert Text on Psychotropic Drugs
}

Shannon Hughes

Florida International University, shughes5@msn.com

DOI: $10.25148 /$ etd.FI10080406

Follow this and additional works at: https:// digitalcommons.fiu.edu/etd

Part of the Medicine and Health Commons, Science and Technology Studies Commons, and the Social Work Commons

\section{Recommended Citation}

Hughes, Shannon, "Navigating Health Sources on the Internet: A Mixed-Methods Examination of Online Consumer Reviews and Expert Text on Psychotropic Drugs" (2010). FIU Electronic Theses and Dissertations. 231.

https://digitalcommons.fiu.edu/etd/231 


\section{FLORIDA INTERNATIONAL UNIVERSITY \\ Miami, Florida}

\section{NAVIGATING HEALTH SOURCES ON THE INTERNET: A MIXED-METHODS EXAMINATION OF ONLINE CONSUMER REVIEWS AND EXPERT TEXT ON PSYCHOTROPIC DRUGS}

A dissertation submitted in partial fulfillment of the requirements for the degree of DOCTOR OF PHILOSOPHY

in

SOCIAL WELFARE

by

Shannon Hughes 
To: Dean Fernando M. Trevino

Robert Stempel College of Public Health and Social Work

This dissertation, written by Shannon Hughes, and entitled Navigating Health Sources on the Internet: A Mixed-Methods Examination of Online Consumer Reviews and Expert Text on Psychotropic Drugs, having been approved in respect to style and intellectual content, is referred to you for judgment.

We have read this dissertation and recommend that it be approved.

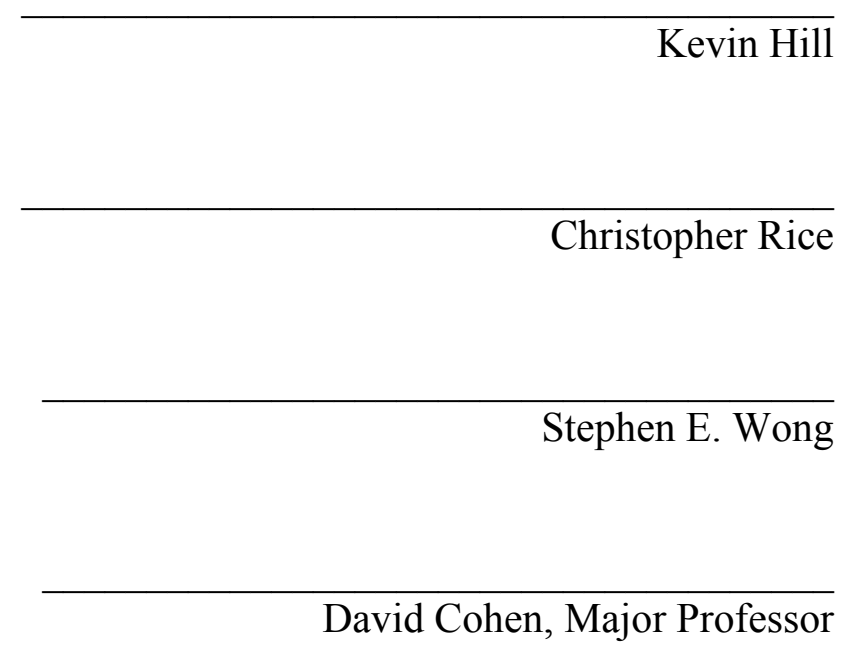

Date of Defense: June 30, 2010

The dissertation of Shannon Hughes is approved.

Dean Fernando M. Trevino Robert Stempel College of Public Health and Social Work

Interim Dean Kevin O'Shea University Graduate School

Florida International University, 2010 


\section{ACKNOWLEDGMENTS}

First, I thank my advisor, David Cohen, for his continuous support in the Ph.D. program. Every conversation with Dr. Cohen has heightened and renewed my enthusiasm for this work, even when I felt overwhelmed and defeated by the challenges of it. I have been constantly inspired by his thoughtfulness, creativity, and the perfection of his writing. Without his guidance and encouragement, I could not have finished this dissertation.

I would like to thank the rest of my committee members for their time and support throughout this process. I thank Dr. Christopher Rice for asking hard questions that pushed me to become a better thinker and researcher. I value Dr. Stephen Wong's insightful suggestions on each of my papers, and appreciate his encouragement of my work. I thank Dr. Kevin Hill for joining the committee so quickly and eagerly, and again appreciate the helpful comments.

During the course of this work, at Florida International University (2006-2009), I was supported by a graduate research assistantship with the School of Social Work. In 2009-2010, I was supported by the Florida International University Dissertation Year Fellowship. During that academic year, I was also awarded funds by the Fahs-Beck Fund for Research and Experimentation to cover certain research related expenses.

Let me also say thank you to the following people at Florida International University: Paul Stuart for being so attentive and responsive to doctoral student needs. Maria Gutierrez for helping me navigate through all the red tape and confusion related to getting enrolled, receiving paychecks, spending grant money, and so much more! Thanks to each of my professors during the first year of the program for being so understanding 
of the fact that we were new (and naïve) doctoral students. Juliette Graziano for being a remarkable cohort partner without whom I may not have made it through those first couple years. Juliette, I am so grateful that we entered and finished this together. I am also greatly indebted to my teacher of the past, Dr. Tomi Gomory, at Florida State University. Dr. Gomory profoundly influenced my thinking as an undergraduate and graduate student, and encouraged me to continue to the doctoral program. I would not be in this position today without his guidance. Thank you to Jeffrey Lacasse, formerly of Florida State University, for introducing me to this field as an undergraduate and for his patient advice since then.

Last, but not least, I thank my family and loved ones. My parents have always been unconditionally proud of me, and I thank them for allowing me to pursue my interests in any direction they took me. I thank them too for serving as a willing audience to listen to my frustrations and challenges throughout the program. My brother, Jason Hughes, reminds me that this work is an important and practical contribution to the world (just when I have seemingly forgotten). My dear friends, Kristina Obenza, Sarah Howey, and Denise Irwin have been wonderful in their support, and always there when I needed to relax and have fun. My partner in life, Louzonteno Johnson II, has been my rock for the last 4 years. I thank him for his unconditional support and love, and his unrelenting belief in me. This dissertation belongs to the both of us. 


\title{
ABSTRACT OF THE DISSERTATION \\ NAVIGATING HEALTH SOURCES ON THE INTERNET: A MIXED-METHODS \\ EXAMINATION OF ONLINE CONSUMER REVIEWS AND EXPERT TEXT ON \\ PSYCHOTROPIC DRUGS \\ by
}

\author{
Shannon Hughes \\ Florida International University, 2010 \\ Miami, Florida \\ Professor David Cohen, Major Professor
}

Purpose: The Internet has provided an unprecedented opportunity for psychotropic medication consumers, a traditionally silenced group in clinical trial research, to have voice by contributing to the construction of drug knowledge in an immediate, direct manner. Currently, there are no systematic appraisals of the potential of online consumer drug reviews to contribute to drug knowledge. The purpose of this research was to explore the content of drug information on various websites representing themselves as consumer- and expert-constructed, and as a practical consideration, to examine how each source may help and hinder treatment decision-making.

Methodology: A mixed-methods research strategy utilizing a grounded theory approach was used to analyze drug information on 5 exemplar websites ( 3 consumer- and 2 expertconstructed) for 2 popularly prescribed psychotropic drugs (escitalopram and quetiapine). A stratified simple random sample was used to select 1,080 consumer reviews from the websites $(\mathrm{N}=7,114)$ through February 2009. Text was coded using QDA Miner 3.2 
software by Provalis Research. A combination of frequency tables, descriptive excerpts from text, and chi-square tests for association were used throughout analyses.

Findings: The most frequently mentioned effects by consumers taking either drug were related to psychological/behavioral symptoms and sleep. Consumers reported many of the same effects as found on expert health sites, but provided more descriptive language and situational examples. Expert labels of less serious on certain effects were not congruent with the sometimes tremendous burden described by consumers. Consumers mentioned more than double the themes mentioned in expert text, and demonstrated a diversity and range of discourses around those themes.

Conclusions: Drug effects from each source were complete relative to the information provided in the other, but each also offered distinct advantages. Expert health sites provided concise summaries of medications' effects, while consumer reviews had the added advantage of concrete descriptions and greater context. In short, consumer reviews better prepared potential consumers for what it's like to take psychotropic drugs. Both sources of information benefit clinicians and consumers in making informed treatmentrelated decisions. Social work practitioners are encouraged to thoughtfully utilize online consumer drug reviews as a legitimate additional source for assisting clients in learning about treatment options. 


\section{TABLE OF CONTENTS}

CHAPTER

PAGE

1. STATEMENT OF PROBLEM

2. LITERATURE REVIEW 4

Definitions and Concepts $\quad 4$

Expert Drug Knowledge in the Off-line World 15

Consumer Drug Knowledge in the Off-line World 28

Expert and Consumer Knowledge in the Online World 31

Previous Forms of Internet Research $\quad 46$

Grounded Theory 49

Summary 51

Conceptual Approach $\quad 51$

Research Questions $\quad 56$

3. METHODOLOGY 58

Research Strategy $\quad 58$

The Sample $\quad 60$

Data Collection $\quad 70$

Data Analysis $\quad 75$

4. FINDINGS 86

Sample Characteristics $\quad 86$

Inter-coder Agreement Analysis 95

Drug Effects According to Consumers 99

Drug Effects According to Experts $\quad 115$

Drug Effects by Experts versus Consumers 123

Drug Effects by Variables 140

Themes Mentioned by Consumers 155

Themes by Experts versus Consumers 179

Usability of Consumer Sites According to Typical Web Use 192

Preliminary Data Checking 201

Data Checking of the Research Sample 205

5. STRENGTHS AND LIMITATIONS 215

Research Strategy 215

Authenticity of the Sample 216

Generalizability and Applicability 218

Units of Analysis 218

$\begin{array}{ll}\text { Statistical Analysis and Comparisons } & 219\end{array}$ 
6. DISCUSSION 222

Additional Data on Popularly Reported Drug Effects 222

$\begin{array}{ll}\text { Effect Classification } & 227\end{array}$

$\begin{array}{ll}\text { Variation among Consumers } & 229\end{array}$

How do Expert and Consumer Sources Help and Hinder Decision-Making? 231

$\begin{array}{ll}\text { Theoretical Explanations of Findings } & 237\end{array}$

$\begin{array}{ll}\text { 7. CONCLUSION } & 241\end{array}$

$\begin{array}{ll}\text { Hypotheses for Future Research } & 241\end{array}$

Future Research: Authenticity and Credibility 244

$\begin{array}{ll}\text { Future Research: Usability } & 245\end{array}$

Searching for Health Information on the Internet 248

LIST OF REFERENCES $\quad 251$

$\begin{array}{ll}\text { APPENDICES } & 266\end{array}$

$\begin{array}{ll}\text { VITA } & 353\end{array}$ 


\section{LIST OF TABLES}

TABLE

PAGE

3.1 Description of Website Typologies 61

3.2 Sampling Frame for Consumer Reviews (through February 2009) 74

3.3 Probability of Selection in a Stratified Simple Random Sample of 120 Cases per Drug per Website

4.1 Number of Consumers Reporting Gender across All Websites 88

4.2 Number of Consumers Reporting Gender According to Drug Taken 88

4.3 Number of Consumers Reporting Age across All Websites 89

4.4 Number of Consumers Reporting Age According to Drug Taken 90

4.5 Number of Consumers Reporting Length of Time on the Drug across All Websites

4.6 Number of Consumers Reporting Length of Time on the Drug according to Drug Taken

4.7 Number of Consumer Posts According to Year Posted across all Websites

4.8 Number of Consumer Posts for Each Drug According to Year Posted

4.9 Number of Consumers Who Provided a User ID or Email Address across All Websites

4.10 Number of Consumers Who Provided a User ID or Email Address According to Drug Taken

4.11 Inter-coder Agreement Analysis

4.12 Top 5 Effect Categories Mentioned by Consumers for Lexapro

4.14 Number of Consumers on each Website Mentioning the Most Popularly Reported Effects of Lexapro 
4.15 Lexapro Effects Mentioned by $>3 \%$ of Consumers according to Website Classification

4.16 Top 5 Effect Categories Mentioned by Consumers for Seroquel

4.17 Effects of Seroquel Mentioned by at Least 3\% of Consumers

4.18 Number of Consumers on Each Website Mentioning the Most Popularly Reported Effects of Seroquel

4.19 Seroquel Effects Mentioned by $>3 \%$ of Consumers According to Website Classification

4.20 Most Frequently Mentioned Effects of Lexapro in Expert Text (frequency $>3 \%$ of total mentions of effects on either website)

4.21 Most Frequently Mentioned Effects of Seroquel in Expert Text (frequency $>3 \%$ of total mentions of effects in either website)

4.22 Excerpts from Experts and Consumers on Selected Mental/Mood Effects of Lexapro

4.23 Comparison of Experts and Consumers on Appetite and Weight Effects of Lexapro

4.24 Comparison of Experts and Consumers on Sexual Effects of Lexapro

4.25 Excerpts from Experts and Consumers on Brain Fog/Zombie Effect of Seroquel

4.26 Comparison of Experts and Consumers on Sleep Effects of Seroquel

4.27 Excerpts from Experts and Consumers on Selected Sleep Effects of Seroquel

4.28 Comparison of Experts and Consumers on Lab Tests and Chronic Conditions Mentioned for Seroquel

4.29 Gender of Consumers who Mentioned an Effect of Lexapro According to Drug Effect Category 
4.31 Male and Female Consumers Compared to Those Who Did Not

Report Gender on Drug Effects for Selected Categories of Lexapro

4.32 Age of Consumers Who Mentioned an Effect of Lexapro

According to Drug Effect Category

4.33 Reporting of Age by Drug Effect Category for Lexapro

4.34 Consumers Taking Lexapro According to Length of Time Taken and Drug Effect Category

4.35 Reporting Length of Time Taking Lexapro According to Drug Effect Category

4.36 Gender of Consumers Who Mentioned an Effect of Seroquel According to Drug Effect Category

4.37 Reporting Gender by Drug Effect Category for Seroquel

4.38 Age of Consumers Who Mentioned an Effect of Seroquel According to Drug Effect Category

4.39 Reporting Age by Drug Effect Category for Seroquel

4.40 Consumers Taking Seroquel by Length of Time Taken and Drug Effect Category

4.41 Reporting Length of Time Taking Seroquel by Drug Effect Category

4.42 Frequency of Consumers Within Each Website Who Mentioned a Code Within the Category Assessing the Overall Experience

4.43 Number of Consumers Mentioning Codes under Assessing the Overall Experience, According to Website Classification

4.44 Frequency of Consumers Within Each Website Who Mentioned a Code Within the Category Dealing with Side Effects/Finding Optimum Effectiveness, Mentioned by at Least 3\% of Consumers on Any Website

4.45 Codes Under Dealing with Side Effects/Finding Optimum Effectiveness that Reached Statistical Significance $(p<.05)$ According to Website Classification 
4.46 Frequency of Consumers Within Each Website Who Mentioned a

Code Within the Category Do I Need Meds

164

4.47 Number of Consumers Mentioning Codes Under Do I Need Meds

According to Website Classification

4.48 Frequency of Consumer Comments on Drug Theme Categories (in bold text) and the Most Frequently Mentioned Code Within that

Category (in plain text)

4.49 Number of Consumers Mentioning Codes under Other Drug

Theme Categories According to Website Classification

4.50 Frequency of Consumers Within Each Website Who Mentioned a Code Within the Category My Doctor, Mentioned by at Least $3 \%$ of All Consumers

4.51 Number of Consumers Mentioning Codes under My Doctor According to Website Classification

4.52 Frequency of Consumer Comments on Other Theme Categories (in bold text) and the Most Frequently Mentioned Code Within that Category (in plain text)

4.53 Codes From All Theme Categories That Were Mentioned in Expert Text

4.54 Number of Consumer and Expert Cases that Mention a Code Under Assessing the Overall Experience

4.55 Expert and Consumer Descriptions of Assessing the Overall Experience

4.56 Consumers and Experts on the My Doctor Code: Talking with Doc/Doc Explained/Consult Doc

188

4.57 Excerpts from Consumers and Experts on My Doctor

4.58 Most Frequently Mentioned Effects of the Most Recent 20 Consumer Posts From Each Website Compared to All Consumer Posts for Lexapro

4.59 Less Frequently Mentioned Effects from Recent Posts Compared to the Full Dataset for Lexapro 
4.60 Most Frequently Mentioned Effects of the Most Recent 20 Consumer Posts From Each Website Compared to All Consumer Posts for Seroquel

4.61 Less Frequently Mentioned Effects of Recent Posts From Each Website Compared to the Full Dataset for Seroquel

4.62 Most Frequently Mentioned Themes of Most Recent 20 Posts From Each Website Compared to the Full Dataset of Both Drugs

4.63 Data Checking by Time Period of Consumers' Posts and Drug Effect Categories for Lexapro and Seroquel Combined

4.64 Data Checking by Time Period of Consumers' Posts on Selected Code Categories for Both Drugs Combined

4.65 Data Checking for Anonymity of Users by Drug Effect Categories for Lexapro and Seroquel Combined

4.66 Data Checking for Anonymity of Consumers on Statistically Significant Code Categories for Both Drugs Combined

4.67 Data Checking for Anonymity of Consumers on Selected Mental or Mood Effects for Lexapro and Seroquel Combined 


\section{LIST OF FIGURES}

FIGURE

PAGE

2.1. Traditional and Evolving Dynamics in the Production of Psychotropic Drug Knowledge

4.1 Most Popularly Mentioned Lexapro Effects by

Consumers across Websites

4.2 Most Popularly Mentioned Seroquel Effects by

Consumers across Websites

4.3 Top 6 Most Frequently Mentioned Lexapro Effects in Expert Text Measured by Number of Mentions

4.4 Top 6 Most Frequently Mentioned Lexapro Effects in Expert Text Measured by Proportion of All Mentions of Effects

4.5 Top 6 Most Frequently Mentioned Seroquel Effects in Expert Text Measured by Number of Mentions

4.6 Top 6 Most Frequently Mentioned Seroquel Effects in Expert Text Measured by Proportion of All Mentioned of Effects

4.7 Comparison of Frequency of Mentions of Lexapro Effects between Experts and Consumers, by Effect Category

4.8 Comparison of Most Frequently Mentioned Lexapro Effects between Experts and Consumers

4.9 Comparison of Frequency of Mentions of Seroquel Effects between Experts and Consumers, by Effect Category

4.10 Comparison of Most Frequently Mentioned Seroquel Effects between Experts and Consumers

4.11 Proportion of Mentions of the Most Popular Themes by Experts and Consumers, Respectively, Out of Total Mentions of All Themes

4.12 Proportion of Mentions of the Most Popular Codes Within the Category Dealing with Side Effects/Finding Optimum Effectiveness Out of Total Mentions Within the Category 


\section{Chapter 1: Statement of Problem}

The central issue examined in the proposed research concerns the construction of expertise in the age of the Internet. To address the central issue, this study focuses on an area - psychotropic drugs prescribed and used as medicines - to examine evolving relations of power in the production and dissemination of knowledge in today's prescription drug market.

Expert knowledge of psychotropic drugs is that which is produced using procedures of science, and represents the official account of drugs' uses and effects. The forthcoming review of literature will show, however, that this scientifically-derived expert knowledge has become highly contested. The blurring lines between pharmaceutical industry marketing and scientific activities, and the near monopoly of the industry over production and dissemination of expert/scientific knowledge of psychotropic drugs has resulted, at a minimum, in well-documented publication biases and considerable uncertainty about drug efficacy and safety (Huston \& Moher, 1996; Ioannidis \& Lau, 2001; Melander, Ahlqvist-Rastad, Meijer, \& Beermann, 2003; Perlis et al., 2005; E. Turner, Matthews, Linardatos, Tell, \& Rosenthal, 2008). Also, to reach a "valid" understanding of drugs' effects, researchers have persistently muted the direct voice and speech of the patient in favor of a presumably more objective (biomedical, scientific, statistical) discourse (Jacobs \& Cohen, 1999).

In parallel, the Internet allows researchers, clinicians, and consumers to review drug experiences and information from thousands of consumers without professional mediation, and presumably without pharmaceutical industry supervision over content. The Internet thus makes more apparent multiple and sometimes contradictory claims to 
knowledge. How these less scientific ways of knowing about psychotropic drugs made accessible through the web contribute to or dilute the expert/scientific knowledge base, or how they constitute their own knowledge base, remains virtually unexplored.

Moreover, "democratizing" the production of knowledge and consumers' broadened acceptance of what constitutes legitimate, trustworthy, or credible information results in a trans-disciplinary debate about the appropriate role of experts and non-experts in policy, media, science, and culture, as well as the true nature of what is being applauded as "democratization" (Dahlberg \& Siapera, 2007; David, 2007; Keen, 2007; Siegel, 2008). Applied to the construction of knowledge about psychotropic drugs, the fundamental pragmatic concern of this debate is whether expert knowledge on psychotropic drugs is sufficient for building a knowledge base and helpful to those who believe they need expertise to come to an understanding or decision about a drug issue, or whether expertise is a shroud that actually limits or distorts the knowledge base and disadvantages those seeking expertise because of conflicts of interest and other biases. Has the Internet rendered expertise in the area of psychotropic drugs obsolete?

Following from this, the main question of the research was: Does the accumulated consumer or layperson knowledge that the Internet allows dissolve the traditional boundary between expert/scientific and consumer/lay knowledge? The research specifically sought to examine the content, in terms of drug effects and themes, of drug information on consumer-constructed and expert-constructed web sources. A grounded theory approach as part of a mixed-methods research strategy was used to analyze the content of five websites containing drug information for two popularly prescribed psychotropic drugs from different drug classes. As a practical consideration, how does 
each source help and hinder treatment decision-making for clinicians and for actual and potential consumers? 


\section{Chapter 2: Literature Review}

\section{Definitions and Concepts}

\section{Psychotropic drugs.}

Psychotropic drugs of relevance in the present analysis are those drugs available by prescription, consumed as medicine, and intended to alter the behavior, mood, thinking, and/or feeling of the individual ingesting the drug. Psychotropic drugs can be analyzed on numerous levels, such as for their effects on biological processes or their personal, social, cultural, political, and economic meaning and symbolism, and each level of meaning or understanding interacts with drug effects (Cohen, McCubbin, Collin, \& Perodeau, 2001). In the marketplace, a psychotropic drug may be considered an “experience good," meaning a good whose value or quality cannot be known until the good has been personally experienced (Azoulay, 2002). In Foucauldian analysis, psychotropic drugs may be considered technologies of the body that carry disciplinary messages of transformation and self-surveillance to the consumer (Clarke, Mamo, Fishman, Shim, \& Fosket, 2003). The proposed research does not reduce psychotropic drugs to a single defining quality or use, but rather acknowledges that many ways of understanding drug use or effects may be equally valid.

That being said, there are some pharmacological basics that may elucidate the nature of drug knowledge. First, a basic tenet of pharmacokinetics is that the blood or plasma concentration of drugs is related to drugs' effects, but for most psychotropic drugs, no clear or simple relationship has been found (Brunton, Lazo, \& Parker, 2006). In trials of antidepressants, for example, "such a high proportion of patients respond to placebo that it is difficult to determine the plasma level associated with efficacy" ( $p$. 
128). Second, at the population level, therapeutic dose ranges for all drugs will overlap with toxic dose ranges. This is also true at the individual level for antipsychotic drugs, which are typically selected based on the likely tolerability of adverse effects.

Complicating all of this, a single individual may not always respond the same way to the same drug given at different times. Third, psychotropic drugs primarily act initially on the transport of specific neurotransmitters, but cause a cascade of secondary effects and adaptive processes that still elude researchers.

In sum, much is still not known physiologically about how, why, or for whom psychotropic drugs "work." Drugs have multiple effects, which are unpredictable for individuals and too complicated for current scientific processes to tease apart. However, even if there did exist a "perfect" knowledge of the physiology of drug action, it would be an incomplete knowledge because drugs' effects are as much social phenomena as they are biological (Cohen et al., 2001).

Knowledge.

Methods and procedures of science are widely accepted as producing - or at least of providing the optimum means of progressing towards - what may be called "true" knowledge characterized by objectivity and neutrality. It is arguable, however, that knowledge produced by scientific methods (henceforth, scientific knowledge) is incomplete, limited, and not free from bias due to cultural and political influences. The alternative, critical hypothesis that informs the proposed research posits that knowledge takes various forms, may be dispersed in an incomplete and often contradictory manner among individuals, and cannot be concentrated, organized, integrated, or "knowable" by any one or elite group of individuals (Foucault, 1980; Hayek, 1945). Central to the 
research is the best utilization of dispersed knowledges, a position which may consequentially suspend oppositions between scientific and non-scientific knowledge, or objective and subjective ways of knowing.

This paper also adopts Michel Foucault's concept of knowledge as inextricably and inevitably linked to power (power/knowledge) (Foucault, 1980). According to Foucault, power is dispersed, exercised from innumerable points, and refers to relations rather than a possession or privilege of a dominant class. Power may be repressive, but is more often productive by, for example, producing knowledge and discourses which are exercised at the level of everyday life in activities like self-surveillance and self-discipline. Foucault describes:

there is no power relation without the correlative constitution of a field of knowledge, nor any knowledge that does not presuppose and constitute at the same time power relations... It is not the activity of the subject of knowledge that produces a corpus of knowledge, useful or resistant to power, but power-knowledge, the processes and struggles that traverse it and of which it is made up, that determines the forms and possible domains of knowledge. (Rabinow, 1984, p. 175)

The effectiveness of the positive, productive characteristics of power rests on the installation of a field of empirical truth, or what Foucault terms a politics or regime of truth (Foucault, 1980). The politics of truth encompasses: the types of discourse a society accepts and makes function as true, the mechanisms used to distinguish true from false statements, the procedures accorded value for acquiring truth, and the status afforded to those authorized to say what counts as true. The analysis of power/knowledge, then, is 
not concerned with discerning true from false statements, but rather stresses the historical conditions of possibility for what counts as truth. In the current politics of truth, the procedures and practitioners of science hold great currency. As a result, seemingly natural objects (like disease or schizophrenia) are best understood only in relation to the science or expertise that posits them, as "the very discourse that is supposed to discover the reality of the thing's being, the truth of disease itself..., has always already actively contributed to the constitution of the object" (Rawlinson, 1987, p. 377). If scientificmedical knowledge appears neutral in medical discourse (i.e., what is said by those speaking as medical experts), it is because the knowledge offered is constructed as external to power (Peterson \& Bunton, 1997). Thus, while it is mostly inaccurate to construe medical experts as figures of domination who possess power that could be given to someone else, it is accurate to say that they are important links in a set of power relations capable of producing knowledge.

Expertise and the role of experts in society.

Political rule in liberal societies has greatly depended on experts and the authority of truth to govern populations (Rose, 1993). Experts are authorized to construct knowledge around an identified object, and political rule is exercised in light of the knowledge of that which is to be governed. The expert knowledge is not typically a new knowledge or expertise over an object where no knowledge or expertise had existed before, but rather often represents a reorganized, reformatted, and concentrated form of a local, dispersed, or subjugated knowledge (Mitchell, 2002). Thus, experts are those who can speak and enact truth, and serve as the means of circulation of this reorganized and concentrated knowledge. 
In advanced liberal societies, such as presently in the U.S. according to many social scientists, experts are increasingly distanced from overt political rule through a shift in location of experts and expert knowledges to the market governed partly by consumer demand (Rose, 1993). Advanced liberal rule depends on the self-government of individuals "whereby each individual binds themselves to expert advice as a matter of their own freedom... what starts off as a norm to be implanted into citizens can be repossessed as a demand which citizens can make of authorities" (p. 296). As opposed to the use of coercive or repressive techniques based on expert knowledge of a human subject or population, advanced liberal societies seek to govern through regulated spheres of autonomy in which an individual's relation to oneself is constituted and shaped by a proliferation of discourses and technologies.

Expertise in areas of medicine, law, science and similar professions has traditionally emerged in society through the institutionalization of a field of knowledge, setting up of professional bodies, training and accreditation, and other techniques for regulating and managing the respective knowledge and activities (Abbott, 1988). This expert knowledge, though, is a contested knowledge in that there are always parallel disagreements and struggles both within expert circles and from the outside margins (such as that of the ill person or psychiatric patient) (Foucault, 1980). Constituting the outside margins are the local, particular, subjugated knowledges which have been "disqualified as inadequate to their task or insufficiently elaborated; naïve knowledges, located low down on the hierarchy, beneath the required level of cognition or scientificity" (p. 82). According to norms of science (and the current politics of truth) such knowledge may be considered either a flawed form of knowledge or something 
other than knowledge altogether. Social studies of health and illness have often used the term "lay beliefs" in reference to local, particular experiences, perspectives, meanings, and ways of managing health and the body, and have only recently accorded these the status of "lay knowledge" (Popay \& Williams, 1996). Alternatively construed, that which may be or become subjugated knowledge often involves an expertise (local and particular) too widely dispersed to provide a means for political power or corporate profits (Mitchell, 2002). This knowledge contains the "historical knowledge of struggles" and helps to reveal the contestations, multiple claims, local variations, and sometimes violence in the politics of truth (Foucault, 1980, p. 83). For the present analysis, to operationally define or label what knowledge $i s$ would entail "settling on paper issues that are not settled in reality, where they are the stake of ongoing social struggles" (Bourdieu, 1988, p. 778). Reference throughout this paper is therefore simply made to scientific knowledge and consumer knowledge - the former referring to that which is accepted as true using procedures of science (often called "knowledge" or "fact"), and the latter referring to that which holds less significance in determining what is accepted as true (often called "belief" or "opinion").

\section{Evolving market conditions.}

The individual and collective health of bodies in the U.S. is a political and economic issue because health, reproduction, and disease are central to economic processes and therefore subject to political control. Evolving market conditions, including medicalization, consumerism, direct-to-consumer advertising of prescription drugs, and the user-generated Internet, continually alter and transform power relations surrounding access to and demand for knowledge, and power over its production. 
Medicalization refers to the increasing number of life and bodily experiences that are placed within medical jurisdiction and subject to technical solutions (Conrad, 2007). Consumerism refers to the increased responsibility citizens carry to choose health goods and services through the marketplace (Gabe \& Calnan, 2000). Unique to the health domain, this form of consumerism involves an end user who does not necessarily "choose" the good since this decision may be mediated by the doctor, and who does not usually directly purchase the good because of health insurance and third-party payments (Busfield, 2006; Lakoff, 2004). Nevertheless, health in modern America is promoted and perceived as a commodity under which everyday lifestyle choices are subsumed, and health care is increasingly subject to consumer market forces (Conrad, 2005; Lewis, 2006). This consumerist trend, however, is less likely to apply to certain populations, such as children receiving Medicaid benefits or adults institutionalized in long-term care facilities. In this paper, the term "consumer" is meant to apply to a participative adult population. The commodification of health and increased responsibility put on consumers to "take control" of their care and treatment, for example by gaining medical knowledge, combines with medicalization to extend self-care and self-discipline into more and more corners of individuals' daily lives (i.e, through self-surveillance, risk assessment, consumption of appropriate goods/services for treatment/prevention of various problems) (Clarke et al., 2003).

Direct-to-consumer advertising (DTCA) of drugs available only by prescription has helped fuel both consumerism and medicalization by motivating individuals to proactively seek a drug, as well as more generally expanding the range of human distresses presumed amenable to drug treatment (Davis, 2006; McCurdy, 2003). As 
testimony to the former, one estimate found that nearly 8.5 million Americans each year request and receive a drug that they heard about or saw in an advertisement (McCurdy, 2003). Between half and three-quarters of consumer requests for a specific or general drug for depression were complied with by physicians according to one randomized controlled trial (Kravitz et al., 2005). DTCA is intended to blur the boundaries between mild and severe distress and to suggest that all degrees of suffering - or even a lifestyle wish that causes no apparent suffering - are amenable to drug treatment (Davis, 2006; Gilbert, Walley, \& New, 2000). The life one wants, that is, to be thinner, happier, more productive, more confident, better liked, and better rested, is promoted as a doctor visit away. This brand of "cosmetic psychopharmacology" has been particularly successful for easily self-diagnosed problems such as for weight, baldness, and erectile dysfunction, as well as for shyness and other behavioral, performance or personality difficulties (Applbaum, 2006; Avorn, 2005; Conrad \& Leiter, 2004; Lexchin, 2006).

Pharmaceutical companies may also use DTCA to market a disease to build awareness of an underutilized psychiatric diagnosis and subsequently sell the drug to treat the disease (Conrad, 2005). "Mood stabilizer" drugs, for example, had scantly been discussed until after 1995 when the anti-convulsant drug, Depakote, was approved by the FDA for treating mania (Healy, 2006a). The campaign for Bipolar Disorder and mood stabilizers involved DTC television and magazine advertisements, popular media coverage such as a 2002 Time magazine cover story titled Young and Bipolar, new medical journals devoted to the disease, drug company-funded online patient groups, Internet sites, and scientific and medical conferences. Pediatric Bipolar Disorder, an 
extremely rare diagnosis 10 years ago, has today an estimated prevalence among youth reaching up to 1 million (Smarty \& Findling, 2007).

Direct-to-consumer advertising, as part of the overall phenomena of medicalization and consumerism, has served to transform relationships between product and consumer, and consumers and themselves. Paradoxically, the shift of the site of responsibility for screening and managing health from the doctor's office to the home of the consumer increases while it transforms and disperses the processes of medicalization (Clarke et al., 2003). The ongoing maintenance of health has become an individual goal, while the realm of what is considered "health" expands to include nearly any lifestyle choice, and consumer demand for health knowledge and technologies increases.

\section{The Internet.}

The Internet has created conditions for access to knowledge from multiple sources with sometimes contradictory messages, and carries the potential to alter power relations involving the production of what is deemed legitimate health (including psychotropic drug) knowledge. According to a Harris Interactive Poll, 110 million Americans use the Internet at least sometimes to seek health information (Taylor \& Leitman, 2002). This figure represents $80 \%$ of all adults online and $53 \%$ of all adults in the population. A 2008 survey reported the Internet to be the most widely used resource by adults for finding health information, though doctors were the most trusted source for information (Elkin, 2008). Thirty-four percent of online health searchers reported visiting Wikipedia, message boards, and discussion forums, mostly to find or exchange information about a specific disease, condition, or treatment. Use of user-generated online content is particularly prominent among consumers age 18 to 34 and those with at least some 
college education. A group of about 10 million Internet users regularly post health information online, and nearly 9 million users visit personal blogs for health information ("Today in eHealth news: Heard on the street," 2007, January 4). With a full 23\% of Americans without access to the Internet, it is accurate to assert that only a sub-set of adults use and contribute to the user-generated Internet, suggesting that its role and effects in health knowledge production and dissemination have only begun to develop. Still, media commentators insist that the cutting edge of today's evolving market is that "the rise of consumerism across American society today has positioned the consumer as king and the Internet as queen in the realm of healthcare communications" (O'Neill, 2007, p. 13).

The openness of the Internet highlights the always existing tension between expertise and democracy, and appears to swing the pendulum towards the extension of democratic participation (in the sense that the system is open to non-experts) while shrinking the perceived need or value of, and possibly attempting to redefine, expertise. Public participation in areas of science, medicine, and technology, especially as they intersect with morals and ethics, may be offered as a solution to the "problem of legitimacy," or crisis of confidence, in these fields (Collins \& Evans, 2007). The growing use and participation on the Internet has helped create a parallel "problem of extension," referring to the problem of identifying the point at which public involvement should be curtailed in order to maintain the presumably necessary boundary between expert and lay knowledge.

Some social commentators take the position that the abundance and variety of health information available through the Internet makes it ever more necessary to rely on 
expert-provided disciplined analysis and understanding to decipher truth from non-truth (Collins \& Pinch, 2005; S. Turner, 2001). The Internet is described as a chaotic mess of anonymous, uninformed, and unaccountable amateur opinions and claims that crowd thoughtful analysis by trained, qualified, and accountable experts (Keen, 2007; Siegel, 2008). This perspective assumes the traditional criterion of qualifications or credentials for judging expertise (Collins \& Evans, 2007). Too much “democratization” of knowledge to an unqualified lay public may have serious adverse social consequences as highly valued concepts of truth, reliability, and accountability become obscure or empty.

An alternative view of the Internet's potential effects posits that the Internet is "democratizing" only as much as it allows marginalized discourses - those that only weakly influence the public sphere - "to develop their own deliberative spaces...; to link up with other excluded voices...; and subsequently to contest those meanings and practices dominating mainstream public spheres" (Dahlberg \& Siapera, 2007, p. 134). Monetary requirements to ensure search engine optimization and extensive hyperlinked networks make it more likely that dominant and institutionalized discourses are codified "as so-called quality and thus trusted" (Dahlberg, 2005, p. 166). Rather than a fear of expert knowledge being drowned in a sea of ill-considered lay opinions, then, this perspective emphasizes that expert knowledge continues to dominate discourse on the Internet through the same corporate and professional influences that permeate off-line realms of knowledge production and dissemination. Thus, "democratization" of marginalized and counter discourses may need to be strategically fostered, rather than limited or regulated, on the Internet. 


\section{Expert Drug Knowledge in the Off-Line World}

\section{Expert (scientific/medical) drug knowledge.}

Discourse serves to constitute the subjects to whom and of whom it speaks, as well as to create conditions for what is possible and acceptable to say or claim as true (Rabinow, 1984). In line with this function, a scientific biomedical discourse has shaped the definition and treatment of a range of human maladies and distresses over the last 50 years (Dixon-Woods, 2001; Gabe, Kelleher, \& Williams, 1994). This discourse has thrived in tandem with cultural expectations and health policies that promote an ideal of a healthy and productive citizen (Gardner, 2003), as well as professional and regulatory demands that prescribed drugs be disease-targeted and tested for efficacy with specific scientific methodologies (Lakoff, 2004). For psychiatry, in particular, "the financial muscle of the pharmaceutical industry has helped to favor a predominantly biological view of psychiatric disorder," which has driven psychiatric treatment and the research agenda (Moncrieff \& Thomas, 2002, p. 216).

Expert knowledge about psychotropic drugs is primarily produced using procedures of science, exemplified by the randomized controlled clinical trial. The resulting knowledge is officially disseminated through the FDA-approved drug label and funneled to practicing clinicians through publication of trial results in medical journals, the Physician's Desk Reference (PDR), pharmaceutical representatives and medical education venues. However, significant conflicts of interest posed by pharmaceutical industry activity in both science and marketing threaten the integrity of the "expert" evidence base (Cohen, 2005; Healy, 2006b; Woosley, 1994). Pharmaceutical companies do not only manufacture the promoted drug, but, it will be shown, also produce and 
disseminate the information to be used by clinicians and consumers to make treatment decisions. This problem is not new or unknown to researchers - and perhaps also to clinicians and consumers - as researchers on many occasions have suggested the "manipulation of medical knowledge by the pharmaceutical industry...is endemic in psychiatry" (Moncrieff \& Thomas, 2002, p. 216).

Procedures of science in the making of drug knowledge.

Scientific procedures and regulatory demands for drug approval have combined to make certain requirements, termed in phases of drug testing, for producing valid knowledge about a drug. Phase III trials are intended to provide the most definitive efficacy data and additional safety data once the drug completes pre-clinical through Phase II testing. These efficacy trials take the form of short-term (usually 3-8 weeks), double-blind, randomized, controlled studies (RCT), which are presumed - assuming homogeneity of patients and their proper randomization - to generate causal links between treatment and outcome. The RCT format has trumped other methodologies as the "gold standard" for ascertaining a treatment's efficacy (Cohen, 2005; Oates, 2006). In psychopharmacology, however, the procedures of the RCT may be misapprehended and misapplied in several ways.

First, as opposed to other areas of medicine in which objective pathologies of the body are amenable to technological intervention and monitoring, psychiatry and psychopharmacology have no standard other than the behaviors listed in the Diagnostic and Statistical Manual of Mental Disorders (DSM) to identify what is being treated. The creation of diagnostic categories in the DSM can be likened to an opinion poll among experts, as the authors of the text clearly suggest: "Before DSM-IV, diagnostic manuals 
were generally constructed by expert consensus" (American Psychiatric Association, 1998, p. 1). Revisions to the current manual were mandated by the DSM-IV task force to be "data based," which paradoxically institutionalized the consensus-derived categories of previous editions while allowing the text to now be promoted as empirically informed (Kutchins \& Kirk, 1997). DSM authors further state that the manual's categorical distinctions between disorders cannot be supported logically (American Psychiatric Association, 1994), thus begging the question of what exactly is being treated in a psychopharmacological RCT (Cohen \& Jacobs, 2007)?

Correlatively, the determination of a psychiatric diagnosis and drug treatment effectiveness is unique because they are each based on the person's subjective complaints and feelings, as well as the doctor's observation of the person's behavior as in accordance or not with what the person expresses (Freund \& McGuire, 1999). However, the RCT format effectively mutes the participant's voice in favor of the investigator's voice through the use of investigator-rated measurements, narrowly defined outcomes which are of medical/professional interest, limited or no opportunities for unstructured or spontaneous speech by the participant, and limited interaction between the participant and investigator (Jacobs \& Cohen, 1999). Conventional symptom-based checklists and laboratory tests for biological effects (i.e., heart rate, blood pressure, plasma concentration) that are widely used in trials for medical products are only minimally relevant to psychopharmacology.

Finally, RCT criteria to ensure internal validity and rigorous testing appear to be misapplied or misapprehended in psychopharmacology trials. For example, placebos have demonstrated considerable efficacy - often equivalent or superior to the treatment 
drug - in psychopharmacology (Kirsch, Moore, Scoboria, \& Nicholls, 2002), yet this response receives little serious discussion in the literature with most researchers viewing it as a methodological problem that "complicates clinical trials of efficacy" (Oates, 2006, p. 118). The placebo response is further obscured in drug trials through the frequent use of inert placebos and abrupt drug discontinuations (Cohen \& Jacobs, 2007). The doubleblind is used in RCT procedures to minimize the bias of expectation and suggestion, though rarely do studies report methods for ensuring or testing maintenance of the blind. Use of inert placebos may further contribute to penetration of the blind in psychopharmacology trials in which active drugs nearly always have noticeable adverse effects. Third, RCT procedures for assessing adverse effects are underdeveloped and receive little attention compared to methods for measuring and reporting efficacy outcomes (Cohen, Hughes, \& Jacobs, in press; Greenhill et al., 2003). The most frequently used methods for collecting information on adverse effects involve "openended questioning" and "unsolicited reports" of the patient, which undoubtedly underestimate the frequency of many events and may mask the severity of others. Other methodological limitations of the RCT as used in psychopharmacology include: inattention to implications of widespread polypharmacy on drug efficacy and effects, discontinuation designs that may be confounded by drug withdrawal effects, failure to adequately recognize drug withdrawal effects, lack of post-treatment follow-ups of the previous drug experience, insufficient consideration of non-surrogate measures such as social and functional outcomes, and a general neglect of the psychoactive nature of psychotropic drugs (Charlton, 2001; Cohen \& Jacobs, 2007; Lagomasino, DwightJohnson, \& Simpson, 2005; Moncrieff, 2001). All of the above outlined factors combined 
provide legitimate grounds for contesting the validity of the primary means of production of expert drug knowledge.

Nevertheless, the information derived from pre-clinical through Phase III research is negotiated between the FDA and sponsoring pharmaceutical company and serves as the basis for the drug product label (Murphy \& Roberts, 2006). According to FDA representatives, the drug label is "the closest one can get to the truth regarding the scientific information known about a drug" (p. 36), and represents the "formal, government-approved definition of a drug's benefits and risks" (Avorn, 2006, p. 2409). However, litigation has revealed negotiations between the FDA and a pharmaceutical company spanning 5 years "over changing the drug's label to include adverse event data that had been submitted to the agency but not made fully available to the public" (Kesselheim \& Avorn, 2007, p. 310). The FDA has consistently supported the argument that a pharmaceutical company should not be expected to provide adverse event information other than that required to be printed in the drug label. Except for these few cases in which documents are revealed as part of litigation, it remains largely unknown what information in the drug label remains absent, minimized or reconfigured as a result of the negotiation process.

Information about a drug's full effects, which remains incomplete upon initial drug approval, is theorized to eventually emerge through long-term use in the general population. Phase IV post-marketing trials are deemed necessary to examine a drug's long-term effectiveness, rare and distal adverse effects, withdrawal effects, costeffectiveness, the impact of a drug on quality of life, and other important clinical outcomes (Corrigan, 2002). However, as of 2006 , at least $71 \%$ of post-marketing studies 
that drug companies committed to conducting when their product was approved by the FDA remain uncompleted or never started (Avorn, 2007). Post-marketing surveillance systems, such as the FDA's MedWatch, are under-utilized, tedious to complete, and subject to many of the same inadequacies in data collection identified above with clinical trial research (Kessler, 1993; Medawar \& Herxheimer, 2003). Information that is theorized to emerge from wide-spread drug use is not reliably collected nor promptly added to the drug's officially recognized profile (the drug label) (Avorn, 2006; Kesselheim \& Avorn, 2007).

Pharmaceutical company influence in scientific drug knowledge production. An issue that magnifies methodological deficiencies of psychopharmacology trials and lack of regulatory enforcement by the FDA is the fact that the pharmaceutical industry supports $70 \%$ of all costs of clinical trial research in the United States, and contributes over half of the budget for the Center for Drug Evaluation and Research through user fees (Avorn, 2007). Frequently, large Phase III RCTs occur in the context of multi-site, often multi-country trials conducted by contract research organizations (CROs) that are hired by the sponsoring pharmaceutical company (Relman \& Angell, 2002). Medical education and communication companies (MECCs) are hired to "ghostwrite" and strategically plan the resulting publications, while the pharmaceutical company's marketing department coordinates the process from beginning to end (Healy, 2004; Sismondo, 2007). This entails deciding which studies to pursue and for what indication, who a publication should be authored by, and what journal it should appear in.

Accordingly, the sponsorship of drug trials is inextricably linked to their design and outcomes. Out of 42 published articles of head-to-head comparisons of newer 
antipsychotic drugs, $33(78.6 \%)$ were funded by a pharmaceutical company and $90 \%$ reported outcomes in favor of the sponsoring company's drug (Heres et al., 2006). In different comparisons of the same drugs, this resulted in contradictory findings as "it appears that whichever company sponsors the trial produces the better antipsychotic" ( $\mathrm{p}$. 189). Two additional reviews of drug studies found odds ratios of 4.05 and 3.6, respectively, for a positive recommendation resulting from pharmaceutical industryfunded compared to non-industry-funded publications (Bekelman, Mphil, \& Gross, 2003; Lexchin, Bero, Djulbegovic, \& Clark, 2003). A recent analysis comparing outcomes of published clinical trials and FDA reviews of the same data for 12 antidepressant drugs discovered that $94 \%$ of the former showed positive outcomes, whereas only $51 \%$ of trial outcomes were positive according to the FDA analysis (E. Turner et al., 2008). These and other well-documented publication biases leave considerable doubt about the credibility of the major "scientific" source for drug information (Ioannidis \& Lau, 2001; Melander et al., 2003; Papanikolaou, Churchill, Wahlbeck, \& Ioannidis, 2004; Perlis et al., 2005). In an essay titled "Medical journals are an extension of the marketing arm of pharmaceutical companies," a former British Medical Journal editor suggested a remedy to this farreaching problem: medical journals should stop publishing clinical trials (Smith, 2005). In sum, FDA approval of a drug, which results from pre-clinical through Phase III testing, provides a "cognitive closure" in which clinicians and consumers accept the given "scientific" facts about the drug (Busfield, 2006). The drug testing and approval process may be ineffectual, though, because the pharmaceutical company makes the facts and selects the data to present. 
Limitations to scientific testing in psychopharmacology and the peculiar complexity of the industrial-regulatory process are not often noted once the official "badge of effectiveness and safety" is issued by the regulatory agency (p. 305).

Dissemination and credentialed practice of scientific drug knowledge.

In the offline world, scientific drug knowledge is typically disseminated to credentialed medical practitioners through published peer-reviewed journals (discussed in the previous section), pharmaceutical representatives or "detailers," medical education venues, and medical texts, particularly the Physicians Desk Reference (PDR).

Pharmaceutical companies are intimately involved with each of the above activities and products, with the effect of increasing the grey area between marketing and science.

\section{Pharmaceutical representatives, or "detailers."}

Marketing expenditures in the pharmaceutical industry have grown from $\$ 11.4$ billion in 1996 to $\$ 29.9$ billion in 2005, according to industry reporting and surveys (Donahue, Cevasco, \& Rosenthal, 2007). A recent independent source estimates a more realistic figure to be $\$ 57.5$ billion (Gagnon \& Lexchin, 2008). Physician and hospital detailing by pharmaceutical representatives is the largest expense, making up approximately $65 \%$ of marketing expenditures of top-selling psychotropic drugs. The job of the detailer is to provide "details" to the psychiatrist about the products they represent, and up to $90 \%$ of doctors report meeting with detailers (Lexchin, 1993) with an average frequency of 4 times per month (Wazana, 2000). At the same time, while many doctors indicate they place little value on detailers as a source of accurate and objective drug information, studies demonstrate strong evidence that detailing effectively influences doctors to prescribe the promoted drug. The significance of the detailer, however, is not only that 
such promotional practices are effective, but also that they represent an "extension of a hierarchically structured marketing apparatus - based on rationalized principles of management and market analysis - into social spaces previously thought to be occupied only by doctors and patients" (p. 285). Detailers track the effectiveness of their educational-promotional efforts by purchasing marketing maps that provide information on individual doctor's prescribing habits (Greene, 2004). Marketing maps may, for example, enable a detailer to target "stubborn" doctors with a different strategy, or to invite doctors prescribing in high volume to speak at a professional symposium. Pharmaceutical detailing represents one of the most targeted and well-funded promotional efforts aimed directly at individual clinicians who, under this scenario, may be considered another type of medical/health consumer.

Opinion leaders and medical-educational venues.

As an "experience good," the adoption of a new drug by psychiatrists is largely dependent on word-of-mouth and learning (Azoulay, 2002). This highlights the need for pharmaceutical companies to engage "opinion leaders" and to sponsor continuing medical education, professional symposiums, and scientific conferences. Opinion leaders are prominent experts in the field who receive honoraria and fees to speak at scientific or medical conferences, and to contribute to textbooks and journal articles (Relman \& Angell, 2002). Unlike pharmaceutical advertisements under FDA regulation, opinion leaders are able to diffuse to their colleagues the unapproved or novel uses of a drug. Pharmaceutical companies further provide the most support, in the form of funding and organization, of the above mentioned educational and professional events (Relman \& Angell, 2002). Some researchers have found bias in favor of the sponsoring company's 
drug in continuing medical education curricula, even when institutional CME guidelines were in place (Wazana, 2000). Additionally, psychiatrists often receive industry-funded meals, free samples, all-expenses-paid travel to attend pharmaceutical-sponsored educational events, and other gifts. Data from Vermont and Minnesota, two states that now require disclosure of drug company payments to doctors, indicate that psychiatrists earn more from pharmaceutical companies than any other medical specialty (Harris, 2007, June 27). Moreover, "psychiatrists who took the most money from makers of antipsychotic drugs tended to prescribe the drugs to children the most often" (para. 8). While doctors report that they are not influenced by pharmaceutical "education" and giftgiving, the evidence consistently indicates increased prescribing of sponsored drugs by doctors who attend such events or accept pharmaceutical gifts (Lexchin, 1993; Wazana, 2000).

Credentialed medical experts' practice of scientific drug knowledge.

Clinicians and psychiatrists, as licensed intermediaries between the drug and the end consumer, are provided access to scientifically produced drug knowledge through the above outlined channels (Lakoff, 2004). The knowledge actually utilized by these practitioners is typically more pragmatic than that produced by scientific methods, and reflects only the portion of scientific/medical knowledge that is relevant to the conditions likely to be encountered in routine clinical practice (Freund \& McGuire, 1999). This "recipe knowledge" may include such scientifically given facts as the recommended treatment for a condition or age group, side effects, and contraindications. Psychiatrists and clinicians may also rely on less "scientific" sources, such as popular media, the Internet, discussion with colleagues, and clinical experience (Phillips, Kanter, 
Bednarczyk, \& Tastad, 1991; Relman \& Angell, 2002; Woosley, 1994; Wyatt, 1991).

Patient requests may further impact practice with evidence demonstrating, for example, high physician compliance with specific or general drug requests $(53 \%$ and $76 \%$, respectively) to treat major depression (Kravitz et al., 2005). Of course, a variety of other factors may also help shape actual clinical practice, such as the need or desire of the clinician to satisfy the demands or anxieties of a patient, to quell an overwhelming workload, or to quickly terminate a difficult or time-consuming consultation (Weiss \& Fitzpatrick, 1997).

\section{Popular media and the Internet.}

The lay press may serve as a "filtering mechanism" for doctors and scientists to identify important findings from the vast amount of information found in medical journals (Phillips et al., 1991). However, on a daily basis health and medical reporters are "inundated with press kits, announcements, faxes...from drug companies and the public relations firms that represent them" ("Miracle drugs or media drugs?," 1992, March, p. 142). Popular media articles are commonly written with input from a pharmaceutical company's marketing firm as part of the public relations campaign to promote the drug (Healy, 2006b). Even media coverage of articles appearing in peer-reviewed journals have been found to frequently exaggerate findings, neglect harms, and fail to adequately identify study limitations or author conflicts of interest (Moynihan et al., 2000; Woloshin \& Schwartz, 2002).

While much of the research literature focuses on the Internet as a tool for consumers, psychiatrists and clinicians may also use the Internet to research clinical information, read articles from medical journals, and communicate with colleagues (Trickett, 2007, 
May 28). A 2002 survey of U.S. physicians found that most (70\%) report using three or fewer websites to regularly find medical information, with WebMD consistently topping the list of online sources (Von Knoop, Lovich, Silverstein, \& Tutty, 2003). Nearly all physicians $(96 \%)$ reported that information they found online impacted their knowledge of available treatments, including drugs. A slightly smaller majority (73\%) reported that the information impacted their prescription decisions.

\section{Clinical experience.}

In addition to official scientific drug knowledge funneled to clinicians through the previously discussed channels, clinical experience may be a primary source of knowledge utilized to make decisions about psychiatric diagnosis, and the appropriateness and effectiveness of a treatment (Charlin, Tardif, \& Boshuizen, 2000; Wyatt, 1991). This is especially so in selecting psychotropic drugs because the "difficulty in applying valid and sensitive measures of therapeutic effect" requires selection based on the individual's likely tolerability of adverse effects or knowledge of a previously favorable response to the drug (Brunton et al., 2006, p. 429). As already mentioned, the speech and expressions of the psychiatric patient are uniquely important in psychopharmacology, and are equally so in clinical practice with psychotropic drugs. Though because medical training and professional ideologies may effectively restrict problem-solving to the specialized tools of the profession (Nordin, 2000), it is likely that the clinician interprets the impact and meaning of drug effects differently than the individual experiencing it first-hand (McCubbin \& Cohen, 1996). For example, the psychiatrist may primarily seek congruence of the patient's story with prevailing diagnostic systems such as the DSM (Tucker, 1998), and some recognized scientific facts about the drug. Through taking the 
patient's treatment or "medication history," the clinician embarks on an interpretive process of meaning-making that may or may not remotely reflect the true nature and impact of the drug as experienced by the user (Cohen, 2003). This is partially empirically demonstrated by studies finding poor correlation between clinician-rated and patientrated psychopathology measures (Lasalvia, Ruggeri, \& Santolini, 2002). Clinician-rated psychopathology is also poorly correlated, while patient-rated psychopathology is moderately to highly correlated, with subjective quality of life.

Additionally, several studies have revealed insufficiencies in the process of relating expert drug knowledge to actual or potential drug users within the clinical consultation. In multiple surveys, tardive dyskinesia, a common serious adverse effect of certain antipsychotic drugs, was simply unmentioned by psychiatrists as a risk to patients. In a survey of patients taking antipsychotic medications, over half (59\%) reported that other treatment options had not been presented and $46 \%$ indicated that they had not received any information about potential adverse effects of the prescribed treatment (Gray, Rofail, Allen, \& Newey, 2005). In a survey of 223 patients receiving medications for a diagnosis of bipolar disorder, between $60 \%$ and $70 \%$ of respondents reported dissatisfaction with receiving too little information from their clinicians about adverse effects, what to do in case of experiencing an adverse effect, and whether the medication would affect sexual functioning (Bowskill, Clatworthy, Parham, Rank, \& Horne, 2007). After interviews with 51 patients starting antidepressant drug treatment, the most commonly reported unmet information need concerned adverse effects, followed by the role of treatment in recovery, expected length of treatment, and the risk of physical and psychological dependency on the drug (Garfield, Francis, \& Smith, 2004). Such 
omissions by psychiatrists and clinicians, whether intentional or not, violate the principle of informed consent and serve to undermine the credibility and reliability of clinical expertise.

Due to the distorting effects on scientific drug knowledge of extensive pharmaceutical company involvement in the production of drug fact-making and subsequent dissemination to practicing experts, it may be justifiably argued that clinicians are unequipped and unable to provide a "balanced" risk-benefit appraisal to drug consumers. Further, the esteemed status of the credentialed medical expert may be threatened if the specialized knowledge that distinguishes expert from non-expert loses credibility and relevance. Despite professional ethics and licensing requirements intended to instill trust and proclaim competence, many psychiatrists may themselves have only limited understanding of drugs' full effects.

\section{Consumer Drug Knowledge in the Off-line World}

In trying to come to a "valid" understanding of drugs' effects, the voice and speech of the patient has traditionally been muted or neglected in favor of a presumably more objective biomedical discourse (Hyden, 1997; Jacobs \& Cohen, 1999). With this, the depth and complexity of an individual's suffering and treatment are reduced or eliminated (Miller \& Crabtree, 2005). While it may be legitimately argued that the patient's "voice" is irrelevant to a scientific understanding of disease (defined in Western models as pathological alterations at cellular or molecular levels), the role of the patient's voice in psychiatric medicine might be profoundly different because there exist no accompanying physical signs or symptoms to aid a psychiatric diagnosis and treatment plan (except for substance-induced conditions and conditions, such as dementias, consequent to various 
physical diseases). Absent both strict disease determinations in psychiatry and patients' voices in psychiatric drug research, it is important to explore what power this biomedical discourse and professionally-mediated treatment reflect.

The drug consumer may consider information from a variety of sources to interpret and label symptoms and make treatment decisions. Common sources for the consumer include their doctor or psychiatrist, advice from non-medical helping professionals or non-professionals in their social network, scientific/medical texts such as the $P D R$ or the drug label, DTC advertising and popular media, Internet sites and discussion forums, personal and shared experiences, and personal beliefs about health, disease healing and medicine (Harmon, 2005; S. Williams \& Calnan, 1996). Consumers typically seek information that is congruent with the everyday experience of their problem (Kivits, 2004), which may or may not include strictly biomedical interpretations and categorizations. This carries potential for dissonance between the consumer's explanation and perceived needs, and psychiatric/medical explanations and cures.

A consumer's reliance on a clinician or psychiatrist for drug information may be caricatured on a continuum ranging from rejection of the value and/or validity of expert knowledge, to autonomy in gathering and digesting expert knowledge, to complete dependence on the expert for relaying knowledge and making associated treatment decisions (Fox \& Ward, 2006). Autonomous consumers generally accept medical explanations for their suffering and may carefully research treatment options prior to the medical encounter in order to offer their clinician specific treatment suggestions. Some may even lie about their symptoms to ensure they receive their desired drug (Harmon, 2005). Others may experiment with increasing or decreasing their already prescribed dose 
or trying different medications borrowed from a friend. Particularly among drug consumers under age 40 , the popular media have reported, "there's this increasingly widespread attitude that 'we are our own best pharmacist"' (para. 22) and that a medical degree "is useful, but not essential, and certainly not sufficient" (para. 6). As an aside, it may be hypothesized that consumers in the autonomous and perhaps rejection range would be more likely to contribute to consumer-generated online health and prescription drug sites than dependent consumers.

\section{Personal and shared experience.}

Any "expertise" or special knowledge a patient may be granted by professionals is rooted in experience (Caron-Flinterman, Broerse, \& Bunders, 2005). Consumers have first-hand knowledge of the impact of illness on their body and social life, and the benefits and pitfalls of tried treatments. The consumer is the only one who can decide the usefulness of the treatment for their life circumstances (Kazdin, 1999; Nordin, 2000). The notion of efficacy - a statistically significant superiority of the drug over placebo of paramount importance in psychopharmacology - is minimally relevant to clinical significance and the total drug experience.

Personal experience and the insight gained from others with similar experiences is often perceived and utilized by consumers as a relevant, useful, and trustworthy source for gaining knowledge and making treatment decisions (S. Williams \& Calnan, 1996). A recent survey found that experiencing an adverse effect, but not contact with a clinician, receipt of a medical diagnosis or admission to a hospital, was significantly related to increased awareness of a prescribed drug's adverse effects (Papanikolaou \& Ioannidis, 2003). Similarly, consumers may be warned, for example, about severe withdrawal 
effects of certain antidepressants from an experienced friend, but not from their prescribing physician who may be unaware of such an effect as it is often not part of the drug's official profile (Cohen, 2007). Inasmuch as drug effects are unpredictable for any one individual, the experience of others may serve as a trusted and "objective" - in terms of the direct testing of an "experience good" without apparent conflict of interest - source of information.

Some social scientists have strongly recommended viewing scientific and lay knowledges as necessary complements, suggesting that they are equal in value and contribution to the overall knowledge of a domain, though different in their content and epistemology (Goodare \& Lockwood, 1999; Popay \& Williams, 1996). Accordingly, either one or both may be wrong on occasion. This paper is further concerned with power relations and the politics of truth - that is, how scientific knowledge about drugs is legitimated (through associations of it as objective, reliable, and valid) while consumer knowledge is more often de-legitimated or made inferior (through associations of it as subjective, idiosyncratic, and naïve). This dynamic surrounding the production of drug knowledge is partly due to the construction of "medication" as an expert domain, and persists despite possible distortions in the objectivity, reliability, and validity of scientifically produced knowledge largely due to pharmaceutical companies' far-reaching involvement. The user-generated Internet introduces a new component to conventional power relations in drug knowledge production and dissemination. 


\section{Expert and Consumer Knowledge in the Online World}

The dynamics of knowledge on the web.

The original Internet was dubbed "the information superhighway" - a name which no longer seems suitable to describe its current dynamics and uses. Today's Internet variously called the programmable web, the user-generated web, or Web 2.0 - is about participation, collaboration, and communication (Tapscott \& Williams, 2006). The difference reflects more than just a shift in popular use of a technology, but rather suggests a greater cultural impact and a different way of thinking about knowledge. Dynamics of the user-generated Internet of interest for this paper include the evolving roles and expectations of consumers in the online realm, the potential of the Web to anticipate later acknowledged realities, and the competition for online space and attention.

The development of the user-generated web consisted of a bottom-up transformation of traditional practices in multiple market sectors (Tapscott \& Williams, 2006). The assumption of a passive consumer who quietly accepted a finished good (including information) or service was challenged by self-organized online consumer communities in media, journalism, software development, and other areas that fulfilled unmet needs in a grassroots way. By 2006, "the programmable Web eclipsed the static Web every time: Wikipedia beat Britannica; Blogger beat CNN; Epinions beat Consumer Reports... and craigslist beat Monster" (p. 38-39). These leaders among consumers have arguably ignited a shift in the relations of power to become something more like "prosumers" who participate in the creation or production of the good or service they are consuming (p. 125). Consumers more broadly appear to carry expectations for formerly 
static websites to provide opportunities to participate, collaborate, or interact with the site and/or with other users. Inevitably, such openness leads to multiple claims to knowledge and uncertainty as to claims' credibility and authenticity in an inherently non-transparent realm (credibility on the Internet is discussed in detail in following sections). Despite difficulties, the potential of Web 2.0 is ballooning as companies and research institutes in all sectors are grappling with innovative business, marketing, and research models to accommodate this cultural shift ("Let data speak to data," 2005; Tapscott \& Williams, 2006).

As a result of increasing consumer participation and interaction online, the Web may carry the potential to anticipate what only later is officially acknowledged as a reality (Rogers, 2003). In the case of psychotropic drugs, an actual or potential consumer on the Internet can find the official medical/professional/industry accounts of a drug's uses and effects, as well as unofficial accounts from consumers and "fringe" groups that may "enrich and complicate more official accounts" (p. 196). Insofar as the Web is a grassroots technology used by individuals to (often spontaneously) self-organize and "have a voice", the Web may be the "first to know" of novel or underground drug uses and professionally unrecognized desirable or adverse drug effects (p. 196). This is certainly the case with "brain shivers" - an adverse effect associated with antidepressant use and withdrawal, which was recognized by clinicians and researchers only after online discussion of the phenomena among consumers became too overwhelming to dismiss (Christmas, 2005). Clinical trial reports indicated some incidence of "dizziness," "vertigo," and "sensory disturbances" upon drug "discontinuation," but the experience of consumers was congruent with difficult-to-describe electrical shocks to the head and 
often a feeling of severe drunkenness - even after missing just one dose of certain antidepressants. The discrepancy, not only in terminology, but in depth of understanding resulted in individual doctors and pharmacovigilance centers dismissing, minimizing, or misclassifying consumers' complaints (Medawar, Herxheimer, Bell, \& Jofre, 2002).

Brain shivers continued to be defined and described so differently by experts and consumers that the phenomenon recognized by one appeared foreign to the other. Further, the medical community seemed reluctant to take seriously the "anecdotal" reports of consumers, despite the rapid growth of websites dedicated to discussing the issue. In this case, the Web was the "first to know" of brain shivers and could be described as anticipating a later acknowledged reality, as the medical community did eventually recognize this effect to be a "new" drug problem even without an understanding of its causal mechanisms. Of course, there are numerous examples in the off-line world of patients recognizing iatrogenic effects of an intervention before professionals and having their recognition contested or discounted (Popay \& Williams, 1996; S. Williams \& Calnan, 1996). The web as potentially anticipatory adds a new component to this existing power relation.

Finally, with unlimited virtual space on the Internet and the ease of publishing one's views online, the most valuable resource in the online realm may be attention (Dahlberg, 2005). Millions of web pages make it less likely that any one page will be noticed by an audience of considerable size. Search engines do not frequently reveal their algorithms for returning search term results, so it is not possible to definitively outline all of the most important elements in the determination of online attention. To tip the scales in one's favor may require maintaining an extensive network of inbound hyperlinks, 
reproduction of one's content on other sites, and costly pay-per-click advertising. Sites can also purchase such items as "prime time" and "sole supplier" status on search engines to minimize the chances that a competitor site will garner similar attention.

In the health domain, some medical researchers, practitioners, and regulatory authorities as high up as the World Health Organization are advocating for regulation of health websites using quality ratings and seals of approval (Eysenbach, Powell, Kuss, \& Sa, 2002; Fox, 2010). Such actions would presumably protect consumers from wrong or potentially dangerous information and, simultaneously, assert what gets counted as legitimate health information (Brown, 2002). These efforts have not been in vain as an unknown number of search engines "already exclude or marginalize health websites that don't meet recommended medical grading system requirements" (Lewis, 2006, p. 528), which may include consumer-led sites and other "unconventional" health resources.

However, the negotiation of space and attention is continually challenged by the Internet's openness. For example, the rise in consumer complaint forums has been met by companies "attempting to defend themselves by setting up anti-domains" before consumers have an opportunity to register them (Harrison-Walker, 2001, p. 398). Days prior to launching the site Priceline.com, the company purchased the domain Pricelinesucks.com and a variety of other spin-off names. The hugely popular video site YouTube allows individuals to post video clips which are searchable by other users using keywords attached to the video. A recent high-profile video featured a five minute segment from a former sales representative for Eli Lilly's antipsychotic drug Zyprexa, "who reveals what Lilly officials told him to say about the drug's side effects" (Thomaselli, 2007, p. 4). 
Pharmaceutical companies are in return posting positive and entertaining videos about their products on the site. Wikipedia is another prime example of the battle over virtual space (and knowledge in Wikipedia's case). A detailed look at Wikipedia follows in the Limitations section.

It is clear that the negotiation of space and attention on the Web is not as straightforward or transparent as one might easily believe, and certainly is not a haphazard or spontaneous occurrence. The above strategies to guarantee attention require extensive monetary or networking resources, favoring those corporations and organizations with pre-existing resources in the off-line world (Dahlberg, 2005). Quality ratings for health websites serve to reinforce traditional power relations in the production and dissemination of the respective knowledge. These dynamics reveal some of the inequalities in what is promoted as an inherently democratic medium supposedly governed by the free choice of users. The user-generated web is a contested terrain with competition for attention and claims to knowledge. The actual and potential altering of power relations around knowledge production has caused heated debate among researchers and social commentators about the evolving role of expertise in the age of the Internet.

\section{Credibility on the Internet.}

Users of the Internet may come across a variety of information on any particular health topic, and thus are no longer limited to receiving a pre-filtered presentation from a traditional gatekeeper such as a trusted doctor (Eysenbach, 2008). However, sources of information across all domains are non-transparent in the online realm. It is difficult to know what conflicts of interest and scientific or financial stakes are involved in the 
construction of expert sites, just as it is difficult to gauge the presence of unauthentic accounts contributed by persons with vested interests in consumer sites (Clarke et al., 2003). In parallel, an identity as an expert can be as easily misrepresented as an identity as a consumer. What this amounts to for Internet users is a shift in the importance of source credibility to a greater reliance on message credibility (Eysenbach, 2008). Rather than relying on any particular claim or assertion based on expertise, Internet users appear more often to cross-check bits and pieces of information across sites with less concern for whether the author is speaking from credentials or experience (David, 2007; Eysenbach, 2008; Sillence, Briggs, Harris, \& Fishwick, 2007).

This dynamic of the Internet causes some medical authorities to disapprovingly judge the Internet to be "an unruly, unregulated space marked by plurality of claims to knowledge and authority" (Lewis, 2006, p. 528). This perception has led to concern among many medical researchers and practitioners about the "quality" of information found online (Eysenbach et al., 2002; Ferguson, 2002). However, what may be needed is a new model for judging the credibility of these anonymous, distributed, co-constructed web sources that are becoming so popular and familiar among Internet users. One such is the "field-dependent model" in which credibility depends on the user accepting the principles and practices specific to the field or context within which the site operates (Warnick, 2007). For example, an independent media (Indymedia) site based on principles of open peer publishing, constructing alternative accounts, and the impossibility of objective reporting will not be judged credible using the same standards of mainstream journalism which is based on a very different set of principles. The fielddependent model explains that users "must look to his or her own assessments, based on 
what is said on the site, in its comments, its external links, and on other sites" in order to decide whether the content may be viewed as credible (Warnick, 2007, p. 62). Under this model and suitable to many medical domains in which criteria for providing "complete" and "accurate" information are necessarily problematic, credibility is a subjective concept arrived at with consideration of the user's prior experience, knowledge, and needs (Eysenbach, 2008). Consumer-constructed sites are not generally meant to provide scientific health information to readers, but rather exist for a variety of other purposes that may be judged "credible" in their own respective contexts. This model gives agency to Internet users and allows for different standards to be applied to the variety of web sources existing in multiple contexts. Of course, regulatory concerns regarding health content appear unjustified in the absence of equally vigorous attempts within the medical community to ensure the unbiased flow and content of "official" health information.

More broadly emerging is a re-evaluation of ways of producing what is judged to be legitimate knowledge in online communities. The emergent and community models illustrate how knowledge is differently produced within online groups. In the emergent model of a user-generated site, thousands of individual users each contribute a small part (like a review of a product) and out of this emerges a coherent body of work (Wales, 2005). Credibility on these sites is established through reputation mechanisms, such as the ranking system on Slashdot or the complex system of user reviews on Amazon. In Slashdot's ranking system of technology news stories, users gain reputation and influence by their invested participation in the community and their ability to meet the information needs of other users (David, 2007). 
The community model is characterized by a dedicated group of volunteers who work together to create and maintain the site's content (Wales, 2005). As in any organization or community, users get to know one another as part of their continued interactions and the collaborative process is thought to ensure the site's quality and credibility. The nonprofit, volunteer-led encyclopedic website Wikipedia is the most notorious example of the community model. A core group of about 524 users ( $0.7 \%$ of all Wikipedia users) do about half of the site's editing. Any Internet user may create or contribute to an entry anonymously or as a registered user, though only $18-21 \%$ of all edits are anonymous. The English-version Wikipedia has over 1 million entries and had reached top 50 website status by 2005, receiving more traffic than the New York Times (Tapscott \& Williams, 2006). The strengths of Wikipedia include its constant growth in the addition of new entries on obscure and niche topics, and its rapid and in-depth coverage of recent events. Wikipedia's openness also makes it vulnerable to inaccuracies, vandalism, and "edit wars" in which disagreeing users change one another's edits. However, despite the inevitability of errors in any encyclopedia, an obscenity randomly inserted in a Wikipedia article is removed in an average of 1.7 minutes. Disagreements and inaccuracies may be particularly a problem for entries in philosophy, politics, and culture, but for science topics Wikipedia has recently been found to be about as accurate as The Encyclopedia Britannica (Carnevale, 2006). In a study of exposure of user-generated sites in popular search engine returns for health-related searches, Wikipedia appeared on the first page of Google and Yahoo in 63\% of searches, making it the most frequently cited usergenerated resource ("Diving deeper into online health search," 2007). 
Thus, "Wikipedia, like the larger open system of the Internet, is charting new territory in which widespread use is not contingent on widespread trust, at least on the terms established by earlier expert systems" (David, 2007, p. 185).

The credibility of the user-generated web and of individual contributions within specific websites is an unresolved and evolving issue. Despite their lack of credibility in certain quarters, user-generated sites are highly trafficked and carry validity for their constituents and users. Revisions to concepts of truth, credibility, legitimacy, and expertise brought on by the Internet are at the heart of discussion and debate among Internet researchers in a variety of fields (David, 2007; Keen, 2007; Warnick, 2007). As a beginning attempt to add to this discussion in the area of psychotropic drugs, the present research sought to compare the information found on expert and consumer online sources. This is to distinguish the aim of the research from one that seeks mainly to determine the truth value of specific claims.

\section{Consumer drug knowledge and the Internet.}

Consumers may use the Internet to supplement, verify, translate, or replace information from other sources (Eng \& Beauchamp, 2005). The almost unlimited information available on the Internet creates the opportunity to find personalized and individualized information, as consumers can keep searching until they find information that resonates with their experience and beliefs. A 2002 survey of 10,000 U.S. patients found that most patients (75\%) report visiting 2 to 5 websites regularly to find health information (Von Knoop et al., 2003). Similar to results from physician respondents, patients consistently report WebMD as a top online source. WebMD is constructed and monitored by medical professionals and contains health information presented as 
scientifically legitimated. It is intended for an audience of other experts and the lay public. It is unknown whether the reported popularity of WebMD is due to its relevance to and fulfillment of information needs, or to its success in negotiating online space through search engine placement and advertisements.

Second, consumers may use the Internet to share their experiences, give advice and recommendations, or act as a resource linking individuals to other relevant sources (Hardey, 2002). This use of the Internet transforms consumers into producers of health information (Hardey, 2001). Personal web pages describing illness, for example, may be considered "a new genre" of the illness narrative, in which the story is unmediated by a health professional or researcher, receives guaranteed instant publication to a global audience, and is dynamic in nature in that the story can be continually updated (p. 395). Information-, advice-, and resource-sharing are similarly accomplished in online community groups, discussion forums, and chat rooms, with a range of discourses potentially represented (Fox \& Ward, 2006). In a consumer-led discussion forum called CrazyBoards, "fluency in the language of psychopharmacology is taken for granted. Dozens of drugs are referred to in passing by both brand name and generic, and no one is reticent about suggesting medications and dosage levels" (Harmon, 2005) (para. 37). In this case, users approximate the "autonomous" consumer discussed previously, as the doctor appears necessary primarily to purchase the desired drug rather than for information-gathering and decision-making.

Finally, the frontier of Internet uses by consumers may include creation of a consumer research community in which consumers across the country offer ongoing data about their symptoms and treatments in order to create a large database comparable to, and 
more timely, accessible, and relevant than, data from clinical trial research. One website, PatientsLikeMe.com, has been exploring this frontier since March 2006 with consumer research communities for Lou Gehrig's disease, multiple sclerosis, HIV, and, as of early 2008, mood disorders (Goetz, 2008, March 23). Participants on the site can provide information as often as they wish on their symptoms, symptom severity, treatments, and dosages, and the website's software automatically converts the information into charts and graphs for ongoing monitoring. These quantitative measures are supplemented with discussion forums that provide participants opportunity for elaboration. Further, by filling out a personal profile, the site instantly matches up a newcomer with other participants who share similar symptom and/or treatment profiles. The creator of the site, the brother of a person who died from Lou Gehrig's disease, envisions that it "might complement large-scale and long-term clinical research by conducting observational research 'on the fly"' (para. 52) and supports this by adding that data "are present not just in laboratories or universities or proverbial halls of science but in everyday life" (para. 54). Such research came to fruit in November 2007 when a small group of the Lou Gehrig's disease community chose to seek lithium treatment from their doctors on the basis of an unpublished study in Italy that showed promising results. Each member of the group uses the site to share and track treatment effects with the intent to expeditiously verify or cast doubt on the scientific research results. With this and future possible drug "trials," the creators and users of the site perceive the site's potential to transform health research:

most health-care data is inaccessible due to privacy regulations or proprietary tactics... When you and thousands like you share your data, 
you open up the health-care system... We believe that the Internet can democratize patient data and accelerate research like never before. (para.

PatientsLikeMe.com has received attention in the New York Times and numerous health blogs as a potential disrupter to the health care industry and medical research (Goetz, 2008, March 23; Schonfeld \& Morrison, 2007). They also published a paper about Lou Gehrig's disease in the European Journal of Neurology and received their first scientific award for a poster presentation in 2007 at the British Neuropsychiatry Association (Wicks, 2008; D. Williams, 2007). When proactive consumers take it upon themselves to create and disseminate the knowledge they feel a need for and even form their own drug trials, it may arguably represent a contestation of consumer exclusion from conventional processes of knowledge production.

\section{Expert drug knowledge and the Internet.}

Just as there has been an explosion in consumer-constructed health websites, there has been an equal explosion of expert-constructed sites. These include international and governmental health authorities, healthcare hospitals, academic institutions and insurers, medical research organizations and databases, professional associations, pharmaceutical companies, non-profit organizations formed around a particular psychiatric diagnosis, individual and group medical expert web pages, and many more (Parr, 2002). Such expert sources typically aim to interpret and/or disseminate scientifically legitimated medical knowledge about psychiatric diagnosis and treatment. The Internet, in contrast to all other mediums, provides a means to accomplish this in a highly accessible and up-to-date manner. At the same time, the dynamic linking of web pages means that the producers of 
messages have decreased power over the placement, timing, and context within which their message is presented (Weare \& Lin, 2000). Thus, the Internet "is arguably facilitative of a different kind of knowledge base about health and illness (it is not just a new and different medium, but helps to alter the nature of the knowledge accumulated)" (p. 78). While interesting to note, it is beyond the scope of this review and of the proposed research to explore how medical knowledge may actually be transformed by this new method of transmission.

Pharmaceutical companies, like companies in all sectors, are finding innovative ways to communicate with consumers online and maintain a strong market presence. Direct-to-consumer prescription drug websites have been found in multiple analyses to present incomplete and limited drug information, especially concerning drug risks and harms (Davis, Cross, \& Crowley, 2007; Hicks, Wogalter, \& Vigilante, 2005; Macias \& Lewis, 2003). Using a sample of 44 drugs, Davis and colleagues (2007) compared risk statements on the manufacturers' websites to statements on the FDA label. Websites promoting drugs with only 1 adverse effect occurring at a rate greater than $10 \%$ generally reported this effect $(90.9 \%)$. Only $15.3 \%$ of websites promoting drugs with 4 or more adverse effects occurring at this rate reported all of these effects. The greater the number of adverse effects, the less likely the consumer would learn of them on the manufacturer's website. This finding is particularly notable given the unlimited virtual space available to present comprehensive information. Hicks and colleagues (2005) examined the structure of 20 randomly selected drug manufacturer websites to determine the relative accessibility of information pertaining to benefits and risks. Risk information was found to be located deeper into the website structure and more difficult to access 
than information about benefits as measured by the amount of scrolling and number of clicks required for viewing.

However, DTC websites are only the tip of the iceberg in online communication. Pharmaceutical companies looking for new opportunities to influence consumer behavior are keenly aware of the power of consumer forums to build trust and offer support (O'Neill, 2007). A DTCA strategy termed "patient-centric marketing" recognizes that "the most effective method for true health engagement includes online patient communities" (p. 13). This involves recruiting "brand advocates" to build an "online community of like-minded consumers" who can create content that is "clear, jargon-free, and provided by the 'citizen' for the emotional and personal credibility" (p. 16). It is not known whether a "consumer" site sponsored by a pharmaceutical company or a pharmaceutical representative visiting a genuine consumer site would acknowledge the sponsorship or presence.

Another innovation draws on the influence of "respected physicians" to suggest specific health websites to their patients (Von Knoop et al., 2003). A pharmaceutical company may offer "prepackaged content for physicians' personal websites," which ensures that "doctors and patients alike find consistent messages... about the appropriateness of a particular treatment option" and that "information about a company's product is perceived [by the patient] as objective and credible" (p. 32). Finally, similar to pharmaceutical company financial sponsorship of medical journals and medical educational venues, one study found that $58 \%$ of the top 50 websites retrieved from Google and Yahoo searches of the term "schizophrenia" were funded by drug companies (Read, 2008). Most frequently, these were pharmaceutical company sites, sites 
that represented or served families of persons with schizophrenia, commercial sites with product for sale, and non-governmental organizations. Pharmaceutical company funded sites were significantly more likely to espouse a biomedical cause of schizophrenia and focus on psychotropic drug treatment.

The participation, collaboration, and interaction that characterize the usergenerated web are obviously equally applicable to consumers and corporate stakeholders. The Internet allows any individual with online access to produce or consume information without a professional intermediary, though, in many instances it appears that the immense resources and allies of pharmaceutical companies still tip the scales in their favor.

\section{Previous Forms of Internet Research}

An unknown number of websites allow consumers to rank and review consumer products and services, such as digital cameras, software, airlines, and now prescription drugs. Research on consumer review sites and online community groups related to mental health issues and drug use is limited in number and scope. Many qualitative studies into discussion groups for depression describe benefits of community group participation, such as social support and coping, or describe help-seeking behaviors in the online context (Powell, McCarthy, \& Eysenbach, 2003). Content analysis has been used by some researchers to examine the completeness or balance of drug information found on pharmaceutical company websites (Davis et al., 2007; Macias \& Lewis, 2003; Waack, Ernst, \& Graber, 2004). Several other studies have used content analysis, grounded theory approaches, or a mix of the two to describe characteristics and themes of patient/consumer discussion groups. In one such mixed approach, the researchers created 
codes for their pre-identified conceptual concerns in an electronic support group for persons with fibromyalgia, and created additional codes for emerging themes identified during readings (Barker, 2008). In fibromyalgia (Barker, 2008) and multiple sclerosis (Parr, 2002) online patient forums, researchers concluded the groups at various times (and sometimes simultaneously) contested and subverted expert medical knowledge, as well as reinforced and expanded the scope of expert medical knowledge. At least two additional studies have used solely a grounded theory approach to compare themes in online discussion board and narrative content between males and females with breast or prostate cancer (Gooden \& Winefield, 2007) and posthemorrhagic stroke (Stone, 2007). These studies were interested in differential informational and supportive needs for men and women, and the potentially supportive role of the online group, after diagnosis and treatment.

Few studies have attempted to explore consumer sites for their potential contribution to the overall knowledge of a topic, such as psychotropic drugs. One study retrospectively analyzed 1 year of discussion on an online forum for individuals with Parkinson's disease for information pertaining to adverse effects of prescribed drugs (Schroder, Zollner, \& Schaefer, 2007). Using a structured classification system for adverse drug effects to analyze the data, the researchers identified 153 adverse drug reactions on the forum, including a high rate of dermatological problems consistently described by participants as being "severe and stressful" (p. 1163). In the case of one antiparkinsonian drug, the incidence of skin problems in clinical trials was $0.8 \%$ compared to a $23 \%$ rate of mention in discussion forum entries. In contrast, cardiovascular adverse events, which were identified in clinical trials as a primary risk, 
were rarely discussed in the forum. It thus appeared that consumers online were more likely to discuss effects with the most burden or most obvious manifestation.

Finally, there are a dearth of studies that go beyond the boundaries of conventional methodologies to explore power and discourse in the online realm. Moore and Clarke (2001) used a critical interpretive approach to compare the conventions and heterogeneity of medical and popular online representations (visual and textual) of human genitalia. Their analysis included biomedical images for medical students, images found in popular newsgroups about human sexuality, and pornography. It appeared that traditional patterns of anatomical representation abound online, offering a narrow, technical, and singular view of genital anatomies. Pornographic sites provided the most diversity in representing various body types and ages, though in this genre each variation was fetishized for a consumerist purpose.

Rogers and colleagues are pioneers in mapping debate and discourse on the Internet, as they have done for issues of climate change (Rogers \& Marres, 2000) and genetically modified foods (Rogers \& Zelman, 2002). They accomplish this through mapping hyperlinks to determine the relevant players in an issue debate and to describe the concentrations and patterns of hyperlinks within and between various types of sites (i.e., .com, .org., .gov). They further identify and trace the recurrence of key phrases across sites in an issue debate. In the case of the climate change debate, the presence of a statement by the United Nations appeared to have particularly shaped the debate. Actors in the debate used the officially recognized, scientifically-backed statement to affirm, reinforce, contest, or otherwise position their own stance. 
In studying the web as an anticipatory medium, Rogers (2004) gave the drug Viagra a "more honest identity" by capturing and exposing the range of uses, experiences, and arguments about the drug from a variety of actors (p. 24). He used groups of Internet "surfer-experts" to answer a basic question: "What is Viagra, and whom is it for?" (Rogers, 2003, p. 198). The surfer-experts were instructed to compile lists of Viagra uses found in accounts of the drug from any source, and were given free reign to search the Internet using their personally preferred methods. Lists were compared among the surfers resulting in the conclusion that Viagra "has become a lifestyle drug for men in their 30s and 40s, to be obtained from virtual doctors, having had referrals from 'death by Viagra' search engine queries or from banner ads on porn or racy sites" (p. 204). The exploration of unofficial online accounts of the drug led the researchers to further conclude that "Viagra leads a richer, more youthful and experimental life than it is granted by doctors, the medical industry and by the manufacturer - all of whom retain Viagra as a prescription drug for a patient with a medical ailment" (p. 209).

The present research built on the distinct aims and methods of earlier projects by describing the content and themes of expert and consumer sites starting from a critical conceptual approach and using grounded theory methods within a mixed-methods research strategy.

\section{Grounded Theory}


Grounded theory was originally developed by Glaser and Strauss (1967) for the purpose of generating theory grounded in empirical data, as opposed to testing hypotheses based on abstract theorizing. Theory generation is accomplished by using a number of analytic tools. The most popular are coding, memo-writing, theoretical sampling, and the constant comparative method. The grounded theory research process is an iterative process of collecting and analyzing data, with the expectation that codes and concepts derived remain provisional as the research progresses. This method most closely resembles an inductive process, but arguably requires both inductive and deductive inquiry (Berg, 2000). The researcher is formulating hypotheses about appropriate concepts and connections between them, while continuing to test these hypotheses (using the constant comparative method, for example) and generate new ones with additional data collection and analysis.

Since its origin, the above basic grounded theory guidelines have been used as part of a variety of research strategies and for multiple aims other than building theory (Corbin \& Strauss, 2008). A number of methodologists recognize that grounded theory guidelines are best viewed as a set of principles and practices for conducting qualitative data analysis, rather than a set of methodological rules or requirements (Charmaz, 2006). Its original developers, Glaser and Strauss, too, eventually disagreed on the appropriate methods for conducting grounded theory research and continued developing the method independently of one another (Denscombe, 2007). Glaser believed the researcher should maintain a distance from the data and allow meaning to emerge by a neutral examination of manifest content. Strauss adopted a more interpretive approach claiming that the role of the researcher was to find meaning in the data, which usually required looking beyond 
superficialities. More recently, several scholars have provided constructionist and postmodern adaptations of grounded theory (Charmaz, 2006; Clarke, 2005). The present research drew from elements of recent versions of grounded theory, including the work of Corbin and Strauss (2008), Charmaz (2006), and Clarke (2005).

In their most recent edition of Basics of Qualitative Research, Corbin and Strauss (2008) describe grounded theory as a general approach to research that is appropriate not only for theory development as originally formulated, but also for superficial description or in-depth analysis. In-depth analysis "is more likely to generate new knowledge and deeper understandings because it tends to go beyond what everyone already knows" (p. 51). The present research aimed for an in-depth analysis, which included a presentation of reported medication effects and generated themes/categories. In keeping with the social constructionist framework, categories/themes were not assumed to emerge from the material, as "rather than discovering order within the data, we create an explication, organization, and presentation of the data" (Charmaz, 2006, p. 140).

\section{Summary}

Pharmaceutical industry influence over the production and dissemination of scientific drug knowledge on which clinicians, other professionals, and consumers may rely is ubiquitous and inescapable. The effect of such influence is the extensive blurring of marketing and science, and an emerging critical questioning of the validity and integrity of the "scientific" evidence base for psychotropic drugs. In parallel, the Internet has provided an opportunity to review drug information from thousands of consumers without professional mediation. Most research positions expert health or drug knowledge as the standard, authoritative source to which other forms and sources of knowledge are 
compared for their accuracy, completeness, validity, and so on. It is unknown how consumer-constructed and expert-constructed web sources for psychotropic drugs may compare if neither form of knowledge was a priori privileged as a standard.

\section{Conceptual Approach}

The central issue of the proposed research concerns the construction of expertise in the age of the Internet. The research asks: Has the Internet rendered expertise in the area of psychotropic drugs obsolete? The validity, credibility, and integrity of scientific/medical knowledge in the area of psychotropic drugs have become highly contested in recent years, as extensively explored in the review of literature. The Internet is 1) an evolving communication medium, 2) a network of computers and people, 3) a socially constructed virtual reality, and 4) a potentially transforming component to traditional power relations in knowledge production and dissemination (Markham, 2004). The push-and-pull dynamics of traditional and presently evolving power relations, illustrated in Figure 2.1, leads to an unspecified situation for the production and dissemination of psychotropic drug knowledge in the Internet age.

Social constructionist (Berger \& Luckmann, 1967) and critical perspectives (Foucault, 1980) frame this investigation into the production of psychotropic drug knowledge on the Internet. This research takes the position that there is no pre-constituted objective social world waiting for specific procedures to unveil and articulate. Working from Foucault's concept of power/knowledge, "truth" is not so much discovered as it is produced according to historically situated conditions that make up the politics of truth that is, who is authorized to speak, what objects are worthy of inquiry, and the techniques and procedures for acquiring truth and enabling one to distinguish between true and false 
statements (Rawlinson, 1987). However, to say that truth is constructed, or that drug effects are constructed, may be a misnomer as it implies a falsely clear distinction between the "objective" existence of the physical object, and its "constructed" existence of representations and meanings (Mitchell, 2002). It may be more precise to say these things are "made," or according to Foucault "produced," out of processes that are at the same time material and cultural, and as much real as abstract. Teasing apart the "real" object, for example the physical drug, from its personal, social, cultural, political, and economic meanings and uses is arguably a fruitless task because all of these elements interact to produce what a person experiences as or what scientists make to be "psychotropic drug."

This perspective also has a number of implications for the online textual material used for analysis in this research. "Text" here specifies any written language, images, symbols, or other that "means something to someone, it is produced by someone to have meanings for someone else" (Krippendorff, 2004) (p. 19). First, a text's message or meaning is not something objective to be found or identified by the researcher, but rather is subject to an interpretation process according to the context developed by the researcher (Krippendorff, 2004). Researchers working within different disciplines, such as economics, psychology, or political science, will interpret the same text from different perspectives and within different contexts. None of the resulting analyses would be more "right" than the other, just as none would represent "the" message or meaning inscribed in the text.

Second, a social constructionist perspective rejects the traditional sender-receiver model of communication in favor of the consideration of intertextuality, meaning that 
text is always related to larger discourses and is often understood in reference to presumed knowledge of other texts (Titscher, Meyer, Wodak, \& Vetter, 2000). For example, a text may explicitly or implicitly articulate a position on the role of patients in healthcare decision-making within a biomedical, political, or consumer activist discourse. As humans are "routinely both producing and awash in seas of discourses," so are texts, as products of human thought and action, embedded in these discourses (Clarke, 2005, p. 145). One text may be connected with multiple and various, sometimes conflicting, discourses. Also, ascribing meaning to a text often depends on the reader's presumed knowledge of other texts, which may include general everyday knowledge or experience, publicized current events, or an understanding of humor or satire, among others (Warnick, 2007). Consequentially, "Because of intertextuality, there can in principle be no objective beginning and no clear end, since every discourse is bound up with many others and can only be understood on the basis of others" (Titscher et al., 2000, p. 26).

Third, text content speaks to something other than itself. For example, words and images may inform, evoke a feeling, encourage action or behavioral change, and much more. This implies that the researcher must look beyond the physicality of the text to consider how the text is used and what and how it encourages. At the same time, meaning making on the Internet "is an emergent process that commences when the user first encounters the site and then develops his or her understanding based on signs, pathways, forms of expression, and representations encountered on the site" (Warnick, 2007, p.

104). Moreover, users can create entirely different texts in their use of hyperlinks so that one user's individuated pathway is nearly non-replicable. The fragmented and hyperlinked nature of the Internet gives the creator of an online text little control over the 
context within which the text will be discovered or how the text may be interpreted in light of that context. Yet, authors of online text may still be deliberate and strategic in the use of hyperlinks to position the site within an issue network (the entire network of linked organizations and sites around a particular issue, such as climate change or childhood autism) (Rogers \& Zelman, 2002). To hyperlink to another organization makes the players and positions of the linked organization relevant to the overall discussion or debate, and "In this way, the act of not linking, non-reciprocal linking, or un-linking similarly reveals a politics of association" (italics in original; p. 3). In sum, different readers will navigate to and from the same site differently, making it difficult or impossible to represent a "typical" user reading of either expert- or consumer-constructed drug accounts.

Finally, discourse in the proposed research is defined as "communication of any kind around/about/on a particular socially or culturally recognizable theme," which may entail analysis of language, images, symbols, and nonhuman objects (Allan, 2006, p. 148). According to Foucault, discourse creates "conditions of existence" for what is possible and not possible to say, just as power/knowledge creates "conditions of possibility" for what is acceptable and not acceptable to say as true (Peterson \& Bunton, 1997). Individuals' language to describe personal problems, health, illness, or treatment, then, is enabled and constrained by culturally available explanations proliferated through discourse. Discourse also partly constitutes the object it describes through the use of discursive strategies (Rabinow, 1984). For example, a discursive strategy utilized in biomedical discourse may be to construct a subject - "patients" - on an axis of disempowered/empowered with an increase in empowerment contingent on gaining 
certain medical knowledge. The typically taken-for-granted functions and strategies of discourse must be continually considered and assessed in research comparatively analyzing consumer and expert descriptions of a treatment, or else the researcher carries the risk of again reinforcing traditional power relations and norms of popular discourse. Research Questions

The main question of the proposed research is: Does the accumulated consumer or layperson knowledge that the Internet allows dissolve the traditional boundary between expert and consumer/lay knowledge?

The research specifically sought to examine the content of drug information in consumer-constructed and expert-constructed web sources. This was accomplished by using a grounded theory approach resting on critical and constructivist frameworks to analyze the content of five websites containing drug information for two popularly prescribed psychotropic drugs from different drug classes. Also, as a practical consideration, the research asked, how does each source help and hinder treatment decision-making - both for clinicians and for actual and potential consumers? 
Figure 2.1 Traditional and Evolving Dynamics in the Production of Psychotropic Drug Knowledge

Dynamics of traditional power
relations that maintain the authority
of expertise in producing
psychotropic drug knowledge

Reliance on gatekeeper for access to (pre-filtered) knowledge

\section{Disqualification/dismissal of lay/ consumer "voice" or prospect of a consumer knowledge}

Contestations to expert knowledge are hidden

Scientific procedures are the only way to produce valid knowledge

\section{Medicalization and consumerism disperse and create consumer demand for expertise}

Pharmaceutical companies blur science and marketing, degrading the integrity and validity of expert knowledge
Evolving dynamics introduced with the user-generated Internet

Reduced need for gatekeeper for access to knowledge

Expectations of participation, interaction, and collaboration with consumers

Contestations of and multiple claims to knowledge have a direct outlet

Broadened conceptions of what may be deemed legitimate and credible knowledge

Responsibility for health and lifestyle choices is easier to maintain in daily lives with greater access to expert discourse

Pharmaceutical companies blur authenticity of online "consumer" accounts

Successful negotiation of space and attention may require preexisting resources and networks of relationships
Expertise in the age of the Internet in pro ducing psychotropic drug knowledge 


\section{Chapter 3: Methodology}

Research Strategy

A grounded theory approach was used as part of a mixed-methods research strategy for an in-depth textual analysis. The grounded theory approach was defined in a broad sense: "a research strategy that involves working with the concepts and categories of the research subjects" (Dyson \& Brown, 2006, p. 190). A primary competing research strategy for textual materials is content analysis, in which analysis is guided by prestructured expectations. Specific to textual analyses of drug effects, researchers utilizing a content analytic approach might use a hierarchical classification system used in medical research, such as the MEDRA dictionary or a drug-specific effects checklist, to guide coding. However, these classification systems are typically designed for coding adverse drug effects, and therefore already reflect a particular perspective related to drug use. That is, the dominant medical perspective that classifies psychotropic drug effects into main/therapeutic effects and side/adverse effects is built into these classification systems. As discussed earlier, however, there is reason to believe that users do not experience effects this way and that the main effect/adverse effect division is often arbitrary and changeable (Moncrieff \& Cohen, 2009).

A significant component of the present research, and the fundamental point of departure between the present research and previous work in this area, was the consideration of power related to knowledge. Most previous analyses of consumer treatment accounts have included only thematic analyses or have imposed medical classification systems or standards on the data prior to analysis. For this research, it was presumed that consumer perspectives and experiences were best represented by 
maintaining the concepts and categories that consumers provided, rather than classifying them into some form of a dominant medical framework of drug effects. The grounded theory approach was selected for the present research because it helped extinguish some imbalances of power (if they exist) related to the construction of labels and categories by not privileging either source (expert or consumer) as a standard for quality or accuracy.

The grounded theory approach is arguably one of the more interpretive qualitative research methods, as there is little pre-established structure to guide coding. It is difficult for other researchers to replicate a grounded theory study, which may be the major problem and limitation of this approach. The present study attempted to address this limitation through a couple of modifications. While still interpretive and flexible in terms of not applying an outside pre-determined template of codes and meaning on the data, the codebook was "fixed" at certain points in the process to calculate inter-coder agreement indices. Many codebook revisions were decided by agreement between two coders, rather than by the interpretation of a single coder. In these ways, the coding process was "standardized" more than would be expected in a strictly qualitative, grounded theory study. Further, most analyses included some combination of frequency tables, chi-square tests for independence of categories, and descriptive excerpts from the text.

In sum, this research followed a unique mixed-methods strategy based on a grounded theory approach to analyzing text. The influence of power on information was a central component of this research, and was best considered using a grounded theory approach. Other, more structured research strategies to analyze this textual data may have produced a different set of results, particularly in terms of the naming of drug effects and in their ultimate classification. For example, this study ended with no clear division 
between many conventionally considered therapeutic and adverse effects, a result which likely would not have been possible had the researcher started with a classification system structured to record "adverse drug effects." The major leverage this grounded theory approach offered over other strategies, then, was a critical analysis (in terms of examining an issue from outside the mainstream or dominant framework) of data that well represented (in terms of groundedness) drug experiences.

\section{The Sample}

\section{Website typologies and inclusion criteria.}

Five exemplar websites were sampled for in-depth analysis according to their status as expert-constructed (two sites) or consumer-constructed (three sites) sources with the goal of representing typologies. Representing a variety of websites (as opposed to, for example, a greater variety of drugs) was important to this study because it related to practical concerns of consumers who may question how drug information is similar or different across sites. Table 3.1 summarizes these typologies. Expert-constructed websites were categorized as professional health and consumer-centric, and one site representing each category was selected for analysis. Professional health sites are created and monitored by professionals in the medical or health field, typically involving medical journalists who gather and write content, and medical doctors who serve as consultants and oversee content. Content on professional health sites is promoted as reflecting recognized standards of scientific and medical excellence. For the purposes of the present research, then, the key criterion marking a website as "professional health" is the oversight of content by a team of medical doctors. Professional health sites are intended for both lay and professional audiences, and may include a component for discussion 
among the site's users. Consumer-centric sites are similar in all ways except for the latter.

As opposed to having an added component for user interaction, consumer-centric sites attempt to infuse principles of Web 2.0 by specifically designing the site to maximize participation and interaction of users.

Table 3.1

\section{Description of Website Typologies}

\begin{tabular}{|c|c|c|c|}
\hline & Typology & Description of Typology & Selected Site \\
\hline \multirow{2}{*}{ 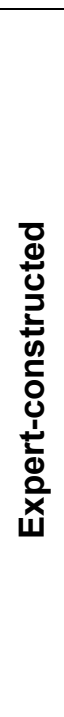 } & $\begin{array}{l}\text { Professional } \\
\text { health }\end{array}$ & $\begin{array}{l}\text { - created and monitored by medical or } \\
\text { health professionals } \\
\text { - reflect recognized standards of } \\
\text { scientific/medical excellence } \\
\text { - intended for both lay and professional } \\
\text { audiences } \\
\text { - may include a component for discussion } \\
\text { among the site's users }\end{array}$ & www.webmd.com \\
\hline & $\begin{array}{l}\text { Consumer- } \\
\text { centric }\end{array}$ & $\begin{array}{l}\text { - created and monitored by medical or } \\
\text { health professionals } \\
\text { - reflect recognized standards of } \\
\text { scientific/medical excellence } \\
\text { - intended for both lay and professional } \\
\text { audiences } \\
\text { - specifically designed to maximize } \\
\text { participation and interaction of users }\end{array}$ & www.revolutionhealth.com \\
\hline \multirow{3}{*}{ 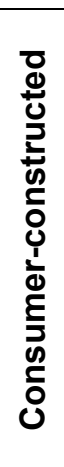 } & $\begin{array}{l}\text { Rigid structure, } \\
\text { narrowly } \\
\text { defined function }\end{array}$ & $\begin{array}{l}\text { - pre-defined fields for user input } \\
\text { - limited ability for extended conversation }\end{array}$ & www.askapatient.com \\
\hline & $\begin{array}{l}\text { Loose structure, } \\
\text { defined but fluid } \\
\text { function }\end{array}$ & $\begin{array}{l}\text { - no or few pre-defined fields for user input } \\
\text { - conversation is structured around } \\
\text { medication classes/brands }\end{array}$ & www.crazymeds.us \\
\hline & $\begin{array}{l}\text { No structure, } \\
\text { vaguely defined } \\
\text { function }\end{array}$ & $\begin{array}{l}\text { - no pre-defined fields for user input } \\
\text { - users construct structure and function by } \\
\text { adding conversations/threads }\end{array}$ & www.theicarusproject.net \\
\hline
\end{tabular}


Inclusion criteria for expert-constructed websites were: 1) the site was operated by a non-governmental group or organization, and 2) there was no evidence that the selected websites were owned by the same company or that they share professional contributors. The latter criterion was stipulated because many popular news and health sites exist as networks in which medical and other professionals who write or oversee content do so for the entire network of sites. Analyzing two expert-constructed sites that were designed by the same team of professionals would have been redundant in this research. Criteria for the selected websites were verified by examining pages within the "about us" (or similar) section. If the site was owned, for example, by a private or public corporation, then criterion 1 was met. For criterion 2, the staff, editorial team, expert reviewers, and management boards as listed on each website was examined by the principal investigator.

In contrast, consumer-constructed sites pertaining to psychotropic drugs are more heterogeneous in terms of the structure and function of the site - from 1) rigid structure and a narrowly defined function, to 2) loose structure and a defined, but fluid function, to 3) no structure and vaguely defined functions. An exemplar consumer-constructed site was selected to reflect each point along the continuum of structure and function. Multiple and varied discourses surrounding drug treatment were anticipated to naturally appear on this continuum due to the lack of a unitary "voice" among consumer discourses.

Inclusion criteria for consumer-constructed websites were: 1) the site content was viewable without requiring registration or membership conditioned on moderator approval, and 2) the site had a minimum of 200 contributions to an identifiable section or discussion about psychotropic drugs. The latter criterion was included because a 
preliminary search for consumer sites on the Internet revealed many sites with as few as three contributions to a discussion, which would not have furthered the aims of this research. Requiring a minimum of 200 contributions narrowed the consumer-constructed sample to those most likely to enrich the analysis. This also introduced a selectivity bias that favored larger websites. Larger website size may reflect greater fulfillment of the needs of site users, greater resources or marketing ability to garner attention, or something more random, such as being mentioned by a popular media outlet. Thus, small websites with few contributors were excluded from selection in this study.

\section{Description and limitations of search strategies.}

This research used a combination of search engine and collector site searches, and field immersion to sample websites that best represented the outlined typologies. Given the range of website typologies under consideration, it was difficult to apply a standard search and selection criteria for all 5 sites. Expert sites typically appear at the top of most search engine results, but may be better selected for the purposes of this research according to their "quality" rank by highly regarded collector sites. Consumer sites may appear in the first few pages of search engine results (after the first few pages, search returns become barely related to the search terms), but may be equally or more likely to be retrieved on collector sites, as well as from time and experience within a field or community. Table 3.1 lists the exemplar site selected for each typology in the proposed research, and Appendix A provides visual snapshots of each website's homepage and Lexapro page.

A considerable difficulty with sampling websites on the Internet is the unknowable population of websites to sample from (Weare \& Lin, 2000). One popular 
strategy among researchers is to use search engines, such as Google and Yahoo.

Depending on the study aim, the researcher may randomly select from search returns, choose all returned sites consecutively up to a certain number, or screen sites according to certain inclusion/exclusion criteria. Search engine sampling is an arguably valid strategy because it replicates what most Internet users may retrieve and view. However, some major disadvantages to this method limited its usefulness for the present research. First, as the Internet continues to grow, the proportion of sites indexed in the major search engines decreases. It is unlikely that any combination of search engines would retrieve even half of pertinent websites. Second, as mentioned in the review of literature, search engine results for health information may be biased towards sites earning medical seals of approval for content quality (Dahlberg, 2005; Fox, 2010). Consumer-constructed health sites are typically not considered for such accolades and are, therefore, less likely to be located in the top search engine returns. Currently, major search engines like Google are specifically designing health search algorithms to return government health and medical institution websites at the top of returns, while "everything else is pushed down and out" (Fox, 2010, para. 6). Beyond open initiatives such as this, however, each search engine has unreported rules for site indexing and search retrieval, making it difficult to determine what is missing and why.

A second frequently used strategy is to base sampling on "collector sites" (Weare \& Lin, 2000). This method relies on an individual or organization with a specialized interest to collect and list links to relevant websites. The major advantage of this technique is its ability to locate obscure and special interest sites that are not retrieved by search engines, possibly for some of the reasons cited in the previous paragraph. The disadvantage of this 
technique is the existence of unknown biases resulting from non-standardized collection methods by the lists' authors.

The present study used a combination of the above strategies for selecting the 5 websites. While every attempt was made to provide clear and transparent criteria for the selection of websites, the specific selection process for each site was unique to its grouping (expert or consumer) and typology (see discussion of selection below). It is unknown whether this particular set of 5 websites would be replicated by other researchers using a similar selection method. However, with the dynamic nature and enormity of the Internet, it is unclear that any one sample would be duplicated even with a more highly consistent or systematic approach.

Selection of expert-constructed website sample.

The "professional health" type of expert-constructed site was selected based on its status as a highly-trafficked, long-established, and trusted source for the official account on illness and treatment. It was retrieved from a list of the top 20 health information websites compiled by HealthRatings.org, a joint project of Consumer Reports WebWatch and the Health Information Institute. The project ranked health information sites according to highest site traffic and then rated each of the 20 most popular sites according to 10 quality indicators. These included disclosure of ownership information, advertising and sponsorships, ease of use and accessibility, currency, privacy, coverage and content, authors and interests, references and resources, editorial policies, and health information. The site ranking highest in popularity and meeting all inclusion criteria was WebMD.com. This site scored an overall rating of "very good" and a rating of “excellent" for health information. Launched over 10 years ago, this site has a long- 
established reputation among professional health sites and has received numerous awards and recognitions from media and health fields. The site content is developed by staff writers and journalists with contributors from Medicinenet.com and reviewers from an Independent Medical Review Board. Health content covers a wide range of illnesses and conditions, treatments, strategies for healthy living, good nutrition, proper fitness, better parenting, mental health, and even pet health.

The second type of expert-constructed sites, "consumer-centric," represents a newer strand of health website that have not yet been organized or ranked in this same way. One of the first consumer-centric health site ventures was launched by an AOL cofounder in April 2007, though beta versions of the site were being tested well before the official launch ("AOL co-founder to launch revolutionhealth.com," 2007). This site, revolutionhealth.com, already has hundreds or thousands of registered users associated with any one psychotropic drug. The 2007 eHealthcare Leadership Awards named this site as "Best Overall Internet Health Site" for its excellence in providing strong health content, interactive opportunities, and good web design and navigation ("2007 web awards winners," 2008). Revolutionhealth.com exemplifies the current movement of expert-constructed health sites towards consumer-centric and Web 2.0 principles. The site boasts having " 125 online tools aimed at helping individuals take control of their wellbeing" ("About revolution health," para. 3). Health content is pulled from a network of partners, including clinics, other health news sources, and health publishers. Similar to WebMD, content on RevolutionHealth covers a wide range of illnesses, treatments, and healthy living tips and tools. Of note, both expert-constructed websites allow space for 
user-generated content, including posting of consumer ratings and reviews (see Figures A8 and A11).

\section{Selection of consumer-constructed website sample.}

Search engines, a collector site, and insight gained from field immersion were used to find consumer-constructed websites pertaining to psychotropic drugs and to select a sample that best represented a range of structures and functions. First, in February 2008 Google and Yahoo search engines were searched for one drug using the following keywords and Boolean operators: “(consumer OR patient) (discussion OR rating OR review OR support) Lexapro". This search in Google returned 130,000 hits, and in Yahoo returned 2,000,000 hits. The first 50 hits from each search engine were reviewed. Only 1 site, AskAPatient.com, returned in Google and no site returned in Yahoo met the inclusion criteria of unrestricted viewing of content and a minimum of 200 consumer contributions. AskAPatient.com was returned in Google twice, as the second and fortyfifth hit. Excluded returns from Google and Yahoo combined were: 27 links to expertconstructed health information that may have included a community forum component, 21 links to consumer-constructed websites that did not contain the minimum number of contributions for the drugs under study, 16 links to online pharmacies, 7 links to pharmaceutical company websites, 5 portal sites with advertisements, 5 government websites, 5 broken links, and 12 links to miscellaneous sites including commercial product sites, academic institutions, health insurance, and attorney solicitations.

AskAPatient.com well represents a consumer-constructed site with rigid structure and a narrowly defined function. The site has a set number of pre-defined fields for drug consumers to input comments, with the apparent function of sharing basic demographic 
information, "side effects" of medications, and a brief comment about a specific drug (see Figure A2). Other than consumer ratings and reviews, content on this site is scarce and includes only links to websites providing additional drug information and an opinion poll on a topic of the week.

The second website was selected upon reviewing a collector site called PsychCentral.com, a directory of online mental health resources created and maintained by professionals in psychology. The site has been indexing online mental health support groups for 16 years and currently receives over 650,000 visits per month. The 94 listings indexed within the site's page for "Resources" for "Medication," and 74 listings indexed within the subcategory "Support Groups" for "Mental Health" were searched. One website, CrazyMeds.us, on the medication resources page, and two websites, CrazyMeds.us and CrazyBoards.org, on the support groups for mental health page met the inclusion criteria. Both well represented the intermediate position of loose structure and fluidly defined function, though CrazyMeds.us was structured specifically around medication issues rather than general mental health issues. CrazyMeds.us was also listed as receiving a top rating on a scale from zero ("the worst") to 10 ("the best") based on 2,323 consumer votes. CrazyBoards.org received a top rating based on 416 consumer votes. CrazyMeds.us was selected for the proposed analysis because its function was more closely targeted to the purpose of this research and it appeared to receive higher site traffic based on the number of votes. Discussions on the CrazyMeds.us forum are loosely structured according to class and brand name of psychiatric drugs, with an apparent function of allowing users to discuss sometimes complicated medication issues. The creator of the website (a non-medically trained consumer) also writes a main information 
page for each drug that users are encouraged to visit prior to posting questions in the forum. Users of the CrazyMeds forum generally have a more sophisticated understanding of drug issues than might the typical consumer.

Excluded websites under the "medication resources" listings on PsychCentral.com were: 60 listings that linked to consumer reviews of specific drugs on PsychCentral.com (none of the 4 drugs under study had the minimum contributions on PsychCentral.com to be included in the analysis), 22 expert-constructed websites presenting information on specific adverse effects of drugs, 5 websites offering medication purchase assistance programs, 3 websites that did not have a minimum 200 contributions for the drugs under study, 2 forums for physician health programs, and 1 medical news site. Excluded websites under the support groups for mental health listings were: 36 websites and forums relating to general mental health issues and/or services not pertaining to the drugs under study, 20 newsgroups or mailing lists, 9 closed or private forums requiring a moderated membership, 4 websites that did not have a minimum 200 contributions for the drugs under study, 2 websites moderated by medical professionals, and 1 broken link.

The site best representing the unstructured, multi-functioning typology was selected based on the researcher's immersion in online health sites, mental health communities, and health blogs. TheIcarusProject.net is an online community of current and former psychiatric patients that stands out from other mental health communities for its relatively advanced website that includes multiple opportunities for online participation and interaction. Content on the site is largely user/community-generated, including user blogs, articles, art, as well as links to relevant news, publications, and volunteer opportunities. Pertinent to this research, the site contains a discussion forum with an 
unstructured "psychiatric medications" thread in which users can post or discuss any issue related to any psychotropic drug (see Figure A6). The function of the forum is not pre-structured, rather the users of the forum construct its function by determining what drugs and topics are discussed. This site meets all other inclusion criteria for consumerconstructed sites.

\section{Data Collection}

\section{Selected psychotropic drugs.}

Specific psychotropic drugs were used as the point of entry for all sites. If a site was not structured according to specific drugs, then text pertaining to "psychotropic drugs" served as the subject of analysis. Text regarding two psychotropic drugs from different drug classes was analyzed for each group: 1) an antidepressant, escitalopram (Lexapro), and 2) an antipsychotic, quetiapine (Seroquel), These drugs were chosen because they each appeared on the top 10 drug products of 2006 based on number of prescriptions or retail dollars. Escitalopram was approved by the FDA in 2002 and had approximately 30 million prescriptions dispensed in 2006 in the United States ("Top 10 products by U.S. dispensed prescriptions," 2007). Quetiapine was approved by the FDA in 1997 and was ranked as the ninth best-selling drug in 2006 with $\$ 3$ billion in U.S. retail sales ("Top 10 products by U.S. sales," 2007).

\section{Units of analysis.}

Existing online text pertaining to the drugs under study for each group served as the source of data. The recording unit of analysis was individual web pages within each site's root domain that contained information about the drugs under study, or "psychotropic drugs" more generally (for 1 website, see below). Each individual consumer review on 
WebMD, RevolutionHealth, and AskAPatient represented a single case for the analysis. Similarly, each individual discussion forum poster on CrazyMeds and TheIcarusProject represented a single case. For example, if AnonymousUser 35 posted a total of 10 comments about Lexapro throughout several different discussion threads on CrazyMeds, those comments were collected so that all comments by AnonymousUser 35 would represent only a single case. Expert drug information copied from expert-constructed websites also counted as one case each, creating a sample size of 4 for expert-constructed material ( 2 sites $\mathrm{x} 2$ drugs $=4$ cases $)$.

\section{Data collection procedures.}

Relevant text was copied from each website and pasted into either a Microsoft Word 2007 document or Microsoft Excel 2007 spreadsheet. All consumer reviews and discussion posts available on each website through the end of February 2009 were manually cut and pasted into the spreadsheet. In addition to consumer reviews and posts on consumer-constructed sites, the two expert-constructed sites selected for this study also allowed consumers to post individual drug reviews. Therefore, consumer reviews/posts from all 5 websites were represented in this research.

In some cases, consumers posted additional information, including their gender, age, and length of time taking the drug. When provided, these data were copied as additional columns (variables) in the spreadsheet along with the date the comment was posted and whether the consumer posted an email address or user name.

On expert-constructed sites, the relevant webpage for each drug was located using the website's internal search engine. All text pertaining to each drug, excluding advertisements and sidebar hyperlinks, was copied and pasted into a Word document. 
Consumer reviews for Lexapro and Seroquel were copied and pasted into the Excel spreadsheet.

All consumer reviews for the two drugs on AskAPatient and discussion threads on CrazyMeds were copied and pasted into the spreadsheet. TheIcarusProject discussion forum was loosely centered on "psychotropic medications," rather than containing an identifiable section specific to the drugs under study. Therefore, any discussion threads related to Lexapro or Seroquel as well as general topics involving psychotropic medications were copied and pasted into the spreadsheet. Once discussion threads from CrazyMeds and TheIcarusProject were copied, each unique poster was identified. Then, discussion posts were manually regrouped according to posters' user names so that all comments by a single user were grouped together as a single case.

To ensure proper use in the data analysis software program, all cases (consumer reviews) had to be formatted identically in the spreadsheet. To accomplish this, standardized columns were created and missing cells were filled in with "not given" for text variables and "999" for numeric variables. The variables gender, age, and length of time on the drug were transformed into categorical variables. Whether the user posted an email or user ID was a nominal (yes/no) variable. If provided, the actual email address or user ID was copied into the spreadsheet to assist with data cleaning, and was deleted prior to data analysis. Multiple comments using the same email/user ID were searched for as part of data cleaning and, when present, merged into a single comment to count as one case. Finally, the data was purged of duplicate entries.

\section{Sampling procedures.}


The data collection procedures resulted in a sampling frame of 7,418 consumer reviews/cases (see Table 3.2) and 4 expert cases. Overall, there were more consumer cases for Lexapro (62.5\%) than Seroquel (31.9\%), and more consumer cases came from expert-constructed sites (62.3\%) than consumer-constructed sites (37.7\%). Sampling from consumer cases occurred three times during this research, each by using the random numbers generator tool in Microsoft Excel. First, 85 cases from the sampling frame were randomly chosen for preliminary codebook development (see following section). Second, a coding sample of 1,080 cases was randomly chosen from the sampling frame and, finally, 216 cases from the coding sample were randomly selected for a coding agreement analysis. All 4 expert cases were coded for the analysis.

The coding sample of 1,080 consumer cases ( $14.6 \%$ of the sampling frame) was selected using a stratified simple random sample of 120 cases per drug per website (120 cases $\mathrm{x} 4$ websites x 2 drugs, plus 120 cases x 1 website). Due to the larger percentage of consumer cases found on expert-constructed sites, this sampling strategy had the effect of oversampling cases from consumer-constructed sites and under-sampling those from expert-constructed sites. This was deemed appropriate because the primary focus of this research involved a comparison between expert- and consumer-constructed websites, and an analysis of consumer reviews found on expert-constructed sites was not part of the original research proposal. Table 3.3 shows the probability of selection for any single case within each stratum.

Table 3.2 
Sampling Frame for Consumer Reviews (through February 2009)

\begin{tabular}{|c|c|c|c|c|}
\hline & Lexapro & Seroquel & $\begin{array}{c}\text { "Psychotropic } \\
\text { Drugs" }\end{array}$ & Total (\%) \\
\hline AskAPatient & 1,093 & 791 & -- & $1,884(25.4 \%)$ \\
\hline CrazyMeds & 266 & 227 & -- & $493(6.6 \%)$ \\
\hline TheIcarusProject & -- & -- & 420 & $420(5.7 \%)$ \\
\hline WebMD & 1,402 & 722 & -- & $2,124(28.6 \%)$ \\
\hline RevolutionHealth & 1,873 & 624 & -- & $2,497(33.7 \%)$ \\
\hline Total (\%) & $4,634(62.5 \%)$ & $2,364(31.9 \%)$ & $420(5.7 \%)$ & $\mathbf{7 , 4 1 8}$ \\
\hline
\end{tabular}

Table 3.3

Probability of Selection in a Stratified Simple Random Sample of 120 Cases per Drug per Website

\begin{tabular}{|c|c|c|c|}
\hline & $\begin{array}{c}\text { Lexapro } \\
(\mathrm{n} / \mathrm{N})\end{array}$ & $\begin{array}{c}\text { Seroquel } \\
(\mathrm{n} / \mathrm{N})\end{array}$ & $\begin{array}{c}\text { "Psychotropic Drugs" } \\
(\mathrm{n} / \mathrm{N})\end{array}$ \\
\hline AskAPatient & $11 \%$ & $15.2 \%$ & -- \\
\hline CrazyMeds & $45 \%$ & $52.9 \%$ & -- \\
\hline TheIcarusProject & -- & -- & $28.6 \%$ \\
\hline WebMD & $8.6 \%$ & $16.6 \%$ & -- \\
\hline RevolutionHealth & $6.4 \%$ & $19.2 \%$ & -- \\
\hline
\end{tabular}




\section{Software.}

Data coding and analysis was performed using QDA Miner version 3.2 released by Provalis Research in 2009. QDA Miner is a mixed methods data analysis software package ideal for content analysis, grounded theory, and other textual analyses. Unlike other qualitative data analysis programs, however, QDA Miner allows flexibility on the level that a case is assigned. For the present research, this meant that each consumer review could be imported from Microsoft Excel as a single case with a set of variables attached to it (i.e., age, gender, etc.). Expert cases were imported into QDA Miner from Microsoft Word. Appendix B provides 3 screenshots of coded cases in QDA Miner. Data Analysis

\section{Codebook development.}

An initial codebook was developed by coding 85 randomly selected cases from the sampling frame. This was a means to begin working with the data in preparation for the full coding sample. The end product of this phase was not a final or fixed codebook, but rather a substantial preliminary codebook that continued to evolve during subsequent stages of the coding process. In keeping with the grounded theory approach, no checklist or organizational frame was relied on to pre-structure the data. All codes were developed based on the drug effects and themes that the researcher observed in the data. However, the list of codes was eventually grouped in a logical way in a collaborative discussion between the researcher and a second coder (see subsequent section) in order to ease the coding process and facilitate a more organized analysis. Similar drug effects were grouped into a category of effects, which was determined by logic (i.e., like goes with like) as well as basic understanding of body systems (i.e., what belongs to 
musculoskeletal versus gastrointestinal systems). For example, the codes "weight gain," "weight loss," "appetite increase," and "appetite decrease" were grouped into a category called "Weight and Appetite Effects." Codes such as "constipation," "diarrhea," "nausea," and "urinary problems" were grouped into a category called "Gastrointestinal and Urinary Effects." Similarly, drug themes sharing a fundamental concept were also grouped into a category. For example, each code reflecting a strategy used to counteract medications' side effects was grouped into a category called "Dealing with Side Effects/Finding Optimum Effectiveness." The product of preliminary codebook development (i.e., the initial version of the codebook) can be found in Appendix C. The most recent version of the codebook can be found in Appendix D. Finally, more detailed descriptions of each code in the most recent codebook can be found in Appendix E.

\section{Coding agreement analysis.}

To increase reliability and credibility of findings, a coding agreement analysis was conducted for two independent coders on $20 \%$ of the coding sample. The second coder was professionally known to the lead investigator, but had not previously worked within or studied issues related to mental health or psychopharmacology. The second coder received a Masters degree in Applied Social Research in 2002 and, on this basis, was judged qualified to understand and assist in coding. This coder was first briefed on the purpose of the project and then trained in the use of the software by practice coding the 85 cases from the initial development of the codebook using the preliminary codebook developed by the lead investigator. An additional 20 cases from the sampling frame were then randomly chosen for a practice coding agreement analysis using the 
preliminary codebook. Acceptable agreement was reached (Scott's Pi $=0.77)$ on these 20 cases, and disagreements were resolved by discussion.

Once the training and practice coding for the second coder was completed, a $20 \%$ coding agreement sample (216 cases) was randomly selected from the coding sample of 1,080 cases. Inter-coder agreement was calculated in QDA Miner for each category of codes (i.e., weight gain, weight loss, appetite change were all in the category "Weight and Appetite Effects") on the level of code occurrence within a case using Scott's pi. Scott's pi is a statistical measure for inter-rater agreement of nominal level variables that takes into account chance agreements that occur from guessing (Krippendorff, 2004). It was calculated as:

\section{Q0 observad agreament - 90 axpested agramment}

1 - \% expectid agreament

While there is no firmly established rule for interpreting inter-coder agreement indices, 0.70 is often an appropriate minimum level of agreement, particularly for more conservative statistics such as Scott's pi. A Scott's pi index of at least 0.70 was prespecified in this project to indicate an acceptable level of inter-coder agreement.

However, this being a grounded theory approach to examining the data, it was expected that the codebook would continue to evolve as additional cases were reviewed. It was important that the coding agreement analysis reflected the evolving, rather than static, nature of the codebook. For this reason, the agreement coding was completed in two phases. Both coders coded the first 100 cases of the agreement sample and a Scott's Pi was calculated. The two coders together reviewed each disagreement and came to a mutual decision about its resolution. After discussion of individual coding decisions, the 
coders discussed and agreed upon revisions to the codebook (i.e., collapsing or splitting codes). Then, the same process was performed with the remaining 116 cases of the agreement sample. No changes could be made to the codebook while coding agreement was in process because both coders were required to use identical codebooks. The remaining cases in the coding sample $(\mathrm{n}=864)$ were coded by the lead investigator.

\section{Grounded theory coding.}

Coding in grounded theory refers to "naming segments of data with a label that simultaneously categorizes, summarizes, and accounts for each piece of data" (Charmaz, 2006, p. 43). Data was analyzed line-by-line for reported drug effects and themes/concepts in an iterative process of initial and focused coding. These were not distinct phases with a strict temporal sequence. The research began with initial coding but moved back-and-forth between initial and focused coding as the research progressed. Invivo codes, as opposed to researcher-derived codes, were used as frequently as possible when determining a code name. This meant that code names reflected as much as possible the language and terms of the research subjects. For example, rather than create a code called "drowsiness" or "somnolence," which reflects the standard terminology in drug literature, this study borrowed descriptive terms found in consumer comments, such as "extreme sleepiness," to serve as code names.

Initial coding stuck very close to the data and was intended to capture and condense meanings. Reported drug effects for Lexapro or Seroquel were coded only on the level of initial coding. Effects were coded literally and with as little interpretation as possible by the researcher. For example, statements such as "I was always sleepy" or "could've slept 23 hours a day" would have been coded as "excessive sleeping/tired" 
within the category "Sleep Effects." The constant comparative method of comparing data to data was used to ensure consistency in categorizing drug effects. For instance, effects such as "foggy head," "dopey" and "slowed thinking" were coded together, as were effects such as "fatigued," "tired," and "lethargic." Consistency and reliability were enhanced, then, by continually comparing new instances of an effect or variations of an effect to already coded effects.

Focused coding was intended to condense, synthesize, and explain larger segments of data using the most frequent and/or significant codes from the initial coding phase (Charmaz, 2006). Focused coding was more conceptual and served to develop the properties and dimensions of themes/concepts (Corbin \& Strauss, 2008). As part of this process, the constant comparative method was again used to compare data to data, data to codes, and codes to other codes. As additional data were coded, the description and range of each theme became more defined. Themes that began to overlap were merged together, whereas as a single theme with considerable range or variation may have been divided. For example, originally separate themes of "riding out the storm" and "waiting" were later merged into one theme because the central concept of "time" was present and overlapping in both.

Memo-writing, a conventional grounded theory technique, served as an additional window into the processes of coding. Memos are simply notes begun at the initial coding phase and intended to provide the researcher a means to track coding decisions and evolving ideas about data, codes, and concepts. They also serve as a methodological log for maintaining transparency and reflexivity in the research process (Corbin \& Strauss, 2008). In this research, memos were used at various times to 1) define and describe codes 
or themes, 2) make comparisons between data and data, data and codes, codes and codes, 3) bring raw data into the memo, 4) explain coding decisions, and 5) identify gaps in the analysis (Charmaz, 2006). For example, one gap in the analysis related to how best to describe consumers' categorization of drug effects. Memos attached to specific cases documented the tentativeness of codes such as "less severe," "severe," "best effect," or "worst effect." Other memos described the range and dimension of a theme. The code "my doctor was wrong" originally reflected consumer comments about being misinformed by their doctor, but as additional data were coded, broadened to include consumer comments about feeling uninformed after visiting their doctor, or of having a disagreement with their doctor. Memos were used to make note of these developments and refinements. All memos written during the coding process, along with the date, case number, and code are provided in Appendix F.

\section{Statistical analysis.}

Sample characteristics, reported drug effects, and themes were summarized in frequency tables. For some comparisons, such as of gender, age, and length of time on the drug across each website, empty cells prevented a statistical analysis. When cell frequencies were appropriately robust, chi-square was used in QDA Miner to test the null hypothesis that the categorical variables were independent. When possible, categories were collapsed to increase cell frequencies to 5 or higher, such as for age and length of time taking the drug. Comparisons using the chi-square test were made for: 1) gender, age, and time taken by drug, 2) commonly reported effects by website on which the comment was posted, 3) commonly reported effects by website classification as expertor consumer-constructed, 4) categories of drug effects by variables of gender, age, and 
time taken, 5) themes by website classification, 6) commonly reported effects and effect categories by time period the comment was posted, and 7) drug effect categories and themes by anonymity of the user.

For numbers 3 and 5 above, chi-square was used to test the null hypothesis that there was no association between a reported drug effect or theme and the website the effect or theme originated from. For these analyses, websites were grouped according to their status as expert-constructed (RevolutionHealth and WebMD) or consumerconstructed (AskaPatient, CrazyMeds, and IcarusProject) in order to minimize empty cells and small cell frequencies.

All significance tests were two-tailed with p-values set at $<0.05$, unless otherwise stated. In some instances and as stated in the findings, a correction was made for multiple comparisons by dividing the alpha level of 0.05 by $k$ number of comparisons.

Usability of consumer sites according to "typical" web use.

An important and practical aspect of this research related to the "usability" of consumer sites for the typical Internet user. It is difficult to duplicate or estimate a "typical" web search for any given topic because of the near infinite possible pathways to navigate the Internet. One survey reported that $41 \%$ of respondents visited 2 or 3 websites when seeking health information online, and another $20 \%$ visited 4 or 5 websites (Fox, 2006). Consumer-generated media, such as discussion forums and message boards, have become increasingly important as $34 \%$ of online health seekers reported using these resources to gather information that weighed in on their health-related decision (Elkin, 2008). It is also reasonable to assume that a "typical" online health seeker would review only a 
handful of consumers' posts on consumer review or discussion sites, rather than sifting through the full set of reviews as done in the present research.

As an attempt to preliminarily speak to the usability of consumer sites for consumers and professionals needing to make health-related decisions, the most recent 20 consumer posts from each website were extracted to examine how well they represented the full dataset of consumer reviews/posts. This analysis was limited to the 4 websites that reported drug effects: AskaPatient, CrazyMeds, RevolutionHealth, and WebMD $(n=960)$. For Crazymeds, the 20 users with the most recent posts were extracted for each drug. It was reasoned that if these 80 recent posts reported drug effects in a similar proportion to the full dataset (and depending on how the full dataset compared to expert text), then consumer reviews may be preliminarily accepted in terms of their representativeness as an additional source of information during "typical" web use. If there were significant differences in proportions of reported effects between recent posts and the full dataset, then consumers and professionals may be "harmed" by accessing incomplete information from consumer reviews during a "typical" web search. Either conclusion is preliminary as additional strategies are needed to fully understand and examine the usability issues of online consumer reviews.

\section{Preliminary data checking.}

In May 2008 as a preliminary exploration prior to data collection, nine Web 2.0 researchers, bloggers, and webmasters were contacted with inquiries as to the state-ofthe-art for identifying or preventing unauthentic accounts on sites for consumer reviews or discussion. Five responses were received. 
Computer science graduate student and developer of the Wikiscanner software, Virgil Griffith, suggested auditing Wikipedia edits of pharmaceutical companies as a way to gauge their presence in one of the most popular user-generated communities on the Internet. Wikiscanner is a free online tool used to search anonymous user edits from Wikipedia's log of IP addresses. Between February 2002 and August 2007, there were over 34.5 million anonymous edits to Wikipedia entries, making up about $21 \%$ of all Wikipedia edits during that period (Griffith, n.d.). Griffith purchased a database of over 2.6 million Internet Protocol (IP) addresses owned by organizations and found that 187,529 different organizations ( $7 \%$ of the database) contributed at least 1 anonymous Wikipedia edit. Dozens of high profile edits were immediately publicized, such as from the Federal Bureau of Investigations (FBI), Central Intelligence Agency (CIA), Walmart, Exxon, Diebold, and several others (Hafner, 2007). Also publicized and of relevance to this research topic, a person with access to the pharmaceutical company AstraZeneca's network deleted a sentence about the risk of suicidal thinking and behavior associated with the drug Seroquel (quetiapine). By 2005 Wikipedia was a top 50 website, making it likely that pharmaceutical companies may have had an interest in shaping relevant content on the site. Prior to the launch of Wikiscanner, it was also assumed that anonymous edits would remain "anonymous" in the traditional sense of "unidentified." Thus, a review of edits from pharmaceutical company networks provided a unique opportunity to gauge the activity of these organizations in popular user-generated media. As another preliminary exploration prior to data collection, Wikiscanner was used to search Astra-Zeneca, Eli Lilly, and GlaxoSmithKline for Wikipedia edits. 
Any edited entries related generally or specifically to medications or pharmaceutical companies were identified and copied into a Microsoft Word document.

Data checking of the research sample.

Consumer reviews from the present research sample were checked in an attempt to uncover possible bias that may have resulted from unauthentic consumer accounts. The first strategy addressed the hypothesis that pharmaceutical representatives may not be interested in a consumer website until it had gained some popularity among users. If a bias existed, it may appear in more recent comments. A check of the data compared early and recent comments for an imbalance of overly favorable comments in recent entries. Consumer reviews and discussion posts were divided into an early period (2002-2005) and a recent period (2006-2009) and compared across drug effect categories. The analysis of the 20 most recent posts from each website (above section) was also used to contribute to this portion of data checking.

The second strategy addressed the hypothesis that pharmaceutical representatives may prefer to contribute comments anonymously, if that option was given. A check of the data compared drug effects reported by anonymous users to user-registered/identifiable comments for an imbalance of positive reported effects in the anonymous group. CrazyMeds was excluded from this check because all users on the discussion forum had an identifiable user name. A chi-square test was used to test for an association between anonymity of the user and reported drug effects. The analysis proceeded with all data included regardless of possible bias found using these strategies. It was pre-specified that a finding of bias was to be used to add nuance to conclusions relating to the analytic 
situation - that of expertise in the age of the Internet. Such a bias was anticipated to be an important dynamic playing into those already identified in Figure 2.1.

A final check was to look for what appeared to be "scripted" comments taken from the drug manufacturer's website or downloadable medication guide. QDA Miner software was used to search the sampling frame $(7,418$ cases $)$ for 14 common phrases found on the manufacturer's drug website and medication guide for each of the two drugs being examined. The search was broadened to key words, such as "maintain* treatment," after a search of full sentences and phrases returned no results. For example, the above key words were pulled from the following sentence on Lexapro.com, "In patients who have already responded to treatment with Lexapro for their depression, maintaining treatment with Lexapro was shown to prevent the depression from returning." Retrieved entries from the 14 key words/phrases were individually scrutinized. This strategy stemmed from the comment of one consumer discussion forum owner cited previously: “They'll (pharmaceutical representatives) defend their meds if something hasn't been $100 \%$ proven, using studies that were funded by their companies or just pulling stuff out of the PI sheets. Sometimes it's as if they copied a paragraph or two from the company's website and pasted it into a post." In instances where a comment duplicated or nearly duplicated information found on the manufacturer's website or patient information sheet, the comment was excluded from analysis. Comments that appeared similar to, but did not duplicate these sources were included in the analysis. A count of the frequency of such instances was kept and used to again nuance the final conclusions. 


\section{Chapter 4: Findings}

\section{Sample Characteristics}

The coding sample consisted of 1,080 consumer reviews and discussion posts from 5 websites, and 4 expert cases from 2 websites. Most consumers on AskaPatient and WebMD reported their gender, age, and length of time on the drug (Tables 4.1, 4.3, and 4.5). The majority of consumers on the remaining 3 websites did not report gender or age, and at least half did not report length of time on the drug.

\section{Gender.}

On average and considering that $42.7 \%$ of the sample did not report gender, most consumers reported themselves as female (41.4\%). The remaining $15.9 \%$ reported themselves as male. A chi-square test of gender by drug taken (Table 4.2) showed no association between these two variables (chi-square $=1.426, \mathrm{df}=2, \mathrm{p}=0.49$ ).

Age.

Similarly, $53.5 \%$ of consumers across websites did not report their age. Approximately $33 \%$ of consumers reported an age between 25 and 54 years old, with a slight majority (13.4\%) of those consumers reporting to be in the younger 25-34 bracket. Another $8.5 \%$ of consumers reported an age less than 24 years, and just less than $5 \%$ reported an age over 55 years. A chi-square test of age by drug taken (Table 4.4) did show an association between the two variables (chi-square $=14.355, \mathrm{df}=6, \mathrm{p}=0.026$ ). Consumers in the younger age groups of 7-18 and 25-34 reported slightly higher use of Seroquel, whereas a higher percentage of consumers aged 19-24 and 35-44 were taking Lexapro. 


\section{Length of time on drug.}

Approximately $35 \%$ of the sample did not report the length of time taking

Lexapro or Seroquel, but 37\% reported taking the drug for less than 6 months (Table 4.5). Another $18 \%$ reported taking the drug between 1 and 5 years, and less than $2.5 \%$ reported taking it for more than 5 years. A chi-square test (Table 4.6) of this variable by drug taken did show an association (chi-square $=20.423, \mathrm{df}=6, \mathrm{p}=0.002$ ). Consumers taking the drug for less than 6 months were more often using Lexapro, while consumers taking the drug for 2 years or more were more often using Seroquel.

\section{Year posted.}

Consumer reviews were posted as early as 2002 on RevolutionHealth, 2003 on AskaPatient, CrazyMeds, and IcarusProject, and 2007 on WebMD (Table 4.7). More than $68 \%$ of consumer reviews were posted from 2006-2008. Approximately $24 \%$ of reviews were posted in or before 2005, and nearly two-thirds of those came from RevolutionHealth. Table 4.8 shows there were also more reviews for Lexapro than Seroquel in 2002-2003 (Lexapro received FDA approval in 2002), and more reviews for Seroquel in 2004-2005 (Seroquel received FDA approval for a second indication in 2004).

\section{User ID/email.}

More than half (55.7\%) of consumers did provide a user ID or email address with their review or post (Table 4.9), however, this number reflected the fact that all users on CrazyMeds and IcarusProject have a unique user ID for posting and for receiving private messages from other users. Table 4.10 shows a slightly higher proportion of consumers using Seroquel provided a user ID or email address. 
Table 4.1

Number of Consumers Reporting Gender across All Websites

\begin{tabular}{|r|c|c|c|c|c|c|}
\hline & $\begin{array}{c}\text { Askapatient } \\
(\mathrm{N}=240)\end{array}$ & $\begin{array}{c}\text { CrazyMeds } \\
(\mathrm{N}=240)\end{array}$ & $\begin{array}{c}\text { IcarusProject } \\
(\mathrm{N}=120)\end{array}$ & $\begin{array}{c}\text { RevHealth } \\
(\mathrm{N}=240)\end{array}$ & $\begin{array}{c}\text { WebMD } \\
(\mathrm{N}=240)\end{array}$ & $\begin{array}{c}\text { Total } \\
\mathbf{( \% )}\end{array}$ \\
\hline Female & 158 & 64 & 25 & 22 & 179 & $\begin{array}{c}448 \\
(41.4 \%)\end{array}$ \\
\hline Male & 81 & 27 & 4 & 10 & 50 & $\begin{array}{c}172 \\
(15.9 \%)\end{array}$ \\
\hline $\begin{array}{r}\text { Niven } \\
\text { Nivet }\end{array}$ & $1(0.4 \%)$ & $\begin{array}{c}149 \\
(62.1 \%)\end{array}$ & $91(75.8 \%)$ & $\begin{array}{c}208 \\
(86.7 \%)\end{array}$ & $\begin{array}{c}11 \\
(4.6 \%)\end{array}$ & $\begin{array}{c}462 \\
(42.7 \%)\end{array}$ \\
\hline
\end{tabular}

Table 4.2

Number of Consumers Reporting Gender According to Drug Taken ${ }^{a}$

\begin{tabular}{|r|c|c|}
\hline & $\begin{array}{c}\text { Lexapro (\%) } \\
(\mathrm{N}=480)\end{array}$ & $\begin{array}{c}\text { Seroquel (\%) } \\
(\mathrm{N}=480)\end{array}$ \\
\hline Female & $216(45.0 \%)$ & $207(43.1 \%)$ \\
\hline Male & $77(16.0 \%)$ & $91(19.0 \%)$ \\
\hline Not Given & $187(39.0 \%)$ & $182(37.9 \%)$ \\
\hline
\end{tabular}

${ }^{\mathrm{a}}$ Chi-square $=1.426, \mathrm{df}=2, \mathrm{p}=0.49$ 
Table 4.3

Number of Consumers Reporting Age across All Websites

\begin{tabular}{|c|c|c|c|c|c|c|}
\hline Age & $\begin{array}{l}\text { Askapatient } \\
\qquad(\mathrm{N}=240)\end{array}$ & $\begin{array}{c}\text { CrazyMeds } \\
(\mathrm{N}=240)\end{array}$ & $\begin{array}{l}\text { IcarusProject } \\
\qquad(\mathrm{N}=120)\end{array}$ & $\begin{array}{l}\text { RevHealth } \\
(\mathrm{N}=240)\end{array}$ & $\begin{array}{l}\text { WebMD } \\
(\mathrm{N}=240)\end{array}$ & Total (\%) \\
\hline $7-12$ & 2 & 1 & & 1 & 1 & $5(0.5 \%)$ \\
\hline $13-18$ & 13 & 2 & 1 & 1 & 4 & $21(1.9 \%)$ \\
\hline $19-24$ & 41 & 1 & 2 & & 22 & $66(6.1 \%)$ \\
\hline $25-34$ & 75 & 2 & 5 & 3 & 60 & $\begin{array}{c}145 \\
(13.4 \%)\end{array}$ \\
\hline $35-44$ & 52 & 6 & & 2 & 50 & $\begin{array}{c}110 \\
(10.2 \%)\end{array}$ \\
\hline $45-54$ & 41 & 4 & 2 & 4 & 53 & $\begin{array}{c}104 \\
(9.6 \%)\end{array}$ \\
\hline $55-64$ & 11 & 1 & & 1 & 32 & $45(4.2 \%)$ \\
\hline $65-74$ & 1 & 1 & & & 3 & $5(0.5 \%)$ \\
\hline $75+$ & 1 & & & & 1 & $2(0.2 \%)$ \\
\hline $\begin{array}{l}\text { not } \\
\text { given }\end{array}$ & $3(1.3 \%)$ & $\begin{array}{c}224 \\
(92.6 \%)\end{array}$ & $110(91.7 \%)$ & $228(95 \%)$ & $\begin{array}{c}14 \\
(5.8 \%)\end{array}$ & $\begin{array}{c}579 \\
(53.5 \%)\end{array}$ \\
\hline
\end{tabular}


Table 4.4

Number of Consumers Reporting Age According to Drug Taken ${ }^{a}$

\begin{tabular}{|r|c|c|}
\hline \multicolumn{2}{|r|}{ Age } & $\begin{array}{c}\text { Lexapro (\%) } \\
(\mathrm{N}=480)\end{array}$ \\
\hline $7-18^{\mathrm{b}}$ & $7(1.5 \%)$ & $18(3.8 \%)$ \\
\hline $19-24$ & $38(7.9 \%)$ & $26(5.4 \%)$ \\
\hline $25-34$ & $64(13.3 \%)$ & $76(15.8 \%)$ \\
\hline $35-44$ & $67(14.0 \%)$ & $43(9.0 \%)$ \\
\hline $45-54$ & $46(10.0 \%)$ & $56(11.7 \%)$ \\
\hline $55+{ }^{\mathrm{b}}$ & $26(4.4 \%)$ & $26(5.0 \%)$ \\
\hline not given & $232(48.3 \%)$ & $235(49.0 \%)$ \\
\hline
\end{tabular}

${ }^{\mathrm{a}}$ Chi-square $=14.355, \mathrm{df}=6, \mathrm{p}=0.026$

${ }^{\mathrm{b}}$ Categories were collapsed for this analysis. 
Table 4.5

Number of Consumers Reporting Length of Time on the Drug across All Websites

\begin{tabular}{|c|c|c|c|c|c|c|}
\hline $\begin{array}{l}\text { Time } \\
\text { Taken }\end{array}$ & $\begin{array}{l}\text { Askapatient } \\
\qquad(\mathrm{N}=240)\end{array}$ & $\begin{array}{l}\text { CrazyMeds } \\
\qquad(\mathrm{N}=240)\end{array}$ & $\begin{array}{l}\text { IcarusProject } \\
\qquad(\mathrm{N}=120)\end{array}$ & $\begin{array}{l}\text { RevHealth } \\
(\mathrm{N}=240)\end{array}$ & $\begin{array}{l}\text { WebMD } \\
(\mathrm{N}=240)\end{array}$ & $\begin{array}{c}\text { Total } \\
(\%)\end{array}$ \\
\hline$<1 \mathrm{mth}$ & 67 & 23 & 3 & 43 & 51 & $\begin{array}{c}187 \\
(17.3 \%)\end{array}$ \\
\hline $\begin{array}{r}1-6 \\
\text { mths }\end{array}$ & 72 & 38 & 2 & 42 & 59 & $\begin{array}{c}213 \\
(19.7 \%)\end{array}$ \\
\hline $\begin{array}{r}6 \mathrm{mths}- \\
1 \mathrm{yr}\end{array}$ & 29 & 16 & 1 & 11 & 28 & $\begin{array}{c}85 \\
(7.9 \%)\end{array}$ \\
\hline $1-2$ yrs & 29 & 21 & & 6 & 27 & $\begin{array}{c}83 \\
(7.7 \%)\end{array}$ \\
\hline $2-5 \mathrm{yrs}$ & 34 & 20 & 2 & 8 & 46 & $\begin{array}{c}110 \\
(10.2 \%)\end{array}$ \\
\hline $5-10$ yrs & 7 & 4 & & 2 & 11 & $\begin{array}{c}24 \\
(2.2 \%)\end{array}$ \\
\hline $10 \mathrm{yrs}^{+}$ & 1 & & & & 1 & $2(0.2 \%)$ \\
\hline $\begin{array}{r}\text { not } \\
\text { given }\end{array}$ & $\begin{array}{c}1 \\
(0.4 \%)\end{array}$ & $\begin{array}{c}120 \\
(49.6 \%)\end{array}$ & $\begin{array}{c}112 \\
(93.3 \%)\end{array}$ & $\begin{array}{c}128 \\
(53.3 \%)\end{array}$ & $\begin{array}{c}17 \\
(7.1 \%)\end{array}$ & $\begin{array}{c}378 \\
(34.9 \%)\end{array}$ \\
\hline
\end{tabular}


Table 4.6

Number of Consumers Reporting Length of Time on the Drug according to Drug Taken ${ }^{a}$

\begin{tabular}{|r|c|c|}
\hline \multicolumn{1}{|l|}{ Time Taken } & $\begin{array}{c}\text { Lexapro }(\%) \\
(\mathrm{N}=480)\end{array}$ & $\begin{array}{c}\text { Seroquel }(\%) \\
(\mathrm{N}=480)\end{array}$ \\
\hline$<1 \mathrm{mth}$ & $106(22.1 \%)$ & $78(16.2 \%)$ \\
\hline $1-6 \mathrm{mths}$ & $122(25.4 \%)$ & $42(8.6 \%)$ \\
\hline $6 \mathrm{mths}-1 \mathrm{yr}$ & $42(8.6 \%)$ & $40(8.3 \%)$ \\
\hline $1-2 \mathrm{yrs}$ & $43(9.0 \%)$ & $62(12.9 \%)$ \\
\hline $2-5 \mathrm{yrs}$ & $46(9.6 \%)$ & $17(3.5 \%)$ \\
\hline $5 \mathrm{yrs}+{ }^{\mathrm{b}}$ & $9(1.9 \%)$ & $152(31.7 \%)$ \\
\hline not given & $112(23.3 \%)$ & \\
\hline
\end{tabular}

${ }^{\mathrm{a}}$ Chi-square $=20.423, \mathrm{df}=6, \mathrm{p}=0.002$

${ }^{\mathrm{b}}$ The categories 5-10yrs and 10yrs + were collapsed for this analysis. 
Table 4.7

Number of Consumer Posts According to Year Posted across all Websites

\begin{tabular}{|c|c|c|c|c|c|c|}
\hline $\begin{array}{r}\text { Year } \\
\text { Posted }\end{array}$ & $\begin{array}{l}\text { Askapatient } \\
(\mathrm{N}=240)\end{array}$ & $\begin{array}{l}\text { CrazyMeds } \\
\qquad(\mathrm{N}=240)\end{array}$ & $\begin{array}{l}\text { IcarusProject } \\
\qquad(\mathrm{N}=120)\end{array}$ & $\begin{array}{l}\text { RevHealth } \\
(\mathrm{N}=240)\end{array}$ & $\begin{array}{l}\text { WebMD } \\
(\mathrm{N}=240)\end{array}$ & $\begin{array}{c}\text { Total } \\
(\%)\end{array}$ \\
\hline 2002 & & & & 19 & & $19(1.8 \%)$ \\
\hline 2003 & 2 & 1 & 7 & 36 & & $46(4.3 \%)$ \\
\hline 2004 & 15 & 1 & 23 & 48 & & $87(8.0 \%)$ \\
\hline 2005 & 26 & & 23 & 57 & & $\begin{array}{c}106 \\
(9.8 \%)\end{array}$ \\
\hline 2006 & 47 & & 26 & 68 & & $\begin{array}{c}141 \\
(13.0 \%)\end{array}$ \\
\hline 2007 & 81 & 88 & 26 & 6 & 52 & $\begin{array}{c}253 \\
(23.4 \%)\end{array}$ \\
\hline 2008 & 52 & 127 & 15 & 3 & 148 & $\begin{array}{c}347 \\
(32.1 \%)\end{array}$ \\
\hline $2009^{\mathrm{a}}$ & 17 & 25 & & 3 & 40 & $85(7.9 \%)$ \\
\hline
\end{tabular}

${ }^{\mathrm{a}}$ Data in this row represents January and February 2009. 
Table 4.8

Number of Consumer Posts for Each Drug According to Year Posted

\begin{tabular}{|r|c|c|}
\hline Year Posted & $\begin{array}{c}\text { Lexapro (\%) } \\
(\mathrm{N}=480)\end{array}$ & $\begin{array}{c}\text { Seroquel (\%) } \\
(\mathrm{N}=480)\end{array}$ \\
\hline 2002 & $17(3.5 \%)$ & $2(0.4 \%)$ \\
\hline 2003 & $25(5.2 \%)$ & $13(2.7 \%)$ \\
\hline 2004 & $22(4.6 \%)$ & $41(8.5 \%)$ \\
\hline 2005 & $31(6.5 \%)$ & $52(10.8 \%)$ \\
\hline 2006 & $57(11.9 \%)$ & $58(12.1 \%)$ \\
\hline 2007 & $117(24.4 \%)$ & $110(22.9 \%)$ \\
\hline 2008 & $163(34.0 \%)$ & $167(34.8 \%)$ \\
\hline $2009^{a}$ & $48(10.0 \%)$ & $37(7.7 \%)$ \\
\hline
\end{tabular}

${ }^{\mathrm{a}}$ Data in this row represents January and February 2009.

Table 4.9

Number of Consumers Who Provided a User ID or Email Address across All Websites

\begin{tabular}{|c|c|c|c|c|c|c|}
\hline & $\begin{array}{l}\text { Askapatient } \\
(\mathrm{N}=240)\end{array}$ & $\begin{array}{c}\text { CrazyMeds } \\
(\mathrm{N}=240)\end{array}$ & $\begin{array}{l}\text { IcarusProject } \\
\qquad(\mathrm{N}=120)\end{array}$ & $\begin{array}{c}\text { RevHealth } \\
(\mathrm{N}=240)\end{array}$ & $\begin{array}{l}\text { WebMD } \\
(\mathrm{N}=240)\end{array}$ & $\begin{array}{c}\text { Total } \\
(\%)\end{array}$ \\
\hline $\begin{array}{r}\text { (YES) } \\
\text { ID/email } \\
\text { was given }\end{array}$ & 60 & 240 & 120 & 33 & 150 & $\begin{array}{c}603 \\
(55.7 \%)\end{array}$ \\
\hline $\begin{array}{l}\text { (No) ID/ } \\
\text { email was } \\
\text { not given }\end{array}$ & 180 & & & 207 & 90 & $\begin{array}{c}477 \\
(44.1 \%)\end{array}$ \\
\hline
\end{tabular}


Table 4.10

Number of Consumers Who Provided a User ID or Email Address According to Drug Taken

\begin{tabular}{|r|c|c|}
\hline & $\begin{array}{c}\text { Lexapro (\%) } \\
(\mathrm{N}=480)\end{array}$ & $\begin{array}{c}\text { Seroquel (\%) } \\
(\mathrm{N}=480)\end{array}$ \\
\hline (YES) ID/email was given & $226(47.1 \%)$ & $257(53.5 \%)$ \\
\hline (No) ID/email was not given & $254(52.9 \%)$ & $223(46.5 \%)$ \\
\hline
\end{tabular}

Inter-Coder Agreement Analysis

Two coders coded a random sample of 216 cases in two phases $(n=100$ in phase 1 , $\mathrm{n}=116$ in phase 2 ). Table 4.11 shows the results of the inter-coder agreement analysis. Acceptable overall agreement was found in both phases (Scott's $\mathrm{Pi}=0.756$ in phase 1 , Scott's $\mathrm{Pi}=0.751$ in phase 2 ). In general, agreement was higher for drug effects than for themes and concepts. After each phase of agreement analysis, the two coders discussed and resolved disagreements (see Appendix G). Many of the coding disagreements were traced to a lack of clarity or understanding by the second coder. For example, upon reviewing the codes of both coders, it became clear that the second coder was unsure when to appropriately apply codes within the category "Evidence of Causality" because of a misunderstanding about the purpose of those codes. The second coder also had difficulty applying codes for "Discontinuing/Missed Doses" due to a lack of knowledge of what "discontinuing" meant. In other cases, such as for "Other Drug Issues" and "Support and Advice," the second coder applied these codes more liberally than the principal investigator, despite the provided descriptions of the concepts each of these 
codes encompassed. Finally on a few occasions, such as for "Other Effects" of the drugs, and as part of the process of grounded theory coding, the principal investigator began applying codes to text segments that broadened the scope of the code. For example, the code "no/limited side effects" which falls under the "Other Effects" category, was initially defined to only include comments that clearly stated there were no side effects of the drug. During coding, however, the principal investigator observed many comments that seemed to define drug effects according to the absence (rather than the presence) of a specific effect (i.e., "I've experienced no weight gain on this drug"). These comments were coded by the principal investigator as "no/limited side effects," and often left uncoded by the second coder.

The remaining coding and analysis proceeded as planned because the overall intercoder agreement statistic was above the minimally acceptable level of 0.70 , and because most coding disagreements were related to 1) a lack of clarity or understanding of terminology and/or code definitions by the second coder, and 2) the evolving nature of the grounded theory coding method in which codes continued to expand and collapse throughout the process. The coders agreed that there did not appear to be unfounded leaps from data to codes. Revisions to the codebook to clarify codes and ease the coding process were made after each phase of the agreement analysis based upon the two coders' discussions. 
Table 4.11

Inter-coder Agreement Analysis

\begin{tabular}{|c|c|c|}
\hline & $\begin{array}{c}\text { Scott's Pi, part } \\
1 \\
(\mathrm{~N}=100)\end{array}$ & $\begin{array}{c}\text { Scott's Pi, part } \\
2 \\
(\mathrm{~N}=116)\end{array}$ \\
\hline \multicolumn{3}{|l|}{ Drug Effects } \\
\hline Appetite and Weight Effects & 1 & 0.82 \\
\hline Gatrointestinal and Urinary Effects & 0.851 & 1 \\
\hline Head/Face Effects & 0.889 & 0.683 \\
\hline Lab Tests and Chronic Conditions & 1 & 0.884 \\
\hline Mental or Mood Effects & 0.889 & 0.809 \\
\hline Musculoskeletal and Neurological Effects & 0.826 & 0.811 \\
\hline Nose/Throat/Chest Effects & 0.591 & 0.81 \\
\hline Other Effects & 0.632 & 0.361 \\
\hline Sexual Effects & 0.852 & 1 \\
\hline Skin Effects & 0.949 & 0.653 \\
\hline Sleep Effects & 0.928 & 0.913 \\
\hline \multicolumn{3}{|l|}{ Themes/Concepts } \\
\hline Assessing the Overall Experience & 0.476 & 0.628 \\
\hline Citations and Links & 1 & 0.633 \\
\hline Classification of Effects & 0.202 & 0.644 \\
\hline Contraindications & -0.005 & 0.791 \\
\hline $\begin{array}{l}\text { Dealing with Side Effects/Finding Optimum } \\
\text { Effectiveness }\end{array}$ & 0.668 & 0.723 \\
\hline
\end{tabular}




\begin{tabular}{|l|c|c|}
\hline & $\begin{array}{c}\text { Scott's Pi, part } \\
1 \\
(\mathrm{~N}=100)\end{array}$ & $\begin{array}{c}\text { Scott's Pi, part } \\
2 \\
(\mathrm{~N}=116)\end{array}$ \\
\hline Discontinuing/Missed Doses & 1 & 0.598 \\
\hline Evidence of Causality & 0.333 & 0.504 \\
\hline Finances / The System & 0.918 & 0.948 \\
\hline Friends and Family & 0.556 & 0.646 \\
\hline My Diagnosis/Reason for Use & 0.74 & 0.69 \\
\hline My Doctor & 0.645 & 0.741 \\
\hline Other Drug Issues & 0.904 & 0.563 \\
\hline Other Drugs and Drug Combinations & 0.485 & 0.822 \\
\hline Pharmaceutical Companies/FDA & 0.398 & 0.597 \\
\hline Support and Advice & 0.936 & 0.56 \\
\hline Theories of Drug Action & 0.668 & 0.741 \\
\hline TOTAL & $\mathbf{0 . 7 5 6}$ & $\mathbf{0 . 7 5 1}$ \\
\hline
\end{tabular}

\section{Drug Effects According to Consumers}

Overview of drug effect categories.

The most recent codebook in Appendix D shows 11 categories of drug effects. Effects were grouped broadly in order to facilitate robust analyses. The categories that resulted were not based on a standardized medical dictionary, and the meaning of the specific groupings should not be exaggerated in the final conclusions. In most cases where categories were used for analysis, individual effects were further examined to determine where differences lay. 
Appetite and weight effects were grouped in their own category, paralleling what might be called metabolic effects in a standardized dictionary. These effects appeared frequently enough in the data to warrant their own category, rather than be grouped with, for example, gastrointestinal effects. Similarly, sexual effects, which could have alternatively been grouped under genito-urinary effects, and sleep effects recurred frequently enough to merit autonomous categories. At the same time, there were few instances of other genito-urinary effects (i.e., involving urination and menstruation), so these were combined with gastrointestinal effects (i.e., nausea/vomiting, constipation, etc.) to create a single category called gastrointestinal and urinary effects.

Head/face effects involved physical effects of those body parts, such as headache and jaw clenching. Codes under nose/throat/chest effects and skin effects were similarly grouped. Again, these were grouped broadly, rather than broken down into smaller categories of respiratory effects, sensory effects, and so on. Mental or mood effects included all non-physical, psychological and behavioral drug effects.

Musculoskeletal and neurological effects were grouped into 1 category because of slight overlap in these effects, such as for tingling sensations and numbness which could be reasonably classified under either body system. Also, there were too few effects within either category alone to stand on its own.

The category "lab tests and chronic conditions," referred to effects that show up only on laboratory tests, such as high blood sugar or cholesterol, and that may cause chronic disease conditions, such as diabetes. These were grouped together, rather than distributed across various body systems, because previous research in this area found that consumers 
online less frequently mentioned these types of effects specifically as compared to information provided in expert sources.

Finally, "other effects" included codes that were infrequently mentioned and could not be otherwise classified.

\section{Lexapro.}

Table 4.12 shows the percentage of consumers mentioning an effect grouped by the category of effects. For example, the category "sexual effects" included codes for lost sex drive, trouble with orgasm, and other sexual effects.. "Mental or mood effects" was the most commonly reported category of effects with almost $62 \%$ of consumers mentioning them.

Table 4.13 lists all effects of Lexapro mentioned by at least $3 \%$ of consumers. The top 4 most mentioned effects were related to symptoms and sleep. Approximately $30 \%$ of consumers reported improvement in symptoms, such as anxiety, depression, or mania, as an effect of Lexapro. Another $15.8 \%$ reported new or worsening symptoms as an effect of Lexapro, including new or worsened panic attacks, depression, or mania. Sleep changes ranging from excessive sleepiness to insomnia were mentioned by about $24 \%$ and $13 \%$ of consumers, respectively. All other effects mentioned could be considered "side effects."

Figure 4.1 and Table 4.14 show the 9 most reported effects for Lexapro across 4 websites, measured by code occurrence in a case (posts from IcarusProject were not included in analyses of drug effects). Figure 4.1 visually displays the lack of consistency of consumer reporting across these websites. With p-values set at 0.001 for multiple comparisons, statistically significant chi-square coefficients were reached for half of these effects, indicating that there was an association between symptom improvement, 
extreme sleepiness, nausea/vomiting, and lost sex drive, and the website the post originated. Compared to the other 3 websites, WebMD showed a relatively low number of consumers mentioning what are usually considered "side effects" of the drug, including sleep changes, new or worsened symptoms, brain fog/zombie, weight gain, and nausea/vomiting. Across all 9 top reported effects, consumers on AskaPatient reported these effects in high numbers, whereas consumers on CrazyMeds reported a comparatively low number of drug effects. RevolutionHealth had the highest percentage of consumers mentioning symptom improvement compared to the other 3 websites, and the lowest percentage of consumers mentioning weight gain. For the remaining listed effects, consumers on RevolutionHealth provided varied reports.

In Table 4.15, Lexapro effects mentioned by at least $3 \%$ of consumers are compared across consumer-constructed websites (AskaPatient and CrazyMeds) and expertconstructed websites (RevolutionHealth and WebMD). Only 1 of the 28 effects listed reached statistically significant p-values at the 0.001 level (dreams vivid/nightmares), indicating that for a majority of effects there was no association between the effect and the classification of the website as consumer- or expert-constructed. More consumers posting on expert-constructed websites reported Lexapro improved their symptoms ( $\mathrm{p}=.037)$, while more consumers on consumer-constructed websites reported the drug worsened or induced new symptoms $(\mathrm{p}=.034)$. All effects that would be popularly labeled as side effects or adverse effects were each mentioned more by consumers on consumerconstructed rather than expert-constructed websites, with the exception of loss of appetite. 
Table 4.12

Top 5 Effect Categories Mentioned by Consumers for Lexapro

\begin{tabular}{|r|c|}
\hline \multicolumn{1}{|c|}{ Category of effects } & \% of consumers mentioning the effect \\
\hline Mental or mood effects & $61.7 \%$ \\
\hline Sleep effects & $36.0 \%$ \\
\hline Appetite and weight effects & $22.5 \%$ \\
\hline Sexual effects & $20.2 \%$ \\
\hline
\end{tabular}

Table 4.13

Effects of Lexapro Mentioned by at Least 3\% of Consumers

\begin{tabular}{|l|c|}
\hline \multicolumn{1}{|c|}{ Drug effect } & \% of consumers mentioning the effect \\
& $(\mathrm{N}=480)$ \\
\hline Symptoms reduced / improved & $30.4 \%$ \\
\hline Extreme sleepiness / tired & $23.8 \%$ \\
\hline Symptoms new / worsened & $15.8 \%$ \\
\hline Trouble sleeping / insomnia & $13.3 \%$ \\
\hline Emotionally stable / calm / "normal" & $13.1 \%$ \\
\hline Weight gain & $13.1 \%$ \\
\hline Nausea / sick stomach / vomiting & $12.1 \%$ \\
\hline $\begin{array}{l}\text { Numb / detached / lack of energy or } \\
\text { interest }\end{array}$ & $11.3 \%$ \\
\hline Brain fog / zombie & $10.8 \%$ \\
\hline Lost sex drive/low libido & $10.6 \%$ \\
\hline
\end{tabular}




\begin{tabular}{|l|c|}
\hline \multicolumn{1}{|c|}{ Drug effect } & $\begin{array}{c}\text { \% of consumers mentioning the effect } \\
(\mathrm{N}=480)\end{array}$ \\
\hline Trouble achieving orgasm & $8.5 \%$ \\
\hline Headache increased & $7.1 \%$ \\
\hline Jaw clenching / grinding & $6.9 \%$ \\
\hline Energy increased / euphoria / mania & $6.9 \%$ \\
\hline "Sweating like a pig" & $6.5 \%$ \\
\hline Dreams vivid / nightmares & $6.3 \%$ \\
\hline Abnormal movements & $5.6 \%$ \\
\hline Agitated / restless & $5.4 \%$ \\
\hline Loss of appetite & $4.8 \%$ \\
\hline Weight loss & $4.8 \%$ \\
\hline Other misc effects & $4.6 \%$ \\
\hline Dizzy / lightheaded / faint & $4.6 \%$ \\
\hline Diarrhea / gas & $4.6 \%$ \\
\hline Dry mouth / sore throat & $4.4 \%$ \\
\hline Increase appetite / cravings & $4.2 \%$ \\
\hline $\begin{array}{l}\text { Ability to do things in absence of } \\
\text { symptoms }\end{array}$ & $3.1 \%$ \\
\hline Yawning & \\
\hline Other sexual effects & \\
\hline
\end{tabular}


Figure 4.1 Most Popularly Mentioned Lexapro Effects by Consumers across Websites

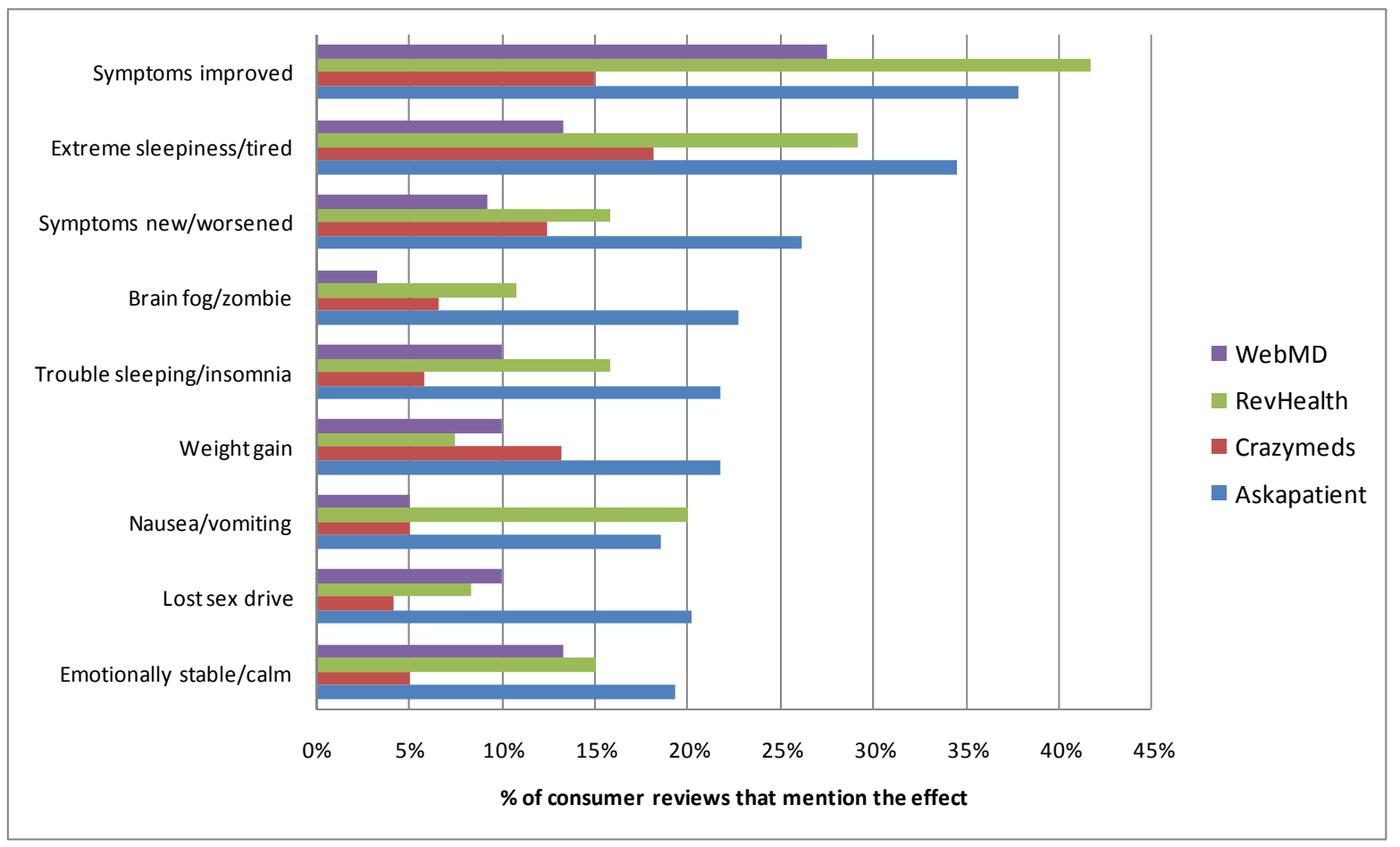


Table 4.14

Number of Consumers on each Website Mentioning the Most Popularly Reported Effects of Lexapro $^{a}$

\begin{tabular}{|c|c|c|c|c|c|c|}
\hline & $\begin{array}{c}\text { AskaPatient } \\
\mathrm{N}=120\end{array}$ & $\begin{array}{c}\text { CrazyMeds } \\
\qquad \mathrm{N}=120\end{array}$ & $\begin{array}{l}\text { RevHealth } \\
\qquad \mathrm{N}=120\end{array}$ & $\begin{array}{l}\text { WebMD } \\
\mathrm{N}=120\end{array}$ & $\begin{array}{l}\text { Chi- } \\
\text { square }\end{array}$ & $\begin{array}{c}\mathrm{p}- \\
\text { value }\end{array}$ \\
\hline $\begin{array}{l}\text { Symptoms } \\
\text { improved }\end{array}$ & 45 & 18 & 50 & 33 & 24.543 & 0.000 \\
\hline $\begin{array}{l}\text { Extreme } \\
\text { sleepiness/tired }\end{array}$ & 41 & 22 & 35 & 16 & 18.735 & 0.000 \\
\hline $\begin{array}{l}\text { Symptoms new/ } \\
\text { worsened }\end{array}$ & 31 & 15 & 19 & 11 & 14.396 & 0.002 \\
\hline Brain fog/zombie & 27 & 8 & 13 & 4 & -- & -- \\
\hline $\begin{array}{l}\text { Trouble sleeping } \\
\text { /insomnia }\end{array}$ & 26 & 7 & 19 & 12 & 15.236 & 0.002 \\
\hline Weight gain & 26 & 16 & 9 & 12 & 12.301 & 0.006 \\
\hline $\begin{array}{l}\text { Nausea/sick } \\
\text { stomach/ vomiting }\end{array}$ & 22 & 6 & 24 & 6 & 23.123 & 0.000 \\
\hline $\begin{array}{l}\text { Lost sex } \\
\text { drive/libido }\end{array}$ & 24 & 5 & 10 & 12 & 17.497 & 0.001 \\
\hline $\begin{array}{l}\text { Emotionally } \\
\text { stable/calm/ } \\
\text { "normal" }\end{array}$ & 23 & 6 & 18 & 16 & 11.467 & 0.009 \\
\hline
\end{tabular}

${ }^{\mathrm{a}} \mathrm{p}$-value set at 0.001 for $\mathrm{k}=35$ comparisons in Tables 4.14 and 4.15 
Table 4.15

Lexapro Effects Mentioned by $>3 \%$ of Consumers according to Website Classification ${ }^{a}$

\begin{tabular}{|c|c|c|c|c|}
\hline Drug effect & $\begin{array}{l}\text { Consumer- } \\
\text { constructed } \\
(\mathrm{N}=220)\end{array}$ & $\begin{array}{c}\text { Expert- } \\
\text { constructed } \\
(\mathrm{N}=220)\end{array}$ & Chi-square & p-value \\
\hline $\begin{array}{l}\text { Symptoms reduced / } \\
\text { improved }\end{array}$ & 62 & 83 & 4.358 & 0.037 \\
\hline $\begin{array}{l}\text { Extreme sleepiness / } \\
\text { tired }\end{array}$ & 62 & 51 & 1.400 & 0.237 \\
\hline $\begin{array}{l}\text { Symptoms new / } \\
\text { worsened }\end{array}$ & 47 & 30 & 4.470 & 0.034 \\
\hline $\begin{array}{l}\text { Trouble sleeping / } \\
\text { insomnia }\end{array}$ & 32 & 31 & 0.018 & 0.892 \\
\hline $\begin{array}{l}\text { Emotionally stable / } \\
\text { calm / "normal" }\end{array}$ & 29 & 34 & 0.457 & 0.499 \\
\hline Weight gain & 41 & 21 & 7.409 & 0.006 \\
\hline $\begin{array}{l}\text { Nausea / sick } \\
\text { stomach / vomiting }\end{array}$ & 27 & 30 & 0.179 & 0.672 \\
\hline $\begin{array}{l}\text { Numb / detached / } \\
\text { lack of energy or } \\
\text { interest }\end{array}$ & 31 & 22 & 1.718 & 0.19 \\
\hline Brain fog / zombie & 34 & 17 & 6.340 & 0.012 \\
\hline $\begin{array}{l}\text { Lost sex drive/low } \\
\text { libido }\end{array}$ & 28 & 22 & 0.804 & 0.37 \\
\hline $\begin{array}{l}\text { Trouble achieving } \\
\text { orgasm }\end{array}$ & 24 & 16 & 1.745 & 0.186 \\
\hline Headache increased & 20 & 13 & 1.594 & 0.207 \\
\hline $\begin{array}{l}\text { Jaw clenching / } \\
\text { grinding }\end{array}$ & 23 & 9 & 6.653 & 0.01 \\
\hline
\end{tabular}




\begin{tabular}{|c|c|c|c|c|}
\hline Drug effect & $\begin{array}{c}\text { Consumer- } \\
\text { constructed } \\
(\mathrm{N}=220)\end{array}$ & $\begin{array}{c}\text { Expert- } \\
\text { constructed } \\
(\mathrm{N}=220)\end{array}$ & Chi-square & p-value \\
\hline $\begin{array}{l}\text { Energy increased / } \\
\text { euphoria / mania }\end{array}$ & 17 & 15 & 0.134 & 0.714 \\
\hline "Sweating like a pig" & 19 & 11 & 2.276 & 0.131 \\
\hline $\begin{array}{l}\text { Dreams vivid / } \\
\text { nightmares }\end{array}$ & 25 & 5 & 14.222 & 0.000 \\
\hline $\begin{array}{l}\text { Abnormal } \\
\text { movements }\end{array}$ & 17 & 10 & 1.923 & 0.166 \\
\hline Agitated / restless & 19 & 7 & 5.856 & 0.016 \\
\hline Loss of appetite & 9 & 14 & 1.142 & 0.285 \\
\hline Weight loss & 13 & 10 & 0.411 & 0.521 \\
\hline Other misc effects & 12 & 9 & 0.448 & 0.503 \\
\hline $\begin{array}{l}\text { Dizzy / lightheaded / } \\
\text { faint }\end{array}$ & 12 & 10 & 0.191 & 0.662 \\
\hline Diarrhea / gas & 14 & 8 & 1.715 & 0.19 \\
\hline $\begin{array}{l}\text { Dry mouth / sore } \\
\text { throat }\end{array}$ & 10 & 10 & 0.000 & 1.00 \\
\hline $\begin{array}{l}\text { Increase appetite / } \\
\text { cravings }\end{array}$ & 12 & 8 & 0.835 & 0.361 \\
\hline $\begin{array}{l}\text { Ability to do things } \\
\text { in absence of } \\
\text { symptoms }\end{array}$ & 6 & 13 & 2.685 & 0.101 \\
\hline Yawning & 12 & 3 & -- & -- \\
\hline Other sexual effects & 8 & 6 & 0.294 & 0.587 \\
\hline
\end{tabular}

${ }^{\mathrm{a}} \mathrm{p}$-value set at 0.001 for $\mathrm{k}=35$ comparisons in Tables 4.14 and 4.15 


\section{Seroquel.}

Table 4.16 shows the percentage of consumers mentioning an effect of Seroquel grouped by the category of effect (also see Appendix D). Sleep effects were mentioned by over $60 \%$ of consumers, followed by nearly $52 \%$ of consumers mentioning mental or mood effects.

Table 4.17 lists all effects of Seroquel mentioned by at least 3\% of consumers. The top 2 effects were related to sleep, including the drug helps sleep or causes too much sleep and tiredness. About a quarter of consumers mentioned an improvement in symptoms, such as anxiety, mania, or hallucinations, and about $10 \%$ mentioned these same symptoms newly appearing or worsening. Weight gain was mentioned by $22.5 \%$ of consumers, and increased appetite and cravings (especially sugar and carbohydrate cravings) were mentioned by $12.7 \%$ of consumers.

Figure 4.2 and Table 4.18 show the most commonly reported effects for Seroquel across 4 websites. The bar chart in Figure 4.2 illustrates greater homogeneity in reported effects across websites as compared to the same chart in Figure 4.1 for Lexapro. With pvalues set at 0.002 for multiple comparisons involving the originating websites, statistically significant chi-square coefficients were reached for 2 of the 8 effects listed: extreme sleepiness and weight gain. Consumers on AskaPatient mentioned these effects at a considerably higher rate than the other 3 websites. Consumers on RevolutionHealth mentioned symptom improvement more often than consumers on other websites, and mentioned new or worsening symptoms the least often. WebMD also had fewer reports of new or worsening symptoms compared to the 2 consumer-constructed websites, and had the least mentions of all websites of abnormal movements. 
In Table 4.19, effects listed by at least $3 \%$ of consumers are compared across consumer- and expert-constructed websites. One of the 18 effects listed showed significant values at the $\mathrm{p}<0.002$ level, indicating that there was an association between new/worsened symptoms and the classification of the website on which it was reported. Significantly more consumers on consumer-constructed websites reported Seroquel worsened or induced new symptoms $(\mathrm{p}=.000)$, while more consumers on expertconstructed sites reported it improved their symptoms ( $\mathrm{p}=.008)$. Similarly, a higher percentage of consumers posting on consumer-constructed sites reported the drug made them excessively sleepy and tired $(\mathrm{p}=.009)$, while those on expert-constructed websites reported that it helps with sleep $(\mathrm{p}=.105)$.

In sum, effects on mental or mood symptoms and effects on sleep were reported by consumers for both drugs more often than any other types of effects. In general, reporting across websites was more heterogeneous for Lexapro than for Seroquel. Consumers across each website and for both drugs reported improvements in mental or mood symptoms at a higher rate than worsened or induced symptoms. However, the trend for both drugs was that consumers on expert-constructed sites reported higher rates of symptom improvement (and helpful sleep on Seroquel), but generally lower rates of side effects. 
Table 4.16

Top 5 Effect Categories Mentioned by Consumers for Seroquel

\begin{tabular}{|r|c|}
\hline \multicolumn{1}{|c|}{ Category of effects } & \% of consumers mentioning the effect \\
\hline Sleep effects & $60.6 \%$ \\
\hline Mental or mood effects & $52.9 \%$ \\
\hline Appetite and weight effects & $30.8 \%$ \\
\hline Musculoskeletal and neurological effects & $18.1 \%$ \\
\hline Other effects & $16.0 \%$ \\
\hline
\end{tabular}


Table 4.17

Effects of Seroquel Mentioned by at Least 3\% of Consumers

\begin{tabular}{|c|c|}
\hline Drug effect & $\begin{array}{l}\% \text { of consumers mentioning the effect } \\
\qquad(\mathrm{N}=480)\end{array}$ \\
\hline Helps me sleep & $35.6 \%$ \\
\hline Extreme sleepiness / tired & $33.1 \%$ \\
\hline Symptoms reduced / improved & $24.8 \%$ \\
\hline Weight gain & $22.5 \%$ \\
\hline Brain fog / zombie & $15.2 \%$ \\
\hline Increased appetite / cravings & $12.7 \%$ \\
\hline Symptoms new / worsened & $10.2 \%$ \\
\hline Abnormal movements & $8.8 \%$ \\
\hline Emotionally stable / calm / "normal" & $7.9 \%$ \\
\hline Difficult to wake up & $6.3 \%$ \\
\hline Other misc effects & $5.0 \%$ \\
\hline Dizzy / lightheaded / faint & $4.8 \%$ \\
\hline Blood sugar high / diabetes & $4.8 \%$ \\
\hline Dry mouth / sore throat & $4.6 \%$ \\
\hline Dreams vivid / nightmares & $4.4 \%$ \\
\hline $\begin{array}{l}\text { Numb / detached / lack of energy or } \\
\text { interest }\end{array}$ & $4.2 \%$ \\
\hline Memory loss & $3.8 \%$ \\
\hline Hungover / drunk & $3.1 \%$ \\
\hline
\end{tabular}


Figure 4.2 Most Popularly Mentioned Seroquel Effects by Consumers across Websites

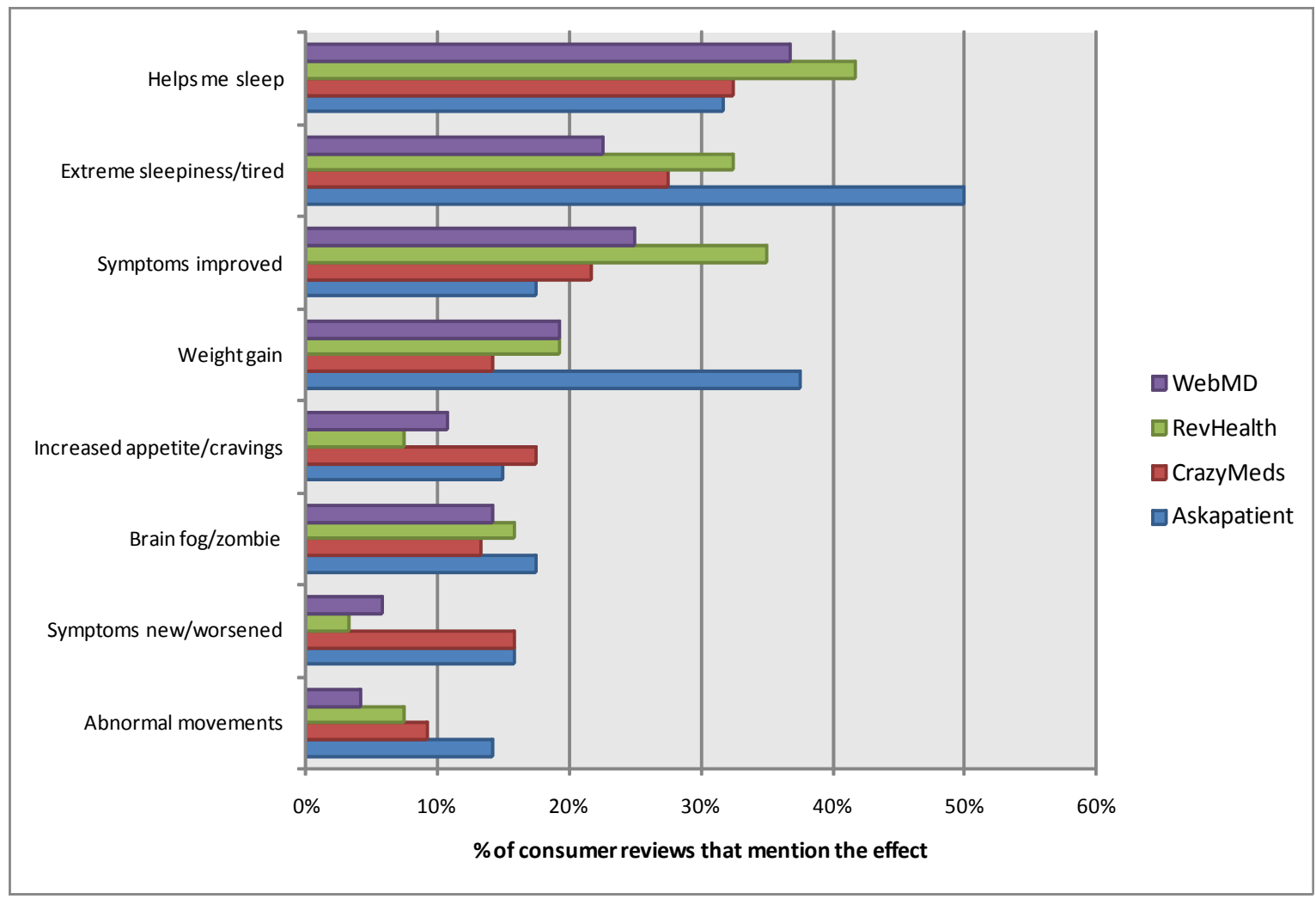


Table 4.18

Number of Consumers on Each Website Mentioning the Most Popularly Reported Effects of Seroquel ${ }^{a}$

\begin{tabular}{|l|c|c|c|c|c|c|}
\hline & $\begin{array}{c}\text { AskaPatient } \\
\mathrm{N}=120\end{array}$ & $\begin{array}{c}\text { CrazyMeds } \\
\mathrm{N}=120\end{array}$ & $\begin{array}{c}\text { RevHealth } \\
\mathrm{N}=120\end{array}$ & $\begin{array}{c}\text { WebMD } \\
\mathrm{N}=120\end{array}$ & $\begin{array}{c}\text { Chi- } \\
\text { square }\end{array}$ & $\begin{array}{c}\mathrm{p}- \\
\text { value }\end{array}$ \\
\hline Helps me sleep & 38 & 39 & 50 & 44 & 3.298 & 0.348 \\
\hline $\begin{array}{l}\text { Extreme } \\
\text { sleepiness/ tired }\end{array}$ & 60 & 33 & 39 & 27 & 23.276 & 0.000 \\
\hline $\begin{array}{l}\text { Symptoms } \\
\text { improved }\end{array}$ & 21 & 26 & 42 & 30 & 10.76 & 0.013 \\
\hline $\begin{array}{l}\text { Weight gain } \\
\text { Increase appetite/ } \\
\text { cravings }\end{array}$ & 18 & 21 & 9 & 13 & 6.366 & 0.095 \\
\hline $\begin{array}{l}\text { Brain fog/zombie } \\
17\end{array}$ & 21 & 16 & 19 & 17 & 0.953 & 0.813 \\
\hline $\begin{array}{l}\text { Symptoms new/ } \\
\text { worsened }\end{array}$ & 19 & 9 & 5 & 7.828 & 0.05 \\
\hline $\begin{array}{l}\text { Abnormal } \\
\text { movements }\end{array}$ & 19 & 4 & 7 & --000 & - \\
\hline
\end{tabular}

${ }^{a} \mathrm{p}$-value set at 0.002 for $\mathrm{k}=25$ comparisons in Tables 4.18 and 4.19 
Table 4.19

Seroquel Effects Mentioned by $>3 \%$ of Consumers According to Website Classification

\begin{tabular}{|c|c|c|c|c|}
\hline Drug effect & $\begin{array}{l}\begin{array}{l}\text { Consumer- } \\
\text { constructed }\end{array} \\
(\mathrm{N}=220)\end{array}$ & $\begin{array}{c}\text { Expert- } \\
\text { constructed } \\
(\mathrm{N}=220)\end{array}$ & Chi-square & p-value \\
\hline Helps me sleep & 77 & 94 & 2.625 & 0.105 \\
\hline $\begin{array}{l}\text { Extreme sleepiness / } \\
\text { tired }\end{array}$ & 93 & 66 & 6.856 & 0.009 \\
\hline $\begin{array}{l}\text { Symptoms reduced / } \\
\text { improved }\end{array}$ & 47 & 72 & 6.983 & 0.008 \\
\hline Weight gain & 62 & 46 & 3.059 & 0.08 \\
\hline Brain fog / zombie & 37 & 36 & 0.016 & 0.899 \\
\hline $\begin{array}{l}\text { Increased appetite / } \\
\text { cravings }\end{array}$ & 39 & 22 & 5.427 & 0.02 \\
\hline $\begin{array}{l}\text { Symptoms new / } \\
\text { worsened }\end{array}$ & 38 & 11 & 16.569 & 0.000 \\
\hline $\begin{array}{l}\text { Abnormal } \\
\text { movements }\end{array}$ & 28 & 14 & 5.114 & 0.024 \\
\hline $\begin{array}{l}\text { Emotionally stable / } \\
\text { calm / "normal" }\end{array}$ & 25 & 13 & 4.115 & 0.042 \\
\hline Difficult to wake up & 19 & 11 & 2.276 & 0.131 \\
\hline Other misc effects & 13 & 11 & 0.175 & 0.675 \\
\hline $\begin{array}{l}\text { Dizzy / lightheaded / } \\
\text { faint }\end{array}$ & 11 & 12 & 0.046 & 0.831 \\
\hline $\begin{array}{l}\text { Blood sugar high / } \\
\text { diabetes }\end{array}$ & 13 & 10 & 0.411 & 0.521 \\
\hline Dry mouth / sore & 15 & 7 & 3.049 & 0.081 \\
\hline
\end{tabular}




\begin{tabular}{|l|c|c|c|c|}
\hline \multicolumn{1}{|c|}{ Drug effect } & $\begin{array}{c}\text { Consumer- } \\
\text { constructed } \\
(\mathrm{N}=220)\end{array}$ & $\begin{array}{c}\text { Expert- } \\
\text { constructed } \\
(\mathrm{N}=220)\end{array}$ & Chi-square & p-value \\
\hline throat & 16 & 5 & 6.026 & 0.014 \\
\hline $\begin{array}{l}\text { Dreams vivid / } \\
\text { nightmares }\end{array}$ & 13 & 7 & 1.878 & 0.171 \\
\hline $\begin{array}{l}\text { Numb / detached / } \\
\text { lack of energy or } \\
\text { interest }\end{array}$ & 11 & 7 & 0.924 & 0.337 \\
\hline Memory loss & 10 & 5 & 1.720 & 0.19 \\
\hline Hungover / drunk & & & & \\
\hline
\end{tabular}

${ }^{\mathrm{a}} \mathrm{p}$-value set at 0.002 for $\mathrm{k}=25$ comparisons in Tables 4.18 and 4.19

\section{Drugs Effects According to Experts}

\section{Lexapro.}

Table 4.20 lists a count and proportion of the most frequently mentioned effects of Lexapro in expert text. For example, abnormal movements were mentioned 1 time on RevolutionHealth and mentioned 4 times on WebMD. As a proportion of the total frequency of mentions of all effects, abnormal movements made up $2.6 \%$ and $3.2 \%$ of all mentions of effects on RevolutionHealth and WebMD, respectively. In all but two instances (agitated/restless and brain fog/zombie), WebMD cited effects more often than RevolutionHealth. However for several effects, RevolutionHealth showed a higher proportion of the frequency of mentions. For example, WebMD mentioned new or worsening symptoms twice as often as RevolutionHealth ( 8 times versus 4 times). Out of all mentions of effects on RevolutionHealth, though, $10.3 \%$ were related to new or 
worsening symptoms, whereas $6.5 \%$ of total mentions on WebMD were this effect. Figures 4.3 and 4.4 illustrate this difference using the top 6 most frequently mentioned effects in expert text as measured by number of mentions (Figure 4.3) and proportion of all mentions (Figure 4.4). Four of the top 6 effects were the most frequently mentioned regardless of measurement: blood related changes, suicidal thinking/planning, symptoms new/worsened, and other misc effects. The remaining 2 effects changed depending on whether the measurement was number or proportion of mentions. In both cases, WebMD had the highest number of mentions (nausea/vomiting and dizzy/lightheaded/faint), whereas RevolutionHealth had the highest proportion of mentions (brain fog/zombie and agitated/restless).

The most frequently mentioned effect in WebMD fell under the code "other misc effects," which included effects that were sparsely reported across websites, such as serotonin syndrome, toothache, kidney failure, and bronchitis. The most frequently mentioned effects in RevolutionHealth were agitated/restless, suicidal thinking/planning, and symptoms new/worsened, each appearing 4 times in that text. Additionally, 4 of the 14 effects listed in Table 4.20 were mentioned in one expert text and not the other. In only 1 of these instances did RevolutionHealth cite an effect (brain fog/zombie) not mentioned on WebMD. 
Table 4.20

Most Frequently Mentioned Effects of Lexapro in Expert Text (frequency $>3 \%$ of total mentions of effects on either website)

\begin{tabular}{|c|c|c|}
\hline & \multicolumn{2}{|c|}{$\begin{array}{l}\text { \# of times effect is mentioned in text } \\
(\% \text { of all mentions of effects in text) }\end{array}$} \\
\hline & RevolutionHealth & WebMD \\
\hline Other misc effects & $1(2.6 \%)$ & $13(10.5 \%)$ \\
\hline Agitated/restless & $4(10.3 \%)$ & $3(2.4 \%)$ \\
\hline $\begin{array}{r}\text { Suicidal thinking/ } \\
\text { planning }\end{array}$ & $4(10.3 \%)$ & $5(4.0 \%)$ \\
\hline $\begin{array}{r}\text { Symptoms new/ } \\
\text { worsened }\end{array}$ & $4(10.3 \%)$ & $8(6.5 \%)$ \\
\hline Brain fog/zombie & $3(7.7 \%)$ & $0(0 \%)$ \\
\hline Blood related changes & $0(0 \%)$ & $9(7.3 \%)$ \\
\hline Dizzy/lightheaded/ faint & $2(5.1 \%)$ & $7(5.6 \%)$ \\
\hline Extreme sleepiness/tired & $2(5.1 \%)$ & $4(3.2 \%)$ \\
\hline $\begin{array}{r}\text { Trouble sleeping/ } \\
\text { insomnia }\end{array}$ & $2(5.1 \%)$ & $3(2.4 \%)$ \\
\hline $\begin{array}{r}\text { Nausea/sick } \\
\text { stomach/vomiting }\end{array}$ & $1(2.6 \%)$ & $6(4.8 \%)$ \\
\hline Heartbeat changes & $1(2.6 \%)$ & $4(3.2 \%)$ \\
\hline Abnormal movements & $1(2.6 \%)$ & $4(3.2 \%)$ \\
\hline Fever/chills/infection & $0(0 \%)$ & $4(3.2 \%)$ \\
\hline Other sexual effects & $0(0 \%)$ & $4(3.2 \%)$ \\
\hline
\end{tabular}


Figure 4.3 Top 6 Most Frequently Mentioned Lexapro Effects in Expert Text Measured by Number of Mentions

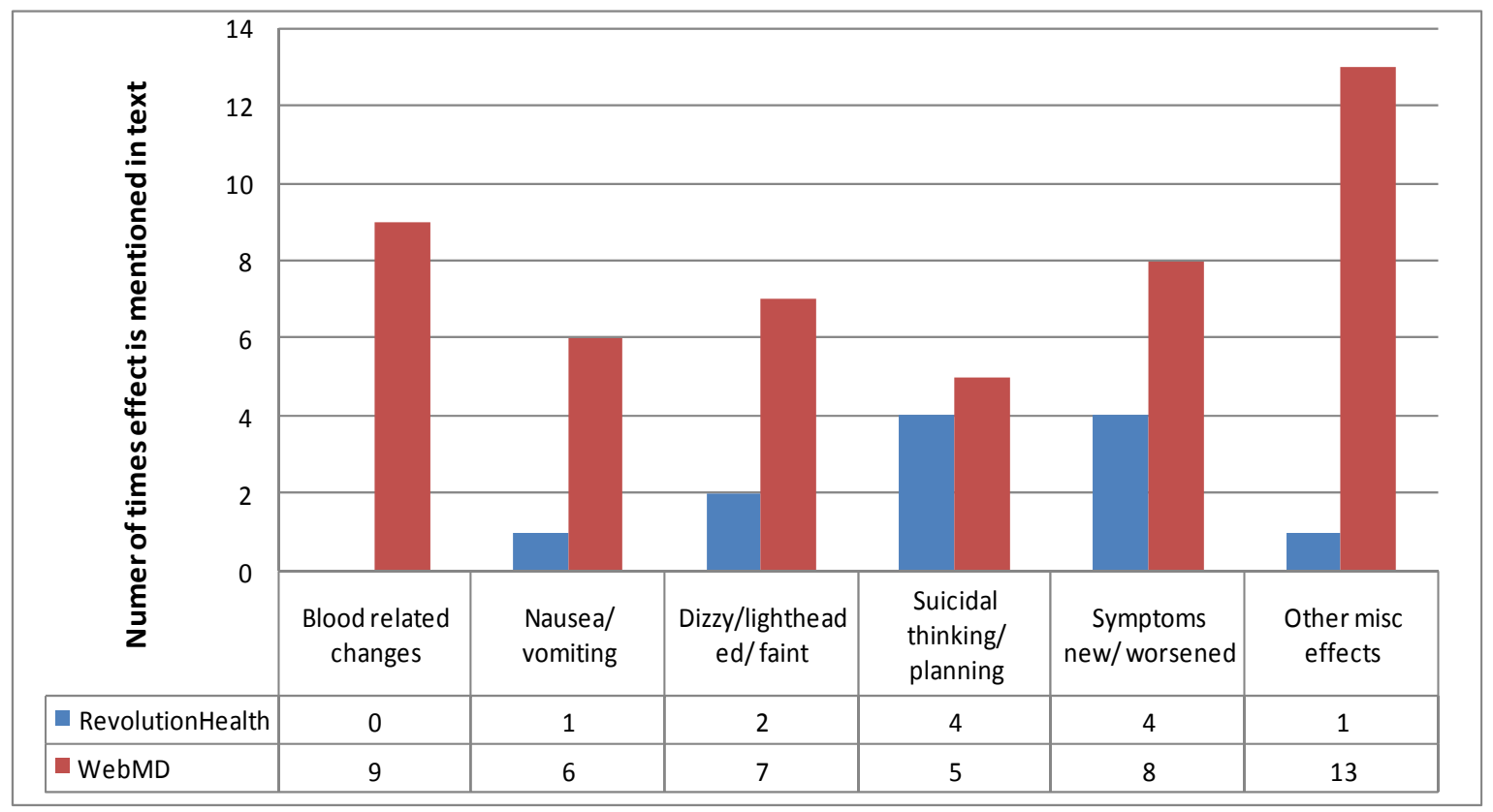


Figure 4.4 Top 6 Most Frequently Mentioned Lexapro Effects in Expert Text Measured by Proportion of All Mentions of Effects

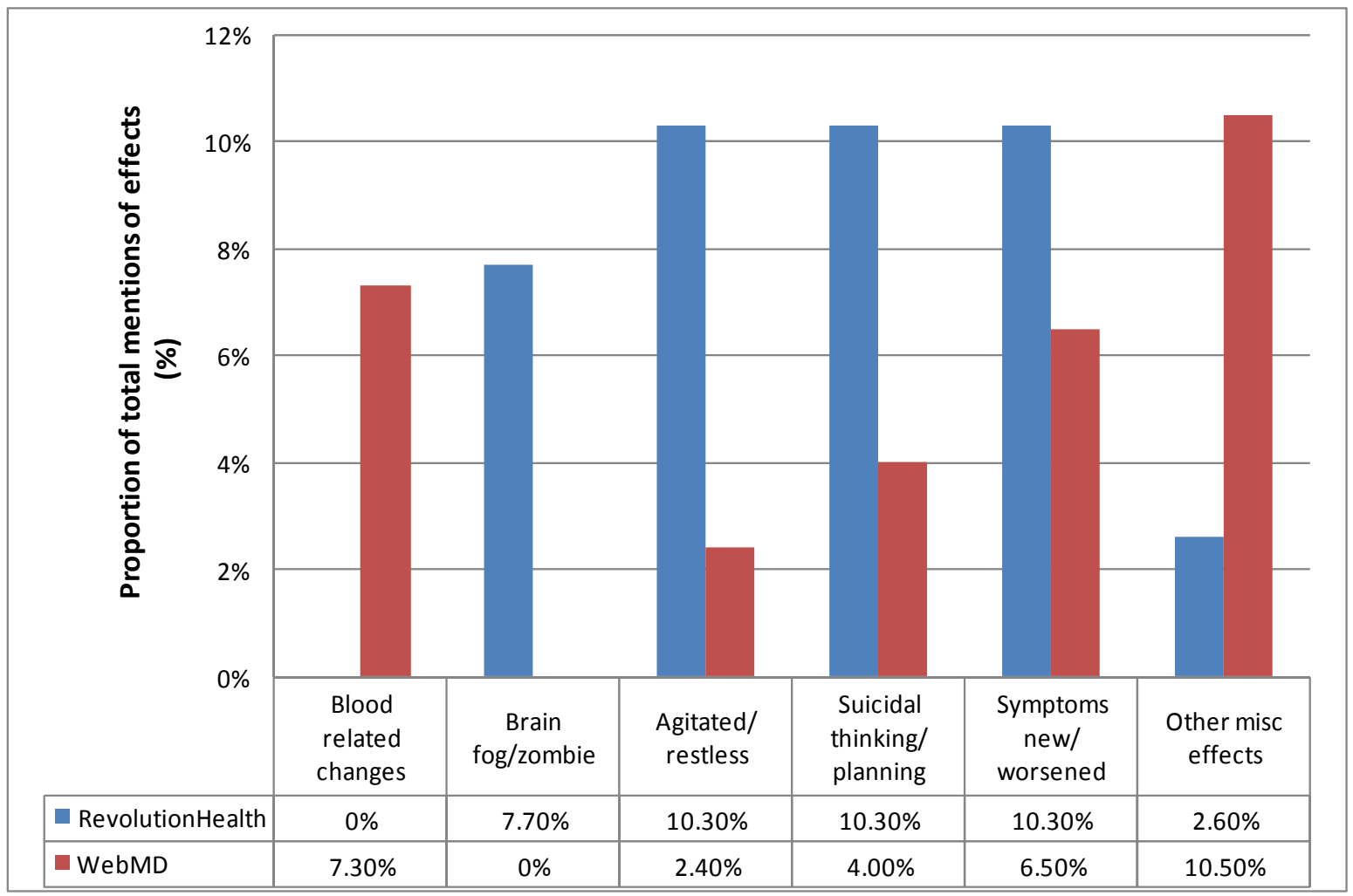

\section{Seroquel.}

Table 4.21 lists a count and proportion of the most frequently mentioned effects of Seroquel in expert text. RevolutionHealth mentioned 11 effects more frequently than WebMD, with 5 of these related to mental or mood changes. WebMD mentioned 8 effects more frequently than RevolutionHealth, all of which were physical effects. Figures 4.5 and 4.6 illustrate that the proportion of total mentions of effects remained consistent with the number of mentions on each website. For example, RevolutionHealth mentioned suicidal thinking/planning and symptoms new/worsened more frequently than WebMD as measured both by the number of mentions and proportion of all mentions. 
The most frequently mentioned effect in WebMD was dizzy/lightheaded/faint (7 mentions). In RevolutionHealth, they were brain fog/zombie and symptoms new/worsened (5 mentions each). For 3 of the 21 effects listed in Table 4.21, an effect was mentioned on one expert website and not the other. Increased energy/euphoria/mania and increased appetite/cravings were mentioned 2 times and 3 times, respectively, on RevolutionHealth and zero times on WebMD. Other labs and chronic conditions, such as pancreatitis and underactive thyroid, were mentioned 4 times on WebMD and not at all on RevolutionHealth.

For both drugs, it appeared that the expert text on WebMD more frequently mentioned physical effects, such as blood-related changes and nausea/vomiting, while RevolutionHealth more frequently mentioned mental or mood "side effects," such as brain fog/zombie and agitated/restless. Most of the commonly reported effects in Tables 4.20 and 4.21 were cited at least once in both texts, however for both drugs there were considerable discrepancies between websites in the relative attention (measured by number and proportion of mentions) given to effects. 
Table 4.21

Most Frequently Mentioned Effects of Seroquel in Expert Text (frequency $>3 \%$ of total mentions of effects in either website)

\begin{tabular}{|c|c|c|}
\hline & \multicolumn{2}{|c|}{$\begin{array}{l}\text { \# of times effect is mentioned in text } \\
(\% \text { of all mentions of effects in text) }\end{array}$} \\
\hline & RevolutionHealth & WebMD \\
\hline Brain fog/zombie & $5(7.6 \%)$ & $1(1.0 \%)$ \\
\hline Symptoms new/worsened & $5(7.6 \%)$ & $1(1.0 \%)$ \\
\hline Dizzy/lightheaded/faint & $2(3.0 \%)$ & $7(6.9 \%)$ \\
\hline Suicidal thinking/ planning & $4(6.1 \%)$ & $2(2.0 \%)$ \\
\hline Weakness & $4(6.1 \%)$ & $2(2.0 \%)$ \\
\hline Abnormal movements & $2(3.0 \%)$ & $5(4.9 \%)$ \\
\hline Vision/eye changes & $3(4.5 \%)$ & $3(2.9 \%)$ \\
\hline Headache increased & $3(4.5 \%)$ & $2(2.0 \%)$ \\
\hline Agitated/restless & $3(4.5 \%)$ & $1(1.0 \%)$ \\
\hline $\begin{array}{r}\text { Heaviness/soreness/ } \\
\text { pain/numbness }\end{array}$ & $3(4.5 \%)$ & $1(1.0 \%)$ \\
\hline Urinary changes/ problems & $3(4.5 \%)$ & $1(1.0 \%)$ \\
\hline Increased appetite/ cravings & $3(4.5 \%)$ & $0(0 \%)$ \\
\hline Extreme sleepiness/ tired & $2(3.0 \%)$ & $4(3.9 \%)$ \\
\hline Other misc effects & $2(3.0 \%)$ & $4(3.9 \%)$ \\
\hline Blood sugar high/ diabetes & $1(1.5 \%)$ & $4(3.9 \%)$ \\
\hline $\begin{array}{r}\text { Other labs or chronic } \\
\text { conditions }\end{array}$ & $0(0 \%)$ & $4(3.9 \%)$ \\
\hline Fever/chills/infection & $2(3.0 \%)$ & $3(2.9 \%)$ \\
\hline
\end{tabular}




\begin{tabular}{|r|c|c|}
\hline & \multicolumn{2}{|c|}{$\begin{array}{c}\text { \# of times effect is mentioned in text } \\
\text { (\% of all mentions of effects in text) }\end{array}$} \\
\hline Heartbeat changes & RevolutionHealth & WebMD \\
\hline "Sweating like a pig" & $2(3.0 \%)$ & $2(2.0 \%)$ \\
\hline Trouble sleeping/ insomnia & $2(3.0 \%)$ & $1(1.0 \%)$ \\
\hline $\begin{array}{r}\text { Energy increased/ } \\
\text { euphoria/mania }\end{array}$ & $2(3.0 \%)$ & $0(0 \%)$ \\
\hline
\end{tabular}

Figure 4.5 Top 6 Most Frequently Mentioned Seroquel Effects in Expert Text Measured by Number of Mentions

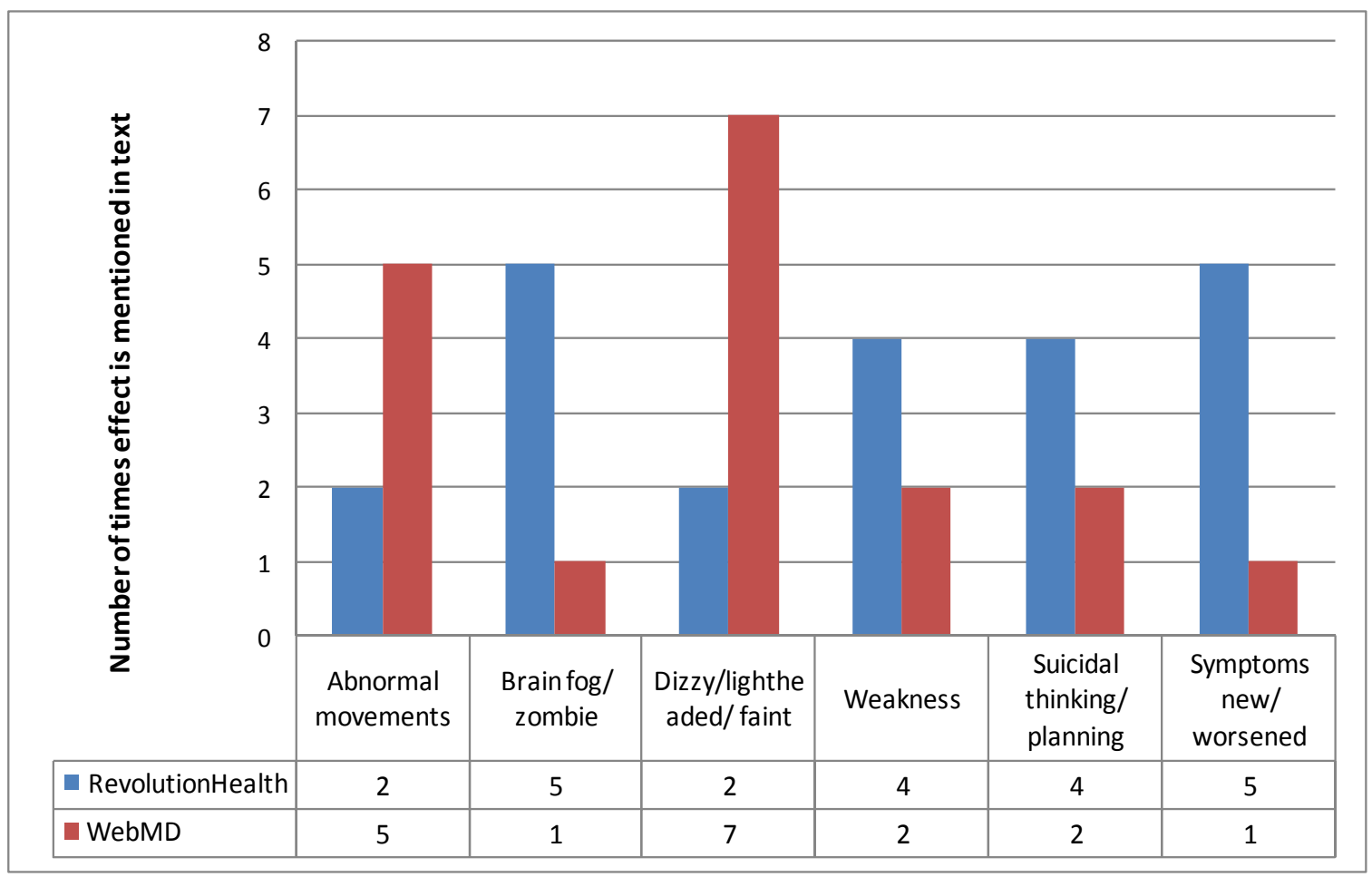


Figure 4.6 Top 6 Most Frequently Mentioned Seroquel Effects in Expert Text Measured by Proportion of All Mentioned of Effects

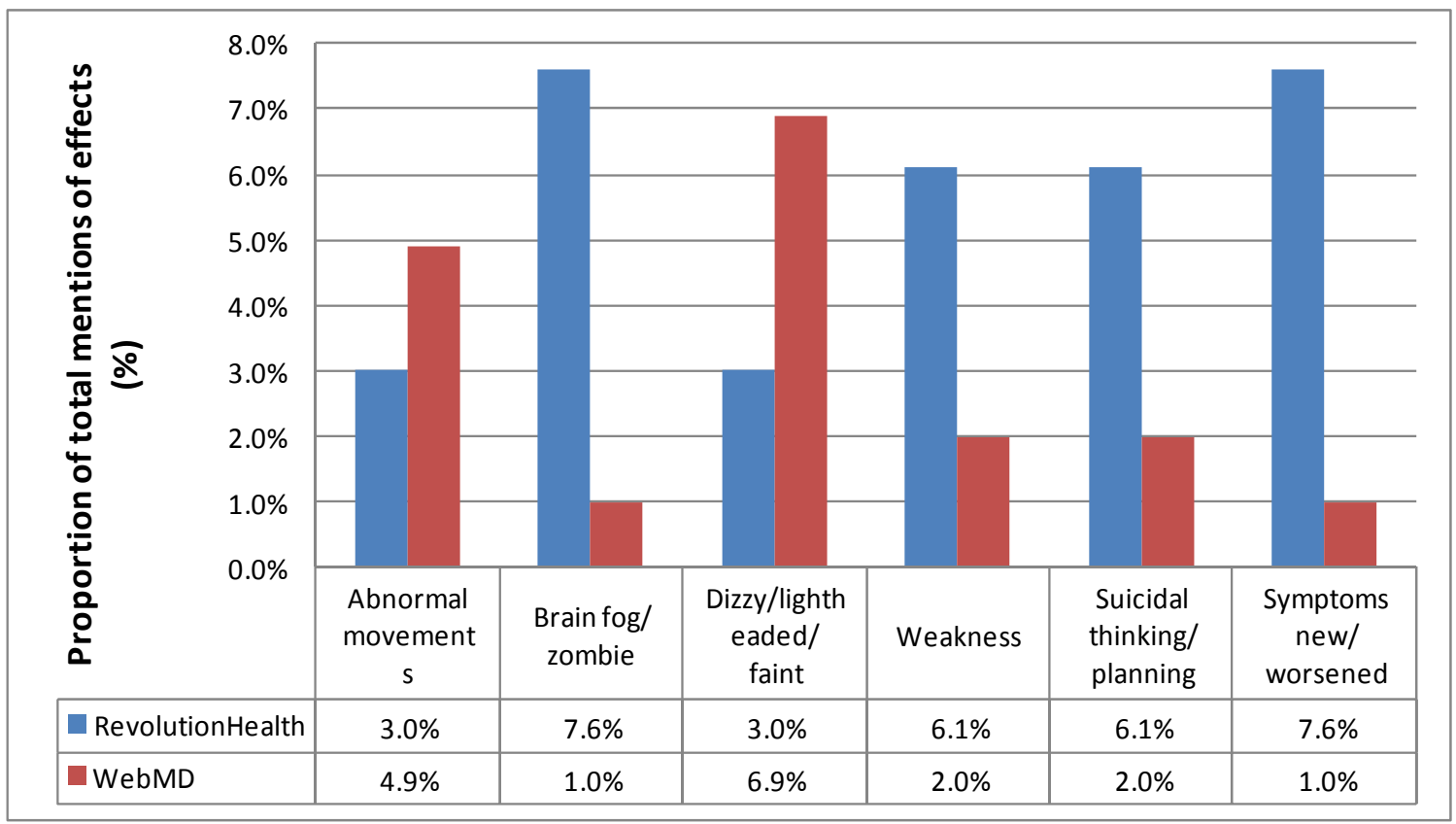

Drug Effects by Experts versus Consumers

\section{Lexapro.}

Experts and consumers both mentioned the category mental or mood effects more frequently than any other category of effects (Figure 4.7). There was considerable discrepancy between experts and consumers for musculoskeletal/neurological effects, mentioned more often by experts, and sleep effects, mentioned more often by consumers.

Figure 4.8 compares experts and consumers on the most frequently mentioned individual effects from both groups. Consumers gave considerably more attention than experts to symptom improvement, extreme sleepiness, and weight gain. New or worsening symptoms was one of the most frequently mentioned effects by consumers (Table 4.13), but mentions of this effect actually made up a larger proportion of total 
mentions in the expert text (Figure 4.8). Experts gave the most attention to other misc effects, blood related changes, suicidal thinking/planning, and dizzy/lightheaded/faint, while consumers mentioned these effects much less frequently.

As seen in Figure 4.8, experts and consumers did have comparable rates of mentions for new or worsening symptoms (7.4\% and $6.5 \%$ of mentions, respectively) and nausea/sick stomach/vomiting ( $4.3 \%$ and $4.1 \%$ of mentions, respectively). Table 4.22 provides a representative selection of excerpts from each website of text coded “symptoms new/worsened," with some overlap of suicidal thinking/planning as, when mentioned, the latter effect often co-occured with the former. Experts provided a laundry list of new or worsening symptoms for users to beware of. As the excerpts in Table 4.22 demonstrate, the expert list of symptoms and consumers' reports of new/worsening symptoms appeared to largely overlap. Reports from consumers, however, provided situational examples and context for how the effect may be experienced or manifested in a single individual.

Tables 4.23 and 4.24 compare experts and consumers on frequency of mentions and descriptions of weight and appetite effects and sexual effects, respectively. These categories were selected to compare and contrast expert and consumer descriptions because of their richness of data. Table 4.23 shows that out of all mentions of effects within the category weight and appetite effects, experts gave the most attention to loss of appetite, while consumers gave the most attention to weight gain. WebMD described increased appetite as rare and decreased appetite as infrequent. An almost even number of consumers (4.2\% and 4.8\%, respectively) mentioned each of these effects at least once, though decreased appetite was mentioned more frequently as a proportion of all mentions 
of weight and appetite effects. Additionally, experts described each effect on weight and appetite to be less serious or less severe, while consumers demonstrated more clearly differentiated perspectives such as "hugest problem of all" and "appalling" in relation to weight gain, and "an extra bonus" in relation to weight loss.

Table 4.24 summarizes the same information for sexual effects of Lexapro. Most of the mentions of sexual effects in expert text were related to other sexual effects, such as the nondescript "sexual problems" or priapism (all from WebMD). Consumers gave more attention to lost sex drive ( $42.2 \%$ of mentions) and trouble achieving orgasm (37\% of mentions), and much less attention to other sexual effects (11.9\% of mentions). Lost sex drive was mentioned by $10.6 \%$ of consumers, though WebMD described this effect as infrequent and RevolutionHealth did not cite this effect at all. At the same time, trouble achieving orgasm was mentioned by slightly fewer consumers (8.5\%), but was labeled a frequent effect by WebMD. Trouble achieving orgasm and other sexual effects were described as less serious or less severe by experts, while lost sex drive was rated as severe by WebMD (worded as "decreased interest in sex"). Consumers described all sexual effects using terms such as "the absolute worst, "extremely frustrating," and similar. Some consumers made comments like, "I want to quit... so I can have a frigging orgasm," or "can't perform sexually so you get depressed and anxious." 
Figure 4.7 Comparison of Frequency of Mentions of Lexapro Effects between Experts and Consumers, by Effect Category

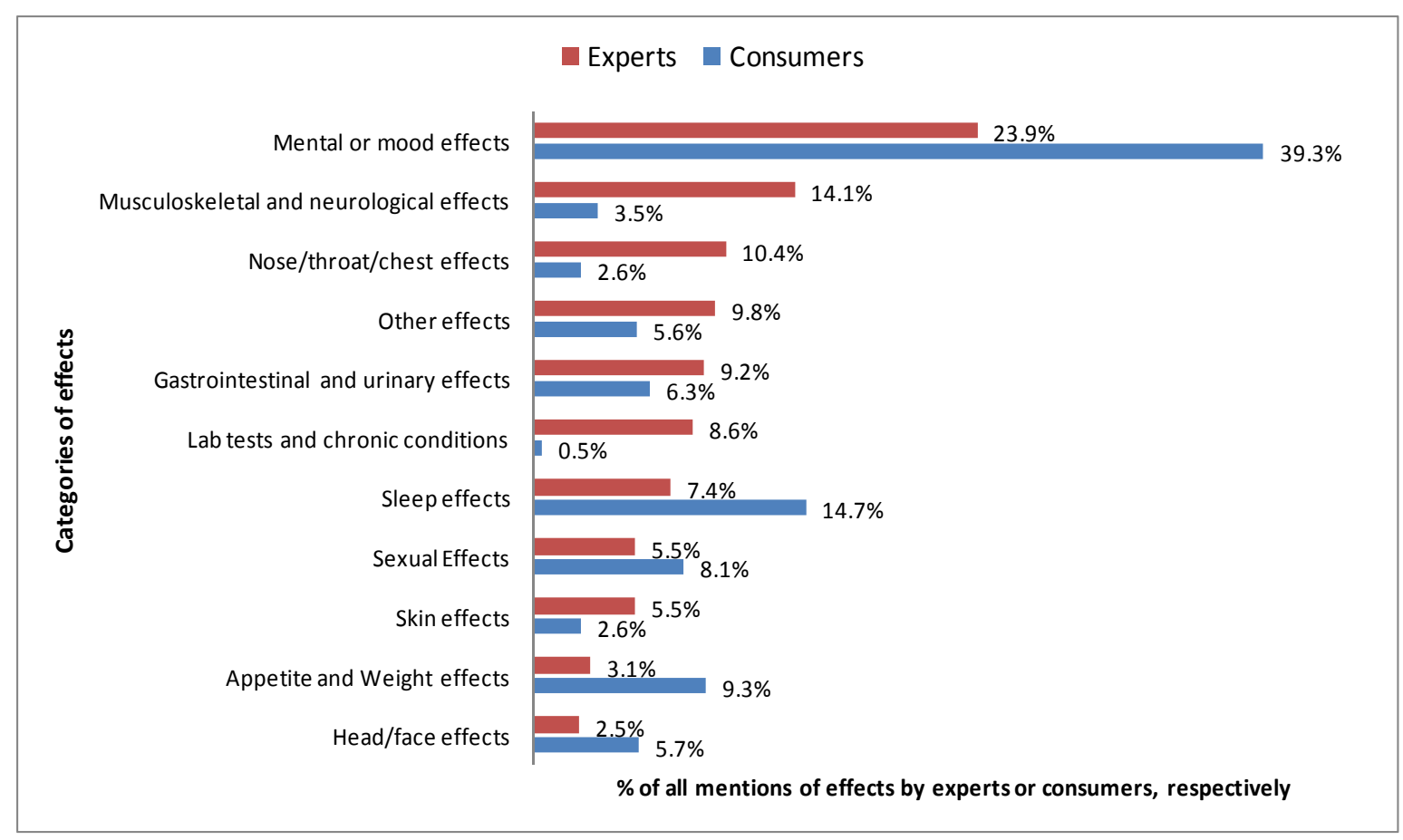


Figure 4.8 Comparison of Most Frequently Mentioned Lexapro Effects between Experts and Consumers

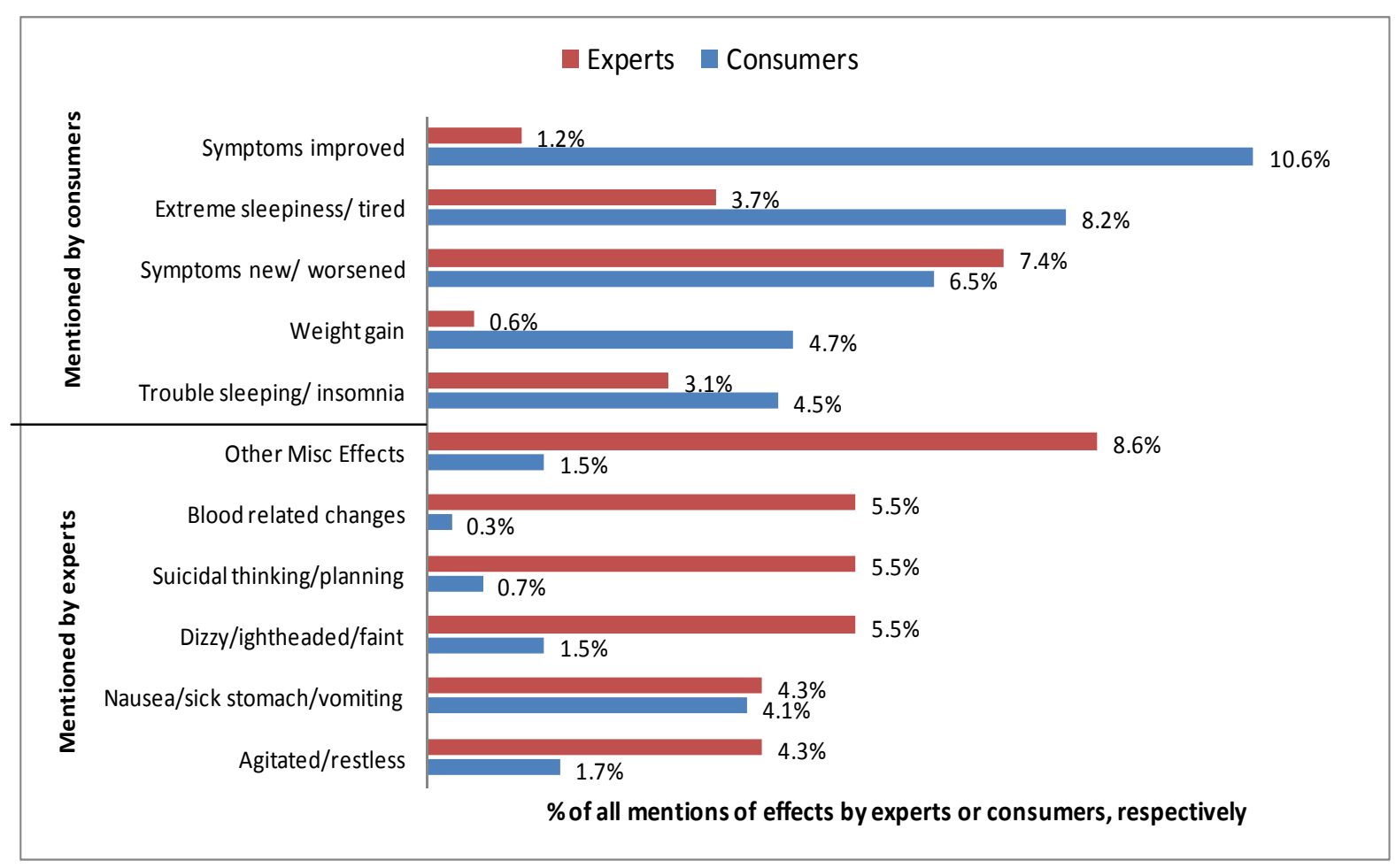


Table 4.22

Excerpts from Experts and Consumers on Selected Mental/Mood Effects of Lexapro

\begin{tabular}{|c|c|}
\hline \multicolumn{2}{|r|}{ Effects: Symptoms new/worsened; Suicidal thinking/planning } \\
\hline WebMD (Expert) & $\begin{array}{l}\text { "Tell the doctor immediately if you notice worsening } \\
\text { depression/other psychiatric conditions, unusual behavior changes } \\
\text { (including possible suicidal thoughts/attempts), or other mental/mood } \\
\text { changes (including new/worsening anxiety, panic attacks, trouble } \\
\text { sleeping, irritability, hostile/angry feelings, impulsive actions, severe } \\
\text { restlessness, very rapid speech)" } \\
\text { "Tell your doctor immediately if any of these unlikely but serious } \\
\text { side effects occur: unusual or severe mental/mood changes (e.g., } \\
\text { nervousness, unusual high excitement/energy, rare thoughts of } \\
\text { suicide)..." }\end{array}$ \\
\hline $\begin{array}{l}\text { RevHealth } \\
\text { (Expert) }\end{array}$ & $\begin{array}{l}\text { "Call your doctor at once if you have any new or worsening } \\
\text { symptoms such as: mood or behavior changes, anxiety, panic attacks, } \\
\text { trouble sleeping, or if you feel impulsive, irritable, agitated, hostile, } \\
\text { aggressive, restless, hyperactive (mentally or physically), more } \\
\text { depressed, or have thoughts about suicide or hurting yourself" }\end{array}$ \\
\hline AskaPatient & $\begin{array}{l}\text { "And then the worst crippling panic attacks I have ever had to date" } \\
(\# 8) \\
\text { "I got even more depressed that I EVER WAS, even before taking } \\
\text { the med" (\#33) } \\
\text { "I seemed to become more aggressive and assertive. I would just } \\
\text { speak my mind whenever I got angry, and had no fear. I seemed to } \\
\text { become more "mean" and "mad" and I just didn't like myself" (\#41) }\end{array}$ \\
\hline CrazyMeds & $\begin{array}{l}\text { "had some hypomania then extreme agitation then suicidality. The } \\
\text { agitation was awful, felt like I was going to jump out of my skin - } \\
\text { and my mind was racing." (\#172) } \\
\text { "2 hours of alternating panic attacks/crying jags" (\#130) }\end{array}$ \\
\hline $\begin{array}{l}\text { RevHealth } \\
\text { (Consumers) }\end{array}$ & $\begin{array}{l}\text { "A couple of days later I had my first manic experience which lasted } \\
\text { about } 30 \text { minutes of complete reckless driving, I probably should } \\
\text { have gotten arrested. And a few minutes later I came down into deep } \\
\text { depression" (\#258) }\end{array}$ \\
\hline
\end{tabular}




\begin{tabular}{|l|l|}
\hline $\begin{array}{l}\text { WebMD } \\
\text { Consumers) }\end{array}$ & $\begin{array}{l}\text { "I have been very hostile and irritable on this med and my panic } \\
\text { attacks have been coming more often and they have been much } \\
\text { worse! I have no patience with my kids or my fiancé, or basically } \\
\text { anyone around me." (\#364) }\end{array}$ \\
\hline
\end{tabular}

Table 4.23

Comparison of Experts and Consumers on "Appetite and Weight Effects" of Lexapro

\begin{tabular}{|c|c|c|c|c|c|c|}
\hline & \multicolumn{2}{|c|}{$\begin{array}{l}\% \text { of mentions out of } \\
\text { all "weight and } \\
\text { appetite effects" }\end{array}$} & \multicolumn{4}{|c|}{ Description of effect } \\
\hline Code & Experts & Consumers & WebMD & $\begin{array}{c}\text { Rev } \\
\text { Health }\end{array}$ & \multicolumn{2}{|c|}{$\begin{array}{l}\% \text { of consumers } \\
\text { mentioning effect; } \\
\text { description of effect }\end{array}$} \\
\hline $\begin{array}{l}\text { Increased } \\
\text { appetite/ } \\
\text { cravings }\end{array}$ & $20 \%$ & $16.8 \%$ & $\begin{array}{l}\text { "rare" } \\
\& \text { "less } \\
\text { severe" }\end{array}$ & -- & $4.2 \%$ & \multirow{2}{*}{$\begin{array}{l}\text { absolutely } \\
\text { ravenous; } \\
\text { out of } \\
\text { control; } \\
\text { hugest } \\
\text { problem of } \\
\text { all; } \\
\text { no relief } \\
\text { even with } \\
\text { exercise }\end{array}$} \\
\hline $\begin{array}{c}\text { Weight } \\
\text { gain }\end{array}$ & $20 \%$ & $50.3 \%$ & -- & $\begin{array}{l}\text { "less } \\
\text { serious" } \\
\text { (weight } \\
\text { changes) }\end{array}$ & $13.1 \%$ & \\
\hline $\begin{array}{l}\text { Loss of } \\
\text { appetite }\end{array}$ & $40 \%$ & $16.1 \%$ & $\begin{array}{l}\text { "common" } \\
\& \text { "less } \\
\text { severe" }\end{array}$ & -- & $4.8 \%$ & \multirow{2}{*}{$\begin{array}{l}\text { better } \\
\text { control over } \\
\text { appetite; } \\
\text { an extra } \\
\text { bonus; } \\
\text { good for } \\
\text { now; } \\
\text { hope it } \\
\text { continues }\end{array}$} \\
\hline $\begin{array}{c}\text { Weight } \\
\text { loss }\end{array}$ & $20 \%$ & $16.8 \%$ & -- & $\begin{array}{l}\text { "less } \\
\text { serious" } \\
\text { (weight } \\
\text { changes) }\end{array}$ & $4.8 \%$ & \\
\hline
\end{tabular}


Table 4.24

Comparison of Experts and Consumers on "Sexual Effects" of Lexapro

\begin{tabular}{|c|c|c|c|c|c|c|}
\hline & \multicolumn{2}{|c|}{$\begin{array}{l}\% \text { of mentions out of } \\
\text { all "sexual effects" }\end{array}$} & \multicolumn{4}{|c|}{ Description of effect } \\
\hline Code & Experts & Consumers & WebMD & Rev & Con & amers \\
\hline $\begin{array}{l}\text { Lost sex } \\
\text { drive }\end{array}$ & $22.2 \%$ & $42.2 \%$ & $\begin{array}{l}\text { "unlikely } \\
\text { but serious" }\end{array}$ & -- & $\begin{array}{l}\text { Mentioned } \\
\text { by } 10.6 \% \\
\text { of } \\
\text { consumers }\end{array}$ & $\begin{array}{l}\text { the absolute } \\
\text { worst; } \\
\text { really suck; }\end{array}$ \\
\hline $\begin{array}{l}\text { Trouble } \\
\text { achieving } \\
\text { orgasm }\end{array}$ & $33.3 \%$ & $37.0 \%$ & $\begin{array}{c}\text { "common" } \\
\& \text { "less } \\
\text { severe" }\end{array}$ & $\begin{array}{c}\text { "less } \\
\text { serious" }\end{array}$ & $\begin{array}{l}\text { Mentioned } \\
\text { by } 8.5 \% \text { of } \\
\text { consumers }\end{array}$ & $\begin{array}{l}\text { very } \\
\text { bothersome; } \\
\text { extremely }\end{array}$ \\
\hline $\begin{array}{l}\text { Other } \\
\text { sexual } \\
\text { effects } \\
\text { (i.e., } \\
\text { "sexual } \\
\text { problems") }\end{array}$ & $44.4 \%$ & $11.9 \%$ & $\begin{array}{c}\text { "infrequent" } \\
\& \text { "less } \\
\text { severe" }\end{array}$ & -- & $\begin{array}{l}\text { Mentioned } \\
\text { by } 3.1 \% \text { of } \\
\text { consumers }\end{array}$ & $\begin{array}{l}\text { frustrating; } \\
\text { bad; } \\
\text { this is crazy }\end{array}$ \\
\hline
\end{tabular}

\section{Seroquel.}

Figure 4.9 compares the frequency of mentions of effects by experts and consumers according to effect categories. Experts and consumers both mentioned effects within the mental or mood category more frequently than effects in any other category, though the rate was considerably higher in the consumer group (39.3\% vs. $23.9 \%)$. The second highest rate of mentions was musculoskeletal/neurological effects by experts, and sleep effects by consumers. Rates of mentions were most similar (though not exactly 
comparable) between the two groups for gastrointestinal/urinary effects, sexual effects, skin effects, and head/face effects.

Figure 4.10 compares experts and consumers on the most frequently mentioned individual effects from both groups. There were large discrepancies for effects related to sleep, symptom improvement, and weight gain, with consumers far more frequently mentioning these effects. The effect "helps me sleep," reflecting the use of Seroquel as a sleep aid, was not at all mentioned in the expert group. There were less extreme discrepancies in the effects most often mentioned by experts, including dizzy/lightheaded/faint, vision/eye changes, weakness, and suicidal thinking/planning.

Experts and consumers had somewhat comparable rates of mentions for three effects: brain fog/zombie (3.6\% and 5.6\% of mentions, respectively), abnormal movements ( $4.2 \%$ and $3.5 \%$ of mentions), and other miscellaneous effects ( $3.6 \%$ and $1.7 \%$ of mentions). The physical effect, abnormal movements, was also similarly described by both groups. Experts listed "jerky muscle movements you cannot control," "involuntary quivering," and "abnormal movements" among other terms, and consumers reported phrases such as "body jerks," "restless legs," "twitches," "tremors," and “involuntary movements." There were, however, differences between experts and consumers in describing the mental/mood effect of brain fog/zombie. Table 4.25 provides a representative selection of excerpts coded "brain fog/zombie" from each website. Most notably, the expert text stated the effect technically, while the consumer text provided examples of the possible impact and consequence of the effect on an individualized level. 
Tables 4.26 and 4.27 compare experts and consumers on the frequency of mentions and descriptions of Seroquel's sleep effects. Consumers' attention was divided evenly between extreme sleepiness/tired and helps me sleep, as measured both by the frequency of mentions out of all sleep effects and the percentage of consumers who reported the effect. Experts most frequently mentioned extreme sleepiness, followed by trouble sleeping/insomnia, and labeled each as less severe/serious. In contrast to WebMD's description of both of these effects as frequent, approximately $33 \%$ of consumers mentioned extreme sleepiness while only $2.3 \%$ of consumers reported trouble sleeping. Additionally, the sample excerpts of selected sleep effects in Table 4.27 show a qualitatively different understanding of these effects between experts and consumers. While experts cited "drowsiness" and "tiredness" as possible effects of Seroquel, consumers reported "coma like sleep," "extreme sleeping," and being "unwakable" for many hours. This same effect was described by consumers as both helpful and unhelpful depending on the context and circumstance of the consumer's life.

Figure 4.9 shows that out of all mentions of effects according to category, $8.6 \%$ of mentions in the expert group fell under the category "lab tests and chronic conditions." This was the least frequently mentioned category of effects by consumers, taking only $0.5 \%$ of consumers' mentions, and just over $7 \%$ of consumers mentioned an effect within this category at all. Within this category, Table 4.28 shows that most attention was given by experts and consumers to increased blood sugar and diabetes $(33 \%$ and $50 \%$ of mentions within the category, respectively). Effects on the liver were not mentioned in any expert text, but were reported by a small fraction of consumers $(0.8 \%)$. 
Table 4.28 also demonstrates an inconsistency in reporting among expert websites. RevolutionHealth only listed 1 of the 6 effects falling under this category, and did not attach a description or label to blood sugar high/diabetes. WebMD cited an increase in blood sugar as an infrequent effect in one paragraph and as a rare effect in another paragraph. On both occasions the effect was labeled severe or potentially severe. Other effects listed as infrequent or rare by WebMD were mentioned by relatively few consumers, but increased blood sugar/diabetes was mentioned by considerably more (4.8\% of consumers) and received much greater attention ( $50 \%$ of mentions within this category, and $1.7 \%$ of mentions within all drug effects).

For each of the 2 drugs, both experts and consumers gave the most attention to mental or mood effects. Consumers most frequently mentioned positive and negative changes in mental or mood symptoms, along with sleep and weight effects. Expert text more often attended to negative changes in mental or mood symptoms, such as suicide and agitation, along with other physical effects, like nausea/vomiting. Expert text tended to list possible mental or mood effects in summary form, while consumer text provided context and situational examples. There were clear and major differences in how effects were described by each group. For example, expert descriptions of "less serious" did not match consumer descriptions of "the worst" for certain weight and sexual effects. Similarly, expert frequency estimates of "common" and "infrequent" were not proportional to the rate of mentions in consumer text, especially for sleep effects of Seroquel and sexual effects of Lexapro. However, since the consumer rate of mention does not equal frequency of the effect, the latter finding might again only reflect consumers' perceived severity. 
Figure 4.9 Comparison of Frequency of Mentions of Seroquel Effects between Experts and Consumers, by Effect Category

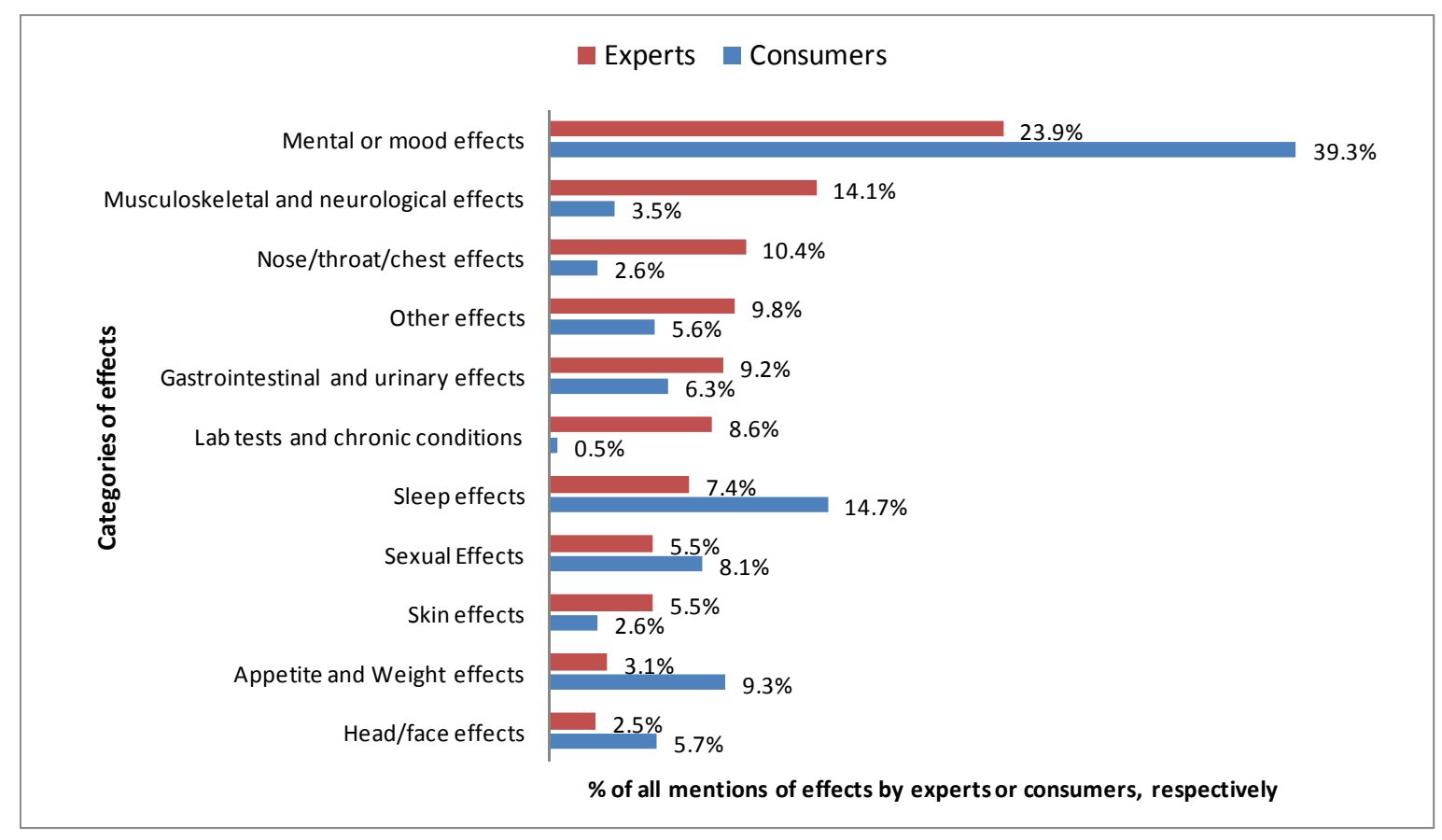


Figure 4.10 Comparison of Most Frequently Mentioned Seroquel Effects between Experts and Consumers

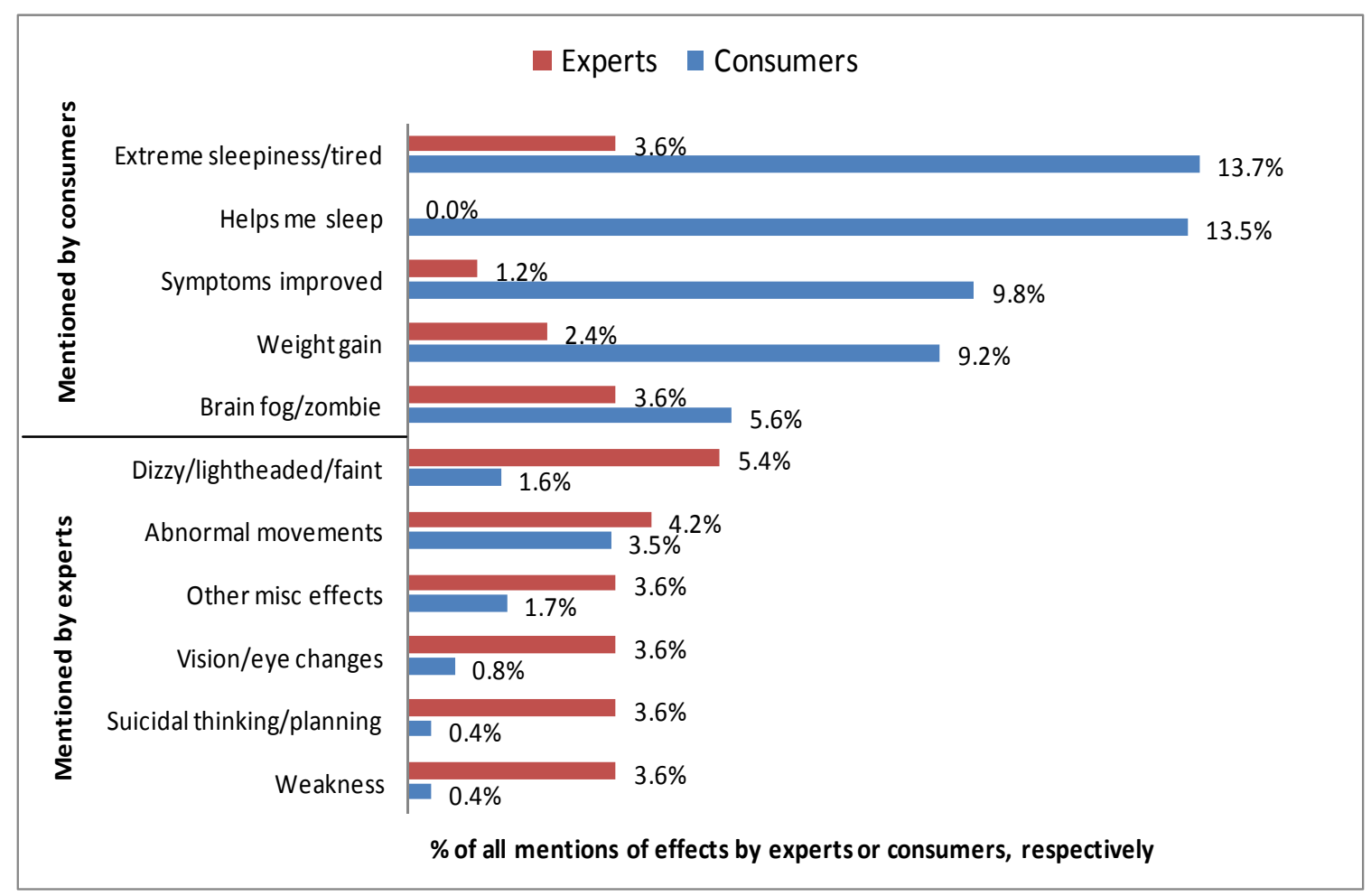


Table 4.25

Excerpts from Experts and Consumers on "Brain Fog/Zombie” Effect of Seroquel

\begin{tabular}{|c|c|}
\hline \multicolumn{2}{|r|}{ Effect: Brain fog/zombie } \\
\hline WebMD (Expert) & "Common side effects.... Difficulty speaking..............Severe" \\
\hline $\begin{array}{l}\text { RevHealth } \\
\text { (Expert) }\end{array}$ & $\begin{array}{l}\text { Quetiapine can cause side effects that may impair your thinking or } \\
\text { reactions" } \\
\text { "Other serious side effects include:... confusion, problems with } \\
\text { vision, speech, or balance" }\end{array}$ \\
\hline AskaPatient & $\begin{array}{l}\text { "With this drug I cannot function the next day, I have a foggy mind } \\
\text { and feel I just cannot move around" }(\# 514)\end{array}$ \\
\hline CrazyMeds & $\begin{array}{l}\text { "...slow, dumb, exhausted" (\#603) } \\
\text { "It just put me in total zombie land, and caused me to fall asleep with } \\
\text { my eyes open a few times" (\#657) } \\
\text { "I could intermittently find myself someplace and have no idea why I } \\
\text { was there or what I was doing... I have never experienced such a } \\
\text { profound blankness. I found myself in the walk-in closet, and had to } \\
\text { deduce why I might be in there... 'Ok, I am completely dressed, so } \\
\text { I'm not doing that..." (\#634) }\end{array}$ \\
\hline $\begin{array}{l}\text { RevHealth } \\
\text { (Consumers) }\end{array}$ & $\begin{array}{l}\text { "decreasing mental acuity, decreased gross motor function, excessive } \\
\text { fine motor tremor, your basic zombie" (\#747) } \\
\text { "my mind went all numb and I couldn't think straight" (\#781) }\end{array}$ \\
\hline $\begin{array}{l}\text { WebMD } \\
\text { (Consumers) }\end{array}$ & $\begin{array}{l}\text { "I wake up in the morning feeling sluggish and drugged. On some } \\
\text { days I have such a hard time shaking the fogginess off that I am } \\
\text { completely withdrawn" (\#905) } \\
\text { "It also makes it hard to communicate as well. If someone asks me } \\
\text { something after taking it I'm very incoherent" (\#941) }\end{array}$ \\
\hline
\end{tabular}


Table 4.26

Comparison of Experts and Consumers on "Sleep Effects" of Seroquel

\begin{tabular}{|c|c|c|c|c|c|}
\hline \multirow[b]{2}{*}{ Code } & \multicolumn{2}{|c|}{$\begin{array}{l}\% \text { of all mentions of } \\
\text { "sleep effects" }\end{array}$} & \multicolumn{2}{|c|}{ Expert description } & \multirow[b]{2}{*}{$\begin{array}{c}\% \text { of consumers } \\
\text { mentioning the } \\
\text { effect }\end{array}$} \\
\hline & Experts & Consumers & WebMD & $\begin{array}{c}\text { Rev } \\
\text { Health }\end{array}$ & \\
\hline $\begin{array}{l}\text { Extreme } \\
\text { sleepiness/ } \\
\text { tired }\end{array}$ & $66.7 \%$ & $45.1 \%$ & $\begin{array}{l}\text { "common" \& } \\
\text { "less severe" }\end{array}$ & $\begin{array}{c}\text { "less } \\
\text { serious" }\end{array}$ & $33.1 \%$ \\
\hline $\begin{array}{l}\text { Helps me } \\
\text { sleep }\end{array}$ & $0 \%$ & $44.7 \%$ & -- & -- & $35.6 \%$ \\
\hline $\begin{array}{l}\text { Trouble } \\
\text { sleeping/ } \\
\text { insomnia }\end{array}$ & $33.3 \%$ & $2.6 \%$ & $\begin{array}{l}\text { "common" \& } \\
\text { "less severe" }\end{array}$ & $\begin{array}{c}\text { No } \\
\text { description }\end{array}$ & $2.3 \%$ \\
\hline $\begin{array}{l}\text { Difficult to } \\
\text { wake up }\end{array}$ & $0 \%$ & $7.7 \%$ & -- & -- & $6.3 \%$ \\
\hline
\end{tabular}


Table 4.27

Excerpts from Experts and Consumers on Selected "Sleep Effects" of Seroquel

\begin{tabular}{|c|c|}
\hline \multicolumn{2}{|r|}{ Effects: Helps me sleep; Extreme sleepiness/tired } \\
\hline WebMD (Expert) & $\begin{array}{l}\text { "Common side effects: drowsiness...............Less Severe" } \\
\text { "tiredness" }\end{array}$ \\
\hline $\begin{array}{l}\text { RevHealth } \\
\text { (Expert) }\end{array}$ & $\begin{array}{l}\text { "The following warnings are available for this medication:.... May } \\
\text { cause drowsiness" }\end{array}$ \\
\hline AskaPatient & $\begin{array}{l}\text { "So while it does provide me sleep...it's the kind of sleep that } \\
\text { wouldn't allow me to be woken, even if my house is on fire. I am not } \\
\text { able to be woken from this coma like sleep for hours. That scares } \\
\text { me." (case \#515) } \\
\text { "extreme sleeping" (\#570) }\end{array}$ \\
\hline CrazyMeds & $\begin{array}{l}\text { "I like what this drug does to me (sleepy bye bye land)" (\#613) } \\
\text { "You'll sleep until next Tuesday. Of course, that could be a good } \\
\text { thing, depending on how your life is at this moment" (\#1084) }\end{array}$ \\
\hline RevolutionHealth & $\begin{array}{l}\text { "Seroquel puts me to sleep. It's that simple. I take it and within an } \\
\text { hour I'm out - unwakable - for the next } 12 \text { or more hours" (\#739) } \\
\text { "helped very, very effectively with sleep: } 30 \text { minutes max after } \\
\text { taking 125-150mg at night, I am out for good" (\#808) } \\
\text { "the worst side effect is the sleepiness - I sleep 10-12 hours a day } \\
\text { and still have periods when I have to nap (or could fall asleep } \\
\text { standing up)" (\#773) }\end{array}$ \\
\hline WebMD & $\begin{array}{l}\text { "It helped me sleep very well, but I was very groggy in the morning" } \\
(\# 871)\end{array}$ \\
\hline
\end{tabular}


Table 4.28

Comparison of Experts and Consumers on "Lab Tests and Chronic Conditions" Mentioned for Seroquel

\begin{tabular}{|c|c|c|c|c|c|}
\hline & \multicolumn{2}{|c|}{$\begin{array}{l}\% \text { of all mentions of } \\
\text { "lab tests and } \\
\text { chronic conditions" }\end{array}$} & \multicolumn{2}{|c|}{ Expert description } & \multirow{2}{*}{$\begin{array}{l}\% \text { of } \\
\text { consumers } \\
\text { mentioning } \\
\text { the effect }\end{array}$} \\
\hline & Expert & Consumers & WebMD & $\begin{array}{c}\text { Rev } \\
\text { Health }\end{array}$ & \\
\hline $\begin{array}{l}\text { Blood sugar } \\
\text { high/diabetes }\end{array}$ & $33.3 \%$ & $51.0 \%$ & $\begin{array}{l}\text { "infrequent"//rare" } \\
\text { \& "severe" (Blood } \\
\text { sugar); } \\
\text { "rare" \& "severe" } \\
\text { (Diabetes) }\end{array}$ & $\begin{array}{c}\text { Not } \\
\text { described }\end{array}$ & $4.8 \%$ \\
\hline $\begin{array}{l}\text { Other labs or } \\
\text { chronic conditions }\end{array}$ & $26.7 \%$ & $13.7 \%$ & $\begin{array}{l}\text { "infrequent"/"rare" } \\
\& \text { "less severe"/ } \\
\text { "severe" }\end{array}$ & -- & $1.5 \%$ \\
\hline $\begin{array}{l}\text { Blood related } \\
\text { changes }\end{array}$ & $13.3 \%$ & $2.0 \%$ & "rare" \& "severe" & -- & $0.2 \%$ \\
\hline Cholesterol high & $13.3 \%$ & $11.8 \%$ & $\begin{array}{l}\text { "infrequent" \& } \\
\text { "less severe" }\end{array}$ & -- & $0.6 \%$ \\
\hline $\begin{array}{l}\text { Blood pressure } \\
\text { changes }\end{array}$ & $13.3 \%$ & $11.8 \%$ & $\begin{array}{l}\text { "common"/ } \\
\text { "infrequent" \& } \\
\text { "less } \\
\text { severe"/“severe" }\end{array}$ & -- & $1.3 \%$ \\
\hline $\begin{array}{l}\text { Liver } \\
\text { levels/hepatoxicity/ } \\
\text { hepatitis }\end{array}$ & $0 \%$ & $9.8 \%$ & -- & -- & $0.8 \%$ \\
\hline
\end{tabular}




\section{Drug Effects by Variables}

\section{Lexapro and gender.}

Male and female consumers reported similar effects of Lexapro across all drug effect categories, except for sexual effects (Table 4.29) where more males (41.6\%) than females (17.1\%) mentioned an effect. Table 4.30 compares consumers who did and did not report gender across drug effect categories, and shows a significant difference at the $p<0.002$ level (corrected for multiple comparisons) for appetite and weight effects, and mental or mood effects. Each category was reported more frequently by consumers who did report gender. Table 4.31 further compares each gender to those who did not report gender for 3 categories of effects. Both appetite and weight effects, and mental or mood effects were significantly different only for females ( $\mathrm{p}=0.001$ and 0.000 , respectively). Sexual effects were tested because they approached significance in the previous comparison, and were found here to only significantly differ for males $(\mathrm{p}=0.000)$.

\section{Lexapro and age.}

There were no significant differences in the proportion of consumers in 3 age brackets (7-24, 25-44, and 45+) who mentioned an effect of Lexapro according to effect category (Table 4.32). Considerably fewer consumers in the $45+$ age bracket (56.9\%) reported mental or mood effects compared to 7-24 year old consumers (75.6\%) and 25-44 year old consumers (73.3\%). Each age group reported appetite and weight effects more frequently than those who did not report their age, though the rate was highest for the youngest age group (33.3\%). Other differences included a slightly higher reporting of sleep effects in the youngest age group (51.1\%) and middle age group (43.5\%), compared to the oldest age group (31.9\%) and those who did not report their age (29.7\%). The middle age group 
had the highest proportion of consumers reporting musculoskeletal and neurological effects (18.3\%), compared to the youngest age group (8.9\%) and those who did not report their age (9.1\%). Table 4.33 compares consumers who did and did not report age across drug effect categories. Two categories reached statistical significance at the $\mathrm{p}<0.003$ level (corrected for multiple comparisons), and in each case more consumers who did provide their age reported effects in these categories.

\section{Lexapro and length of time taken.}

Table 4.34 compares reported effects of Lexapro in effect categories by the length of time the consumer had been taking the drug. To minimize empty and small cells, time categories were collapsed into 2 groups: consumers taking the drug for less than 6 months, and longer than 6 months. Three of the 10 effect categories were significantly different between groups, and each was reported more frequently by consumers taking Lexapro for less than 6 months. These categories included "side effects" that would be expected to resolve over time. Also, it would be unlikely for individuals experiencing many of these effects to remain on the drug if the effects persisted for longer than 6 months. Most of the categories that did not show a significant difference between groups, particularly sexual effects, appetite and weight effects, and mental or mood effects, would generally be sustained over time and not expected to disappear as part of the initial "side effects" of Lexapro.

Table 4.35 compares drug effect categories by consumers who did and did not report how long they had been taking Lexapro. Three of the categories were significantly different between groups at the $\mathrm{p}<0.003$ level. Similar to the gender and age variables, 
consumers who did provide the length of time taking Lexapro reported these effects at a higher rate than those who did not provide that information.

Table 4.29

Gender of Consumers who Mentioned an Effect of Lexapro According to Drug Effect Category $^{a}$

\begin{tabular}{|l|c|c|c|c|}
\hline & $\begin{array}{c}\text { Female } \\
(\mathrm{N}=215)\end{array}$ & $\begin{array}{c}\text { Male } \\
(\mathrm{N}=77)\end{array}$ & $\begin{array}{c}\text { Chi- } \\
\text { square }\end{array}$ & $\begin{array}{c}\mathrm{p}- \\
\text { value }\end{array}$ \\
\hline Sexual Effects & $37(17.1 \%)$ & $32(41.6 \%)$ & 18.816 & 0.000 \\
\hline Appetite and Weight Effects & 63 & 16 & 2.028 & 0.154 \\
\hline Other Effects & 34 & 8 & 1.324 & 0.250 \\
\hline $\begin{array}{l}\text { Musculoskeletal and Neurological } \\
\text { Effects }\end{array}$ & 28 & 13 & 0.725 & 0.395 \\
\hline Mental or Mood Effects & 154 & 51 & 0.692 & 0.405 \\
\hline Head or Face Effects & 30 & 13 & 0.406 & 0.524 \\
\hline Nose Throat Chest Effects & 18 & 8 & 0.297 & 0.586 \\
\hline Gastrointestinal and Urinary Effects & 35 & 12 & 0.016 & 0.899 \\
\hline Sleep Effects & 83 & 30 & 0.007 & 0.934 \\
\hline
\end{tabular}

${ }^{\mathrm{a}} \mathrm{p}$-value set at 0.002 for $\mathrm{k}=25$ comparisons of gender in Tables $4.29-4.31$ 
Table 4.30

Reporting of Gender and Drug Effect Category for Lexapro ${ }^{a}$

\begin{tabular}{|l|c|c|c|c|}
\hline & $\begin{array}{c}\text { Gender given } \\
(\mathrm{N}=292)\end{array}$ & $\begin{array}{c}\text { Gender not given } \\
(\mathrm{N}=188)\end{array}$ & $\begin{array}{c}\text { Chi- } \\
\text { square }\end{array}$ & $\begin{array}{c}\text { p- } \\
\text { value }\end{array}$ \\
\hline Mental or Mood Effects & $205(70.0 \%)$ & $91(48.7 \%)$ & 21.914 & 0.000 \\
\hline Appetite and Weight Effects & $79(27.0 \%)$ & $28(15.0 \%)$ & 9.472 & 0.002 \\
\hline Sexual Effects & $69(23.5 \%)$ & $27(14.4 \%)$ & 5.922 & 0.015 \\
\hline Sleep Effects & 113 & 59 & 2.444 & 0.118 \\
\hline $\begin{array}{l}\text { Musculoskeletal and } \\
\text { Neurological Effects }\end{array}$ & 41 & 18 & 2.02 & 0.155 \\
\hline Other Effects & 25 & 34 & 1.268 & 0.260 \\
\hline Skin Effects & 26 & 11 & 1.156 & 0.282 \\
\hline Nose Throat Chest Effects & 43 & 24 & 0.945 & 0.331 \\
\hline Head or Face Effects & 42 & 12 & 0.095 & 0.758 \\
\hline $\begin{array}{l}\text { Gastrointestinal and Urinary } \\
\text { Effects }\end{array}$ & 26 & & 0.570 \\
\hline
\end{tabular}

${ }^{\mathrm{a}} \mathrm{p}$-value set at 0.002 for $\mathrm{k}=25$ comparisons of gender in Tables $4.29-4.31$ 
Table 4.31

Male and Female Consumers Compared to Those Who Did Not Report Gender on Drug Effects for Selected Categories of Lexapro ${ }^{a}$

\begin{tabular}{|l|c|c|c|c|}
\hline & $\begin{array}{c}\text { Female } \\
(\mathrm{N}=215)\end{array}$ & $\begin{array}{c}\text { Gender not given } \\
(\mathrm{N}=188)\end{array}$ & $\begin{array}{c}\text { Chi- } \\
\text { square }\end{array}$ & $\begin{array}{c}\mathrm{p} \text { - } \\
\text { value }\end{array}$ \\
\hline $\begin{array}{l}\text { Appetite and Weight } \\
\text { Effects }\end{array}$ & $63(29.2 \%)$ & $28(15.0 \%)$ & 11.55 & 0.001 \\
\hline Mental or Mood Effects & $154(71.3 \%)$ & $91(48.7 \%)$ & 21.541 & 0.000 \\
\hline Sexual Effects & 37 & 27 & 0.543 & 0.461 \\
\hline $\begin{array}{l}\text { Male } \\
\text { Effects }\end{array}$ & $\begin{array}{c}\text { Gender not given } \\
(\mathrm{N}=18)\end{array}$ & 28 & 1.324 & 0.25 \\
\hline Mental or Mood Effects & $51(66.2 \%)$ & $91(48.7 \%)$ & 6.774 & 0.009 \\
\hline Sexual Effects & $32(41.6 \%)$ & $27(14.4 \%)$ & 23.116 & 0.000 \\
\hline
\end{tabular}

${ }^{\mathrm{a}} \mathrm{p}$-value set at 0.002 for $\mathrm{k}=25$ comparisons of gender in Tables $4.29-4.31$ 
Table 4.32

Age of Consumers Who Mentioned an Effect of Lexapro According to Drug Effect Category $^{a}$

\begin{tabular}{|l|c|c|c|c|c|}
\hline & $\begin{array}{c}7-24 \text { yrs } \\
(\mathrm{n}=45)\end{array}$ & $\begin{array}{c}25-44 \mathrm{yrs} \\
(\mathrm{n}=131)\end{array}$ & $\begin{array}{c}45+\mathrm{yrs} \\
(\mathrm{n}=72)\end{array}$ & $\begin{array}{c}\text { Chi- } \\
\text { square }\end{array}$ & $\begin{array}{c}\mathrm{p}- \\
\text { value }\end{array}$ \\
\hline Mental or Mood Effects & $34(75.6 \%)$ & $96(73.3 \%)$ & $41(56.9 \%)$ & 6.913 & 0.032 \\
\hline Sleep Effects & $23(51.1 \%)$ & $57(43.5 \%)$ & $23(31.9 \%)$ & 4.637 & 0.098 \\
\hline Head or Face Effects & 9 & 22 & 7 & 2.718 & 0.257 \\
\hline $\begin{array}{l}\text { Musculoskeletal and } \\
\text { Neurological Effects }\end{array}$ & $4(8.9 \%)$ & $24(18.3 \%)$ & $10(13.9 \%)$ & -- & -- \\
\hline Other Effects & 4 & 21 & 14 & -- & -- \\
\hline Sexual Effects & 13 & 32 & 13 & 1.982 & 0.371 \\
\hline Skin Effects & 6 & 11 & 5 & 1.475 & 0.478 \\
\hline $\begin{array}{l}\text { Appetite and Weight } \\
\text { Effects }\end{array}$ & $15(33.3 \%)$ & $37(28.2 \%)$ & $17(23.6 \%)$ & 1.328 & 0.515 \\
\hline $\begin{array}{l}\text { Gastrointestinal and } \\
\text { Urinary Effects }\end{array}$ & 5 & 11 & 9 & 0.927 & 0.629 \\
\hline Nose Throat Chest Effects & 5 & 10 & 0.992 & 0.609 \\
\hline
\end{tabular}

${ }^{a} \mathrm{p}$-values set at 0.003 for $\mathrm{k}=18$ comparisons involving age in Tables 4.32 and 4.33 
Table 4.33

Reporting of Age by Drug Effect Category for Lexapro ${ }^{a}$

\begin{tabular}{|c|c|c|c|c|}
\hline & $\begin{array}{l}\text { Age given } \\
(\mathrm{n}=248)\end{array}$ & $\begin{array}{l}\text { Age not given } \\
\qquad(\mathrm{n}=232)\end{array}$ & $\begin{array}{l}\text { Chi- } \\
\text { square }\end{array}$ & $\begin{array}{c}\mathrm{p}- \\
\text { value }\end{array}$ \\
\hline Mental or Mood Effects & $171(69.0 \%)$ & $125(53.9 \%)$ & 11.519 & 0.001 \\
\hline Appetite and Weight Effects & $69(27.8 \%)$ & $38(16.4 \%)$ & 9.061 & 0.003 \\
\hline Sleep Effects & $103(41.5 \%)$ & $69(29.7 \%)$ & 7.248 & 0.007 \\
\hline $\begin{array}{l}\text { Musculoskeletal and } \\
\text { Neurological Effects }\end{array}$ & $38(15.3 \%)$ & $21(9.1 \%)$ & 4.372 & 0.037 \\
\hline Sexual Effects & 58 & 38 & 3.679 & 0.055 \\
\hline Nose Throat Chest Effects & 25 & 13 & 3.296 & 0.069 \\
\hline Skin Effects & 22 & 14 & 1.39 & 0.238 \\
\hline Head or Face Effects & 38 & 29 & 0.795 & 0.373 \\
\hline $\begin{array}{l}\text { Gastrointestinal and Urinary } \\
\text { Effects }\end{array}$ & 40 & 39 & 0.04 & 0.841 \\
\hline Other Effects & 39 & 37 & 0.004 & 0.947 \\
\hline
\end{tabular}

${ }^{a} \mathrm{p}$-values set at 0.003 for $\mathrm{k}=18$ comparisons involving age in Tables 4.32 and 4.33 
Table 4.34

Consumers Taking Lexapro According to Length of Time Taken and Drug Effect Category $^{a}$

\begin{tabular}{|l|c|c|c|c|}
\hline & $\begin{array}{c}\text { Taken }<6 \text { months } \\
(\mathrm{n}=228)\end{array}$ & $\begin{array}{c}\text { Taken }>6 \text { months } \\
(\mathrm{n}=140)\end{array}$ & $\begin{array}{c}\text { Chi- } \\
\text { square }\end{array}$ & $\begin{array}{c}\mathrm{p} \text { - } \\
\text { value }\end{array}$ \\
\hline $\begin{array}{l}\text { Musculoskeletal and } \\
\text { Neurological Effects }\end{array}$ & $45(19.7 \%)$ & $9(6.4 \%)$ & 12.27 & 0.000 \\
\hline $\begin{array}{l}\text { Gastrointestinal and Urinary } \\
\text { Effects }\end{array}$ & $59(25.9 \%)$ & $7(5.0 \%)$ & 25.686 & 0.000 \\
\hline Head or Face Effects & $50(21.9 \%)$ & $9(6.4 \%)$ & 15.482 & 0.000 \\
\hline Sleep Effects & $107(46.9 \%)$ & $45(32.1 \%)$ & 7.823 & 0.005 \\
\hline Nose Throat Chest Effects & $28(12.3 \%)$ & $7(5.0 \%)$ & 5.343 & 0.021 \\
\hline Skin Effects & $26(11.4 \%)$ & $7(5.0 \%)$ & 4.357 & 0.037 \\
\hline Sexual Effects & 46 & 35 & 1.176 & 0.278 \\
\hline Appetite and Weight Effects & 63 & 36 & 0.162 & 0.687 \\
\hline Mental or Mood Effects & 159 & 95 & 0.143 & 0.705 \\
\hline Other Effects & 37 & 24 & 0.052 & 0.819 \\
\hline
\end{tabular}

${ }^{a} \mathrm{p}$-values set at 0.003 for $\mathrm{k}=18$ comparisons involving time taken in Tables 4.34 and 4.35 
Table 4.35

Reporting Length of Time Taking Lexapro According to Drug Effect Category ${ }^{a}$

\begin{tabular}{|l|c|c|c|c|}
\hline & $\begin{array}{c}\text { Time taken } \\
\text { given }(\mathrm{n}=368)\end{array}$ & $\begin{array}{c}\text { Time taken not } \\
\text { given }(\mathrm{n}=112)\end{array}$ & $\begin{array}{c}\text { Chi- } \\
\text { square }\end{array}$ & $\begin{array}{c}\mathrm{p}- \\
\text { value }\end{array}$ \\
\hline Appetite and Weight Effects & $99(26.9 \%)$ & $8(7.1 \%)$ & 19.353 & 0.000 \\
\hline Sleep Effects & $152(41.3 \%)$ & $20(17.9 \%)$ & 20.531 & 0.000 \\
\hline Mental or Mood Effects & $254(69.0 \%)$ & $42(37.5 \%)$ & 36.093 & 0.000 \\
\hline $\begin{array}{l}\text { Musculoskeletal and } \\
\text { Neurological Effects }\end{array}$ & $54(14.7 \%)$ & $5(4.5 \%)$ & 8.302 & 0.004 \\
\hline Head or Face Effects & $59(16.0 \%)$ & $8(7.1 \%)$ & 5.65 & 0.017 \\
\hline Nose Throat Chest Effects & 35 & 3 & -- & -- \\
\hline Skin Effects & 33 & 3 & -- & -- \\
\hline Sexual Effects & 61 & 15 & 0.653 & 0.419 \\
\hline Other Effects & 61 & 15 & & \\
\hline
\end{tabular}

${ }^{a} \mathrm{p}$-values set at 0.003 for $\mathrm{k}=18$ comparisons involving time taken in Tables 4.34 and 4.35

\section{Seroquel and gender.}

Male and female consumers did not differ in reported effects (grouped by category) for Seroquel (Table 4.36). Table 4.37 further shows no differences at the $\mathrm{p}<0.003$ level between consumers who did give their gender compared to those who did not give that information.

\section{Seroquel and age.}

Reported effects of Seroquel grouped by category did not differ significantly $(p<0.003)$ between the age brackets of 7-24, 25-44, and 45+ years old (Table 4.38) or between 
consumers who did report their age and those who did not report that information (Table 4.39). Similar to trends in Lexapro data, the youngest age category mentioned appetite and weight effects (40.9\%) and sleep effects (72.7\%) more often than the other age groups and those who did not report age.

Seroquel and length of time taken.

Table 4.40 compares effect categories for Seroquel by the length of time consumers had taken the drug (less than and more than 6 months). Significantly more consumers taking Seroquel for longer than 6 months mentioned appetite and weight effects $(44.7 \%$ versus $27.5 \%)$ and lab tests and chronic conditions (13.7\% versus $3.0 \%)$ at the $\mathrm{p}<0.003$ level. Two effect categories were significantly different between consumers who did provide the length of time taking Seroquel and those who did not give that information (Table 4.41). Consumers taking Seroquel for less than or more than 6 months all reported sleep effects (62.9\% and 69.6\%, respectively) at a higher rate than consumers who did not provide the time taken $(48.7 \%)$.

In sum, a high number of consumers not reporting the above variables prevented a detailed examination of differences in drug effects by gender, age, and time taken. Instead, comparisons were made according to broader drug effect categories. The primary finding was that the more information consumers provided, the more likely the consumer was to report a drug effect. In general, there were fewer differences for Seroquel than for Lexapro in reported effects by each of the 3 variables. Men were more likely than women to mention sexual effects of Lexapro. There were no differences between the genders for effects of Seroquel. The youngest age group tended to more frequently mention sleep and weight effects for both drugs. Finally, more consumers reported what appeared to be 
initial "side effects" of Lexapro, whereas more reported sustained effects lasting longer than 6 months (i.e., weight and blood sugar) of Seroquel.

Table 4.36

Gender of Consumers Who Mentioned an Effect of Seroquel According to Drug Effect Category $^{a}$

\begin{tabular}{|l|c|c|c|c|}
\hline & $\begin{array}{c}\text { Female } \\
(\mathrm{N}=207)\end{array}$ & $\begin{array}{c}\text { Male } \\
(\mathrm{N}=91)\end{array}$ & $\begin{array}{c}\text { Chi- } \\
\text { square }\end{array}$ & $\begin{array}{c}\mathrm{p}- \\
\text { value }\end{array}$ \\
\hline $\begin{array}{l}\text { Musculoskeletal and Neurological } \\
\text { Effects }\end{array}$ & 51 & 14 & 3.173 & 0.075 \\
\hline Other Effects & 37 & 10 & 2.256 & 0.133 \\
\hline Head or Face Effects & 11 & 9 & 2.114 & 0.146 \\
\hline Skin Effects & 12 & 2 & -- & -- \\
\hline Sexual Effects & $2(1.0 \%)$ & $2(2.2 \%)$ & 0.724 & 0.395 \\
\hline Gastrointestinal and Urinary Effects & 14 & 4 & -- & -- \\
\hline Sleep Effects & 132 & 54 & 0.528 & 0.467 \\
\hline Mental or Mood Effects & 111 & 52 & 0.316 & 0.574 \\
\hline Nose Throat Chest Effects & 26 & 10 & 0.147 & 0.701 \\
\hline Lab Tests and Chronic Conditions & 18 & 9 & 0.109 & 0.741 \\
\hline Appetite and Weight Effects & $70(33.8 \%)$ & $31(34.1 \%)$ & 0.002 & 0.967 \\
\hline
\end{tabular}

${ }^{a} \mathrm{p}$-values set at 0.002 for $\mathrm{k}=18$ comparisons involving gender in Tables 4.36 and 4.37 
Table 4.37

Reporting Gender by Drug Effect Category for Seroquel ${ }^{a}$

\begin{tabular}{|c|c|c|c|c|}
\hline & $\begin{array}{l}\text { Gender given } \\
\qquad(\mathrm{N}=298)\end{array}$ & $\begin{array}{l}\text { Gender not given } \\
\qquad(\mathrm{N}=182)\end{array}$ & $\begin{array}{l}\text { Chi- } \\
\text { square }\end{array}$ & $\begin{array}{c}\mathrm{p}- \\
\text { value }\end{array}$ \\
\hline $\begin{array}{l}\text { Musculoskeletal and } \\
\text { Neurological Effects }\end{array}$ & $65(21.8 \%)$ & $22(12.1 \%)$ & 7.2 & 0.007 \\
\hline $\begin{array}{l}\text { Lab Tests and Chronic } \\
\text { Conditions }\end{array}$ & $27(9.1 \%)$ & $7(3.8 \%)$ & 4.668 & 0.031 \\
\hline Skin Effects & 14 & 2 & -- & -- \\
\hline Appetite and Weight Effects & 101 & 47 & 3.449 & 0.063 \\
\hline $\begin{array}{l}\text { Gastrointestinal and Urinary } \\
\text { Effects }\end{array}$ & 18 & 6 & 1.791 & 0.181 \\
\hline Nose Throat Chest Effects & 36 & 15 & 1.753 & 0.185 \\
\hline Head or Face Effects & 20 & 7 & 1.747 & 0.186 \\
\hline Sleep Effects & 186 & 105 & 1.056 & 0.304 \\
\hline Mental or Mood Effects & 163 & 91 & 1.001 & 0.317 \\
\hline Sexual Effects & 4 & 2 & -- & -- \\
\hline Other Effects & 47 & 30 & 0.042 & 0.837 \\
\hline
\end{tabular}

${ }^{\mathrm{a}} \mathrm{p}$-values set at 0.003 for $\mathrm{k}=18$ comparisons involving gender in Tables 4.36 and 4.37 
Table 4.38

Age of Consumers Who Mentioned an Effect of Seroquel According to Drug Effect Category $^{a}$

\begin{tabular}{|l|c|c|c|c|c|}
\hline & $\begin{array}{c}7-24 \mathrm{yrs} \\
(\mathrm{n}=44)\end{array}$ & $\begin{array}{c}25-44 \mathrm{yrs} \\
(\mathrm{n}=119)\end{array}$ & $\begin{array}{c}45-75+\mathrm{yrs} \\
(\mathrm{n}=82)\end{array}$ & $\begin{array}{c}\text { Chi- } \\
\text { square }\end{array}$ & $\begin{array}{c}\mathrm{p}- \\
\text { value }\end{array}$ \\
\hline $\begin{array}{l}\text { Appetite and Weight } \\
\text { Effects }\end{array}$ & $18(40.9 \%)$ & $41(34.5 \%)$ & $29(35.4 \%)$ & 0.598 & 0.742 \\
\hline $\begin{array}{l}\text { Gastrointestinal and } \\
\text { Urinary Effects }\end{array}$ & 6 & 10 & 2 & -- & -- \\
\hline Head or Face Effects & 5 & 4 & 6 & 3.486 & 0.175 \\
\hline $\begin{array}{l}\text { Lab Tests and Chronic } \\
\text { Conditions }\end{array}$ & 25 & 68 & 42 & 0.752 & 0.686 \\
\hline Mental or Mood Effects & 9 & 29 & 13 & 2.14 & 0.343 \\
\hline $\begin{array}{l}\text { Musculoskeletal and } \\
\text { Neurological Effects }\end{array}$ & $32(72.7 \%)$ & $74(62.2 \%)$ & $52(63.4 \%)$ & 1.621 & 0.445 \\
\hline Nose Throat Chest Effects & $7(15.9 \%)$ & $14(11.8 \%)$ & $12(14.6 \%)$ & 0.617 & 0.735 \\
\hline Other Effects & 5 & 21 & 10 & 1.626 & 0.444 \\
\hline Skin Effects & 2 & 6 & 4 & -- & - \\
\hline Sleep Effects & 6 & & & & - \\
\hline
\end{tabular}

${ }^{a} \mathrm{p}$-values set at 0.003 for $\mathrm{k}=16$ comparisons involving age in Tables 4.38 and 4.39 
Table 4.39

Reporting Age by Drug Effect Category for Seroquel ${ }^{a}$

\begin{tabular}{|l|c|c|c|c|}
\hline & $\begin{array}{c}\text { Age given } \\
(\mathrm{n}=245)\end{array}$ & $\begin{array}{c}\text { Age not given } \\
(\mathrm{n}=235)\end{array}$ & $\begin{array}{c}\text { Chi- } \\
\text { square }\end{array}$ & $\begin{array}{c}\mathrm{p}- \\
\text { value }\end{array}$ \\
\hline Appetite and Weight Effects & $88(35.9 \%)$ & $60(25.5 \%)$ & 6.068 & 0.014 \\
\hline Nose Throat Chest Effects & $33(13.5 \%)$ & $18(7.7 \%)$ & 4.264 & 0.039 \\
\hline Skin Effects & 12 & 4 & -- & -- \\
\hline Sleep Effects & 158 & 133 & 3.131 & 0.077 \\
\hline Head or Face Effects & 18 & 9 & 2.795 & 0.095 \\
\hline $\begin{array}{l}\text { Musculoskeletal and } \\
\text { Neurological Effects }\end{array}$ & 131 & 36 & 2.443 & 0.118 \\
\hline Mental or Mood Effects & 36 & 119 & 0.959 & 0.327 \\
\hline $\begin{array}{l}\text { Other Effects } \\
\text { Gastrointestinal and Urinary } \\
\text { Effects }\end{array}$ & 19 & 10 & 0.675 & 0.411 \\
\hline $\begin{array}{l}\text { Lab Tests and Chronic } \\
\text { Conditions }\end{array}$ & 15 & 0.343 & 0.558 \\
\hline
\end{tabular}

${ }^{a} \mathrm{p}$-values set at 0.003 for $\mathrm{k}=16$ comparisons involving age in Tables 4.38 and 4.39 
Table 4.40

Consumers Taking Seroquel by Length of Time Taken and Drug Effect Category ${ }^{a}$

\begin{tabular}{|l|c|c|c|c|}
\hline & $\begin{array}{c}\text { Taken }<6 \text { months } \\
(\mathrm{n}=167)\end{array}$ & $\begin{array}{c}\text { Taken }>6 \text { months } \\
(\mathrm{n}=161)\end{array}$ & $\begin{array}{c}\text { Chi- } \\
\text { square }\end{array}$ & $\begin{array}{c}\mathrm{p}- \\
\text { value }\end{array}$ \\
\hline $\begin{array}{l}\text { Lab Tests and Chronic } \\
\text { Conditions }\end{array}$ & $5(3.0 \%)$ & $22(13.7 \%)$ & 12.356 & 0.000 \\
\hline Appetite and Weight Effects & $46(27.5 \%)$ & $72(44.7 \%)$ & 10.499 & 0.001 \\
\hline $\begin{array}{l}\text { Musculoskeletal and } \\
\text { Neurological Effects }\end{array}$ & $40(24.0 \%)$ & $28(17.4 \%)$ & 2.147 & 0.143 \\
\hline $\begin{array}{l}\text { Gastrointestinal and Urinary } \\
\text { Effects }\end{array}$ & 13 & 7 & 1.691 & 0.193 \\
\hline Sleep Effects & $105(62.9 \%)$ & $112(69.6 \%)$ & 1.639 & 0.2 \\
\hline Nose Throat Chest Effects & $25(15.0 \%)$ & $19(11.8 \%)$ & 0.709 & 0.4 \\
\hline Other Effects & 26 & 30 & 0.544 & 0.461 \\
\hline Mental or Mood Effects & 96 & 87 & 0.395 & 0.53 \\
\hline Skin Effects & 7 & 9 & 0.345 & 0.557 \\
\hline Head or Face Effects & 2 & 12 & 0.009 & 0.926 \\
\hline Sexual Effects & 72 & -- & - \\
\hline
\end{tabular}

${ }^{\mathrm{a}} \mathrm{p}$-values set at 0.003 for $\mathrm{k}=17$ comparisons involving time taken in Tables 4.40 and 4.41 
Table 4.41

Reporting Length of Time Taking Seroquel by Drug Effect Category ${ }^{a}$

\begin{tabular}{|c|c|c|c|c|}
\hline & $\begin{array}{c}\text { Time taken } \\
\text { given }(\mathrm{n}=328)\end{array}$ & $\begin{array}{l}\text { Time taken not } \\
\text { given }(n=152)\end{array}$ & $\begin{array}{l}\text { Chi- } \\
\text { square }\end{array}$ & $\begin{array}{c}\mathrm{p}- \\
\text { value }\end{array}$ \\
\hline Appetite and Weight Effects & $118(36.0 \%)$ & $30(19.7 \%)$ & 12.843 & 0.000 \\
\hline Sleep Effects & $217(66.2 \%)$ & $74(48.7 \%)$ & 13.286 & 0.000 \\
\hline Nose Throat Chest Effects & $44(13.4 \%)$ & $7(4.6 \%)$ & 8.488 & 0.004 \\
\hline $\begin{array}{l}\text { Musculoskeletal and } \\
\text { Neurological Effects }\end{array}$ & $68(20.7 \%)$ & $19(12.5 \%)$ & 4.743 & 0.029 \\
\hline Skin Effects & 16 & 0 & -- & -- \\
\hline Head or Face Effects & 24 & 3 & -- & -- \\
\hline Mental or Mood Effects & 183 & 71 & 3.439 & 0.064 \\
\hline $\begin{array}{l}\text { Gastrointestinal and Urinary } \\
\text { Effects }\end{array}$ & 20 & 4 & -- & -- \\
\hline $\begin{array}{l}\text { Lab Tests and Chronic } \\
\text { Conditions }\end{array}$ & 27 & 7 & 2.075 & 0.15 \\
\hline Other Effects & 56 & 21 & 0.818 & 0.366 \\
\hline Sexual Effects & 4 & 2 & -- & -- \\
\hline
\end{tabular}

${ }^{\mathrm{a}} \mathrm{p}$-values set at 0.003 for $\mathrm{k}=17$ comparisons involving time taken in Tables 4.40 and 4.41

\section{Themes Mentioned by Consumers}

Overview of theme categories.

Themes around drugs and drug use were grouped in the umbrella category "My Drug," which was divided into 7 main categories under which codes fell (see codebook in Appendix D). While coding for drug effects was restricted to Lexapro and Seroquel only, 
use of codes within "My Drug" were open to any general or specific comments about drug use. The first category was "Assessing the Overall Experience," reflecting consumers' weighing of benefits and costs of the drug. Two codes in this category, "Lexapro/Seroquel is a miracle/saved my life" and "I hate Lexapro/Seroquel," were the only codes under "My Drug" that solely included comments specific to the drugs under study (Table 4.42). The second category was "Dealing with Side Effects/Finding Optimum Effectiveness," reflecting consumers' attempts and strategies for counteracting drugs' side effects and/or for enhancing drugs' effectiveness. The next category, "Do I Need Meds?" included consumers' comments about wanting to remain on or quit drug treatment for their problems, desires or attempts at alternative non-drug treatments, and reflections on how drugs have affected them. The fourth category, "Evidence," involved consumers' attempts to decipher the exact nature and cause of a particular effect they experienced, and also included comments about the lack of research on a use, effect, or other aspect of a drug. The "Other Drug Issues" category included various other codes involving drug dependence and withdrawal, dosing issues, miscellaneous descriptions of symptoms related to a diagnosis, comparisons of brand versus generic drugs, and the phenomenon of drug effects weakening or changing over time. The sixth category was “Other Drugs and Drug Combinations," which included comments about effects of drugs other than Lexapro and Seroquel, recommendations for or warnings against specific drug combinations, and lists of consumers' current and past drug regimens. The final category under "My Drug” was “Theories of Drug Action," reflecting consumers' comments about how drugs work in their brains or bodies. 
In addition to the "My Drug" umbrella category, there were 5 other smaller categories called: "My Doctor," "Finances/The System," "Family and Friends," "Pharmaceutical Companies/FDA," and "Support and Advice." The final codebook included 6 codes under "My Doctor," all of which reflected some type of interaction, conflict, or question related to doctors. The "Finances/The System" category included comments related to the expense or affordability of drugs, insurance-related issues, involuntary hospitalization and forced medication, and other comments about navigating through hospitals and "the system." All comments about family and friends fell under the appropriately named category. This included stories about a drug's effect on a family member or friend, a drug effect that a family member or friend observed in the consumer, and situational information related to having supportive or unsupportive family and friends. Codes falling under the "Pharmaceutical Companies/FDA" category included statements about the FDA approval status of a drug or indication, and remarks about the business interests of pharmaceutical companies in making and marketing drugs. Finally, comments from consumers who were worried about possible drug effects or who were seeking the experiences or advice of other users were coded under the "Support and Advice" category.

My drug: Assessing the overall experience.

Table 4.42 shows the frequency of consumers within each of the 5 websites who mentioned a code in this category. Specific to Lexapro and Seroquel, slightly more consumers described these drugs as "a miracle/saved my life" (10.9\%) than the opposite, "I hate this drug" (8.0\%). This ratio remained true across all websites, except for AskaPatient where an almost even number of consumers described the drugs both ways. 
The remaining codes in this category included comments about any drug experience, and so were not specific to Lexapro and Seroquel. Comments were also coded in this category only if there was a clear assessment or weighing of benefits and costs of a drug. Again, a slightly higher percentage of consumers across all websites described the benefits of a drug's use outweighing its costs (9.4\% versus $7.3 \%)$. However, the largest percentage of consumer comments within this category described drugs' limited helpfulness (15.3\%). Neither benefits nor costs necessarily outweighed one another, but rather the drug was "just okay," "not my wonder drug," or the drug had no discernable effect either way. Finally, "YMMV" or "your mileage may vary" was a popular slogan, especially among the consumer discussion forums, and meant that each individual is different and may have a different experience or come to a different assessment from the one being described. It was often used as a caveat so as not to discourage or overly encourage readers about trying a particular drug.

Table 4.43 provides a comparison of these codes with websites grouped according to their status as expert- or consumer-constructed. Statistical significance was reached for only 1 code, "YMMV," due to its predominance on the 2 consumer discussion forums. 
Table 4.42

Frequency of Consumers Within Each Website Who Mentioned a Code Within the Category "Assessing the Overall Experience"

\begin{tabular}{|l|c|c|c|c|c|c|}
\hline & $\begin{array}{c}\text { All } \\
\text { consumers }\end{array}$ & $\begin{array}{c}\text { Ask a } \\
\text { Patient }\end{array}$ & $\begin{array}{c}\text { Crazy } \\
\text { Meds }\end{array}$ & $\begin{array}{c}\text { Icarus } \\
\text { Project }\end{array}$ & $\begin{array}{c}\text { Rev } \\
\text { Health }\end{array}$ & WebMD \\
$(\mathrm{N}=1080)$ & $(\mathrm{N}=220)$ & $(\mathrm{N}=220)$ & $(\mathrm{N}=120)$ & $(\mathrm{N}=220)$ & $(\mathrm{N}=220)$ \\
\hline $\begin{array}{l}\text { Lexapro/Seroquel is } \\
\text { a miracle/saved my } \\
\text { life/I recommend it }\end{array}$ & $10.9 \%$ & $13.3 \%$ & $6.3 \%$ & -- & $12.5 \%$ & $11.7 \%$ \\
\hline $\begin{array}{l}\text { I hate } \\
\text { Lexapro/Seroquel/ } \\
\text { Stay away from it }\end{array}$ & $8.0 \%$ & $13.8 \%$ & $2.9 \%$ & -- & $6.3 \%$ & $9.2 \%$ \\
\hline $\begin{array}{l}\text { All in all, I am } \\
\text { positive/ Side effects } \\
\text { tolerable }\end{array}$ & $9.4 \%$ & $8.8 \%$ & $12.5 \%$ & $9.2 \%$ & $11.3 \%$ & $5.4 \%$ \\
\hline $\begin{array}{l}\text { Not worth it/ Side } \\
\text { effects too great }\end{array}$ & $7.3 \%$ & $7.5 \%$ & $9.2 \%$ & $6.7 \%$ & $8.8 \%$ & $4.2 \%$ \\
\hline $\begin{array}{l}\text { No or limited } \\
\text { helpfulness/ Not my } \\
\text { wonder drug }\end{array}$ & $15.3 \%$ & $8.8 \%$ & $20.0 \%$ & $19.2 \%$ & $20.0 \%$ & $10.4 \%$ \\
\hline $\begin{array}{l}\text { YMMV (Your } \\
\text { mileage may vary) }\end{array}$ & $7.3 \%$ & $3.3 \%$ & $14.2 \%$ & $23.3 \%$ & $2.1 \%$ & $1.7 \%$ \\
\hline
\end{tabular}

${ }^{\mathrm{a}}$ Frequencies include only comments specifically referring to Lexapro or Seroquel; excludes comments from IcarusProjet

${ }^{\mathrm{b}}$ Frequencies include comments referring to any drug; not limited to comments only about Lexapro or Seroquel 
Table 4.43

Number of Consumers Mentioning Codes under "Assessing the Overall Experience," According to Website Classification

\begin{tabular}{|l|c|c|c|c|}
\hline & $\begin{array}{c}\text { Consumer- } \\
\text { constructed } \\
(\mathrm{N}=600)\end{array}$ & $\begin{array}{c}\text { Expert- } \\
\text { constructed } \\
(\mathrm{N}=480)\end{array}$ & $\begin{array}{c}\text { Chi- } \\
\text { square }\end{array}$ & $\begin{array}{c}\mathrm{p}- \\
\text { value }\end{array}$ \\
\hline $\begin{array}{l}\text { Lexapro/Seroquel is a } \\
\text { miracle/saved my life/I } \\
\text { recommend it }\end{array}$ & $47^{\mathrm{a}}$ & 58 & 1.294 & 0.255 \\
\hline $\begin{array}{l}\text { I hate Lexapro/Seroquel/ Stay } \\
\text { away from it }\end{array}$ & $40^{\mathrm{a}}$ & 37 & 0.127 & 0.721 \\
\hline $\begin{array}{l}\text { All in all, I am positive/ Side } \\
\text { effects tolerable }\end{array}$ & $62^{\mathrm{b}}$ & 40 & 1.247 & 0.264 \\
\hline $\begin{array}{l}\text { Not worth it/ Side effects too great } \\
\text { No or limited helpfulness/ Not my } \\
\text { wonder drug }\end{array}$ & $98^{\mathrm{b}}$ & 31 & 0.935 & 0.334 \\
\hline \begin{tabular}{l} 
YMMV (Your mileage may vary) \\
\hline
\end{tabular} & $70^{\mathrm{b}}$ & 9 & 37.711 & 0.000 \\
\hline
\end{tabular}

ac'Consumer-constructed" count includes consumers from AskaPatient and CrazyMeds $(\mathrm{n}=480)$

b،Consumer-constructed" count includes consumers from AskaPatient, CrazyMeds, and IcarusProject

My drug: Dealing with side effects/finding optimum effectiveness.

Table 4.44 lists the frequencies of consumers who mentioned a code within the category "Dealing with Side Effects/Finding Optimum Effectiveness." The top 4 strategies mentioned by the most total consumers were: wait to see if the side effect eventually goes away on its own (19.2\%), stop taking the drug and/or switch to another drug (18.4\%), 
adjust the dosage of the drug (13.7\%), and add another drug to counteract the side effect or to enhance the effectiveness of the current drug (10.4\%).

Users of CrazyMeds mentioned each of these strategies more often than users of each of the other websites. While over $25 \%$ of users on CrazyMeds commented about or suggested adding another drug to a regimen, less than half of that percentage on any of the other 4 websites made similar comments or suggestions. In a similar pattern, CrazyMeds users commented more about mixing caffeine and alcohol with drugs, checking and monitoring blood levels, effects of and strategies for diet and exercise, and learning to simply accept and live with certain undesirable drug effects.

When websites were grouped according to their status as expert- or consumerconstructed, 4 of the 11 strategies listed in Table 4.45 were statistically significantly different between groups (Table 4.45). All 4 were more often mentioned on consumerconstructed sites than expert-constructed, though the difference for "add another med" was largely due to the predominance of that code on CrazyMeds. Users of the 3 consumer-constructed sites more often commented on changes in diet and exercise to counteract side effects or enhance drug effectiveness, and the importance of starting on a low dose of a drug and titrating slowly up to the target dose. Few users on AskaPatient described "other methods," so the statistically significant difference between groups is due to the more frequent use of this code on CrazyMeds and IcarusProject. Users of these discussion forums described such "other" strategies as crushing, cutting and scraping the coating of off pills to counteract over-sensitivities to some drugs, using Vaseline and other accessories to counteract certain sexual drug effects, and having friends or family nearby to talk them through a particularly intense mental or mood drug effect. 
Table 4.44

Frequency of Consumers Within Each Website Who Mentioned a Code Within the Category "Dealing with Side Effects/Finding Optimum Effectiveness," Mentioned by at Least 3\% of Consumers on Any Website

\begin{tabular}{|c|c|c|c|c|c|c|}
\hline & $\begin{array}{c}\text { All } \\
\text { consumers } \\
(\mathrm{N}=1080)\end{array}$ & $\begin{array}{c}\text { Ask a } \\
\text { Patient } \\
(\mathrm{N}=220)\end{array}$ & $\begin{array}{c}\text { Crazy } \\
\text { Meds } \\
(\mathrm{N}=220)\end{array}$ & $\begin{array}{l}\text { Icarus } \\
\text { Project } \\
(\mathrm{N}=120)\end{array}$ & $\begin{array}{c}\text { Rev } \\
\text { Health } \\
(\mathrm{N}=220)\end{array}$ & $\begin{array}{l}\text { WebMD } \\
(\mathrm{N}=220)\end{array}$ \\
\hline Add another med & $10.4 \%$ & $2.9 \%$ & $25.4 \%$ & $9.2 \%$ & $10.4 \%$ & $3.3 \%$ \\
\hline $\begin{array}{l}\text { Avoid / Drink } \\
\text { more } \\
\text { caffeine/alcohol }\end{array}$ & $1.5 \%$ & $1.7 \%$ & $4.6 \%$ & $0.0 \%$ & $0.4 \%$ & $0.0 \%$ \\
\hline $\begin{array}{l}\text { Changing time of } \\
\text { day }\end{array}$ & $6.3 \%$ & $4.6 \%$ & $10.8 \%$ & $1.7 \%$ & $8.8 \%$ & $3.3 \%$ \\
\hline Check blood levels & $1.4 \%$ & $0.0 \%$ & $4.2 \%$ & $2.5 \%$ & $0.4 \%$ & $0.4 \%$ \\
\hline $\begin{array}{l}\text { Diet / Food / } \\
\text { Exercise }\end{array}$ & $7.6 \%$ & $7.9 \%$ & $14.6 \%$ & $7.5 \%$ & $3.3 \%$ & $4.6 \%$ \\
\hline Dose adjustment & $13.7 \%$ & $12.5 \%$ & $20.8 \%$ & $5.0 \%$ & $16.7 \%$ & $9.2 \%$ \\
\hline $\begin{array}{l}\text { Learn to live with } \\
\text { it }\end{array}$ & $1.6 \%$ & $0.0 \%$ & $4.2 \%$ & $1.7 \%$ & $2.1 \%$ & $0.0 \%$ \\
\hline Other methods & $4.4 \%$ & $0.4 \%$ & $11.7 \%$ & $6.7 \%$ & $3.3 \%$ & $0.8 \%$ \\
\hline $\begin{array}{l}\text { Ride out the storm } \\
\text { / Eventually goes } \\
\text { away / Waiting }\end{array}$ & $19.2 \%$ & $14.2 \%$ & $27.1 \%$ & $18.3 \%$ & $25.8 \%$ & $10.0 \%$ \\
\hline $\begin{array}{l}\text { Switch meds / Stop } \\
\text { med }\end{array}$ & $18.4 \%$ & $14.6 \%$ & $29.2 \%$ & $11.7 \%$ & $19.6 \%$ & $13.8 \%$ \\
\hline $\begin{array}{l}\text { Taper SLOWLY / } \\
\text { Low starting dose }\end{array}$ & $6.8 \%$ & $7.1 \%$ & $10.8 \%$ & $7.5 \%$ & $5.4 \%$ & $3.3 \%$ \\
\hline
\end{tabular}


Table 4.45

Codes Under "Dealing with Side Effects/Finding Optimum Effectiveness" that Reached Statistical Significance $(p<.05)$ According to Website Classification

\begin{tabular}{|l|c|c|c|c|}
\hline & $\begin{array}{c}\text { Consumer- } \\
\text { constructed } \\
(\mathrm{N}=600)\end{array}$ & $\begin{array}{c}\text { Expert- } \\
\text { constructed } \\
(\mathrm{N}=480)\end{array}$ & $\begin{array}{c}\text { Chi- } \\
\text { square }\end{array}$ & $\begin{array}{c}\mathrm{p}- \\
\text { value }\end{array}$ \\
\hline Add another med & 79 & 33 & 11.357 & 0.001 \\
\hline Diet / Food / Exercise & 63 & 19 & 16.265 & 0.000 \\
\hline Other methods & 37 & 10 & 10.682 & 0.001 \\
\hline $\begin{array}{l}\text { Taper SLOWLY / Low } \\
\text { starting dose }\end{array}$ & 52 & 21 & 7.793 & 0.005 \\
\hline
\end{tabular}

My drug: Do I need meds?

Table 4.46 shows the frequency of consumers who mentioned a code under the category

“Do I Need Meds?" and Table 4.47 shows that each code reached statistical significance according to website classification ("Making a plan/Just in case..." was not included in the significance test because of empty cells). The most popular codes in this category were "Trying/tried alternative treatment" (mentioned by $8.0 \%$ of all consumers) and “Drugs won't work/I'm done/I don't need it" (7.4\%). The former code included all comments about having tried, wanting to try, or recommendations to try a non-drug treatment, such as a psychotherapy, cognitive-behavioral therapy, group therapy, holistic therapies, and electro-convulsive therapy, among others. The latter code included comments from consumers who wanted to quit drug treatment altogether, who determined they no longer needed or wanted drug treatment, or who thought drug treatment was not working to solve their particular problems. 
In contrast to the previous category where CrazyMeds dominated, the present category was dominated by users of IcarusProject. More users of IcarusProject (25.8\%) than any other website commented that they wanted to quit or did not need drug treatment, however more users of this forum (11.7\%) compared to the other sites also commented that drug treatment may be part of their long-term solution. The code "Making a plan/Just in case...," reflecting consumers' making prevention, emergency, or back-up plans, was only brought up in the 2 consumer discussion forums and not on the other websites.

Table 4.46

Frequency of Consumers Within Each Website Who Mentioned a Code Within the Category "Do I Need Meds?"

\begin{tabular}{|l|c|c|c|c|c|c|}
\hline & $\begin{array}{c}\text { All } \\
\text { consumers } \\
(\mathrm{N}=1080)\end{array}$ & $\begin{array}{c}\text { Ask a } \\
\text { Patient } \\
(\mathrm{N}=220)\end{array}$ & $\begin{array}{c}\text { Crazy } \\
\text { Meds } \\
(\mathrm{N}=220)\end{array}$ & $\begin{array}{c}\text { Icarus } \\
\text { Project } \\
(\mathrm{N}=120)\end{array}$ & $\begin{array}{c}\text { Rev } \\
\text { Health } \\
(\mathrm{N}=220)\end{array}$ & WebMD \\
$(\mathrm{N}=220)$
\end{tabular}


Table 4.47

Number of Consumers Mentioning Codes Under "Do I Need Meds?” According to Website Classification

\begin{tabular}{|l|c|c|c|c|}
\hline & $\begin{array}{c}\text { Consumer- } \\
\text { constructed } \\
(\mathrm{N}=600)\end{array}$ & $\begin{array}{c}\text { Expert- } \\
\text { constructed } \\
(\mathrm{N}=480)\end{array}$ & $\begin{array}{c}\text { Chi- } \\
\text { square }\end{array}$ & $\begin{array}{c}\text { p- } \\
\text { value }\end{array}$ \\
\hline $\begin{array}{l}\text { Drugs won't work / I'm done / I } \\
\text { don't need it }\end{array}$ & 63 & 17 & 18.825 & 0.000 \\
\hline $\begin{array}{l}\text { Going back on meds / Meds } \\
\text { may be the solution }\end{array}$ & 26 & 7 & 7.441 & 0.006 \\
\hline $\begin{array}{l}\text { Looking back on it.../Since I } \\
\text { stopped the med... }\end{array}$ & 39 & 12 & 9.483 & 0.002 \\
\hline $\begin{array}{l}\text { Trying/Tried alternative } \\
\text { treatment }\end{array}$ & 70 & 16 & 26.268 & 0.000 \\
\hline
\end{tabular}

Other "My Drug” categories.

Table 4.48 provides the frequency of consumers mentioning a code within the remaining 4 categories under "My Drug," as well as the most popular code within each category. Each of the categories and codes listed were dominated by users of the 2 consumer discussion forums. Table 4.49 shows significance testing for these codes, except for "Theories of Drug Action" because of empty cells. Under the category "Evidence," the code “drug or something else?" was mentioned by over $10 \%$ of all consumers. This code reflected the idea of uncertainty as to the nature or cause of an observed drug effect. Most often cited causes of a drug effect were the drug itself, other drugs the consumer was taking, lifestyle choices (diet, exercise, etc), a return of their mental illness, or a placebo effect. For example, in a review of Seroquel on AskaPatient, 
a consumer noted, "I do have some memory and concentration problems, but these could be related to 25 years with depression and bipolar disorder, or to the other meds I take (Wellbutrin 400mg, Depakene 400mg).” A user on CrazyMeds remarked that some people find Seroquel helpful for depression, but "Whether it's the consistent sleep or the drug itself I'm not entirely sure, but I suspect it's a combination of the two." Again referring to Seroquel, a user on IcarusProject commented, “I don't know if I gained weight from it (Seroquel). I am fat to start with, plus I was taking lithium and depressed/not exercising." Other users commented more generally on the dilemma, "it's kinda hard to tell hebee jebees from side effects, or crazyness, or drugs doing what they are supposed to do." Still other users provided a best guess as to the cause of an effect or advice, such as "try one med at a time. If you have reactions or side effects after beginning two meds at once, you won't know which to blame."

Under the category "Other Drug Issues" the codes relating to drug dependence/ withdrawal, and diagnosis/symptoms were both mentioned by $10.5 \%$ of all consumers, though very few consumers outside of the 2 discussion forums mentioned the latter. The former code included comments about drug discontinuation or withdrawal experiences, and advice about how to properly discontinue a drug to avoid adverse effects. Most users who offered advice about discontinuation suggested tapering slowly off the drug rather than stopping "cold turkey." For example, users on CrazyMeds advised "taking a very low $5 \mathrm{mg}$ (of Lexapro) every few days to keep the brain zaps away," or that "generally $10 \%$ reduction a week is a good rule of thumb." This code also included the idea of becoming "dependent" on the drug by, for example, having to take increasing amounts over time to achieve the same effect or having to remain on the drug because of 
intolerable discontinuation effects. As one user shared, "The first time I tried coming off Lexapro (earlier this year), I had anxiety and general pissed off and agitated feeling. So, I just went back to my normal dose." A user on WebMD voiced a common apprehension about Seroquel, "It really does work but I am concerned that I will never get off of it and develop sleep patterns of my own. I can't sleep a full night without it."

A full $25 \%$ of all consumers commented on or described their current drug cocktail or their previous drug history (code: "My drug combo/So many damn meds..."). Most of these comments came from the 2 discussion forums as well as a high percentage from RevolutionHealth. Table 4.49 shows that this was the only of the above-mentioned codes that did not achieve a significant chi-square value between expert- and consumerconstructed websites. Comments within this code included references to specific drug cocktails users have tried or are trying, as well as the general concept of having tried many different drugs in the past. A typical recounting of a drug history might resemble this comment from a CrazyMeds user, "I have tried other drugs - Zoloft, Wellbutrin, Prozac, Cymbalta. I couldn't tolerate Wellbutrin and Cymbalta. I felt numb emotionally on Zoloft. Prozac caused anorgasmia yet did nothing for my depression." More generally, users referred to the idea that they've "tried virtually every medication out there," they've been on the "med merry-go-round" for a long time, or they've "taken so many damn meds" that they have difficulty recalling which ones had what effects on them.

The final category, "Theories of Drug Action," was the least mentioned by consumers across websites, and was again dominated by users of the 2 discussion forums. This category included all descriptions of how or why drugs work in the body and brain, as well as references to drugs being "potent" or "selective" in their action on the brain. For 
example, users of CrazyMeds often attempted to explain differences in drug efficacy or the safety of a particular drug combination by referencing drugs' action in the body: "Lots of pdocs (psychiatrists) in my area are giving stims (stimulants) to people that have to take AP's (antipsychotics). In theory, the dopamine actions are occurring in different places. There are 5 dopamine pathways..." Similarly, "Celexa hits the serotonergic system less hard, which might be a pro concerning side effects." No consumers on WebMD or AskaPatient and few consumers on RevolutionHealth (1.3\%) mentioned the most common code within this category, and a chi-square test was not completed due to these zero frequencies.

In sum, all of the above "My Drug" themes were dominated by users of the 2 discussion forums. Users of CrazyMeds offered the most strategies for dealing with side effects, while users of IcarusProject commented most on the need for drugs and alternative treatments. Taken together, the "My Drug" themes showed a rich variety of concerns, experiences, and perspectives of consumers. 
Table 4.48

Frequency of Consumer Comments on Drug Theme Categories (in bold text) and the Most Frequently Mentioned Code Within that Category (in plain text)

\begin{tabular}{|l|c|c|c|c|c|c|}
\hline & $\begin{array}{c}\text { All } \\
\text { consumers } \\
(\mathrm{N}=1080)\end{array}$ & $\begin{array}{c}\text { Ask a } \\
\text { patient } \\
(\mathrm{N}=220)\end{array}$ & $\begin{array}{c}\text { Crazy } \\
\text { Meds } \\
(\mathrm{N}=220)\end{array}$ & $\begin{array}{c}\text { Icarus } \\
\text { Project } \\
(\mathrm{N}=120)\end{array}$ & $\begin{array}{c}\text { Rev } \\
\text { Health } \\
(\mathrm{N}=220)\end{array}$ & WebMD \\
Evidence & $15.3 \%$ & $9.6 \%$ & $31.3 \%$ & $29.2 \%$ & $7.9 \%$ & $5.4 \%$ \\
\hline $\begin{array}{l}\text { Drug or something } \\
\text { else? }\end{array}$ & $10.4 \%$ & $5.0 \%$ & $23.3 \%$ & $20.8 \%$ & $4.6 \%$ & $3.3 \%$ \\
\hline Other Drug Issues & $34.1 \%$ & $20.4 \%$ & $60.4 \%$ & $58.3 \%$ & $28.8 \%$ & $14.6 \%$ \\
\hline $\begin{array}{l}\text { Dependence / } \\
\text { Withdrawal }\end{array}$ & $10.5 \%$ & $5.4 \%$ & $18.8 \%$ & $24.2 \%$ & $6.3 \%$ & $4.6 \%$ \\
\hline $\begin{array}{l}\text { Diagnoses / } \\
\text { Symptoms }\end{array}$ & $10.5 \%$ & $0.8 \%$ & $22.1 \%$ & $40.0 \%$ & $3.3 \%$ & $0.8 \%$ \\
\hline $\begin{array}{l}\text { Other Drugs and } \\
\text { Drug Combinations }\end{array}$ & $32.4 \%$ & $12.5 \%$ & $51.7 \%$ & $63.3 \%$ & $32.9 \%$ & $17.1 \%$ \\
\hline $\begin{array}{l}\text { My drug combo / So } \\
\text { many damn meds... }\end{array}$ & $25.4 \%$ & $10.8 \%$ & $35.0 \%$ & $46.7 \%$ & $29.2 \%$ & $15.8 \%$ \\
\hline $\begin{array}{l}\text { Theories of Drug } \\
\text { Action }\end{array}$ & $3.9 \%$ & $0.4 \%$ & $10.8 \%$ & $9.2 \%$ & $1.7 \%$ & $0 \%$ \\
\hline meurotransmitters & $2.3 \%$ & $0 \%$ & $7.1 \%$ & $4.2 \%$ & $1.3 \%$ & $0 \%$ \\
\hline
\end{tabular}


Table 4.49

Number of Consumers Mentioning Codes under Other Drug Theme Categories According to Website Classification

\begin{tabular}{|l|c|c|c|c|}
\hline & $\begin{array}{c}\text { Consumer- } \\
\text { constructed } \\
(\mathrm{N}=600)\end{array}$ & $\begin{array}{c}\text { Expert- } \\
\text { constructed } \\
(\mathrm{N}=480)\end{array}$ & $\begin{array}{c}\text { Chi- } \\
\text { square }\end{array}$ & $\begin{array}{c}\mathrm{p}- \\
\text { value }\end{array}$ \\
\hline EVIDENCE: Drug or something else? & 93 & 19 & 38.217 & 0.000 \\
\hline $\begin{array}{l}\text { OTHER DRUG ISSUES: } \\
\text { Dependence/Withdrawal }\end{array}$ & 87 & 26 & 23.486 & 0.000 \\
\hline $\begin{array}{l}\text { OTHER DRUG ISSUES: } \\
\text { Diagnoses/Symptoms }\end{array}$ & 103 & 10 & 64.76 & 0.000 \\
\hline $\begin{array}{l}\text { OTHER DRUGS/COMBINATIONS: } \\
\text { My drug combo/So many damn meds... }\end{array}$ & 166 & 108 & 3.76 & 0.053 \\
\hline
\end{tabular}

My doctor.

Table 4.50 shows the frequency of consumers who mentioned a code within the category "My Doctor," and Table 4.51 shows significant chi-square tests for each code according to website classification as expert- or consumer-constructed. Similar to themes under "My Drug," themes under "My Doctor" were dominated by users of the 2 discussion forums. The code "talking with doc/doc explained/consult doc" was the most popular theme across each website, except for IcarusProject where the most popular theme was “I'm taking control/I'm my own best advocate." The former code included all references to having talked with the doctor, needing to consult the doctor, and information the doctor explained or decisions the doctor made about drugs or drugs' effects. For example, consumers often relayed some information their doctor explained to 
them, "My doctor says Lexapro wakes most people up but can have the opposite effect, as it started to have with me." Many consumers noted a doctor-made decision, "I was ready to switch to another SSRI...but my doctor decided to add Wellbutrin instead." This code also included suggestions to or advice about talking with the doctor, such as general statements "Consult your doctor," more directive statements "If it's akathesia, talk to your doc about ultram," or cautions to "talk to your doctor before doing anything" that might impact your medication regimen. Finally, consumers generally advised others to be open and honest when talking to the doctor about their concerns and needs, "Being lifeless, lazy, flat, zombie...is not acceptable. Tell your Doctor that you are NOT willing to just exist."

The next code, "doctor won't listen/we disagree/ I wasn't informed," was mentioned by over $10 \%$ of all consumers, including almost $30 \%$ of consumers on IcarusProject. This code reflected disagreements and conflicts between the consumer and their doctor, remarks about the doctor being wrong about a drug or drug effect, and the idea that the doctor did not support, listen to, or properly inform them about medication issues. Some consumers described a disagreement with their doctor about the appropriate course of action and made a decision to listen or not listen to the doctor's instructions, "I begged the doc to take me off this and he didn't want to so I took myself off of it." A consumer on RevolutionHealth summarized the experience of the doctor being wrong about what to expect from a drug, "Pdoc assured me that it would help me sleep. It made me excessively tired but not sleepy. In fact, I slept less which I can’t cope with.” Similarly, some consumers complained about being misinformed or poorly informed by their doctor, "my pdoc definitely down-played the effects on my memory. I had a rude 
awakening at just how much I would lose, and for how long. It makes me angry too." Many consumer comments under this code relayed feelings of not being listened to or validated by the doctor, "Is there anyone who had/has this experience with such a low dose? My Pdoc sometimes looks at me like I am making all this stuff up, I am not. I am depressed but not stupid."

The most popular "My Doctor" code among IcarusProject users and mentioned by about $9 \%$ of all consumers was "I'm taking control/I'm my own best advocate." These were comments about consumers becoming informed or advocating for themselves to the doctor, often by doing their own research on a drug, monitoring their treatment, or asserting their treatment-related decisions to their doctor. Many consumers offered general advice, such as "definitely do your own research on all things," or explained their understanding of an issue "based on my research." Some consumers requested specific drugs from their doctor or suggested that others do so, "Read about each one (medication) and pick one that you think you would like to try, actually pick a few because he's (the doctor) probably going to object to a few of them. Try one and see how it works." Also typical were consumers making treatment-related decisions on their own, such as adjusting their dose of a drug, "I also decided last week to try to decrease my Seroquel dose and see if I could sleep a little less and have more energy. I didn't ask my doctor."

The final codes under "My Doctor" mentioned by at least 3\% of all consumers were "trusting the doctor?" and "other doctor-related issues." About $8 \%$ of all consumers, and a fifth or more on each of the discussion forums, were hesitant to fully trust their doctor's word. Some consumers were seeking to verify with others some information the doctor 
had given them, such as "my psychiatrist says Lexapro is the Cadillac of SSRI's, is this really true or just a one person bias?" At other times, consumers would chime in with suspicion or caution about a doctor-made decision of another consumer, "The fact that she (the doctor) even considered lithium with your history of epilepsy means she's throwing darts.” Finally, a small percentage of consumers (4.3\%) made a comment related to "the doctor" that did not fit in the above codes, such as advice for finding a new doctor or praise for having a supportive doctor.

Similar to findings for "My Drug," the comments for "My Doctor" reflected an array of relationships and interactions with, and feelings towards doctors.

Table 4.50

Frequency of Consumers within Each Website who Mentioned a Code Within the Category "My Doctor," Mentioned by at Least 3\% of All Consumers

\begin{tabular}{|l|c|c|c|c|c|c|}
\hline & $\begin{array}{c}\text { All } \\
\text { consumers } \\
(\mathrm{N}=1080)\end{array}$ & $\begin{array}{c}\text { Ask a } \\
\text { Patient } \\
(\mathrm{N}=220)\end{array}$ & $\begin{array}{c}\text { Crazy } \\
\text { Meds } \\
(\mathrm{N}=220)\end{array}$ & $\begin{array}{c}\text { Icarus } \\
\text { Project } \\
(\mathrm{N}=120)\end{array}$ & $\begin{array}{c}\text { Rev } \\
\text { Health } \\
(\mathrm{N}=220)\end{array}$ & WebMD \\
$(\mathrm{N}=220)$
\end{tabular}


Table 4.51

Number of Consumers Mentioning Codes under "My Doctor" According to Website Classification

\begin{tabular}{|l|c|c|c|c|}
\hline & $\begin{array}{c}\text { Consumer- } \\
\text { constructed } \\
(\mathrm{N}=600)\end{array}$ & $\begin{array}{c}\text { Expert- } \\
\text { constructed } \\
(\mathrm{N}=480)\end{array}$ & $\begin{array}{c}\text { Chi- } \\
\text { square }\end{array}$ & $\begin{array}{c}\mathrm{p}- \\
\text { value }\end{array}$ \\
\hline $\begin{array}{l}\text { Talking with doc / Doc } \\
\text { explained / Consult doc }\end{array}$ & 137 & 44 & 35.703 & 0.000 \\
\hline $\begin{array}{l}\text { Doctor won't listen / We } \\
\text { disagree / I wasn't informed }\end{array}$ & 80 & 31 & 13.668 & 0.000 \\
\hline $\begin{array}{l}\text { I'm taking control / I'm my own } \\
\text { best advocate }\end{array}$ & 86 & 11 & 47.3 & 0.000 \\
\hline Trusting the doctor ? & 77 & 12 & 37.656 & 0.000 \\
\hline Other doctor-related issues & 41 & 5 & 21.935 & 0.000 \\
\hline
\end{tabular}

Finances/the system.

Table 4.52 shows the frequency of consumer comments within the remaining theme categories along with their most popular code. Under "Finances/The System," $6.5 \%$ of all consumers made a comment related to the expense of drugs and insurancerelated issues. This code appeared from consumers on all websites, though only once on AskaPatient. Many of the comments under this code were related to the cost of brand name drugs compared to generic drugs, and the involvement of insurance companies in trying to reduce costs by forcing consumers to switch to cheaper generics. A typical comment related to this was, "I have to switch from Lex to Celexa because of insurance BS and I really hope it doesn't get any worse than it is," or "The main downfall to 
Lexapro is it is another drug on the "hit list" of drugs my insurance company hates to pay for, so it costs me a fortune every month." Some consumers found the expense worth it, “it costs me $\$ 50$ for a month's supply. It's pricey, but so far, absolutely worth it," while others were simply unable to afford it, "due to loss of insurance and cost of prescription I had to stop taking it." A number of consumers found free samples given to doctors by drug companies to be an invaluable supplementary source, "I survive at least half the time on samples from pdoc because this stuff is so damn expensive."

\section{Family and friends.}

At least $6 \%$ of consumers on each website mentioned their family or friends in their drug review or discussion post. The most popular code in this category was "Drug did *this* to them/ doing what they did," which most often included descriptions of drug experiences of family members or friends. Consumers shared things like, "my ex boyfriend took it (Seroquel) to sleep and slept like the dead," "a close friend of mine developed NMS from Seroquel," or "I found out over the holidays that both my mother and aunt are on Seroquel too and it seems to be really helping them (both BP like me)." Often the experiences of family or friends influenced the consumer's perspective or decision on taking the drug, such as in the latter comment where the consumer was encouraged to try Seroquel based on the positive experience of her family. Others shared the negative experiences of family or friends in order to warn others of possible dangers, "My husband was in a study sponsored by Lilly using large doses of Seroquel, 800mg a day. Within 6 months he had full blown type 2 diabetes...no blood sugar issues in the 34 years prior to this. Bad, bad, bad Seroquel!" 


\section{Pharmaceutical companies/FDA.}

Under "Pharmaceutical Companies/FDA" the code "it's a business" included any remark about pharmaceutical companies being in the business of selling drugs. No consumers on the expert-constructed sites and only 1 consumer on AskaPatient made a comment that fell under this code. Many comments centered around the relationship between pharmaceutical company representatives and doctors, "my doctor must have been getting some great perks from the drug company as he kept trying to push it on me," or "I hate to piss off my regular doc, but I'm afraid she might be basing my treatment on what the Lexapro rep with all the samples told her." Consumers also made references to "drug company hype," "financial reasons NOT to do a study," "pharmacomafia," and a number of comments about the financial incentives to develop, market, and provide samples for brand name drugs versus generic drugs. "Other" pharmaceutical company issues, mentioned by $2.5 \%$ of all consumers, largely involved miscellaneous comments about lawsuits against drug companies, questions concerning "who's watching" the pharmaceutical industry, and views about the "medical model."

\section{Support and advice.}

Nearly $15 \%$ of all consumers elicited others' experiences of drugs, or described being scared or worried about taking a drug, usually after reading of others' experiences on the Internet. In a review of Lexapro, a consumer on AskaPatient remarked they were "rather scared of going off (of Lexapro) after reading about it on this site, but think that's the only solution at this point," and another user on the site was worried because, "I am quite health conscious and read some reviews where people put on a lot of weight." Many consumers were seeking advice from others with first-hand experience, "Has anyone had 
experience with generic Lex? Anyone switched from the name brand to the generic?" Another popular question related to Lexapro was, "Do sexual side effects ever wear off?" In general, though, most questions shared some common themes: "Is this normal?" "Has anyone else experienced this?" "Do you think this drug has caused/will cause this effect?" and "Will this go away?" 
Table 4.52

Frequency of Consumer Comments on Other Theme Categories (in bold text) and the Most Frequently Mentioned Code Within that Category (in plain text)

\begin{tabular}{|l|c|c|c|c|c|c|}
\hline & $\begin{array}{c}\text { All } \\
\text { consumers } \\
(\mathrm{N}=1080)\end{array}$ & $\begin{array}{c}\text { Ask a } \\
\text { patient } \\
(\mathrm{N}=220)\end{array}$ & $\begin{array}{c}\text { Crazy } \\
\text { Meds } \\
(\mathrm{N}=220)\end{array}$ & $\begin{array}{c}\text { Icarus } \\
\text { Project } \\
(\mathrm{N}=120)\end{array}$ & $\begin{array}{c}\text { Rev } \\
\text { Health } \\
(\mathrm{N}=220)\end{array}$ & WebMD \\
\hline $\begin{array}{l}\text { Finances/The } \\
\text { System }\end{array}$ & $8.1 \%$ & $0.8 \%$ & $14.2 \%$ & $27.5 \%$ & $5.0 \%$ & $2.9 \%$ \\
\hline $\begin{array}{l}\text { Expenses/Insurance } \\
\text { Friends and Family }\end{array}$ & $11.3 \%$ & $6.7 \%$ & $12.9 \%$ & $31.7 \%$ & $7.9 \%$ & $7.5 \%$ \\
\hline $\begin{array}{l}\text { Drug did *this* to } \\
\text { them/ Doing what } \\
\text { they did }\end{array}$ & $5.0 \%$ & $2.1 \%$ & $7.5 \%$ & $14.2 \%$ & $2.9 \%$ & $2.9 \%$ \\
\hline $\begin{array}{l}\text { Pharmaceutical } \\
\text { Companies/FDA }\end{array}$ & $6.1 \%$ & $0.4 \%$ & $13.3 \%$ & $23.3 \%$ & $1.3 \%$ & $0.8 \%$ \\
\hline $\begin{array}{l}\text { It's a business } \\
\text { Other pharma/ } \\
\text { regulatory/FDA } \\
\text { issues }\end{array}$ & $2.6 \%$ & $0.4 \%$ & $6.3 \%$ & $10.0 \%$ & $0.0 \%$ & $0.0 \%$ \\
\hline $\begin{array}{l}\text { Support and } \\
\text { Advice }\end{array}$ & $16.9 \%$ & $6.3 \%$ & $34.2 \%$ & $38.3 \%$ & $8.3 \%$ & $8.3 \%$ \\
\hline $\begin{array}{l}\text { Seeking experiences } \\
\text { effects }\end{array}$ & $14.5 \%$ & $4.2 \%$ & $32.1 \%$ & $27.5 \%$ & $7.5 \%$ & $7.9 \%$ \\
\hline
\end{tabular}


Themes by Experts versus Consumers

Overview of expert themes.

Expert text included codes from each of the 6 main thematic categories reviewed above for consumers (My Drug, My Doctor, Finances/The System, Family and Friends, Pharmaceutical Companies/FDA, and Support and Advice). However, from consumer text, there were 61 codes across the 6 categories. Table 4.53 lists the 28 of these 61 codes (45.9\%) that were mentioned in the expert text. There remained 33 themes mentioned by consumers from the 6 categories that did not come up in the expert text. Of the 28 themes mentioned, 4 of them came up in all 4 expert cases: talking with doc/doc explained/consult doc, dose-related issues, other drug issues, and targets/restores brain chemicals. Sixteen of the 28 codes (57\%) came up on one expert site and not the other.

The 4 above-stated codes mentioned by all expert cases were also the most frequently mentioned codes in expert text (there were multiple mentions of each code in a single case). The code "talking with doc..." was mentioned in these 4 cases a total of 109 times. As a proportion of total mentions of all themes, this meant that $52.7 \%$ of all mentions of theme codes by experts were related to "talking with doc/doc explained/consult doc." In contrast, a total of 181 consumers mentioned this theme 316 times, so that $5.1 \%$ of all mentions of theme codes by consumers were related to "talking with doc..." To ensure a fair comparison, the same proportion was taken for consumers using total mentions of only the 28 themes in expert text (rather than dividing the 316 mentions by total mentions of all 61 themes), and $11.2 \%$ of all mentions by consumers out of those 28 themes were related to "talking with doc..." 
Figure 4.11 compares experts and consumers on the proportion of all thematic mentions according to the most popular themes of each group (note: "talking with doc..." is not included on this table). The second most popular theme in expert text was issues related to dosing, such as what to do in the case of a missed dose or overdose, as well as instructions to take the drug as prescribed and with food and water. Next were "Other" drug issues, which were largely related to proper storage of the drug, for example, "do not refrigerate." Two of the 4 most popular themes of consumers did not show up in expert text: "My drug combo/so many damn meds" and "No/limited helpfulness/not my wonder drug."

Table 4.53

Codes From All Theme Categories That Were Mentioned in Expert Text

\begin{tabular}{|l|c|c|}
\hline & RevolutionHealth (N=2) & WebMD (N=2) \\
\hline OTHER drug issues & 2 & 2 \\
\hline $\begin{array}{l}\text { Talking with doc / Doc } \\
\text { explained / Consult doc }\end{array}$ & 2 & 2 \\
\hline $\begin{array}{l}\text { Targets/Restores brain } \\
\text { chemicals }\end{array}$ & 2 & 2 \\
\hline $\begin{array}{l}\text { Dose related issues / advice } \\
\text { Dependence / Withdrawal }\end{array}$ & 1 & 2 \\
\hline $\begin{array}{l}\text { Check blood levels } \\
\text { Eventually goes } \\
\text { away/Waiting }\end{array}$ & 1 & 2 \\
\hline Other pharma/FDA issues & 1 & 2 \\
\hline
\end{tabular}




\begin{tabular}{|l|c|c|}
\hline & RevolutionHealth (N=2) & WebMD (N=2) \\
\hline YMMV & 1 & 2 \\
\hline FDA approval status & 2 & \\
\hline $\begin{array}{l}\text { Family/friend observed an } \\
\text { effect }\end{array}$ & 2 & \\
\hline Expense / Insurance & 2 & \\
\hline $\begin{array}{l}\text { Generic/other drug } \\
\text { equivalence }\end{array}$ & 1 & \\
\hline Dose adjustment & 1 & \\
\hline $\begin{array}{l}\text { Other methods (to deal with } \\
\text { side effects) }\end{array}$ & 1 & \\
\hline $\begin{array}{l}\text { Efficacy/Safety not } \\
\text { established }\end{array}$ & 1 & 2 \\
\hline $\begin{array}{l}\text { All in All, I am positive / } \\
\text { Side effects tolerable }\end{array}$ & 1 & 2 \\
\hline Switch meds / Stop med & & \\
\hline $\begin{array}{l}\text { Taper SLOWLY / Low } \\
\text { starting dose }\end{array}$ & & \\
\hline Other doctor-related issues \\
\hline $\begin{array}{l}\text { Don't take this drug/ } \\
\text { combination }\end{array}$ & 1 & \\
\hline $\begin{array}{l}\text { Possible effects / What to } \\
\text { expect from other drugs }\end{array}$ & & \\
\hline $\begin{array}{l}\text { Avoid / Drink more } \\
\text { caffeine/alcohol }\end{array}$ & & \\
\hline Other general support & & \\
\hline
\end{tabular}




\begin{tabular}{|l|c|c|}
\hline & RevolutionHealth (N=2) & WebMD (N=2) \\
\hline $\begin{array}{l}\text { I'm taking control / I'm my } \\
\text { own best advocate }\end{array}$ & & 1 \\
\hline Diet / Food / Exercise & & 1 \\
\hline Routine & & 1 \\
\hline
\end{tabular}

Figure 4.11 Proportion of Mentions of the Most Popular Themes by Experts and Consumers, Respectively, Out of Total Mentions of All Themes ${ }^{\mathrm{a}}$

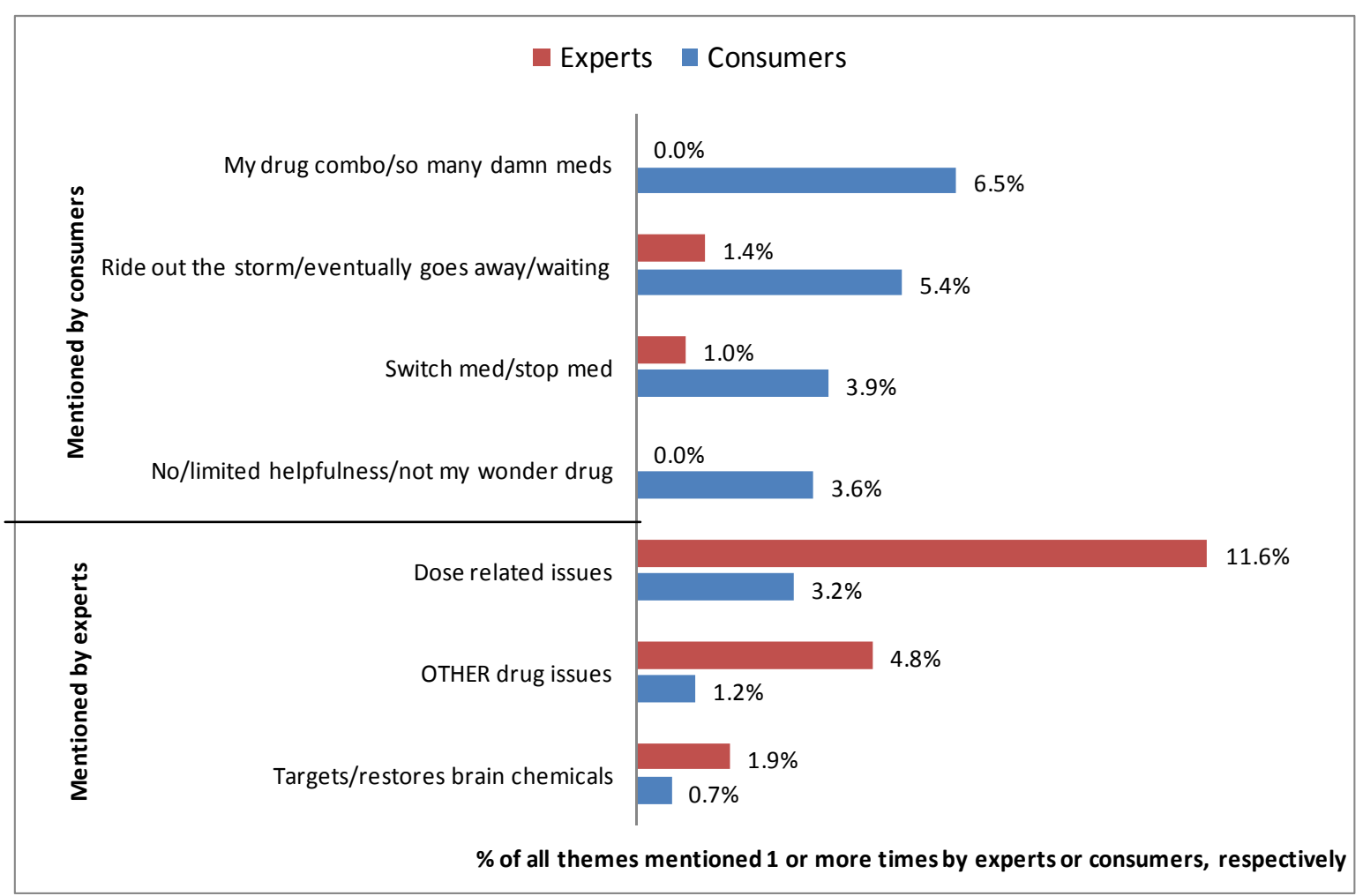

${ }^{a}$ There may be more than 1 mention in a single case. As an example, 274 consumers mentioned "My drug combo/so many damn meds" a total of 400 times. The code frequency (400) divided by the total number of mentions of all 60 themes equals $6.5 \%$. 
Assessing the overall experience.

Table 4.54 compares consumers and experts on codes within "assessing the overall experience." Just over $15 \%$ of consumers made an assessment of the benefits and costs of a drug experience and decided that the drug was of limited benefit or that it was "just okay." Another $9.4 \%$ of consumers decided that the drug benefits outweighed costs, and $7.3 \%$ decided that costs outweighed benefits. In expert text, only the "benefits outweigh costs" code appeared, while the other 2 codes were absent. WebMD mentioned this code for each drug under study (see exact statement in Table 4.55), while no codes under this category appeared in the expert text of RevolutionHealth. Table 4.55 provides excerpts that illustrate each position.

Table 4.54

Number of Consumer and Expert Cases that Mention a Code under "Assessing the Overall Experience"

\begin{tabular}{|l|c|c|}
\hline & $\begin{array}{c}\text { Consumers } \\
\mathrm{N}=1080 \\
\mathrm{~N}(\%)\end{array}$ & $\begin{array}{c}\text { Experts } \\
\mathrm{N}=4 \\
\mathrm{~N}(\%)\end{array}$ \\
\hline All in all, it is worth it (Benefits outweigh costs) & $102(9.4 \%)$ & $2(50 \%)$ \\
\hline Side effects are too great (Costs outweigh benefits) & $79(7.3 \%)$ & 0 \\
\hline No or limited helpfulness & $165(15.3 \%)$ & 0 \\
\hline
\end{tabular}


Table 4.55

Expert and Consumer Descriptions of "Assessing the Overall Experience"

\section{All in all, it is worth it}

EXPERT: "Remember that your doctor has prescribed this medication to you because he or she has judged that the benefit to you is greater than the risk of side effects" (WebMD)

\section{CONSUMER:}

"I have almost no sex drive... a dry mouth most of the time... (and) have gained some weight. But as long as I don't have to deal with the panic attacks I am ok.” (\#445)

"If you gain weight on this drug, I have found it is very hard to shed it off while still on it. But, it is so much better to be bootylicious than suicidal" (\#138)

"I wake up and feel a little doped out in the mornings however I can live with that considering it's saved me from the tragedy of no sleep and late night TV!" (\#572)

\section{Side effects are too great}

\section{EXPERT: N/A}

\section{CONSUMER:}

"Lexapro did not work for me. I felt distant, with no emotions. No, I wasn't depressed anymore but I'm not sure the side effects were worth it!" (\#34)

"Felt like a new man for 5 months. Best most secure days of my life. I quit - you guessed it - NO sex drive, maybe one erection a week and impossible to orgasm.” (\#433)

"Gained 30 pounds in seven months...enough weight gain that I am going off this drug even though it works" (\#571)

\section{No or limited helpfulness}

EXPERT: N/A 


\section{CONSUMER:}

"Helped depression, but caused weight gain, which caused more depression... a vicious cycle." (\#11)

"Lexapro sucked. My doctor prescribed lexapro and it didn't make me feel any better." (\#263)

"I was prescribed Seroquel, and I do have to say that it did bring me back to a more stable state at the time. That state was not exactly anything to write home about, but at least it was an improvement over where I was before." (\#650)

"Not the perfect med but better than others I have tried...less side effects too." (\#920)

\section{Dealing with side effects/finding optimum effectiveness.}

Figure 4.12 shows the proportion of mentions of the most popular codes of experts and consumers within the category "dealing with side effects/finding optimum effectiveness" out of total mentions of all codes within that category. Only 1 code, "Add another med," was mentioned by one group (consumers) and not the other (experts). The most frequently mentioned code among experts ( $24 \%$ of mentions) was "other methods," which primarily included cautions to stand up slowly to avoid dizziness and to take care when driving a vehicle. Consumers who mentioned this code provided similar advice, as well as offered additional recommendations, such as allow plenty of time for sleep at night, set multiple alarm clocks, and take a shower in the morning to help wake up.

The most frequently mentioned code among consumers ( $24 \%$ of mentions) was "ride out the storm/eventually goes away/waiting," which also took $12 \%$ of all mentions within this category in the expert text. Expert text stated that it may take " 4 weeks or longer" for Lexapro and "several weeks" for Seroquel before feeling the "full benefits" of 
the drug. Consumers made similar statements, "they take a few weeks to kick in," or "took a few weeks to get used to, excellent results after that!" While expert text uniformly listed a 4 or "several" week time frame, there was a wider range of time frames cited by consumers, from "it took about 1 week to really kick in," to "after 18 months, my body is well-adjusted to it and it helps a great deal with regulating my mood." Both of the latter statements were from reviews of Lexapro. Unlike expert text which only mentioned a time frame for benefits to be felt, consumers also shared information on how long it took for "side effects" to go away. Some consumers offered general rules of thumb, “initial side effects should go away within a week or two. If they don't, they're there to stay," and others shared their personal experience of side effects, "Depressed and zoned out bad first couple of weeks. Sexual side effects went away after 2-3 months.” While expert text did not comment on the popular consumer code "add another med," consumers infrequently mentioned the popular expert code "check blood levels." Expert text emphasized the importance of regular monitoring of blood sugar levels while on Seroquel, and periodic monitoring of other laboratory tests on either drug. Consumers offered the same information and encouragement, however, at a lower rate of mention compared to the frequencies of other codes in this category. 
Figure 4.12 Proportion of Mentions of the Most Popular Codes Within the Category "Dealing with Side Effects/Finding Optimum Effectiveness" Out of Total Mentions Within the Category

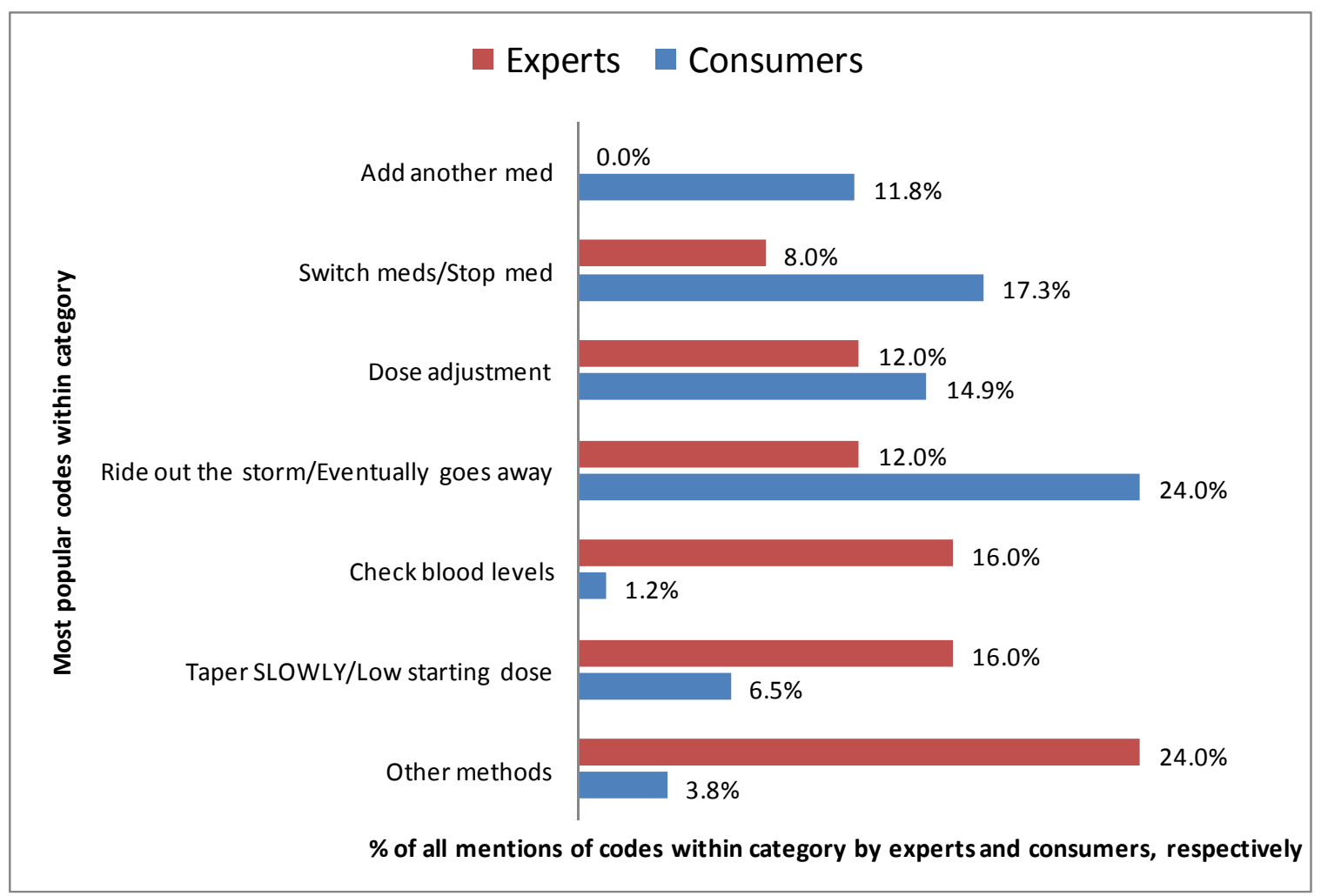

My doctor.

The code "talking with doc..." was the most frequent code found in expert text as it appeared 109 times in those 4 cases, and $18.4 \%$ of words from the total text of experts were highlighted with that code (see Table 4.56). Table 4.57 provides excerpts by experts and consumers from codes in "My Doctor." Expert text had a uniform discourse around "talking with the doc...," always pointing to the doctor as the major source for information, expertise, and guidance. Consumers also pointed to doctors as a major source for those things with remarks such as "as usual, talk to your doctor" and "I would 
recommend trying it if your doctor suggests it." Beyond this, however, consumers demonstrated a broader range of relationships with the doctor by frequently commenting on how their doctor was wrong, their doctor did not inform them of certain potential effects, their doctor didn't believe them when they shared a particular drug experience, and so on as Table 4.57 illustrates.

Table 4.56

Consumers and Experts on the "My Doctor" Code: "Talking with Doc/Doc Explained/Consult Doc"

\begin{tabular}{|l|c|c|}
\hline & Consumers & Experts \\
\hline Sample size & 1080 & 4 \\
\hline$\%$ of cases that mention the code & $16.8 \%(\mathrm{n}=181)$ & $100 \%$ \\
\hline Total number of occurrences of the code & 316 & 109 \\
\hline$\%$ of words coded out of total text & $3.8 \%$ & $18.4 \%$ \\
\hline
\end{tabular}


Table 4.57

Excerpts from Consumers and Experts on "My Doctor"

\begin{tabular}{|c|c|}
\hline \multicolumn{2}{|r|}{$\begin{array}{c}\text { Codes: Talking with doc / Doc explained / Consult doc } \\
\text { Doc was wrong / We disagree / I wasn't informed } \\
\text { Trusting Doc? }\end{array}$} \\
\hline Expert text & $\begin{array}{l}\text { "Tell your doctor about..."; "Tell your doctor if..." } \\
\text { Talk to your doctor before..." } \\
\text { Call your doctor at once if..."; "Tell the doctor immediately if..." } \\
\text { "Do not...without telling your doctor"; "Do not... without first } \\
\text { talking to your doctor" } \\
\text { "Your doctor will need to check you at regular visits..." } \\
\text { "Consult your doctor or pharmacist for more details"; } \\
\text { "Contact your doctor for..." } \\
\text { "...unless your doctor instructs you otherwise" } \\
\text { "Discuss the risks and benefits with your doctor" }\end{array}$ \\
\hline AskaPatient & $\begin{array}{l}\text { "I was told by my Psychiatrist that the only side effects were } \\
\text { sleepiness and POSSIBLE weight gain. I was not warned of the } \\
\text { diabetes risk or other side effects" (\#494) } \\
\text { "I'm not sure that the tingling that I'm feeling is from Anxiety like } \\
\text { my psychiatrist says, or as a side effect of the Lexapro. I think it's } \\
\text { the lexapro" (\#58) } \\
\text { "my doctor must have been getting some great perks from the drug } \\
\text { company as he kept trying to push it on me despite my repeated } \\
\text { telling him how awful it was" (\#16) }\end{array}$ \\
\hline CrazyMeds & $\begin{array}{l}\text { "I see Dr on the 6th - but he is fast and furious in his visits and I } \\
\text { would like input from the "experts" as well as him. I do take his } \\
\text { advise but also like other input" (\#217) } \\
\text { "I've actually read that weight loss was possible and my doctor } \\
\text { made it sound like side effects in general are rare and minor. It's } \\
\text { pretty confusing." (\#132) }\end{array}$ \\
\hline
\end{tabular}




\begin{tabular}{|c|c|}
\hline & $\begin{array}{l}\text { "Only doctors can diagnose and treat an illness. Some doctors tend } \\
\text { to get pissed off by patients who know too much about medications, } \\
\text { so tread lightly when and where appropriate." (\#1083) } \\
\text { "Some psychiatrists are really stupid." (\#638) } \\
\text { "All docs I've had, from gp to pdoc2, are obsessed with Cymbalta as } \\
\text { a pain aid. Only pdoc1 listened to me when I told her it didn't touch } \\
\text { my pain,... Pdoc2 is totally fixated on Cymbalta for pain. They all } \\
\text { have the same mantra: 'It's been tested for pain and it works."” } \\
\text { (\#685) }\end{array}$ \\
\hline RevolutionHealth & $\begin{array}{l}\text { "I chose this medication over Paxil because my doctor told me it } \\
\text { would be faster acting and have less side effects." (\#298) } \\
\text { "I think I might stay on it just long enough to show up in my pdoc's } \\
\text { office in this "condition" so that he can see first hand what I'm } \\
\text { talking about." (\#768) } \\
\text { "I've told the doc this and he says it will get better, but it's been } \\
\text { about } 2 \text { months and I am still completely incoherent in the morning." } \\
\text { (\#791) }\end{array}$ \\
\hline WebMD & $\begin{array}{l}\text { "It took my new doctor a while to find the right dose for me... It } \\
\text { took may psychiatrists to finally figure it out. None of them } \\
\text { completely understood all the past meds I was on and the weight } \\
\text { gain I experienced on all of them.” (\#959) } \\
\text { "....my whole body would jump and twitch... I was scared to drive } \\
\text { my car fear that I would cause an accident. My doctor tells me that } \\
\text { its not because of seroquel but I disagree.” (\#841) } \\
\text { “...it makes me extremely sleepy so I am not sure why she has } \\
\text { prescribed this medication twice a day. I've spoken to her numerous } \\
\text { times about this issue because it is affecting me staying awake at my } \\
\text { job..." (\#866) }\end{array}$ \\
\hline Icarus & $\begin{array}{l}\text { "People need to feel empowered with education and choice before } \\
\text { taking stuff (and don't necessarily trust the docs to tell you } \\
\text { everything you need to know)." (\#986) } \\
\text { "I finally got some appropriate drugs after the TENTH psychiatrist } \\
\text { I've seen in my life. (patience is key when looking for the right }\end{array}$ \\
\hline
\end{tabular}




\begin{tabular}{|l|l|}
\hline $\begin{array}{l}\text { drugs and the right person who actually bothers to keep up on the } \\
\text { current research and actually bothers to listen to you as an } \\
\text { individual...)" (\#962) }\end{array}$ \\
$\begin{array}{l}\text { "I argued with my doc because I had no life. All I did was sleep. She } \\
\text { was more concerned with me being stable, but the side effects were } \\
\text { so bad." (\#991) }\end{array}$ \\
\hline
\end{tabular}

\section{Dose-related issues.}

"Dose-related issues" made up 11.6\% of all mentions of themes in expert text, as Figure 4.11 shows, compared to $3.2 \%$ of all mentions of themes by consumers. Under this code, experts provided information on what to do in the case of an overdose, "seek emergency medical attention," or a missed dose, "take it as soon as you remember...(or)...skip the missed dose and resume your usual dosing schedule." Experts further emphasized to take only the prescribed dose as instructed by the doctor and to "not start, stop, or change the dosage of any medicine before checking with your doctor or pharmacist first."

Consumer comments under this code largely involved attempts to ascertain the lowest and highest appropriate and/or effective doses, "For Lexapro, 20mg is about the ceiling, though some people get doses as high as $40 \mathrm{mg}$... It would be a good idea to stick to $10 \mathrm{mg}$ until you're able to evaluate the effectivity of the drug..." A paradoxical dosing guideline for Seroquel came up several times, "Seroquel is highly sedating in the lower doses (i.e., 25, 50, 100, etc)," while "higher doses can be used for treatment of acute mania" including psychosis and bipolar mania, but not for insomnia. Rather than stating a general rule, many consumers simply shared with others their personal experience at various doses, "seroquel at low dose 50 knocks me out, 100 tired for about an hour, 200 at bed nothing, that's just me." Some consumers spoke of the "right dosage," or "correct 
dosage," noting that there is no single dose that works for everyone or for all problems, but that "once you find what it is you will know it." Other comments that appeared in consumer text included the comparability of extended release versions of drugs to regular dosing, and the possibility of using a drug PRN, or "as needed," instead of on a daily schedule.

In sum, expert text included less than half of the themes mentioned by consumers, and a majority of these themes were mentioned on only 1 or the other expert site. Expert text offered unitary discourses around benefits versus costs of the drug, and the doctor. Expert and consumer text shared several similar strategies for dealing with side effects, but consumers provided many additional practical strategies not covered in expert text. Comments about dosing issues were divergent between groups, with consumers making more specific dosing inquiries and suggestions and expert text making general references to seeking professional assistance for many dosing issues.

Usability of Consumer Sites According to "Typical" Web Use

Lexapro.

Tables 4.58 and 4.59 compare the most recent 20 posts from AskaPatient, CrazyMeds, WebMD, and RevolutionHealth $(\mathrm{n}=80)$ to the full dataset of consumer reviews for Lexapro $(\mathrm{n}=480)$. Table 4.58 compares the groups on the effects most mentioned by consumers, and shows considerable comparability for these 9 effects. One effect, extreme sleepiness/tired, reached a significant chi-square value (chi-square $=4.026$, $\mathrm{df}=1, \mathrm{p}=0.045$ ), with more consumers reporting this effect in recent posts. Overall for the most popularly mentioned effects of Lexapro, the 20 most recent posts from each website demonstrated comparable frequencies to the full dataset of consumer reviews. 
Table 4.59 makes a similar comparison for less frequently mentioned effects of Lexapro. There were 5 effects mentioned by greater than $3 \%$ of consumers in the full dataset, but mentioned by fewer than 3\% of consumers in recent posts. All 5 were physical effects, such as dizziness, sweating, and weight loss. Internet users relying on the first 20 posts on these websites run the risk of missing or underestimating these few effects. All other effects mentioned by more than $3 \%$ of consumers in the full dataset were also mentioned by at least that many in recent posts.

There were another 5 effects mentioned by few consumers $(<3 \%)$ in the full dataset that were mentioned by more consumers ( $>3 \%)$ in recent posts. Two of these were mental or mood effects: suicidal thinking/planning/attempts and crying spells/emotional rollercoaster. The full dataset contained 3 cases each of hot flash/heat stroke and vision/eye changes, all of which were present in recent posts. A typical Internet user seeing all 3 cases out of 80 , rather than out of 480 , might over-estimate the frequency of these effects. Again, though, all other effects mentioned by less than $3 \%$ of consumers in the full dataset were also mentioned by that many or fewer in recent posts.

Table 4.58 
Most Frequently Mentioned Effects of the Most Recent 20 Consumer Posts From Each Website Compared to All Consumer Posts for Lexapro

\begin{tabular}{|l|c|c|}
\hline & $\begin{array}{c}\text { Recent posts } \\
(\mathrm{N}=80)\end{array}$ & $\begin{array}{c}\text { Full dataset } \\
(\mathrm{N}=480)\end{array}$ \\
\hline Symptoms reduced / improved & $32.5 \%$ & $30.4 \%$ \\
\hline Extreme sleepiness / tired ${ }^{\mathrm{a}}$ & $32.5 \%$ & $23.8 \%$ \\
\hline Symptoms new / worsened & $18.8 \%$ & $15.8 \%$ \\
\hline Trouble sleeping / insomnia & $11.3 \%$ & $13.3 \%$ \\
\hline Emotionally stable / calm / "normal" & $15.0 \%$ & $13.1 \%$ \\
\hline Weight gain & $13.8 \%$ & $13.1 \%$ \\
\hline Nausea / sick stomach / vomiting & $11.3 \%$ & $12.1 \%$ \\
\hline Numb / detached / lack of energy or interest & $8.8 \%$ & $11.3 \%$ \\
\hline Brain fog / zombie & $12.5 \%$ & $10.8 \%$ \\
\hline
\end{tabular}

${ }^{\mathrm{a}}$ Chi-square $=4.026, \mathrm{df}=1, \mathrm{p}=0.045$

Table 4.59 
Less Frequently Mentioned Effects from Recent Posts Compared to the Full Dataset for Lexapro

\begin{tabular}{|l|c|c|c|c|}
\hline & $\begin{array}{c}\text { Recent } \\
\text { posts } \\
(\mathrm{N}=80)\end{array}$ & $\begin{array}{c}\text { Recent } \\
\text { posts } \\
\%\end{array}$ & $\begin{array}{c}\text { Full } \\
\text { dataset } \\
(\mathrm{N}=480)\end{array}$ & $\begin{array}{c}\text { Full } \\
\text { dataset } \\
\%\end{array}$ \\
\hline $\begin{array}{l}\text { Effects mentioned by }>\mathbf{3 \%} \text { of consumers in } \\
\text { full dataset, and }<\mathbf{3 \%} \text { of consumers in most } \\
\text { recent posts }\end{array}$ & & & & \\
\hline "Sweating like a pig" & 2 & $2.5 \%$ & 28 & $7.1 \%$ \\
\hline Loss of appetite & 2 & $2.5 \%$ & 21 & $5.3 \%$ \\
\hline Weight loss & 2 & $2.5 \%$ & 21 & $5.3 \%$ \\
\hline Dizzy/lightheaded/faint & 2 & $2.5 \%$ & 20 & $5.1 \%$ \\
\hline Other misc effects & 1 & $1.3 \%$ & 19 & $4.8 \%$ \\
\hline $\begin{array}{l}\text { Effects mentioned by <3\% of consumers in } \\
\text { full dataset, and }>3 \% \text { of consumers in most } \\
\text { recent posts }\end{array}$ & & & & \\
\hline Helps me sleep & 5 & $6.3 \%$ & 9 & $2.3 \%$ \\
\hline Suicidal thinking / planning / attempts & 3 & $3.8 \%$ & 6 & $1.5 \%$ \\
\hline Crying spells / emotional rollercoaster & 3 & $3.8 \%$ & 4 & $1.0 \%$ \\
\hline Hot flashes / heat stroke & 3 & $3.8 \%$ & 3 & $0.8 \%$ \\
\hline Vision / eye changes & $3.8 \%$ & 3 & $0.8 \%$ \\
\hline
\end{tabular}

Seroquel. 
Tables 4.60 and 4.61 compare the most recent 20 posts from the 4 websites $(n=80)$ to the full dataset of consumer reviews for Seroquel $(n=480)$. Table 4.60 makes this comparison using the most frequently mentioned effects of Seroquel, and again shows good comparability for these 9 effects. One effect, brain fog/zombie, reached statistical significance $($ chi-square $=3.958, \mathrm{df}=1, \mathrm{p}=0.047$ ), with more consumers in recent posts reporting this effect. Otherwise, recent posts and the full dataset appeared to be comparable in reported frequencies of the most popular effects of Seroquel.

Table 4.61 makes a similar comparison using the less frequently mentioned effects of Seroquel. Four effects were mentioned by $3 \%$ or more of consumers in the full dataset, and by few or no consumers in recent posts. Two were mental or mood effects, and two were physical effects. In contrast, the second half of the table lists the 4 effects mentioned by few consumers $(<3 \%)$ in the full dataset and by a greater number $(>3 \%)$ in recent posts. All 4 were physical effects. For the remaining effects of Seroquel, recent posts and the full dataset were comparable.

Table 4.60 
Most Frequently Mentioned Effects of the Most Recent 20 Consumer Posts From Each Website Compared to All Consumer Posts for Seroquel

\begin{tabular}{|l|c|c|}
\hline & $\begin{array}{c}\text { Recent posts } \\
(\mathrm{N}=80)\end{array}$ & $\begin{array}{c}\text { Full dataset } \\
(\mathrm{N}=480)\end{array}$ \\
\hline Helps me sleep & $41.3 \%$ & $35.6 \%$ \\
\hline Extreme sleepiness / tired & $32.5 \%$ & $33.1 \%$ \\
\hline Symptoms reduced / improved & $28.8 \%$ & $24.8 \%$ \\
\hline Weight gain & $20.0 \%$ & $22.5 \%$ \\
\hline Brain fog / zombie ${ }^{\mathrm{a}}$ & $22.5 \%$ & $15.2 \%$ \\
\hline Increased appetite / cravings & $11.3 \%$ & $12.7 \%$ \\
\hline Symptoms new / worsened & $11.3 \%$ & $10.2 \%$ \\
\hline Abnormal movements & $8.8 \%$ & $8.8 \%$ \\
\hline Other misc effects & $8.8 \%$ & $5.0 \%$ \\
\hline
\end{tabular}

${ }^{\mathrm{a}}$ Chi-square $=3.958, \mathrm{df}=1, \mathrm{p}=0.047$

Table 4.61 
Less Frequently Mentioned Effects of Recent Posts from Each Website Compared to the Full Dataset for Seroquel

\begin{tabular}{|l|c|c|c|c|}
\hline & $\begin{array}{c}\text { Recent } \\
\text { posts } \\
(\mathrm{N}=80)\end{array}$ & $\begin{array}{c}\text { Recent } \\
\text { posts } \\
\%\end{array}$ & $\begin{array}{c}\text { Full } \\
\text { dataset } \\
(\mathrm{N}=480)\end{array}$ & $\begin{array}{c}\text { Full } \\
\text { dataset } \\
\%\end{array}$ \\
\hline $\begin{array}{l}\text { Effects mentioned by }>\mathbf{3 \%} \text { of consumers in } \\
\text { full dataset, and }<\mathbf{3 \%} \text { of consumers in most } \\
\text { recent posts }\end{array}$ & 1 & $1.3 \%$ & 21 & $5.3 \%$ \\
\hline Dry mouth / sore throat & 1 & $1.3 \%$ & 20 & $5.0 \%$ \\
\hline Dreams vivid / nightmares & 1 & $1.3 \%$ & 19 & $4.8 \%$ \\
\hline Numb / detached / lack of energy or interest & 0 & $0 \%$ & 11 & $2.8 \%$ \\
\hline Sweating like a pig & 3 & $3.8 \%$ & 10 & $2.5 \%$ \\
\hline $\begin{array}{l}\text { Effects mentioned by <3\% of consumers in } \\
\text { full dataset, and }>\mathbf{3} \% \text { of consumers in most } \\
\text { recent posts }\end{array}$ & & $5.0 \%$ & 9 & $2.3 \%$ \\
\hline Headache increased & 3 & $3.8 \%$ & 5 & $1.3 \%$ \\
\hline Heartbeat changes & 3 & $3.8 \%$ & 4 & $1.0 \%$ \\
\hline Loss of appetite & & & & \\
\hline Breathing problems / shortness of breath & & & & \\
\hline
\end{tabular}

Themes.

Table 4.62 shows the 25 most frequently mentioned themes in recent posts $(\mathrm{n}=160)$ compared to the full dataset $(\mathrm{n}=960)$ for both drugs combined. For nearly all of the listed themes, recent posts showed a higher proportion of consumers mentioning the theme compared to the full dataset, though in most of the cases the differences appeared slight. Diet/food/exercise under the "dealing with side effects..." category was the only code 
mentioned by considerably fewer consumers in recent posts. Overall, the number of consumers mentioning themes appeared to be comparable between the 2 groups.

Table 4.62

Most Frequently Mentioned Themes of Most Recent 20 Posts from Each Website Compared to the Full Dataset of Both Drugs

\begin{tabular}{|l|c|c|c|c|}
\hline \multicolumn{1}{|c|}{ MY DOCTOR } & $\begin{array}{c}\text { Recent } \\
\text { posts } \\
(\mathrm{N}=160)\end{array}$ & $\begin{array}{c}\text { Recent } \\
\text { posts } \\
\%\end{array}$ & $\begin{array}{c}\text { Full } \\
\text { dataset } \\
(\mathrm{N}=960)^{\mathrm{a}}\end{array}$ & $\begin{array}{c}\text { Full } \\
\text { dataset } \\
\%\end{array}$ \\
\hline $\begin{array}{l}\text { Doctor won't listen / we disagree / I } \\
\text { wasn't informed }\end{array}$ & 18 & $11.3 \%$ & 76 & $7.9 \%$ \\
\hline $\begin{array}{l}\text { I'm taking control / I'm my own best } \\
\text { advocate }\end{array}$ & 14 & $8.8 \%$ & 56 & $5.8 \%$ \\
\hline $\begin{array}{l}\text { Talking with doc / doc explained / } \\
\text { consult doc }\end{array}$ & 27 & $16.9 \%$ & 147 & $15.3 \%$ \\
\hline Trusting the doctor ? & 16 & $10.0 \%$ & 62 & $6.5 \%$ \\
\hline \multicolumn{1}{|c|}{ MY DRUG: Assessing the Overall } & 18 & $11.3 \%$ & 105 & $10.9 \%$ \\
\hline $\begin{array}{l}\text { A miracle / saved my life / I } \\
\text { recommend }\end{array}$ & 15 & $9.4 \%$ & 77 & $8.0 \%$ \\
\hline $\begin{array}{l}\text { I hate this drug / stay away from it } \\
\text { wolerable in All, I am positive / side effects }\end{array}$ & 22 & $13.8 \%$ & 91 & $9.5 \%$ \\
\hline $\begin{array}{l}\text { Not worth it / side effects too great } \\
\text { YMMV drug }\end{array}$ & 14 & $8.8 \%$ & $71 \%$ \\
\hline
\end{tabular}




\begin{tabular}{|c|c|c|c|c|}
\hline & $\begin{array}{c}\text { Recent } \\
\text { posts } \\
(\mathrm{N}=160)\end{array}$ & $\begin{array}{c}\text { Recent } \\
\text { posts } \\
\%\end{array}$ & $\begin{array}{c}\text { Full } \\
\text { dataset } \\
(\mathrm{N}=960)^{\mathrm{a}}\end{array}$ & $\begin{array}{c}\text { Full } \\
\text { dataset } \\
\%\end{array}$ \\
\hline \multicolumn{5}{|l|}{$\begin{array}{c}\text { MY DRUG: Dealing with Side } \\
\text { Effects/Finding Optimum Effectiveness }\end{array}$} \\
\hline Add another med & 19 & $11.9 \%$ & 101 & $10.5 \%$ \\
\hline Diet / food / exercise & 6 & $3.8 \%$ & 72 & $7.6 \%$ \\
\hline Dose adjustment & 29 & $18.1 \%$ & 142 & $14.8 \%$ \\
\hline $\begin{array}{l}\text { Ride out the storm / eventually goes } \\
\text { away / waiting }\end{array}$ & 30 & $18.8 \%$ & 185 & $19.3 \%$ \\
\hline Switch meds / stop med & 32 & $20.0 \%$ & 185 & $19.3 \%$ \\
\hline \multicolumn{5}{|l|}{ MY DRUG: remaining sub-categories } \\
\hline $\begin{array}{l}\text { Drugs won't work / I'm done / I don't } \\
\text { need it }\end{array}$ & 12 & $7.5 \%$ & 49 & $5.1 \%$ \\
\hline Trying/tried alternative treatment & 7 & $4.4 \%$ & 43 & $4.5 \%$ \\
\hline Drug or something else? & 18 & $11.3 \%$ & 87 & $9.1 \%$ \\
\hline Dependence / withdrawal & 14 & $8.8 \%$ & 84 & $8.8 \%$ \\
\hline Diagnoses / symptoms & 15 & $9.4 \%$ & 65 & $6.8 \%$ \\
\hline Dose related issues & 18 & $11.3 \%$ & 95 & $9.9 \%$ \\
\hline Generic/other drug equivalence & 15 & $9.4 \%$ & 85 & $8.9 \%$ \\
\hline My drug combo / so many damn meds... & 39 & $24.4 \%$ & 218 & $22.7 \%$ \\
\hline $\begin{array}{l}\text { Possible effects / what to expect from } \\
\text { other drugs }\end{array}$ & 17 & $10.6 \%$ & 81 & $8.4 \%$ \\
\hline \multicolumn{5}{|l|}{ SUPPORT AND ADVICE } \\
\hline $\begin{array}{l}\text { Seeking experiences / worried about } \\
\text { effects }\end{array}$ & 17 & $10.6 \%$ & 124 & $12.9 \%$ \\
\hline
\end{tabular}

${ }^{a}$ Excludes postings from IcarusProject. 


\section{Preliminary Data Checking}

\section{Communication with web 2.0 informants.}

Prior to starting the present research, $9 \mathrm{Web} 2.0$ researchers, bloggers, and webmasters were contacted with inquiries as to the state-of-the-art for identifying or preventing unauthentic accounts on sites for consumer reviews or discussion. Five responses were received, and no one was able to offer a sure method. However, a few responses were noteworthy:

The owner/moderator of a consumer-constructed discussion forum related to psychiatric medications described:

We had someone from Cephalon [a pharmaceutical company] come by. The person was easy to spot. Again I'm fine with it. It's just like science. They have to defend their positions and we have to defend ours... As we didn't agree with this person all the time they just stopped showing up... As we're good with pattern recognition we can easily spot someone from the industry by the language they use. I no longer have time to read other sites, so I don't know if they are popping up elsewhere. Given that they have set up their own sites I seriously doubt if they are going to bother with peer-to-peer sites / user generated media / whatever they are calling us this month.

In a follow-up email, the owner elaborated on the type of language an industry representative might use:

Pharm reps write somewhere between high school and college-level, they don't use too many technical terms if they can avoid them. They speak to 
effects and only mild, temporary side effects. They will mention off-label usage, but everything is one condition at a time. Drug-drug interactions are only for meds someone is currently taking. They'll defend their meds if something hasn't been $100 \%$ proven, using studies that were funded by their companies or just pulling stuff out of the PI sheets. Sometimes it's as if they copied a paragraph or two from the company's website and pasted it into a post.

The Head of Operations of an expert-constructed health site that integrates expert knowledge and consumer participation responded:

Discussions with marketing personnel at a number of pharmaceutical companies (not individual sales reps) suggest a more cautious approach. Because many social networking websites with consumer blogs contain potentially unreported serious adverse events (SAE), pharma personnel are "discouraged" from visiting these websites because if they see an unreported SAE, they would be bound to report it to the FDA. [Our website] is working in collaboration with several pharma companies to submit a citizens petition (at the request of the FDA) for guidance on responsibilities for SAE reporting for on-line communities... For [our website], we also screen our patient comments for promotional messages and anything with a promotional message is deleted... Hope that helps shed a bit of light on "official" FDA guidance, senior pharma response, and our activities. What happens at the rep level, however, is tough to monitor. That said, with $250,000+$ individuals 
enrolled in [our website], its hard to think that "sales representatives going against FDA/company policies" can skew the data significantly.

David and Pinch (2005) found that individuals contributing to product review sites may have a number of agendas apart from sharing a genuine experience or expertise. In their analysis of 50,000 user reviews of 10,000 books and CDs on amazon.com, they found that about $1 \%$ of user reviews were plagiarized either verbatim or with variations. Plagiarized reviews appeared to promote a certain agenda, carry out personal attacks, and boost the user's reputation in the amazon.com tiered reputation system, among other things. They concluded that the great majority of reviews, however, "can probably be taken at face value and are authors' attempts to give their own honest appraisal of the product” (p. 23). Both authors were contacted as part of the above inquiry, and neither was aware of a program or method to judge the authenticity of user contributions. The software program they developed to detect plagiarism on amazon.com is partly available open-source, but would not have fit the needs of the present research (and would have require considerable re-programming for use on another website).

\section{Wikiscanner search.}

Wikiscanner was used to search Astra-Zeneca, Eli Lilly, and GlaxoSmithKline for Wikipedia edits. Any edited entries related generally or specifically to medications or pharmaceutical companies were identified. A detailed listing of search results can be found in Appendix H. Briefly, there were 949 edits by persons on Astra Zeneca's network, of which $23(2.4 \%)$ were relevant to medications or drug companies. These included 1 or more edits to the following entries: AstraZeneca, Federal Food, Drug, and Cosmetic Act, FDA, Good Manufacturing Practice, Omeprazole, Quetiapine, 
Risperidone, and Thalidomide. In addition to the publicized quetiapine edit mentioned above, a sentence about the heavy marketing of omeprazole in the face of generic competition was deleted and summaries of several studies in which omeprazole outperformed its competitor drug were added. Such edits may be considered controversial or salacious as there is a very possible conflict of interest involved. However, there were only 3 such edits, equaling $13 \%$ of the 23 relevant edits and $0.3 \%$ of all 949 edits.

There were 832 edits returned for Eli Lilly, of which 38 (4.6\%) were relevant to medications or drug companies. In one edit, 6 contraindications were deleted for drotrecogin alfa, including for pregnancy, breast feeding, and use in patients under 18 years of age. A link for Lilly's drug Cialis was added to the page for erectile dysfunction. The word "deceptive" was deleted in a description of Lilly's marketing campaign for a drug. On the user talk pages for fluoxetine (where users discuss and justify edits to an entry), a person from the Eli Lilly network stated, "I have been taking fluoxetine and had very little in the way of side effects - just a small amount of nausea at the start. It has been very successful in treating my depression so far and I'm feeling good for the first time in ages..."

Finally, a search for GlaxoSmithKline returned 1,148 edits, of which 31 (2.7\%) were related to medications or the drug industry. Under the entry for "GSK," someone from their network added a section titled "Good Works" and a paragraph describing the company's global leadership in eradicating lymphatic filariasis (elephantiasis). Otherwise, Wikipedia edits from these companies appeared innocuous, even when they were related to specific medications the company had a financial interest in (see Appendix H). Of course, it was also apparent that each company did monitor entries 
relevant to their respective company or a specific drug they marketed, as a majority of relevant edits were done to their own company and drugs.

In sum, it appears that verifying whether users are authentically representing themselves on the Web is an elusive quest. The owner and moderator of one popular consumer discussion forum seemed unaffected by possible intrusions of corporate representatives, and the Head of Operations of a consumer-centric health site suggested such intrusions are discouraged, probably infrequent, and minimally impacting. The Wikiscanner search further indicated that anonymous activity of persons on pharmaceutical company networks in the highly popular Wikipedia was relatively infrequent during 2002-2007.

\section{Data Checking of the Research Sample}

Recent posts vs. older posts.

Tables 4.58 and 4.60, discussed in the previous section, show that the most recent consumer posts for both drugs are comparable to the full dataset on "symptoms improved" and "symptoms new/worsened." Thus, there did not appear to be an imbalance of favorable comments for either drug in the most recent posts.

Table 4.63 divides all consumer posts from 4 websites (excluded IcarusProject) and both drugs by the time period the comment was posted: 2002-2005 and 2006-2009. There were significantly fewer comments in the more recent time period for effects related to sleep, and gastrointestinal and urinary systems. Table 4.64 lists the frequency of consumers who mentioned each code within these 2 categories of effects. Fewer consumers in recent posts mentioned constipation, diarrhea/gas, and nausea/vomiting under gastrointestinal effects, and excessive sleeping/tired, helps me sleep, and trouble 
sleeping/insomnia under sleep effects. However, none of these effects individually reached statistical significance. There also did not appear to be a patterned bias in reporting of sleep effects, as the "positive" effect (helps me sleep) and the remaining 2 "negative" or "adverse" sleep effects were each reported by fewer consumers in the most recent time period.

If a bias of overly favorable reports of drug effects were present, it may be most likely to appear within mental or mood effects. Table 4.64 therefore also compares commonly reported effects within this category against the time period of the post. Significantly more consumers in the recent time period reported vivid dreams or nightmares, and significantly fewer reported an increase in energy, euphoria or mania. Both of these effects could be considered "positive" or "negative" depending on individual context and the severity of the effect. A nearly equal proportion of consumers across time periods mentioned new or worsening symptoms, but significantly fewer consumers in the recent period mentioned an improvement in symptoms $(25.5 \%$ versus $35.0 \%$, respectively). 
Table 4.63

Data Checking by Time Period of Consumers' Posts and Drug Effect Categories for Lexapro and Seroquel Combined

\begin{tabular}{|c|c|c|c|c|}
\hline & $\begin{array}{l}2002-2005 \text { posts } \\
\quad(\mathrm{N}=203)\end{array}$ & $\begin{array}{l}2006-2009 \text { posts } \\
\quad(\mathrm{N}=757)\end{array}$ & $\begin{array}{l}\text { Chi- } \\
\text { square }\end{array}$ & $\begin{array}{c}\mathrm{p}- \\
\text { value }\end{array}$ \\
\hline Sleep Effects & $115(56.7 \%)$ & $348(46.0 \%)$ & 7.312 & 0.007 \\
\hline $\begin{array}{l}\text { Gastrointestinal and Urinary } \\
\text { Effects }\end{array}$ & $30(14.8 \%)$ & $73(9.6 \%)$ & 4.407 & 0.036 \\
\hline $\begin{array}{l}\text { Lab Tests and Chronic } \\
\text { Conditions }\end{array}$ & 2 & 37 & -- & -- \\
\hline Other Effects & 40 & 113 & 2.727 & 0.099 \\
\hline Head or Face Effects & 26 & 68 & 2.652 & 0.103 \\
\hline Appetite and Weight Effects & 47 & 208 & 1.534 & 0.215 \\
\hline Mental or Mood Effects & 122 & 428 & 0.829 & 0.363 \\
\hline Nose Throat Chest Effects & 16 & 73 & 0.591 & 0.442 \\
\hline Skin Effects & 9 & 43 & 0.486 & 0.486 \\
\hline $\begin{array}{l}\text { Musculoskeletal and } \\
\text { Neurological Effects }\end{array}$ & 32 & 114 & 0.062 & 0.804 \\
\hline Sexual Effects & 21 & 81 & 0.021 & 0.884 \\
\hline
\end{tabular}


Table 4.64

Data Checking by Time Period of Consumers' Posts on Selected Code Categories for Both Drugs Combined

\begin{tabular}{|c|c|c|c|c|}
\hline & $\begin{array}{l}2002-2005 \text { posts } \\
\quad(\mathrm{N}=203)\end{array}$ & $\begin{array}{l}2006-2009 \text { posts } \\
\quad(\mathrm{N}=757)\end{array}$ & $\begin{array}{l}\text { Chi- } \\
\text { square }\end{array}$ & $\begin{array}{c}\mathrm{p}- \\
\text { value }\end{array}$ \\
\hline \multicolumn{5}{|l|}{$\begin{array}{l}\text { Gastrointestinal and } \\
\text { Urinary Effects }\end{array}$} \\
\hline Constipation & $2.0 \%$ & $0.8 \%$ & -- & -- \\
\hline Diarrhea / gas & $3.9 \%$ & $1.8 \%$ & 3.127 & 0.077 \\
\hline $\begin{array}{l}\text { Nausea / sick stomach / } \\
\text { vomiting }\end{array}$ & $9.9 \%$ & $6.3 \%$ & 2.999 & 0.083 \\
\hline Urinary changes / problems & $1.0 \%$ & $1.5 \%$ & -- & -- \\
\hline \multicolumn{5}{|l|}{ Sleep Effects } \\
\hline Difficult to wake up & $2.0 \%$ & $3.8 \%$ & -- & -- \\
\hline Extreme sleepiness / tired & $33.5 \%$ & $26.9 \%$ & 3.381 & 0.066 \\
\hline Helps me sleep & $20.7 \%$ & $18.9 \%$ & 0.333 & 0.564 \\
\hline Trouble sleeping / insomnia & $9.4 \%$ & $7.3 \%$ & 0.987 & 0.321 \\
\hline Yawning & $1.5 \%$ & $1.6 \%$ & -- & -- \\
\hline \multicolumn{5}{|l|}{ Mental or Mood Effects } \\
\hline Brain fog / zombie & $13.3 \%$ & $12.8 \%$ & 0.034 & 0.854 \\
\hline Dreams vivid / nightmares & $2.5 \%$ & $6.1 \%$ & 4.155 & 0.042 \\
\hline $\begin{array}{l}\text { Energy increased / euphoria / } \\
\text { mania }\end{array}$ & $6.4 \%$ & $3.3 \%$ & 4.05 & 0.044 \\
\hline $\begin{array}{l}\text { Numb / detached / lack of } \\
\text { energy or interest }\end{array}$ & $7.4 \%$ & $7.7 \%$ & 0.017 & 0.896 \\
\hline Symptoms new / worsened & $12.3 \%$ & $13.3 \%$ & 0.148 & 0.7 \\
\hline Symptoms reduced / improved & $35.0 \%$ & $25.5 \%$ & 7.215 & 0.007 \\
\hline
\end{tabular}




\section{Anonymous versus identifiable posts.}

Table 4.65 shows results of chi-square testing of drug effect categories for consumer reviews posted by anonymous and identifiable users. Two categories, "lab tests and chronic conditions" and "head or face effects," reached statistically significant chi-square values. For the remaining categories, there was no association between reported drug effects and anonymity of the user. Table 4.66 lists the percentage of consumers from each group who mentioned a code within the two statistically significant categories of effects. More consumers who provided an identifiable user name reported problems with increased blood sugar and/or diabetes. More consumers in the anonymous group reported increased headache, hearing/taste/smell changes, and jaw clinching/grinding.

Again, if a bias of favorable accounts were present, it may be more likely to appear within mental or mood effects. There was a non-significant chi-square value for "mental or mood effects" as a category, but selected effects within the category were examined more closely to ensure a thorough review of the data. Table 4.67 shows the number of consumers mentioning the 5 most frequently reported mental or mood effects for Lexapro and Seroquel combined. Only 1 effect, emotional stability or calm, reached statistical significance (chi-square $=4.503, \mathrm{p}=0.034$ ), with more consumers who posted anonymously reporting this effect. Anonymous users also more frequently mentioned new or worsening symptoms ( $\mathrm{p}=0.057)$, and almost equally to identifiable users mentioned improvements in symptoms ( $32.3 \%$ and $27.6 \%$, respectively). Therefore, while there was an imbalance of positive comments relating to emotional stability in the anonymous group, the imbalance did not continue through to other mental or mood effects. 
Table 4.65

Data Checking for Anonymity of Users by Drug Effect Categories for Lexapro and Seroquel Combined

\begin{tabular}{|c|c|c|c|c|}
\hline & $\begin{array}{l}\text { Anonymous Users } \\
\qquad(\mathrm{N}=477)\end{array}$ & $\begin{array}{l}\text { Identifiable Users } \\
\qquad\left(\mathrm{N}=243^{\mathrm{a}}\right)\end{array}$ & $\begin{array}{l}\text { Chi- } \\
\text { square }\end{array}$ & $\begin{array}{c}\mathrm{p}- \\
\text { value }\end{array}$ \\
\hline $\begin{array}{l}\text { Lab Tests and Chronic } \\
\text { Conditions }\end{array}$ & 11 & 13 & 4.629 & 0.031 \\
\hline Head or Face Effects & 57 & 17 & 4.284 & 0.038 \\
\hline Mental or Mood Effects & 307 & 140 & 3.114 & 0.078 \\
\hline Other Effects & 87 & 32 & 3.000 & 0.083 \\
\hline Sleep Effects & 258 & 120 & 1.429 & 0.232 \\
\hline Sexual Effects & 57 & 23 & 1.006 & 0.316 \\
\hline $\begin{array}{l}\text { Gastrointestinal and } \\
\text { Urinary Effects }\end{array}$ & 59 & 26 & 0.431 & 0.512 \\
\hline Nose Throat Chest Effects & 49 & 23 & 0.117 & 0.733 \\
\hline $\begin{array}{l}\text { Appetite and Weight } \\
\text { Effects }\end{array}$ & 134 & 71 & 0.100 & 0.752 \\
\hline Skin Effects & 25 & 13 & 0.004 & 0.951 \\
\hline $\begin{array}{l}\text { Musculoskeletal and } \\
\text { Neurological Effects }\end{array}$ & 76 & 39 & 0.002 & 0.968 \\
\hline
\end{tabular}

${ }^{a}$ Excludes users from CrazyMeds because all users of this forum have an identifiable user name. 
Table 4.66

Data Checking for Anonymity of Consumers on Statistically Significant Code Categories for Both Drugs Combined

\begin{tabular}{|l|c|c|}
\hline \multicolumn{1}{|c|}{$\begin{array}{c}\text { Lab Tests and Chronic } \\
\text { Conditions }\end{array}$} & $\begin{array}{c}\text { Anonymous Users } \\
(\mathrm{N}=477)\end{array}$ & $\begin{array}{c}\text { Identifiable Users } \\
(\mathrm{N}=243)\end{array}$ \\
\hline $\begin{array}{l}\text { Blood pressure changes } \\
\text { Blood sugar high / diabetes }\end{array}$ & $0.6 \%$ & $0.8 \%$ \\
\hline Cholesterol high & $1.0 \%$ & $4.1 \%$ \\
\hline $\begin{array}{l}\text { Liver levels / hepatoxicity / } \\
\text { hepatitis }\end{array}$ & $0.0 \%$ & $0.4 \%$ \\
\hline Other labs or chronic conditions & $0.2 \%$ & $0.8 \%$ \\
\hline \multicolumn{1}{|c|}{ Head or Face Effects } & $0.6 \%$ & $0.8 \%$ \\
\hline Headache / migraines reduced & $1.9 \%$ & $1.6 \%$ \\
\hline Headache increased & $0.4 \%$ & $0.4 \%$ \\
\hline Hearing, taste, smell changes & $5.9 \%$ & $3.7 \%$ \\
\hline Jaw clenching / grinding & $1.3 \%$ & $0.0 \%$ \\
\hline \begin{tabular}{l} 
Vision / eye changes \\
\hline
\end{tabular} & & \\
\hline
\end{tabular}


Table 4.67

Data Checking for Anonymity of Consumers on Selected "Mental or Mood Effects" for Lexapro and Seroquel Combined

\begin{tabular}{|l|c|c|c|c|}
\hline & $\begin{array}{c}\text { Anonymous Users } \\
(\mathrm{N}=477) \\
\mathrm{N}(\%)\end{array}$ & $\begin{array}{c}\text { Identifiable Users } \\
(\mathrm{N}=243) \\
\mathrm{N}(\%)\end{array}$ & $\begin{array}{c}\text { Chi- } \\
\text { square }\end{array}$ & $\begin{array}{c}\mathrm{p}- \\
\text { value }\end{array}$ \\
\hline Symptoms improved & $154(32.3 \%)$ & $67(27.6 \%)$ & 1.681 & 0.195 \\
\hline Emotionally stable / calm & $65(13.6 \%)$ & $20(8.2 \%)$ & 4.503 & 0.034 \\
\hline Symptoms new / worsened & $69(14.5 \%)$ & $23(9.5 \%)$ & 3.612 & 0.057 \\
\hline Brain fog / zombie & $65(13.6 \%)$ & $36(14.8 \%)$ & 0.188 & 0.664 \\
\hline $\begin{array}{l}\text { Numb / detached / lack of } \\
\text { energy or interest }\end{array}$ & $44(9.2 \%)$ & $18(7.4 \%)$ & 0.675 & 0.411 \\
\hline
\end{tabular}

\section{Scripted comments.}

Table 4.68 shows results of the search for 14 common key words and phrases found on the drug manufacturers' websites and medication guides. From the sampling frame of over 7,000 consumer comments, half of the 14 phrases were found in 1 or more comment. For most of the phrases, there was no resemblance between the consumer's comment and information on the manufacturers' websites or medication guides. For 1 phrase, a consumer was citing information from the drug's patient information leaflet. One consumer returned for the phrase "safe" stated that he was a pharmacy technician, but that he took Seroquel and found it to be "SAFE, EFFECTIVE." This consumer advised, "If you follow the drug guidelines and your doctors recommendations you will be fine!" This comment was included in the sampling frame, but was not randomly selected for the coding sample. 
Finally, 1 consumer comment returned for the phrase "takes time" posted an extremely positive comment about Lexapro, "I am so glad that I did (try Lexapro)! It takes time, so stick with it and you will be so glad you did....Lex has rocked my world in the best way. For once in my life I feel like I HAVE a life...For me it has been nothing short of a miracle. I praise and thank God, A LOT!" The search phrases "continue to take" and "takes time" came from the following sentence on Lexapro.com: "Remember, it is important to continue to take your medicine for as long as your healthcare professional advises, even if you start feeling better. Otherwise your symptoms could return or worsen. Full recovery takes time." The above consumer comment, while highly favorable, did not duplicate information on Lexapro.com and was kept in the sampling frame (though it was not randomly selected for the coding sample).

Taking the 3 data checking strategies together, there did not appear to be any consistent patterns of bias. There was an imbalance of favorable comments related to emotional stability by anonymous users, but this imbalance did not appear for other mental/mood effects. Further, there were few differences in reporting according to time period or newness of the post. Since this is a preliminary effort at exploring the authenticity issue of online consumer reviews, continued vigilance and additional probing is certainly necessary. 
Table 4.68

Sampling Frame $(N=7,114)$ Search for "Scripted" Comments

\begin{tabular}{|c|c|c|}
\hline Search terms & $\begin{array}{c}\text { \# of } \\
\text { returns }\end{array}$ & Conclusion about comment(s) \\
\hline Effective in the treatment & 0 & \\
\hline Can be taken every & 0 & \\
\hline $\begin{array}{l}\text { Proven effective; proven to } \\
\text { be an effective }\end{array}$ & 0 & \\
\hline Significantly improved & 2 & No resemblance \\
\hline $\begin{array}{l}\text { Controlled studies; clinical } \\
\text { studies }\end{array}$ & 2 & Cited information from PI sheet \\
\hline $\begin{array}{l}\text { Continue to take your } \\
\text { medicine; continue to take }\end{array}$ & 20 & No resemblance \\
\hline $\begin{array}{l}\text { Full recovery takes time; } \\
\text { takes time }\end{array}$ & 8 & Case \#4367 was a very positive comment \\
\hline Increas* serotonin & 2 & No resemblance \\
\hline $\begin{array}{l}\text { Maintain* treatment; prevent } \\
\text { the depression }\end{array}$ & 1 & No resemblance \\
\hline $\begin{array}{l}\text { Keep taking your medicine; } \\
\text { take your medicine }\end{array}$ & 0 & \\
\hline May take several weeks & 0 & \\
\hline $\begin{array}{l}\text { Work* well and generally } \\
\text { safe; safe }\end{array}$ & 46 & $\begin{array}{l}\text { In case \# 4784, user stated he is a pharmacy } \\
\text { technician, but that he takes Seroquel }\end{array}$ \\
\hline Symptoms don't improve & 0 & \\
\hline $\begin{array}{l}\text { Initial presentation of bipolar; } \\
\text { precipitat* }\end{array}$ & 0 & \\
\hline
\end{tabular}


Chapter 5: Strengths and Limitations

Research Strategy

Coding and analysis for this project relied on the researcher-interpreter as instrument. Researcher bias, including taken-for-granted assumptions, prior knowledge, and personal responses to the data, is a problem for all researchers to handle reflexively and transparently. Several strategies were used in this research to minimize the overall impact of the researcher's perspective. First, coding stuck close to the data, especially for drug effects where little interpretation was needed. Also, theory construction was not part of this research project, which minimized the potential for broad generalizations and unfounded conceptual leaps.

Second, an advantage of using QDA Miner for this project was its ability to track every key stroke. The software included a "Command Log" function in which every command, code, or change to the data was tracked for ultimate transparency. Memos written by the researcher and attached to codes or cases further served to track the evolution of ideas and to make note of unusual cases or uncertain coding decisions.

Finally, a 20\% randomly selected sample of the data was coded by 2 independent coders and acceptable agreement was reached. While it is in the nature of a grounded theory approach for the codebook to continually evolve, for this research many codebook revisions were decided through discussion between the 2 coders (after independent coding was completed).

A primary aim of grounded theory coding is to produce categories that fit closely with the data (Corbin \& Strauss, 2008). The credibility, in terms of the trustworthiness and believability, of the present research was enhanced by minimizing interpretation of 
data, maintaining transparency in the coding process, and including a second independent person to test for agreement.

\section{Authenticity of the Sample}

Due to the inherent anonymity of the Internet coupled with marketing imperatives of pharmaceutical companies, it is possible that pharmaceutical company representatives may be covertly contributing to consumer-constructed sites, thus compromising the integrity of the data as genuine consumer experiences. The credibility of the usergenerated web and the authenticity of individual contributions within specific websites is an unresolved and evolving issue. Further, it is difficult to identify possibly unauthentic accounts, and there is no precedent for a method to accomplish this task. Despite their lack of credibility in certain quarters, however, user-generated sites are highly trafficked and carry utility for their constituents and users.

Revisions to concepts of truth, credibility, legitimacy, and expertise brought on by the Internet are at the heart of discussion and debate among Internet researchers in a variety of fields (David, 2007; Keen, 2007; Warnick, 2007). Adding to this discussion, the present research sought to investigate the similarities and differences in the drug information presented by experts and consumers on various websites. This is to distinguish the aim of the research from one that seeks mainly to determine the truth value of specific claims.

The present research included a few strategies to attempt to check the data for possible bias. All findings were used to nuance the final conclusions regarding the construction of expertise on the Internet. The research compared recent consumer 
comments to earlier comments, anonymous comments to identifiable ones, and searched for "scripted" comments copied from pharmaceutical company material.

Second, the substantial number of contributions on the consumer-constructed sites sampled in this research (minimum 200 contributions) was further intended to reduce the overall impact of possible intrusions. Third, preliminary research was conducted by contacting Web 2.0 researchers, bloggers, and webmasters, and by performing a wikiscanner search of Wikipedia edits.

It is likely that the issue of authenticity on the web will continue unresolved for some time due to the nature of this medium. The information and data resulting from the above strategies are not conclusive evidence, but do offer the suggestion that most consumer accounts can be accepted at face value as genuine consumer accounts. They also suggest the possibility that a small proportion of favorable accounts regarding drugs might be posted by drug company representatives posing as consumers. Given the market imperatives of drug promotion, the immense financial means of the pharmaceutical industry, and the enticing marketing opportunity consumer review sites offer, a skeptical watchfulness regarding this issue is prudent.

The present research sought to contribute to this discussion via the various checks on consumer comments completed as part of data analysis. The selection of websites with a high number of contributors was a final strategy to ensure rich and varied data. None of the above measures, however, can speak to the authenticity of reported consumer experiences as actual experiences, and almost no previous research has sought to investigate this problem. Until systematic assessments of this kind do occur, it cannot be assumed that the data here are genuine consumer experiences; rather they only present 
themselves to be consumer experiences. They also appear to be regarded by most web users as genuine experiences, making an exploration of their content an important, though not wholly sufficient initial step for research. This is the chief limitation of the data, and must be a top priority for continued research if such data is to be made meaningful and useful.

\section{Generalizability and Applicability}

Applicability refers to findings that provide the insight or understanding that allows them to be used in a practical way in policy, practice, or as an addition to a knowledge base (Corbin \& Strauss, 2008). The findings of the present research were not intended to be generalizable in the sense of being representative of a larger population. This research was not able to speak to the unknown population of consumer-constructed or expert-constructed websites on the Internet, but the findings did result in a deeper understanding and insight of the content distributed across the selected sites. The sampling of exemplar sites reflecting typologies further increased the applicability of the finding, which is discussed in more detail in the Conclusion.

\section{Units of Analysis}

Individual consumers were counted as a single case in this study. For the 2 sites with discussion forums, all comments from a user across multiple discussion threads were collected and put together to count as a single case. Some users may have had 1 entry, whereas other users may have posted dozens of comments. It is further likely that the heaviest users - in terms of the number of comments they posted - have a stronger presence and a different standing within the community compared to those who sparsely post. To other community members, the comments of heavy users may hold more weight 
or be more authoritative, and may influence others' comments and the direction of the discussion. The present study did not differentiate between these users, so that consumers who posted 1 time and those who posted 30 times were given the same weight. The analysis, therefore, did not replicate a "typical" reading of this text because it did not effectively capture the nuances and particularities of each online community. To partially address this limitation, measurements of code frequency (which included multiple mentions within a case), rather than case occurrence (which counted each case 1 time regardless of the number of times the code appeared), were sometimes used to reflect the relative attention given to certain themes/effects by heavy users.

\section{Statistical Analysis and Comparisons}

There were a high percentage of consumers who did not report variables of gender, age, and length of time taking the drug. These large amounts of missing data and low cell frequencies prevented some statistical analyses. To work around this problem, some categories were collapsed, such as in the analysis of age group by drug taken where the 2 oldest and 2 youngest age categories were combined.

At other times, sparse reporting of individual drug effects, for example sweating or rash, necessitated examining them as categories of effects, such as "skin effects." The development of categories for individual effects was based on prior knowledge of body systems, as well as the logic and discussion of the two coders. Inter-coder agreement indices, however, were based on categories of effects and acceptable agreement was reached. Regardless, there is inherently some interpretation involved in grouping items together. Whenever statistical tests relied on categories of effects, individual effects within that category were further examined to determine where differences, if any, may 
have resided. Most analyses included some combination of examining effect categories, individual effects, and descriptive excerpts from the data. This strategy of looking at the data in multiple ways was intended as a checks and balance for bias that may have existed at any individual angle.

Another difficulty emerged in finding a common metric to compare expert text and consumer comments. Simple frequencies of the number of cases from each group or how many total times each group mentioned an effect did not provide meaningful comparisons because of differences in the quantities ( $n=4$ for experts, $n=1080$ for consumers) and type (informational text versus consumer reviews) of data. An admittedly cumbersome common metric was found in taking the proportion of mentions of an effect/theme out of all mentions of effects/themes. For example, it was not revealing to state that $100 \%$ of expert cases mentioned the "talking with the doc..." code and just over $16 \%$ of consumers mentioned this code. Similarly, because of the large discrepancy in the amount of data for each group, it was difficult to interpret what it meant for that code to be mentioned 109 times in 4 expert cases and 316 times in 181 consumer cases. However, a comparison could be made in the proportion of those frequencies of mentions out of all mentions, in this case being $52.7 \%$ of all mentions of themes in expert text and $5.1 \%$ in consumer text. While unwieldy and not intuitive to grasp, this metric offered the advantage of estimating the relative attention given by each group to specific effects and themes. This metric was complemented by excerpts of text from each group to add depth and richness to the comparison.

Finally, this was an exploratory study intended for hypothesis generation, rather than hypothesis testing. There was a lot of data to sort through and a large number of 
comparisons were made. In such exploratory studies with many comparisons, it is likely that some significant findings will occur in error. In this study, corrections for multiple comparisons were made in some cases. However, a more general cautious approach to the data was warranted whereby no specific p-value was interpreted too strongly. Rather, conclusions were based on the overall pattern of results. Conclusions of the present research should further serve as planned hypotheses to test in additional future samples. 


\section{Chapter 6: Discussion}

Additional Data on Popularly Reported Drug Effects

\section{Lexapro.}

Consumers taking Lexapro mentioned effects related to sleep, weight, and sex more often than any other type of effects (excluding mental/mood effects). This finding is congruent with other research surveying consumers on "side effects" of antidepressants. iGuard.org, an online service that collects drug safety information from their "patient community," randomly surveyed 700 of its members who were taking at least 1 of 5 antidepressants, including Lexapro (Cascade, Kalali, \& Kennedy 2009). The aim was to gather "real-world" information about antidepressants' side effects. Of the 229 consumers $(38 \%)$ who reported 1 or more side effects, the most frequently reported effects were: problems with sexual functioning (24.5\%), sleepiness (23.1\%), and weight gain (21.4\%). A survey of over 3,000 Consumer Reports' readers being treated for depression and anxiety found a $40 \%$ incidence of sexual dysfunction and $20 \%$ incidence of weight gain (“Drugs vs. talk therapy," 2004). These frequencies are not too different from findings in the present research in which $20.2 \%$ of Lexapro consumers mentioned "sexual effects," 23.8\% excessive sleepiness, and 12.1\% weight gain (although "appetite and weight effects" were mentioned by $22.5 \%$ ).

Controlled trials have reported widely varying rates of sexual dysfunction with antidepressants, sometimes ranging from $20 \%$ to $80 \%$. The $20.2 \%$ rate of mention for sexual effects of Lexapro found in the present study, then, is on the low end of estimates. An 8-month double-blind study comparing Lexapro $(n=274)$ to another antidepressant and placebo found $48.7 \%$ of Lexapro participants reported sexual dysfunction at 8 weeks 
(Clayton, et al., 2007). This was significantly more than that reported on the other antidepressant or placebo (however, the study was funded by the manufacturer of the other antidepressant). The rate fell only slightly by the 8 -month study ending $(43.6 \%)$, but was no longer significantly different from the other drug.

The FDA-approved drug label for Lexapro cites lower rates of sexual dysfunction. Less than $2 \%$ to up to $7 \%$ of clinical trial participants for major depressive disorder and anxiety are listed as experiencing decreased libido or impotence. These effects were described in WebMD as "infrequent" and "unlikely." However, within the category of sexual effects, lost sex drive was the most frequently mentioned effect by consumers in this study (10.6\%). The FDA label further reports that $2 \%$ and $6 \%$ of participants in depression and anxiety trials, respectively, experienced anorgasmia, and $9 \%$ and $14 \%$, respectively, experienced ejaculation disorder. Trouble achieving orgasm was listed as a "common" and "less serious" effect in WebMD, and mentioned by $8.5 \%$ of consumers taking Lexapro. Thus, the officially "infrequent" effect of lost sex drive came up more frequently in the present sample of websites than what is supposed to be the more "common" effect of delayed orgasm.

The drug label for Lexapro also cites lower rates of sleepiness and weight gain compared to consumers in this study and findings from iGuard.org. Somnolence, a term than encompasses drowsiness and sedation, has a $6 \%$ incidence in depression trials and $13 \%$ incidence in anxiety trials according to the drug label, compared to the over $23 \%$ rate of mention in both the present and iGuard.org studies. 
Weight gain is not listed as a side effect on the drug label, rather the following statement is present, "Patients treated with Lexapro in controlled trials did not differ from placebo-treated patients with regard to clinically important change in body weight" (p. 13). Following the lead of the drug label, neither weight gain nor weight loss were mentioned at all on WebMD, and RevolutionHealth only spoke to "weight changes" as "less serious." WebMD did list increased appetite as a "rare" effect. Again $22.5 \%$ of consumers in this study mentioned appetite and weight effects of Lexapro, and over a fifth of consumers in the iGuard.org survey reported weight gain on antidepressants.

The primary "side effects" of Lexapro that were reported by consumers in this study appear congruent with findings from other consumer surveys. However, the rate of sexual effects was on the low end of the varying estimates found in controlled trials. The rates of sleep and weight effects were considerably higher than those listed from controlled trials on the drug label. Descriptions of effects in the 2 expert texts appeared to largely reflect data provided on the FDA-approved drug label.

Of note, the popularity of an effect among consumer reviews may reflect its subjective burden on consumers more than its actual incidence in the drug using population or severity in terms of potential health complications. This limits the usefulness of comparing frequencies across consumer surveys, controlled trials, and the drug label. Caution should be taken not to over-interpret any single finding. Nevertheless, such comparisons are a first step to exploring the similarities and differences of these sources, and some comparisons, such as the above absence of weight gain on the drug label compared to a high rate of mention among consumers, are quite striking. 


\section{Seroquel.}

For Seroquel, the most commonly reported effects by consumers in this study were related to sleep and weight. Over $60 \%$ of consumers mentioned sleep effects, and over $30 \%$ mentioned appetite and weight effects. Antipsychotic induced weight gain is a well-known and concerning issue that can often precipitate more serious metabolic diseases (Correll, et al., 2009; Goudie, Cooper, \& Halford, 2004; Taylor \& McAskill, 2000). On the FDA-approved drug label for Seroquel, there is a confusing discrepancy on the incidence of weight gain in controlled trials. A table on page 17 cites that an average of $22 \%$ of subjects in trials for schizophrenia and bipolar mania, and $8 \%$ in trials for bipolar depression experienced weight gain greater than $7 \%$ of total body weight. However, a table on page 28 that lists all adverse effects in trials for schizophrenia and bipolar mania cites a $5 \%$ incidence of weight gain, and $4 \%$ incidence in trials for bipolar depression. WebMD cited weight gain on Seroquel as "common" and both expert texts described it as less serious or less severe. Over $22 \%$ of consumers in this study mentioned weight gain specifically, corresponding to the highest estimate provided on the drug label. Consumer descriptions of weight gain were not generally congruent with the "less serious" label, as many reported feeling extremely burdened by the extra weight and uncontrollable, ravenous appetite, and occasionally reported a dangerous amount of rapid weight gain.

Off-label use of prescription medications is common, and can exceed $50 \%$ for many psychiatric medications (Radley, Finkelstein, \& Stafford 2006). In the present study, over one-third of consumers taking Seroquel reported that the drug helped them sleep, and nearly the same number reported the drug caused excessive sleepiness. Expert texts included no mention of the drug as a sleep-enhancing agent, as it is not a FDA-approved indication, 
though this appeared to be a popular use of the drug according to consumer accounts. Studies of antipsychotic use in large psychiatric hospitals support this finding and suggest that Seroquel is frequently used in practice to induce sleep (Philip, et al., 2008; Stowell, et al., 2009). An analysis of Veterans Administration data for 2007 showed that over $60 \%$ of the 279,778 unique patients who received an antipsychotic prescription were using the drug for an off-label indication (Leslie, Mohamed, \& Rosenheck, 2009). Seroquel had the largest proportion of off-label use (42.9\%) compared to all other prescribed antipsychotics. Off-label uses included PTSD and Anxiety, among other diagnoses, but did not include insomnia, as data for the study was based on official diagnoses used for prescription reimbursement (use of Seroquel for trouble sleeping is unlikely to show up on those records).

The drug label for Seroquel states, "Somnolence was a commonly reported adverse event" occurring in $18 \%$ of patients in schizophrenia trials, $16 \%$ in bipolar mania trials, and $57 \%$ in bipolar depression trials. Expert text in this study cited "drowsiness" as a "common" and less serious or less severe effect. In a departure from the drug label, expert text further listed insomnia as another "common" effect of Seroquel. The latter effect was mentioned by just over $2 \%$ of consumers in this study, indicating that, at least for this sample of consumers, sleepiness (whether helpful or not helpful) was a tremendously more "common" drug effect than insomnia.

Reports from consumers in this study seem to reflect real-world use of Seroquel as a sleep-aid, among other frequent off-label uses. Appearing to generally reflect data from the drug label, information about the drug's sleep effects on expert text was limited to listing "drowsiness" and "insomnia" as "common" side effects. As an aside, the latter 
effect was barely represented in the present sample of consumers. The other commonly reported effect of Seroquel, weight gain, was listed on the drug label with generally lower incidence rates than the rate of mentions found in the current sample of consumers.

\section{Effect Classification}

The findings from this study support Moncrieff and Cohen's (2006) suggestion that the labels "side effects" and "therapeutic effects" may be misnomers that cannot be easily applied to psychotropic drug effects. One of the more difficult aspects to developing the codebook was in classifying drug effects. The initial codebook included categories for "side effects," "beneficial effects," and effects appearing "neutral." This became impossible to maintain during coding because many of the most commonly reported effects were described by consumers as beneficial, or adverse, or both at the same time. For example, the sleep effect of Seroquel was often described to be a benefit of the drug in the evenings when sleep was needed, and an undesirable effect during the mornings when alertness was needed. In listing drug effects, a consumer on AskaPatient recorded the dilemma as such, "fatigue (good for sleep though)." Memos written by the researcher in December 2009 and February 2010, noted ideas to merge the "helps me sleep" code with the "excessive sleeping" code because they so frequently appeared in the data to be one-in-the-same effect of sedation (i.e., some consumers found the drug to induce just enough sleep, while others found it to induce too much sleep). In the end, the two codes were not merged in the hopes of facilitating a more meaningful analysis of sleep effects.

Also reported, though less often, were the benefits of Lexapro's "side effects" of delayed orgasm and lost sex drive. A minority of consumers were pleased to find they 
"can last longer" during sexual intercourse, and/or that their previously high sex drive was now more "normal."

Similar to the sleep effect described above, some mental or mood effects appeared to manifest on a spectrum. The codes "emotionally stable/calm/normal" and "numb/detached/lack of energy or interest," for example, shared the basic property of a "mellowing" effect that manifested more or less strongly in different individuals. Over $9 \%$ of consumers mentioned that the "calming effect" of Lexapro or Seroquel put them on an "even emotional keel" or "levels out emotions" and allowed them to "handle things better." Just over $7 \%$ of consumers commented that the drug "made me not care about anything," "desensitizes me," made them "numb to the world," "apathetic," and "felt no emotion (happy or sad!).” The mellowing effect was often clearly distinguished as desirable or undesirable by the consumer, though some consumers felt ambivalent about how to classify it. A consumer reviewing Lexapro on RevolutionHealth remarked that the drug produced "such an even keel that it feels strange...I feel like Spock on the old Star Trek series - very rational and systematic, not at all emotional." A user on CrazyMeds commenting on the mellowing effect of Lexapro neatly summarized the problem, "I rather wonder how a person being treated for anxiety is supposed to decide when 'not being concerned' is an undesirable side-effect rather than a desired outcome."

For the purposes of codebook development, it appeared to be more precise to conceptualize that the drugs produced general effects, such as sedation, that some people in certain situations found helpful and some people in certain situations found unhelpful (Moncrieff \& Cohen, 2006). To produce a more accurate representation of consumers' 
accounts, the initial codebook categories (i.e., beneficial, side, and neutral effects) were dissolved early in the coding process in favor of coding all effects simply as "effects."

\section{Variation among Consumers}

Consumer reviews on WebMD and RevolutionHealth were different from those on consumer-constructed sites in important ways. Significantly more consumers on consumer-constructed websites reported Seroquel worsened their symptoms ( $\mathrm{p}=.000)$, while more consumers posting reviews on the expert health sites reported it improved their symptoms $(\mathrm{p}=.008)$. This trend was repeated for Lexapro, but the difference did not reach statistical significance at the $p<.002$ level. In addition to greater reporting of symptom improvement, consumers on expert-constructed sites reported generally lower rates of "side effects." For example, consumers for both drugs on RevolutionHealth reported the highest rate of symptom improvement compared to the other websites. For Lexapro, they reported the lowest rate of weight gain, and for Seroquel, the lowest rate of new or worsening symptoms.

Consumers on CrazyMeds mentioned drug effects at a generally lower rate than consumers on the other 3 websites, but contributed considerably more than the other sites to drug themes. This means that between the 2 consumer-constructed sites reporting effects for specific drugs, consumers on AskaPatient mentioned drug effects at a much higher rate than those on CrazyMeds. AskaPatient also generally reported "side effects" at higher rates than any of the other 4 websites.

These differences may be explained by actual differences in the types of consumers who use these various sites. Many users on CrazyMeds demonstrate an advanced knowledge of psychopharmacology and spend more time discussing drug combinations, 
dosing issues, and the like as opposed to straightforwardly reporting drug effects. It could be reasoned that consumers using AskaPatient may be disconcerted with more mainstream health sources due to negative drug experiences, and looking for an alternative space to find and share such experiences. The structure of AskaPatient supports this hypothesis, as the site specifically solicits information under 2 main fields: "comments" and "side effects." As a logical extension, consumers on WebMD and RevolutionHealth may represent the "average," mainstream drug consumer with an "average" drug experience (i.e., some positive, some negative, but mostly hovering around a middle point). However, the consistent pattern of imbalance that both favored symptom improvement and demonstrated lower rates of side effects on WebMD and RevolutionHealth suggests something other than solely contributions from "average" consumers. There could be several possible explanations. One, rather than "average" consumers, reviews on these sites come from persons with especially positive drug experiences. Two, perhaps expert health sites have filtered consumer drug reviews to reduce overly negative accounts. Such a practice could reasonably be attributed to financial imperatives related to banner advertisements by pharmaceutical companies. Three, the "culture" and virtual physical environment of the websites have influenced the type of comments that are posted. Visual cues, such as medical advertisements and related graphics, may contribute to a "pro-drug" or "pro-medicine" environment that attracts those who share a favorable attitude toward drug use and repel those who have a negative attitude or are more critical of drug use. Over time, normative themes may become established by way of these graphics and the accumulated text contributed by consumers. Users of that site may tend to conform to those normative themes rather than 
post commentary that departs from them. Thus, the structure of the virtual physical and social environment of websites may influence certain behavior patterns of site users.

Regardless, unless the online health searcher who uses consumer reviews actively seeks a variety of sources to retrieve those reviews, these differences in consumers' reporting of drug effects across websites could potentially and unknowingly hinder informed decision-making.

It was hoped as part of this research to also examine differences in reported drug effects according to consumers' gender, age, and length of time taking the drug. If variation had been found, it would add detail and precision that might increase the value of the information as a body of knowledge. Such detail could prove useful for postmarketing surveillance. At the same time, added variation potentially increases ambiguity for individuals making treatment decisions. Most consumers in this sample of online reviews did not provide information on the above variables, which prevented a fruitful analysis of possible differences in reporting. Future research may find it useful to contact consumer reviewers who leave an email address, or use an online survey to gather data that would include these variables.

How do Expert and Consumers Sources Help and Hinder Decision-Making? Drug effects.

Consumer and expert text reported many of the same drug effects, and differed primarily in their descriptions and the attention given to certain effects. First, while expert text listed drug effects, consumer text described drug experiences. Language used to list effects in expert text tended to be vague (i.e., "drowsiness") and fixed (i.e., "less severe"). The information on expert sites (e.g., the professionally-delivered drug 
information on expert sites, which does not include the consumer reviews on those sites) was homogenized and the diversity of experiences was erased. Expert text successfully eliminated ambiguity, making room for more simple and straightforward treatment decisions for health seekers. Expert text appeared to follow the lead of the FDA-approved drug label in providing the official account of drugs' effects. Controlled trials, on which the drug label and presumably expert text is based, are designed to capture averages and report standardized data. It is therefore of little surprise that the language and design of expert text reflected that of its information source.

The information reported by consumers represented a greater diversity of perspectives, and effects were presented in a more specific and relatable manner in terms of providing situational examples. For example, what can be learned from consumers is that "drowsiness" caused by Seroquel can sometimes translate to "coma-like sleep" or having to miss work or school because of the inability to stay awake. For the code "symptoms new/worsened," expert text summarized in list form the adverse mental or mood effects reported in FDA black box warnings on the drug labels. These were the most frequently mentioned effects in expert text. Consumers reported these same effects, but with many examples of how they might manifest in various combinations and to varying degrees (see Table 4.22).

Next to mental or mood effects, expert text most often mentioned physical "side effects" of drugs, such as nausea/vomiting, dizziness, and blood related changes. WebMD (though not RevolutionHealth) listed a number of (usually rare) physical effects that could only be diagnosed with laboratory tests, and that could cause serious disease conditions if left undetected. Only a couple of these effects (i.e., increased blood sugar) 
were discussed by more than a few consumers. Expertise regarding the human body and drugs' actions on the body may be necessary to ensure patient safety in cases of rare adverse effects or those with less obvious manifestation (i.e. that require laboratory tests to detect). While consumer accounts offer rich descriptions of many drug effects, less discussed effects carry the risk of becoming lost in the volume of data.

It is difficult in expert text to decipher the relative burden of lists of effects simply labeled "less serious" and "serious." In this study, those labels applied by experts' did not always match up with the perceived importance or severity of effects according to consumers. One hypothesis is that expert text may label effects according to their impact on the physical body, so that weight gain and sexual dysfunction are generally "less severe" effects while a blood clot in the lung is "severe." Consumers may be more likely to label effects according to the impact on their lives as a whole, including the physical body, psychological and emotional well-being, and social lives. Weight gain becomes a more serious effect judged by its impact on the person's life, while a possible symptom of something more detrimental to the body, such as coughing or trouble breathing, may go undetected because it carries less subjective burden on the life of the person experiencing it. It is arguable, then, whether consistency in expert and consumer descriptions of the "severity" of effects is possible or even desirable. Of course, for many effects, it seems reasonable to assess the effect according to its total impact on a life. Expert text might increase its relevance to consumers by either better explaining the meaning of its current labels or expanding its label system to include effects' broader impact.

A cursory browse (as may be typical for the average Internet user) through consumer reviews offers a strong general impression of what the drug may be "like" through 
concrete, descriptive examples of individuals' experiences. In such a brief review, though, the Internet user would likely miss many less popularly discussed but still potentially momentous effects. There is an increased likelihood when relying on consumer accounts that persons might miss important information about potential drug effects that can only be detected on the basis of laboratory tests. In contrast, for the "typical" online health seeker, expert text provides succinct and readable summaries of both common and rare, less serious and serious drug effects.

Finally, experts and consumers differed in the attention each group gave certain effects. Consumers taking both drugs appeared to be most concerned with effects on symptoms, sleep, and weight, whereas expert text primarily attended to physical effects. Consumers taking Lexapro frequently discussed weight gain, though this effect did not appear at all in 1 expert text and only appeared as "weight changes" in the other. About one-third of consumers taking Seroquel reported the drug helped them sleep, but there was no mention in expert text of the drug's potential beneficial or desirable impact on sleep. Expert text simply listed "drowsiness."

In sum, expert and consumer text reported many of the same drug effects, but the relative attention given to particular effects was not distributed similarly among the groups and descriptions of drug effects were often qualitatively different. Consumers frequently discussed effects that were either more obvious or especially burdensome, such as weight gain, changes in sleep, and mental or mood changes. Other than repetitions of the black box warning from the drugs' labels, expert text more frequently mentioned physical effects, which are more likely to be manifested in less obvious ways or carry fewer practical burdens. Consumers offered full and descriptive accounts of drug 
effects, but the usability of this information is limited by the volume of data that must be sorted through. Expert text offered succinct and comprehensive summaries of drug effects, but the meaningfulness of this information is limited by the lack of context. In answer to the first research question, "Does the accumulated consumer knowledge that the Internet allows dissolve the traditional boundary between expert/scientific and consumer/lay knowledge?" it seems that both groups have legitimate claims to expertise, and both forms of knowledge (that based on the scientific method and that based on experience) are beneficial for informed decision-making. The limitations of one are made up by the advantages of the other. However, if consumer reports were more organized and accessible (their major limitation currently), then the expert accounts found on WebMD and RevolutionHealth could arguably be considered superfluous. Similarly, if expert accounts increased their relevance by more richly describing the range of drug effects, then online consumer reviews may not be such a necessary innovation for the thousands of active and potential drug consumers who rely on them.

\section{Themes.}

Consumers mentioned more than double the number of themes found in expert text in relation to drugs, doctors, family and friends, expense and insurance, pharmaceutical companies, and general support and advice. Among the themes overlapping between experts and consumers, consumers showed a range and diversity of perspectives, while expert text reflected a more uniform discourse. For example, expert text provided lengthy lists of possible drug "side effects," but only 1 statement on 1 of the sites referenced an assessment of benefits and costs, and it was clearly in favor of drug use. As part of their drug reviews, consumers often made assessments of benefits and costs, and came to 
favorable, unfavorable, and uncertain decisions. More often than anything, consumers came to the conclusion that the drug was for any variety of reasons of limited benefited. Consumers also stressed that each person must make their own individual assessment ("your mileage may vary"), which is important given the lack of predictability of psychotropic drug effects.

There was a strikingly uniform discourse in expert text around "the doctor." Expert text was adamant, as measured by the number of times the code appeared in the text as well as the percentage of words from the total text that received the code, about telling, calling, talking to, consulting with, discussing with, or otherwise making no drug-related decision without getting instruction from the doctor. It was the most frequently mentioned of all themes in expert text, and the only theme involving the doctor that was repeated continuously throughout each section of expert text. This circular pattern of selfreferences whereby whatever the problem, the doctor holds the answer serves to strengthen the popularly accepted notion of an esteemed status and specialized knowledge of the medical doctor.

Consumers appeared to rely on their general practitioner or psychiatrist for their skills and technical expertise in medicine, and, with some exceptions, the research found little evidence that consumers were trying to exclude their doctor from their care and treatment by searching and sharing information online. This conclusion is supported by recent focus group research finding that online health seekers felt empowered by instant access to information, but were still unwilling to give up face-to-face interactions with their doctor (Donnelly, Shaw, \& van den Akker 2008). Comments about telling, talking to, discussing with, et cetera the doctor were frequent in consumer text. The difference 
between expert and consumer text was that consumers also demonstrated a range of other interactions with and feelings towards the doctor. As discussed in the review of literature, previous research has demonstrated insufficiencies in the communicating of drug information from doctors to patients/consumers. Those findings are further supported by consumer comments in this study.

Consumer reviews offered the advantage of a diversity of perspectives, as there is no single consumer "voice" parallel to the unitary biomedical discourse found on mainstream professional health sites. In parallel, expert text seemed to reinforce the conventional stereotype of the omniscient doctor and the construction of "medication" as an expert domain.

\section{Theoretical Explanations of Findings}

This research used Foucault's ideas of power/knowledge and a politics of truth as the conceptual framework for analysis. Foucault's description of knowledge as dispersed, often in an incomplete and contradictory manner, appears to be supported by the findings of this research. Each consumer (and expert) offered a piece of a larger puzzle by highlighting different aspects of the varieties of drug experiences. There was no uniform discourse among consumers, but instead multiple, sometimes overlapping and sometimes contradictory discourses were present. The extent to which the effects reported by consumers were already informed by "expert" information is unknown, and should temper the conclusions. However, it appeared that given the opportunity to contribute directly to a knowledge field, particularly one involving an "experience good" like psychotropic drugs, consumers were able to identify and describe many of the same effects reported as part of more scientific methods. If such knowledge is dispersed, as this 
research suggests, then the question becomes one of understanding how the power associated with knowledge becomes linked to one set of truth-making procedures and not another. That is, if experts and consumers can both legitimately and meaningfully contribute to a body of knowledge utilizing their respective resources (scientific method and experience), then differences in status, attention, and influence of that knowledge source may relate to power.

While Foucauldian concepts framed the original research, other theoretical explanations of the findings are possible. A more pragmatic framework might alternatively argue that the domain of medicine as an expertise is the human body, and that the expert text in this research provided a comprehensive and concise accounting of drugs' effects in this regard. Its labels rightly represented the impact of an effect on the body. Expert text also provided information about important effects that would likely go unnoticed by those without medical expertise. Thus, medical expertise in the domain of psychotropic drugs may be essential and beneficial to those who need it. Further, while consumers reported many of the same effects listed in expert text, the presence of bias and lack of structure inherent in this data source has limited its usefulness (and therefore its use) in the construction of drug knowledge. The scientific method (in its ideal form), with its systematic attempts to decipher true effects from noise, may be preferable to an unsystematic and (in this case) largely anonymous collection of presumably consumer voices. Still, consumer accounts do appear to add valuable contextual information to the understanding of drugs' effects and their impact on a person's life. The expert sites in this study may have recognized this fact, as evidenced by opportunities provided for consumers on the sites to rate and write about their drug experiences. 
The findings of this study could also be interpreted in terms of evolving tensions between expertise and democracy, with the Internet representing an expansion of Habermas' public sphere. Never before has there been the opportunity for public participation in constructing official drug knowledge or for potentially identifying drug problems. Online patient communities, such as PatientsLikeMe and iGuard, are capitalizing on this expansion of consumers' roles. Consumer comments analyzed in the present study did not support fears of some analysts who claim amateur or lay content is uninformed, possibly dangerous, and pushes out necessary expertise (Keen, 2007; Siegel, 2008). On the contrary, consumers reported many of the same effects as listed on expert sites, though with sometimes major differences in the evaluation of effects' significance. These differences could not be used to label consumers' reports as less accurate or of lesser quality, but rather may best serve as a supplement to the typically briefer and more narrowly focused expert descriptions. Further, some critical theorists argue that the Internet is democratizing only inasmuch as marginalized or silenced voices are represented (Dahlberg \& Siapera, 2007). Expert sites in this study provided opportunities for consumers (a traditionally silenced group in clinical drug research) to have "voice," thereby supporting the notion of the Internet as a democratizing space. However, important differences were found between consumer accounts on expert-run and consumer-run sites, along with considerable imbalances in the number of consumers expressing certain themes or topics of concern between sites. As mentioned, these differences may reflect consumers' attempts to maintain congruence with the norms of the community (website). They may also suggest that there exist real sub-communities of psychotropic drug consumers who have shared experiences and interests related to drug 
use. Habermas' public sphere was modified by Hauser who presented the notion of a "rhetorical public sphere," which is "a discursive space in which strangers discuss issues they perceive to be of consequence to them and their group" $(1999$, p. 64). The activity of members in a rhetorical public sphere is issue-oriented, then, rather than class-based, which may help conceptualize the distinct interests and concerns of consumers across websites in this study. The Internet may facilitate the expansion of rhetorical public spheres, with the sample of websites in the present study perhaps reflecting this evolution in its infancy. As online patient communities continue to grow and additional research sheds light on their advantages and disadvantages, it is possible that this new form of a public sphere might influence regulatory policy for drugs, including safety surveillance, the classification of unpleasant effects as "serious" or other, and the conduct of research. 


\section{Chapter 7: Conclusion}

\section{Hypotheses for Future Research}

The grounded theory approach used in this study was intended as an exploratory step that would assist in the formulation of hypotheses for more structured future research. Several questions emerged. First, the "real" frequencies of sleep, weight, and sexual effects of Lexapro need to be further investigated. Considerable discrepancies exist between frequencies of mention of these effects in this study, which are supported by other consumer surveys, and incidence rates in the published drug label. Does the high percentage of consumer reports of these effects reflect an actual greater incidence than that captured (or reported) in trials analyzed by the FDA? If these effects are in fact occurring at a higher incidence, then how was this missed in large-sized clinical drug trials? Alternatively, does the higher percentage found in consumer reports only reflect the perceived burden or some other aspect of these effects, but not an actual higher frequency of occurrence? A couple of research strategies could examine these questions. A large, known (i.e., face-to-face) population of Lexapro consumers could be followed for cross-sectional or longitudinal data of drug effects based on structured checklists and open-ended response. Controlled trials could incorporate simple targeted measures for weight, sleep and sexual effects, rather than continue to rely on "spontaneous" or "unsolicited" self-report for such data (a method which tends to underestimate the true frequency of events) (Greenhill, et al., 2003; Hughes \& Cohen, 2010).

For more serious drug effects, data from this study or additional studies of consumer reports could be compared to data from the main mechanism through which professionals report serious effects they observe in patients, FDA's MedWatch. The MedWatch system 
relies on voluntary reports primarily from medical professionals to detect safety issues once a drug has been released to the market. A comparison of consumer and professional voluntary reports might shed some light on the use of a consumer platform for safety surveillance. A number of details should be included in an adverse drug reaction report in order for the report to be meaningful, including age, gender, weight, length of time on the drug, intensity or severity of the effect, drug history, family history, effect of withdrawal, and effect of rechallenge, among other items. Future research could examine how well consumers already report these items in online consumer reviews, and make further suggestions for how to improve consumer reporting of drug effects. These findings can also be compared to the average completeness of MedWatch reports, which are specifically designed to elicit this information. Second, according to findings from this study and published research from large hospitals, Seroquel's primary use and effect is related to sleep. This is an off-label use of the drug, and not an "officially" recognized primary drug effect (as "drowsiness" is only listed as a "less serious" "side effect"). Future research can examine more closely differences between diagnosis and reason for use. One hypothesis is that while the official diagnosis or indication (listed for insurance reimbursement) may be, for example, bipolar mania, the "reason" for prescribing or using Seroquel specifically may be more narrowly related to its desirable effects on sleep. This hypothesis runs parallel to Moncrieff and Cohen's (2009) argument that psychotropic drugs have global effects, rather than specific effects on presumed neurobiological abnormalities. Consumer review data such as that used in this study could be examined for congruence between diagnosis, which was often listed or stated by consumers, and reason for use, which was often explained as part of the total review. 
The present study did not explore differences in drug effects grouped by diagnosis or reason for use. By breaking down frequencies of some "side effects" of Seroquel by indication (bipolar mania, schizophrenia, bipolar depression), the drug label implies that drugs have specific neurobiological action and that drug effects will differ according to the condition being treated. The label sometimes lists considerable differences in frequencies of effects among indications, such as a much higher rate of somnolence in bipolar depression trials. Of course, it is unknown whether methodological issues may have contributed to inflated or deflated estimates in some studies. While not exploring diagnosis, the present study did find that drug effects were not easily parceled into the categories listed on expert sources and the drug label (i.e., "side effects," "less serious," etc.). As an alternative explanation to neurobiological specificity, perhaps the global effect of sleep is experienced and reported differently by a person presenting with, for example, a depressed versus an excitable mood. This hypothesis is supported by classic studies of drug use in which 3 factors were found to influence drugs' effects: 1) drug (the pharmacologic action of the drug itself), 2) set (the person's state of mind, mood, and attitude at the time of drug use), and 3) setting (the physical and social setting within which the drug use occurs) (Zinberg, 1986). Also, as mentioned in the Literature Review, psychotropic drugs' effects are unpredictable for any 1 person, and may even differ within any 1 individual taking the drug at different points in times (Brunton, Lazo, \& Parker, 2006). To investigate neurobiological specificity versus global drug effects, additional studies examining drug effects in healthy, normal volunteers could help determine whether primary drug effects, as Moncrieff and Cohen suggest, occur regardless of psychiatric diagnosis. Researchers conducting controlled trials of drugs 
could broaden their scope of inquiry to include a greater range of psychological and behavioral effects (rather than a narrow focus on physical effects), as well as variables that might influence the drug experience, such as patient expectation, positive or negative suggestion by the doctor or researcher, and patients' personality characteristics.

Future Research: Authenticity and Credibility

A troubling limitation of the online medium in general is the inherently unknown authenticity of persons contributing to content. It is imperative that the investigation of authenticity precede the continued development of and reliance on online consumers as a legitimate information source. Authenticity is one of a few issues at the center of the debate about filtering online searches to retrieve only "trusted" health information sources, and is principally directed towards consumer-generated sites (as well as "gimmick" advertising sites, such as online pharmacies). As expert health sites are tacitly "trustworthy," perhaps due to the alignment of their discourse with science and medicine, the burden of proof for trustworthiness more often falls on non-expert sources. Future research should investigate the authenticity of online consumer drug accounts in a way that does not implicitly favor scientific ways of knowing while forcing those offering experiential knowledge to prove their worth.

Currently, the authenticity problem is unresolved and only a few scrutinizing attempts have been made in any field of study. For consumer drug reviews, the findings from this study could be replicated using additional samples, websites, and drugs, and compared to other online consumer samples, expert health information, and drug literature. 
Another important next step is to compare online consumer comments (with unknown authenticity) about specific drugs to face-to-face samples of consumers (with known authenticity). If feedback from real-world community samples is found to be equivalent to online samples, then this may provide a foundation for the continued development of online technology to support consumer-generated drug information (see next section). Important differences in face-to-face and online samples may provide some interesting and useful insights into who really is contributing to "consumer"-generated online content.

\section{Future Research: Usability}

Consumer drug accounts are scattered across the Internet, making the "usability" of this information a chief limitation and a top priority for continued research and development. First, as there is no "typical" online health search that can be replicated, several samples drawn in different ways should be analyzed for representativeness as was done in this study with the most recent 20 posts from each website. While AskaPatient lists consumer reviews by date, WebMD sorts reviews according to diagnosis then date, and RevolutionHealth by diagnosis then "most helpful." It is likely that a "typical" search would include exposure to any number of combinations of reviews based on the particular navigation pathways and interests of the user. The preliminary work of the present study should be expanded to replicate additional possible searches.

The present study explored the content of online consumer reports, but a more structured examination of specific variables (such as items needed for an adverse drug reaction report, listed in the previous section) is necessary to further devise strategies that might increase the usability of this information source. One hypothesis stemming from 
the present research is that many consumers naturally report at least some important items within the context of describing their drug experience. This may happen sporadically, though, as evidenced by the large amount of missing data in this study for gender, age, and length of time on drug. However, the 2 websites that specifically elicited that information (WedMD and AskaPatient) had very high rates of completed data. It appears then that consumers may be willing to provide additional details if asked for them. Existing and developing websites that collect consumer accounts could enhance their relevance to safety surveillance and opportunities for more advanced decisionmaking by eliciting such information.

To further increase usability, existing and developing websites could also integrate an advanced search function that would allow users to search consumer comments for key words, such as "weight" or "tingling," as well as return thesaurus terms associated with those key words, such as "appetite" with "weight" or "zaps" with "tingling." Currently, the websites reviewed in this study sort consumer reviews according to date posted, indication, or helpfulness, with no additional options for organizing or filtering the hundreds or thousands of comments.

An additional step would be to develop a "meta" website that integrates the mass of dispersed online consumer reviews, and somehow organizes them in a logical and useful way. Currently there are patient communities, such as patientslikeme.com and iGaurd.org, which attempt to not only collect consumer accounts (like AskaPatient), but also to organize and report back in summary form the data from its users. These sites rely on the dedication of their users to monitor and report changes in their condition and treatment on an ongoing basis. Thus, it may be reasonable to assume that these sites 
represent a more pro-active and possibly informed group of healthcare consumers, compared to those who do not participate in consumer-generated online media and even to those whose participation is limited to writing a single drug review on WebMD, for example. The present research found important differences among drug consumers who posted a review or comment on various websites. To achieve the most balanced and representative snapshot of consumer experiences, then, these differences need to be accounted for. Individual online patient communities are innovative and useful for many reasons, but they are limited in the preceding respect.

However, there are currently websites for travel (kayak.com) and consumer home and electronic goods (consumersearch.com) that pull together information from multiple sites and present it in a single organized site. Kayak.com searches travel deals from all the major travel sites, such as cheaptickets, travelocity, expedia, and so on, to bring the user the best deals without having to visit each site individually. Consumersearch.com brings together expert reviews on consumer goods, such as reviews from PC Magazine and PC World on a laptop, and lists each full review as well as provides a summary of pros and cons based on an assessment of all the reviews. It appears that the technology exists to develop the usability of consumer drug reviews, and future research should explore the logistics for its application to this domain. Such a "meta" website would prove useful for individual drug decision-making, as well as hold potential for active post-marketing safety surveillance.

Searching for Health Information on the Internet 
Currently, search engines such as Google are optimizing health searches to return government and other institutional sources at higher rankings, to the exclusion of (consumer delivered) sources lacking "quality" seals of approval (Fox, 2010). Approximately two-thirds of online health searches start at a general search engine, and Google leads the way garnering over $72 \%$ of U.S. searches. Thus, search results can "make or break" a website in terms of access and attention. Previously, all searches in Google returned websites according to "the 'democratic' PageRank algorithm," which favored websites having, for example, a high number of links in and links out, high site traffic, ample metadata or key words, and frequent site updating. This somewhat leveled the playing field for large and small websites, as the usefulness and relevance of the site mattered more than the resources of the site owners.

Google's latest initiative, in response to the ongoing debate over health information quality on the Internet, is "to guide consumers to safe, trusted health websites" (Fox, 2010, para. 3). This has resulted in a new mechanism for ranking websites in health searches, whereby the most widely used expert health sites featuring quality seals of approval, including WebMD, MayoClinic, and Medline, are highlighted at the top of returns (preceded of course by "sponsored links," or advertisements). This search return strategy clearly and openly favors the "big players" with networks of human, knowledge, and capital resources.

However, this research suggests that the "dangerousness" of consumer delivered drug information on the Internet may be exaggerated. A cursory review of consumer drug reviews, reminiscent of the average web user, reflected drug effects in fair proportion to a full representative sample of consumer reviews, which in turn was largely comparable to 
effects listed on expert health sites. In this study, both expert and consumer information sources offered distinct advantages and disadvantages. It does not appear to be true, then, that the "best" information is universally to be found on the biggest, most well-resourced, expert-run health sites.

The questionable objectivity and credibility of expert-derived drug information, as outlined extensively in the review of literature, warrants a parallel, though rarely considered caution when seeking information for making treatment decisions. In fact, it is safe to say that the objectivity and credibility of both expert and consumer sources can be legitimately questioned, and both sources again have benefits and limitations for decision-making. The disparity in labeling sources "trustworthy" and "quality" may reflect the power accorded to procedures of science and scientific/medical discourse more than actual differences in the trustworthiness or other quality measures of information. The promise of the Internet to "democratize" participation in constructing and delivering knowledge in a variety of fields is dying in favor of the status quo of the off-line world. As the historical advocates for the powerless and silenced, social workers are encouraged to promote democratization on the Internet by seeking out consumer delivered health sites at least as frequently as other sources during online searches. Practitioners can also take the additional time necessary to find and utilize the most useful and relevant sites (whatever the information source) rather than simply those that appear at the top of search engine returns. These recommendations are not meant to favor consumer over expert health sources, but to promote that potential of a level playing field that the Internet theoretically allows. Social work practitioners should also support clients who show interest in participating in the consumer-generated online realm of healthcare. 
Until the above investigations concerning authenticity and usability come to pass, clinicians and consumers seeking drug information on the Internet may want to be open, but cautious when reviewing consumer-generated content. A suitable approach may be to read a similar number of reviews from multiple sources, as done in the present research, in order to maximize the possibility of exposure to a variety of drug accounts. Anything less, such as reading 5 or 10 reviews from a single website, should be avoided, especially when the information is being gathered for anything more than simple curiosity. It should also be noted that, at least in the current online environment, consumer-generated information is complementary to, not in replacement of, a thorough review (certainly by social work professionals, if not consumers too) of other drug information sources.

LIST OF REFERENCES 
2007 web awards winners. (2008). Retrieved March 13, 2008, from http://www.strategichealthcare.com/awards/winners.php

Abbott, A. (1988). The system of professions: An essay on the division of expert labor. Chicago: The University of Chicago Press.

Allan, K. (2006). Contemporary social and sociological theory. Thousand Oaks: Pine Forge Press.

Angen, M. (2000). Evaluating interpretive inquiry: Reviewing the validity debate and opening the dialogue. Qualitative Health Research, 10(3), 378-395.

AOL co-founder to launch revolutionhealth.com. (2007). Retrieved June 18, 2008, from http://abcnews.go.com/GMA/OnCall/story?id=2859378\&page=1

Applbaum, K. (2006). Pharmaceutical marketing and the invention of the medical consumer. PLoS Medicine, 3(4), e189.

Association, A. P. (1994). Diagnostic and statistical manual of mental disorders (4th ed.). Washington, D.C.: American Psychiatric Press.

Association, A. P. (1998). DSM-IV sourcebook. Washington, D.C.: American Psychiatric Association.

Avorn, J. (2005). FDA standards - Good enough for government work? N Engl J Med, $353(10), 969-972$.

Avorn, J. (2006). Highlights and a hidden hazard - The FDA's new labeling regulations. New England Journal of Medicine, 354(23), 2409-2411.

Avorn, J. (2007). Paying for drug approvals - Who's using whom? N Engl J Med, 356(17), 1697-1700.

Azoulay, P. (2002). Do pharmaceutical sales respond to scientific evidence? Journal of Economics \& Management Strategy, 11(4), 551-594.

Barker, K. (2008). Electronic support groups, patient-consumers, and medicalization: the case of contested illness. Journal of Health and Social Behavior, 49, 20-36.

Bekelman, J., Mphil, Y., \& Gross, C. (2003). Scope and impact of financial conflicts of interest in biomedical research. JAMA, 289(4), 454-465.

Berg, B. (2000). Qualitative research methods for the social sciences (4th ed.). Boston, MA: Allyn and Bacon.

Berger, P., \& Luckmann, T. (1967). The social construction of reality: A treatise on the sociology of knowledge. Garden City, NY: Anchor. 
Bourdieu, P. (1988). Vive la crise! For heterodoxy in social science. Theory and Society, $17,773-787$.

Bowskill, R., Clatworthy, J., Parham, R., Rank, T., \& Horne, R. (2007). Patients' perceptions of information received about medication prescribed for bipolar disorder: Implications for information choice. Journal of Affective Disorders, 100, 253-257.

Brown, P. (2002). WHO calls for a health domain name to help consumers. British Medical Journal, 324(7337), 566.

Brunton, L., Lazo, J., \& Parker, K. (Eds.). (2006). Goodman \& Gilman's the pharmacological basis of therapeutics (11 ed.). New York: McGraw-Hill.

Busfield, J. (2006). Pills, power, people: Sociological understandings of the pharmaceutical industry. Sociology, 40(2), 297-314.

Carnevale, D. (2006, January 13). Journal gives Wikipedia high marks for accuracy, but not for readability. The Chronical of Higher Education.

Caron-Flinterman, J., Broerse, J., \& Bunders, J. (2005). The experiential knowledge of patients: A new resource for biomedical research? Social Science \& Medicine, 60, 2575-2584.

Cascade, E., Kalali, A., \& Kennedy, S. (2009). Real-world data on SSRI antidepressant side effects. Psychiatry, 6(2), 16-18.

Charlin, B., Tardif, J., \& Boshuizen, H. (2000). Scripts and medical diagnostic knowledge: Theory and applications for clinical reasoning instruction and research. Academic Medicine, 75(2), 182-190.

Charlton, B. (2001). Fundamental deficiencies in the megatrial methodology. Current Controlled Trials in Cardiovascular Medicine, 2(1), 2-7.

Charmaz, K. (2006). Constructing grounded theory: A practical guide through qualitative analysis. Thousand Oaks: SAGE Publications.

Christmas, D. (2005). 'Brain shivers': From chat room to clinic. Psychiatric Bulletin, 29, 219-221.

Clarke, A. (2005). Situational analysis: Grounded theory after the postmodern turn. Thousand Oaks: SAGE Publications.

Clarke, A., Mamo, L., Fishman, J., Shim, J., \& Fosket, J. (2003). Biomedicalization: Technoscientific transformations of health, illness, and U.S. biomedicine. American Sociological Review, 68, 161-194. 
Clayton, A., Kornstein, S., Prakash, A., Mallinckrodt, C., \& Wohlreich, M. (2007). Changes in sexual functioning associated with duloxetine, escitalopram, and placebo in the treatment of patients with major depressive disorder. Journal of Sexual Medicine, 4, 917-929.

Cohen, D. (2003). The psychiatric medication history: Context, purpose, and method. Social Work in Mental Health, 1(4), 5-28.

Cohen, D. (2005). Clinical psychopharmacology trials: "Gold standard" or fool's gold? In S. Kirk (Ed.), Mental disorders in the social environment: Critical perspectives (pp. 347-367). New York: Columbia University Press.

Cohen, D. (2007). Helping individuals withdraw from psychiatric drugs. Journal of College Student Psychotherapy, 21(3/4), 199-224.

Cohen, D., Hughes, S., \& Jacobs, D. (in press). The deficiencies of drug treatment: The case of Strattera. In S. Timimi \& J. Leo (Eds.), Rethinking ADHD: International perspectives. New York: Palgrave Macmillan.

Cohen, D., \& Jacobs, D. (2007). Randomized, controlled trials of antidepressants: Clinically and scientifically irrelevant. Debates in Neuroscience, 1, 44-54.

Cohen, D., McCubbin, M., Collin, J., \& Perodeau, G. (2001). Medications as social phenomena. Health, 5(4), 441-469.

Collins, H., \& Evans, R. (2007). Rethinking expertise. Chicago: University of Chicago Press.

Collins, H., \& Pinch, T. (2005). Dr. Golem: How to think about medicine. Chicago: The University of Chicago Press.

Conrad, P. (2005). The shifting engines of medicalization. Journal of Health and Social Behavior, 46, 3-14.

Conrad, P. (2007). The medicalization of society. Baltimore: The Johns Hopkins University Press.

Conrad, P., \& Leiter, V. (2004). Medicalization, markets and consumers. Journal of Health and Social Behavior, 45, 158-176.

Corbin, J., \& Strauss, A. (2008). Basics of qualitative research (3rd ed.). Los Angeles: SAGE Publications.

Correll, C., Manu, P., Olshanskiy, V., Napolitano, B., Kane, J., \& Malhotra, A. (2009). Cardiometabolic risk of second-generation antipsychotic medications during first- 
time use in children and adolescents. Journal of the American Medical Association, 16, 1765-1773.

Corrigan, O. (2002). A risky business: The detection of adverse drug reactions in clinical trials and post-marketing exercises. Social Science \& Medicine, 55, 497-507.

Dahlberg, L. (2005). The corporate colonization of online attention and the marginalization of critical communication? Journal of Communication Inquiry, 29(2), 160-180.

Dahlberg, L., \& Siapera, E. (Eds.). (2007). Radical democracy and the Internet: Interrogating theory and practice. New York: Palgrave-Macmillan.

David, S. (2007). Toward participatory expertise. In J. Karaganis (Ed.), Structures of participation in digital culture (pp. 177-196). New York: Social Science Research Council.

David, S., \& Pinch, T. (2005). Six degress of reputation: The use and abuse of online review and recommendation systems. Retrieved February 12, 2008, from http:ssrn.com/abstract $=857505$

Davis, J. (2006). Suffering, pharmacuetical advertising, and the face of mental illness. The Hedgehog Review, Fall, 62-77.

Davis, J., Cross, E., \& Crowley, J. (2007). Pharmaceutical websites and the communication of risk information. Journal of Health Communication, 12, 29-39.

Denscombe, M. (2007). The good research guide for small-scale social research projects (3rd ed.). London: Open University Press.

Diving deeper into online health search. (2007). Retrieved May 22, 2008, from http://www.envisionsolutionsnow.com/healthsearch.html

Dixon-Woods, M. (2001). Writing wrongs? An analysis of published discourses about the use of patient information leaflets. Soc Sci Med, 52, 1417-1432.

Donahue, J., Cevasco, M., \& Rosenthal, M. (2007). A decade of direct-to-consumer advertising of prescription drugs. $N$ Engl J Med, 357(7), 673-681.

Donnelly, L., Shaw, R., \& van den Akker, O. (2008). eHealth as a challenge to 'expert' power: A focus group study of Internet use for health information and management. Journal of the Royal Society of Medicine, 101(10), 501-506. 
Drugs vs. talk therapy. (2004). Retrieved April 28, 2010, from http://www.consumerreports.org/health/free-highlights/manage-yourhealth/depression/talktherapy.htm

Dyson, S., \& Brown, B. (2006). Social theory and applied health research. New York: Open University Press.

Elkin, N. (2008). How America searches: Health and wellness. Retrieved July 3, 2008, from http://www.icrossing.com/research/how-america-searches-health-andwellness.php

Eng, T., \& Beauchamp, N. (2005). The role of the Internet in supporting consumer health decision making. In R. Rich \& C. Erbs (Eds.), Consumer choice: Social welfare and health policy. New Brunswick: Transaction Publishers.

Eysenbach, G. (2008). Credibility of health information and digital media. In M. Metzger \& A. Flanagin (Eds.), Digital media, youth, and credibility (pp. 123-154). Cambridge, MA: The MIT Press.

Eysenbach, G., Powell, J., Kuss, O., \& Sa, E. (2002). Empirical studies assessing the quality of health information for consumers on the world wide web: A systematic review. JAMA, 287(20), 2691-2700.

Eysenbach, G., \& Till, J. (2001). Ethical issues in qualitative research on internet communities. British Medical Journal, 323, 1103-1105.

Ferguson, T. (2002). From patients to end users: Quality of online patient networks needs more attention than quality of online health information. British Medical Journal, 324(7337), 555.

Foucault, M. (1980). Power/Knowledge: Selected interviews and other writings 19721977 (C. Gordon, L. Marshall, J. Mepham \& K. Soper, Trans.). New York: Pantheon Books.

Fox, S. (2010). Health sites: Some are more equal than others. Retrieved April 27, 2010, from http://www.pewinternet.org/Commentary/2010/January/Health-Sites-SomeAre-More-Equal-Than-Others.aspx\#

Fox, N., \& Ward, K. (2006). Health identities: From expert patient to resisting consumer. Health, 10(4), 461-479.

Freund, P., \& McGuire, M. (1999). Health, illness, and the social body (3rd ed.). Upper Saddle River, NJ: Prentice-Hall. 
Gabe, J., \& Calnan, M. (2000). Health care and consumption. In S. Williams, J. Gabe \& M. Calnan (Eds.), Health, medicine and society: Key theories, future agendas (pp. 255-273). New York: Routledge.

Gabe, J., Kelleher, D., \& Williams, G. (1994). Challenging Medicine. New York: Routledge.

Gagnon, M., \& Lexchin, J. (2008). The cost of pushing pills: A new estimate of pharmaceutical promotion expenditures in the United States. PLoS Medicine, 5(1), 29-33.

Gardner, P. (2003). Distorted packaging: Marketing depression as illness, drugs as cure. Journal of Medical Humanities, 24(1/2), 105-130.

Garfield, S., Francis, S., \& Smith, F. (2004). Building concordant relationships with patients starting antidepressant medication. Patient Education and Counseling, $55,241-246$.

Gilbert, D., Walley, T., \& New, B. (2000). Lifestyle medicines. British Medical Journal, $321,1341-1344$.

Glaser, B., \& Strauss, A. (1967). Discovery of grounded theory: Strategies for qualitative research. Chicago: Aldine Publishing Company.

Goetz, T. (2008, March 23). Practicing patients. Retrieved March 23, 2008, from http://www.nytimes.com/2008/03/23/magazine/23patientst.html? r=1\&scp=1\&sq=practicing + patients\&st=nyt\&oref=slogin

Goodare, H., \& Lockwood, S. (1999). Involving patients in clinical research: Improves the quality of research. British Medical Journal, 319(7212), 724-725.

Gooden, R., \& Winefield, H. (2007). Breast and prostate cancer online discussion boards: A thematic analysis of gender differences and similarities. Journal of Health Psychology, 12(1), 103-114.

Goudie, A., Cooper, G., \& Halford, J. (2004). Antipsychotic-induced weight gain. Diabetes, Obesity, and Metabolism, 7(5), 478-487.

Gray, R., Rofail, D., Allen, J., \& Newey, T. (2005). A survey of patient satisfaction with and subjective experiences of treatment with antipsychotic medication. Journal of Advanced Nursing, 52(1), 31-37.

Greene, J. (2004). Attention to 'Details': Etiquette and the pharmaceutical salesman in postwar America. Soc Stud Sci, 34(2), 271-292. 
Greenhill, L., Vitiello, B., Riddle, M., Fisher, P., Shockey, E., March, J., et al. (2003). Review of safety assessment methods used in pediatric psychopharmacology. $J$ Am Acad Child Adolesc Psychiatry, 42(6), 627-633.

Griffith, V. (n.d.). Wikipedia presentation: Berkeley. Retrieved May 30, 2008, from http://virgil.gr/1+Berkeley-WikiScanner_Presentation.ppt

Hardey, M. (2001). 'E-health': The Internet and the transformation of patients into consumers and producers of health knowledge. Information, Communication \& Society, 4(3), 388-405.

Hardey, M. (2002). 'The story of my illness': Personal accounts of illness on the Internet. Health, 6(1), 31-46.

Harmon, A. (2005, November 16). Young, assured and playing pharmacist to friends. The New York Times.

Harris, G. (2007, June 27). Psychiatrists top list in drug maker gifts. The New York Times Retrieved February 4, 2008, from http://www.nytimes.com/2007/06/27/health/psychology/27doctors.html\#

Harrison-Walker, L. (2001). E-complaining: A content analysis of an Internet complaint forum. Journal of Services Marketing, 15(5), 397-412.

Hauser, G. (1999). Vernacular voices: The rhetoric of publics and public spheres. Columbia: University of South Carolina.

Hayek, F. (1945). The use of knowledge in society. American Economic Review, 35(4), 519-530.

Healy, D. (2004). Shaping the intimate: Influences on the experience of everyday nerves. Soc Stud Sci, 34, 219-245.

Healy, D. (2006a). The latest mania: Selling bipolar disorder. PLoS Medicine, 3(4), 441444.

Healy, D. (2006b). Manufacturing consensus. Cult Med Psychiatry, 30, 135-156.

Heres, S., Davies, J., Maino, K., Jetzinger, E., Kissling, W., \& Leucht, S. (2006). Why olanzapine beats risperidone, risperidone beats quetiapine, and quetiapine beats olanzapine: An exploratory analysis of head-to-head comparison studies of second-generation antipsychotics. Am J Psychiatry, 163, 185-194.

Hicks, R., Wogalter, M., \& Vigilante, W. (2005). Placement of benefits and risks in prescription drug manufacturers' websites and information source expectations. Drug Information Journal, 39, 267-278. 
Hughes, S., \& Cohen, D. (2010). Understanding the assessment of psychotropic drug harms in clinical trials to improve social workers' role in medication monitoring. Social Work, 55(2), 105-115.

Huston, P., \& Moher, D. (1996). Redundancy, disaggregation, and the integrity of medical research. Lancet, 347(9007), 1024-1027.

Hyden, L. (1997). Illness and narrative. Sociology of Health \& Illness, 19(1), 48-69.

Ioannidis, I., \& Lau, J. (2001). Completeness of safety reporting in randomized trials: An evaluation of 7 medical areas. JAMA, 285, 437-443.

Jacobs, D., \& Cohen, D. (1999). What is really known about psychological alterations produced by psychiatric drugs? International Journal of Risk \& Safety in Medicine, 12, 37-47.

Kazdin, A. (1999). The meanings and measurement of clinical significance. Journal of Consulting and Clinical Psychology, 67(3), 332-339.

Keen, A. (2007). The cult of the amateur: How today's internet is killing our culture. New York: Doubleday.

Kesselheim, A., \& Avorn, J. (2007). The role of litigation in defining drug risks. JAMA, 297(3), 308-311.

Kessler, D. (1993). Introducing MEDWatch: A new approach to reporting medication and device adverse effects and product problems. JAMA, 269(21), 2765-2768.

Kirsch, I., Moore, T., Scoboria, A., \& Nicholls, S. (2002). The Emperor's new drugs: An analysis of antidepressant medication data submitted to the U.S. Food and Drug Administration. Prevention and Treatment, 5, article 23.

Kivits, J. (2004). Researching the 'informed patient': The case of online health information. Information, Communication \& Society, 7(4), 510-530.

Kravitz, R., Epstein, R., Feldman, M., Franz, C., Azari, R., Wilkes, M., et al. (2005). Influence on patients' requests for direct-to-consumer advertised antidepressants: A randomized controlled trial. JAMA, 293(16), 1995-2002.

Krippendorff, K. (2004). Content analysis: An introduction to its methodology (2nd ed.). Thousand Oaks: SAGE Publications.

Kutchins, H., \& Kirk, S. (1997). Making us crazy: DSM, the psychiatric bible and the creation of mental disorders. New York: The Free Press. 
Lagomasino, I., Dwight-Johnson, M., \& Simpson, G. (2005). The need for effectiveness trials to inform evidence-based psychiatric practice. Psychiatr Serv, 56, 649-651.

Lakoff, A. (2004). The anxieties of globalization: Antidepressant sales and economic crisis in Argentina. Soc Stud Sci, 34(2), 247-269.

Lasalvia, A., Ruggeri, M., \& Santolini, N. (2002). Subjective quality of life: Its relationship with clinician-rated and patient-rated psychopathology. Psychother Psychosom, 71, 275-284.

Leslie, D., Mohamed, S., \& Rosenheck, R. (2009). Off-label use of antipsychotic medications in the department of veterans affairs health care system. Psychiatric Services, 60, 1175-1181.

Let data speak to data. (2005). Nature, 438(7068), 531.

Lewis, T. (2006). Seeking health information on the internet: Lifestyle choice or bad attack of cyberchondria? Media, Culture \& Society, 28(4), 521-539.

Lexchin, J. (1993). Interactions between physicians and the pharmaceutical industry: What does the literature say? Can Med Assoc J, 149(10), 1401-1407.

Lexchin, J. (2006). Bigger and better: How Pfizer redefined erectile dysfuntion PLoS Medicine, 3(4), 429.

Lexchin, J., Bero, L., Djulbegovic, B., \& Clark, O. (2003). Pharmaceutical industry sponsorship and research outcome and quality: A systematic review. British Medical Journal, 326(7400), 1167-1171.

Macias, W., \& Lewis, L. (2003). A content analysis of direct-to-consumer prescription drug web sites. Journal of Advertising, 32(4), 43-56.

Markham, A. (2004). Internet communication as a tool for qualitative research. In D. Silverman (Ed.), Qualitative research: Theory, methods, and practice (2nd ed., pp. 95-123). Thousand Oaks: SAGE Publications.

McCubbin, M., \& Cohen, D. (1996). Extremely unbalanced: Interest divergence and power disparities between clients and psychiatry. International Journal of Law and Psychiatry, 19(1), 1-25.

McCurdy, D. (2003, February 1). Drug culture supermodels of the medicine cabinet. The Dominion Post.

Medawar, C., \& Herxheimer, A. (2003). A comparison of adverse drug reaction reports from professionals and users, relating to risk of dependence and suicidal behavior with paroxetine. International Journal of Risk \& Safety in Medicine, 16, 5-19. 
Medawar, C., Herxheimer, A., Bell, A., \& Jofre, S. (2002). Paroxetine, Panorama, and user reporting of ADRs: Consumer intelligence matters in clinical practice and post-marketing drug surveillance. International Journal of Risk \& Safety in Medicine, 15, 161-169.

Melander, H., Ahlqvist-Rastad, J., Meijer, G., \& Beermann, B. (2003). Evidence-b(i)ased medicine - selective reporting from studies sponsored by pharmaceutical industry: Review of studies in new drug applications. Br Med J, 326, 1171-1173.

Miller, W., \& Crabtree, B. (2005). Clinical research. In N. Denzin \& Y. Lincoln (Eds.), The sage handbook of qualitative research (pp. 605-639). Thousand Oaks: Sage Publications.

Miracle drugs or media drugs? (1992, March). Consum Rep, 57(3), 142.

Mitchell, T. (2002). Rule of experts: Egypt, techno-politics, modernity. Berkeley: University of California Press.

Moncrieff, J. (2001). Are antidepressants overrated? A review of methodological problems in antidepressant trials. J Nerv Ment Dis, 189, 288-295.

Moncrieff, J. \& Cohen, D. (2006). Do antidepressants cure or create abnormal brain states? PLoS Medicine, 3(7), e240.

Moncrieff, J., \& Thomas, P. (2002). Psychiatry should not accept so much commerical sponsorship. British Medical Journal, 325, 216.

Moore, L., \& Clarke, A. (2001). The traffic in cyberanatomies: sex/gender/sexualities in local and global formations. Body \& Society, 7(1), 57-96.

Moynihan, R., Bero, L., Ross-Degnan, D., Henry, D., Lee, K., Watkins, J., et al. (2000). Coverage by the news media of the benefits and risks of medications. $N$ Engl J Med, 342, 1645-1650.

Murphy, S., \& Roberts, R. (2006). "Black box" 101: How the Food and Drug Administration evaluates, communicates, and manages drug benefit/risk. $J$ Allergy Clin Immunol, 117, 34-39.

Nordin, I. (2000). Expert and non-expert knowledge in medical practice. Medicine, Health Care and Philosophy, 3(3), 297-304.

O'Neill, A. (2007). The patient trust deficit in pharmaceutical marketing. DTC Perspectives, December, 12-16. 
Oates, J. (2006). The science of drug therapy. In L. Brunton, J. Lazo \& K. Parker (Eds.), Goodman \& Gilman's the pharmacological basis of therapeutics (11 ed.). New York: McGraw-Hill.

Papanikolaou, P., Churchill, R., Wahlbeck, K., \& Ioannidis, I. (2004). Safety reporting in randomized trials of mental health interventions. Am J Psychiatry, 161, 16921697.

Papanikolaou, P., \& Ioannidis, J. (2003). Awareness of the side effects of possessed medications in a community setting. Eur J Clin Pharmacol, 58, 821-827.

Parr, H. (2002). New body-geographies: the embodied spaces of health and medical information on the Internet. Environment and Planning D: Society and Space, 20, 73-95.

Perlis, R., Perlis, C., Wu, Y., Hwang, C., Joseph, M., \& Nierenberg, A. (2005). Industry sponsorship and financial conflict of interest in the reporting of clinical trials in psychiatry. Am J Psychiatry, 162(10), 1957-1960.

Peterson, A., \& Bunton, R. (Eds.). (1997). Foucault, health and medicine. New York: Routledge.

Philip, N., Mello, K., Carpenter, L., Tyrka, A., \& Price, L. (2008). Patterns of quetiapine use in psychiatric inpatients: An examination of off-label use. Annals of Clinical Psychiatry, 20(1), 15-20.

Phillips, D., Kanter, E., Bednarczyk, B., \& Tastad, P. (1991). Importance of the lay press in the transmission of medical knowledge to the scientific community. $N$ Engl $J$ Med, 325, 1180-1183.

Popay, J., \& Williams, G. (1996). Public health research and lay knowledge. Social Science \& Medicine, 42(5), 759-768.

Powell, J., McCarthy, N., \& Eysenbach, G. (2003). Cross-sectional survey of users of Internet depression communities. BMC Psychiatry Retrieved July 15, 2007, from http://www.biomedcentral.com/1471-244X/3/19

Prior, L. (2003). Belief, knowledge and expertise: The emergence of the lay expert in medical sociology. Sociology of Health \& Illness, 25, 41-57.

Rabinow, P. (Ed.). (1984). The Foucault reader. New York: Pantheon Books.

Radley, D., Finkelstein, S., \& Stafford, R. (2006). Off-label prescribing among officebased physicians. Archives of Internal Medicine, 166, 1021-1026. 
Rawlinson, M. (1987). Foucault's strategy: Knowledge, power, and the specificity of truth. The Journal of Medicine and Philosophy, 12, 371-395.

Read, J. (2008). Schizophrenia, drug companies and the internet. Social Science \& Medicine, 66, 99-109.

Relman, A., \& Angell, M. (2002). America's other drug problem. The New Republic, $227(25), 27-41$.

Rogers, R. (2003). The Viagra files: The web as anticipatory medium. Prometheus, 21(2), 195-211.

Rogers, R. (2004). Behind the practice of information politics. In R. Rogers (Ed.), Information politics on the Web. Cambridge, MA: MIT Press.

Rogers, R., \& Marres, N. (2000). Landscaping climate change: A mapping technique for understanding science and technology debates on the World Wide Web. Public Understand. Sci, 9, 1-23.

Rogers, R., \& Zelman, A. (2002). Surfing for knowledge in the information society. In G. Elmer (Ed.), Critical perspectives on the Internet. Lanham, MD: Rowman \& Littlefield.

Rose, N. (1993). Government, authority and expertise in advanced liberalism. Economy and Society, 22(3), 283-299.

Schonfeld, E., \& Morrison, C. (2007). The next disrupters. Retrieved May 22, 2008, from http://money.cnn.com/magazines/business2/business2 archive/2007/09/01/10016 9862/index.htm

Schroder, S., Zollner, Y., \& Schaefer, M. (2007). Drug related problems with Antiparkinsonian agents: Consumer Internet reports versus published data. Pharmacoepidemiology and Drug Safety, 16, 1161-1166.

Siegel, L. (2008). Against the machine: Being human in the age of the electronic mob. New York: Spiegel \& Grau.

Sillence, E., Briggs, P., Harris, P., \& Fishwick, L. (2007). Health websites that people can trust - the case of hypertension. Interacting with computers, 19, 32-42.

Sismondo, S. (2007). Ghost management: How much of the medical literature is shaped behind the scenes by the pharmaceutical industry? PLoS Medicine, 4(9), 14291433. 
Skitka, L., \& Sargis, E. (2006). The internet as psychological laboratory. Annual Review of Psychology, 57, 529-555.

Smarty, S., \& Findling, R. (2007). Psychopharmacology of pediatric bipolar disorder: A review. Psychopharmacology, 191, 39-54.

Smith, R. (2005). Medical journals are an extension of the marketing arm of pharmaceutical companies. PLoS Medicine, 2(5), 364-366.

Stone, S. (2007). Patient concerns posthaemmorrhagic stroke: a study of the Internet narratives of patients with ruptured arteriovenus malformation. Journal of Clinical Nursing, 16, 289-297.

Stowell, K., Ghinassi, F., Fabian, T., Nash, K., \& Haskett, R. (2009). An intervention to promote evidence-based prescribing at a large psychiatric hospital. Psychiatric Services, 60, 294-296.

Tapscott, D., \& Williams, A. (2006). Wikinomics: How mass collaboration changes everythin. New York: Penguin Group.

Taylor, H., \& Leitman, R. (2002). Cyberchondriacs continue to grow in America. Retrieved July 13, 2007, from http://www.harrisinteractive.com/news/newsletters/healthnews/HI_HealthCareNe ws2002Vol2_Iss09.pdf

Taylor, D., \& McAskill, R. (2000). Atypical antipsychotics and weight gain - a systematic review. Acta Psychiatrica Scandinavica, 101(6), 416-432.

Thomaselli, R. (2007, June 4). Big pharma doesn't like how it looks on YouTube. Advertising Age.

Titscher, S., Meyer, M., Wodak, R., \& Vetter, E. (2000). Methods of text and discourse analysis (B. Jenner, Trans.). Thousand Oaks: SAGE Publications.

Today in eHealth news: Heard on the street. (2007, January 4). Retrieved February 1, 2008, from

http://www.manhattanresearch.com/newsroom/Recent_Coverage/01042007_AIS $\underline{\text { Health.aspx }}$

Top 10 products by U.S. dispensed prescriptions. (2007). Retrieved February 1, 2008, from http://www.imshealth.com/ims/portal/front/articleC/0,2777,6599_80411799_8041 $\underline{3615,00 . h t m l}$ 
Top 10 products by U.S. sales. (2007). Retrieved February 1, 2008, from http://www.imshealth.com/ims/portal/front/articleC/0,2777,6599_80408845_8041 $\underline{1835,00 . \mathrm{html}}$

Trickett, E. (2007, May 28). Information excess is healthy challenge for pharma PR pros. PR Week.

Tucker, G. (1998). Putting DSM-IV in perspective. American Journal of Psychiatry, 155(2), 159-161.

Turner, E., Matthews, A., Linardatos, E., Tell, R., \& Rosenthal, R. (2008). Selective publication of antidepressant trials and its influence on apparent efficacy. $N$ Engl J Med, 358(3), 252-260.

Turner, S. (2001). What is the problem with experts? Soc Stud Sci, 31(1), 123-149.

Von Knoop, C., Lovich, D., Silverstein, M., \& Tutty, M. (2003). Vital signs: E-health in the United States. Retrieved February 1, 2008, from http://www.bcg.com/impact expertise/publications/files/Vital_Signs_E_Health_U nited_States_Jan2003.pdf

Waack, K., Ernst, M., \& Graber, M. (2004). Informational content of official pharmaceutical industry websites about treatments for erectile dysfunction. The Annals of Pharmacotherapy, 38, 2029-2034.

Wales, J. (2005). The intelligence of wikipedia. Retrieved May 5, 2008, from http://webcast.oii.ox.ac.uk/?view=Webcast\&ID $=20050711 \_76$

Warnick, B. (2007). Rhetoric online: Persuasion and politics on the world wide web. New York: Peter Lang.

Wazana, A. (2000). Physicians and the pharmaceutical industry: Is a gift ever just a gift? JAMA, 283(3), 373-380.

Weare, C., \& Lin, W. (2000). Content analysis of the world wide web: Opportunities and challenges. Social Science Computer Review, 18(3), 272-292.

Weiss, M., \& Fitzpatrick, R. (1997). Challenges to medicine: The case of prescribing. Sociology of Health \& Illness, 19(3), 297-327.

Wicks, P. (2008). ALS patients: Give us the truth about cognitive change. Retrieved May 22, 2008, from http://blog.patientslikeme.com/category/research

Williams, D. (2007). PatientsLikeMe receives its first scientific award. Retrieved May 22, 2008, from http://blog.patientslikeme.com/category/research 
Williams, S., \& Calnan, M. (1996). Modern medicine: Lay perspectives and experiences. London: UCL Press.

Woloshin, S., \& Schwartz, L. (2002). Press releases: Translating research into news. JAMA, 287(21), 2856-2858.

Woosley, R. (1994). A prescription for better prescriptions. Issues Sci Technol, 10(3), 5967.

Wyatt, J. (1991). Use and sources of medical knowledge. Lancet, 338(8779), 1368-1374.

Zinberg, N. (1986). Drug, Set, and Setting: The Basis for Controlled Intoxicant Use. Yale University Press. 


\section{APPENDIX A}

Screen Shots of the 5 Selected Websites

Figure A1. Homepage on AskAPatient.com

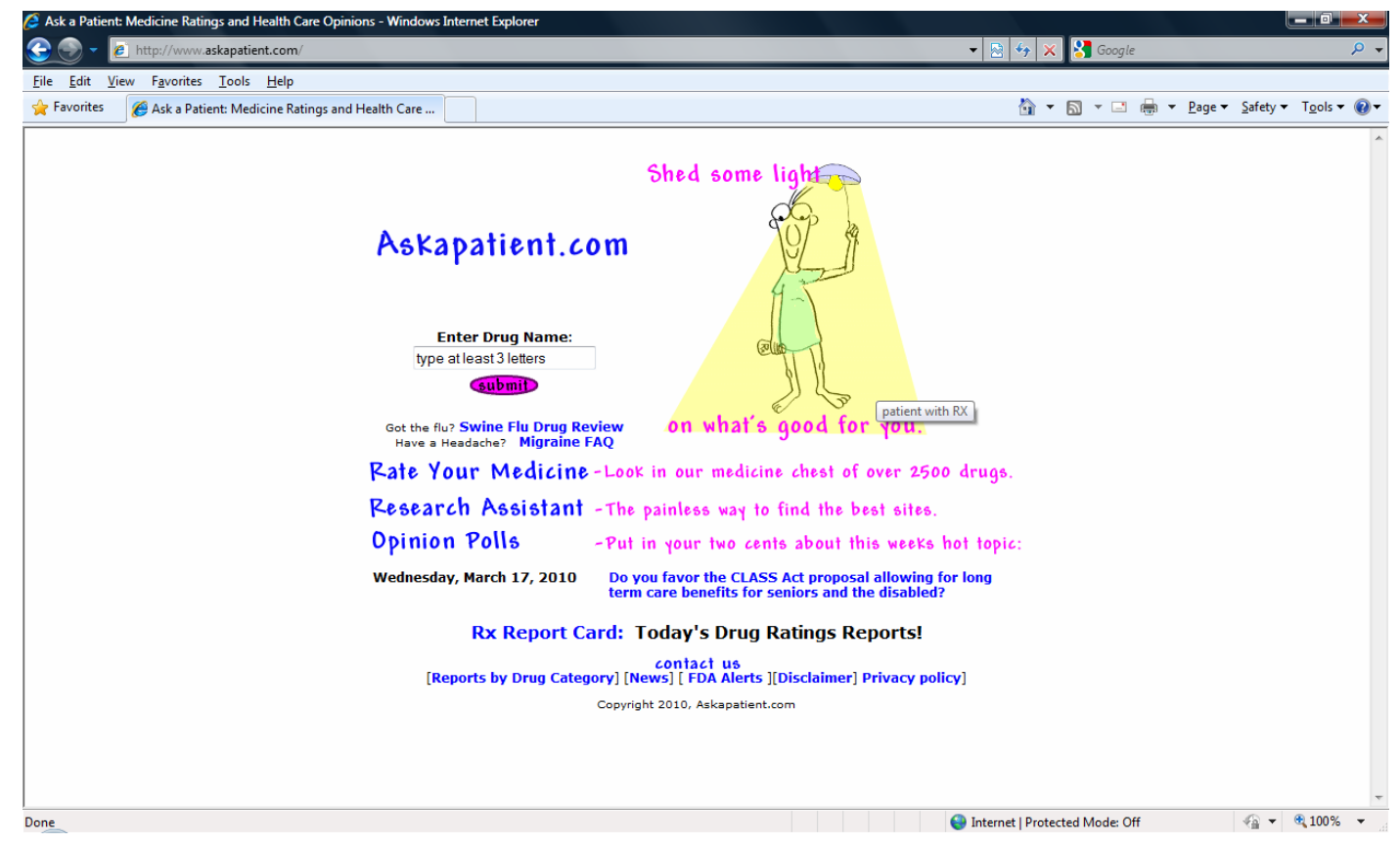




\section{Figure A2. Lexapro page on AskAPatient.com}

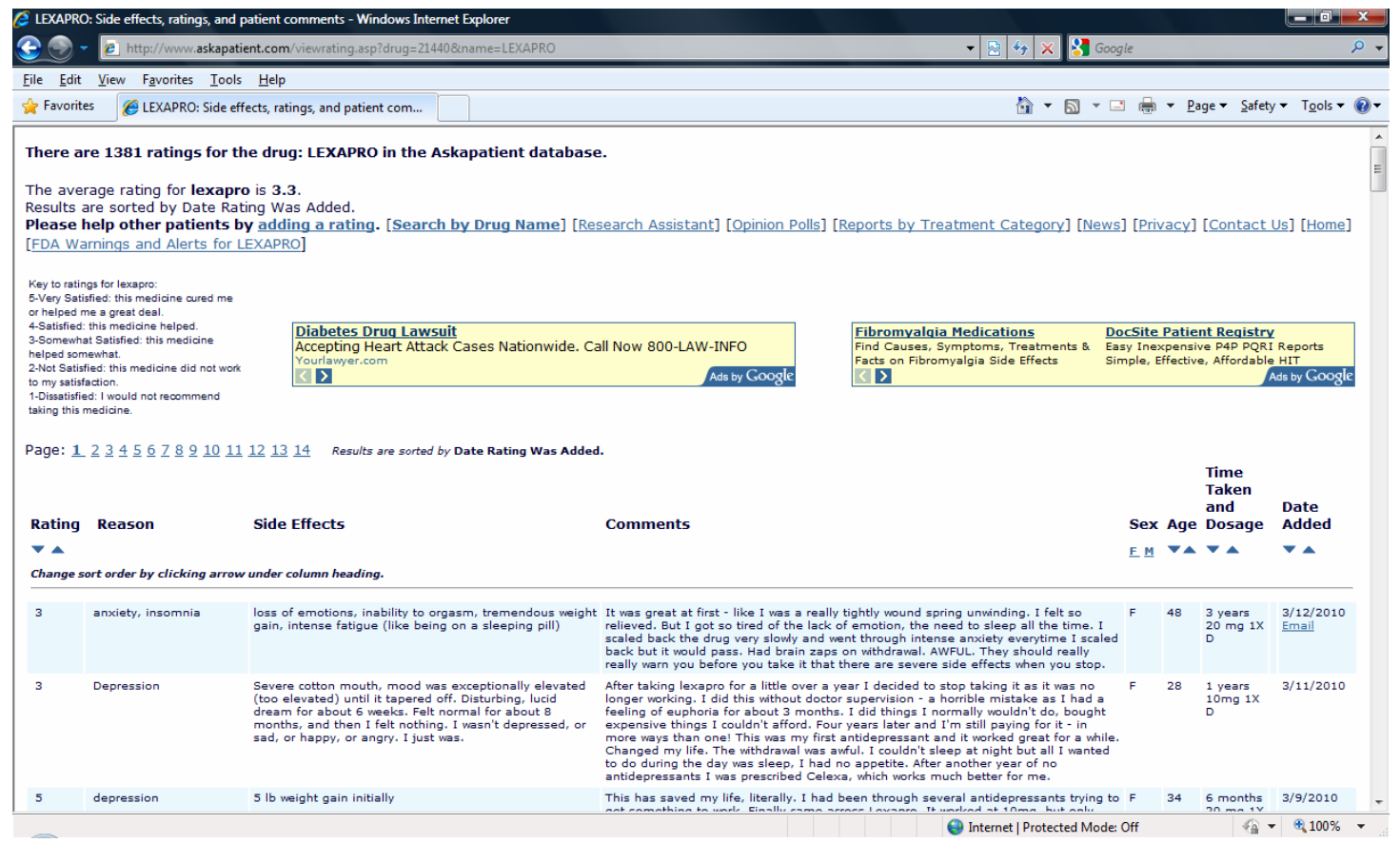

\section{Figure A3. Homepage on CrazyMeds.us}

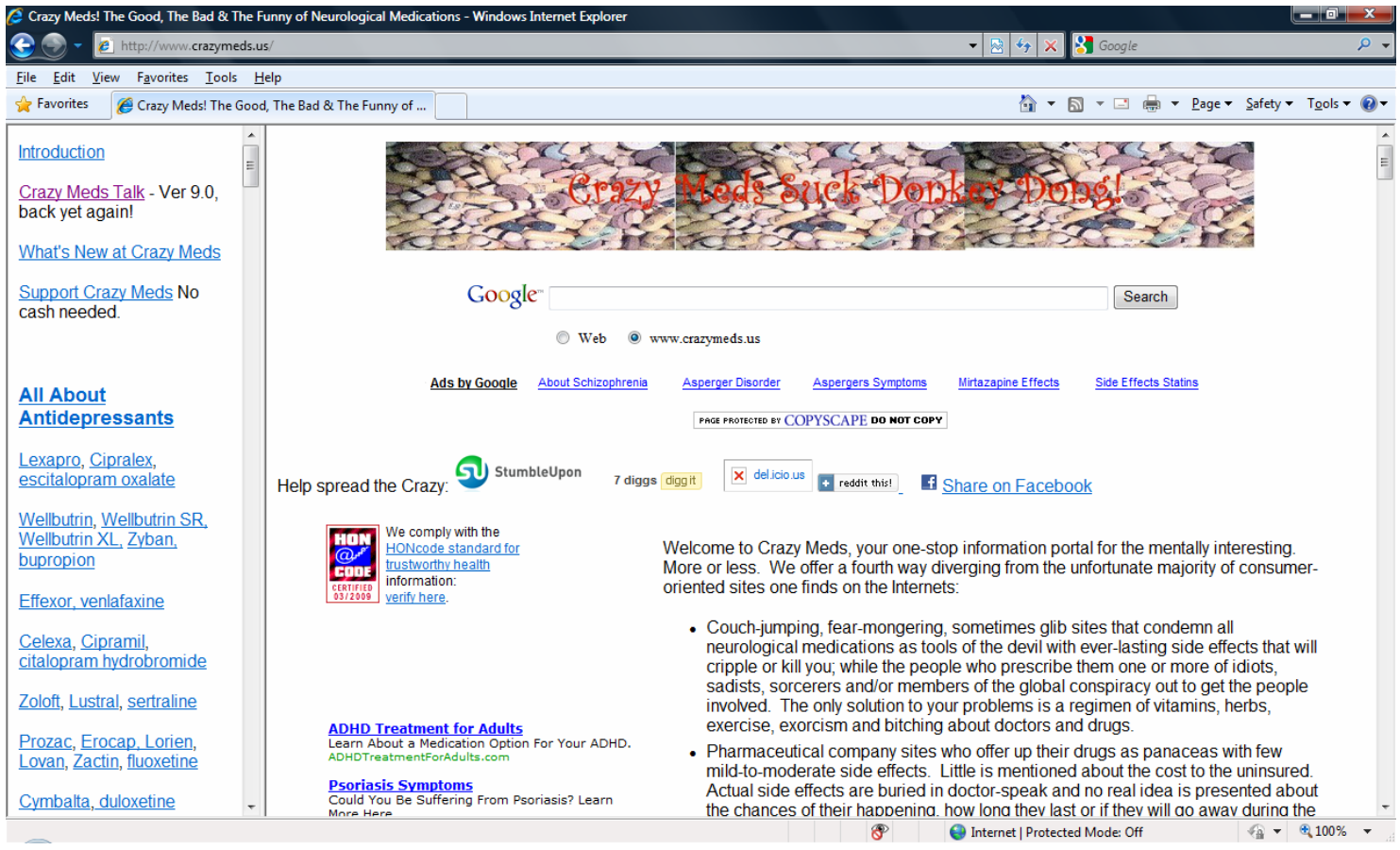


Figure A4. Lexapro page on CrazyMeds.us

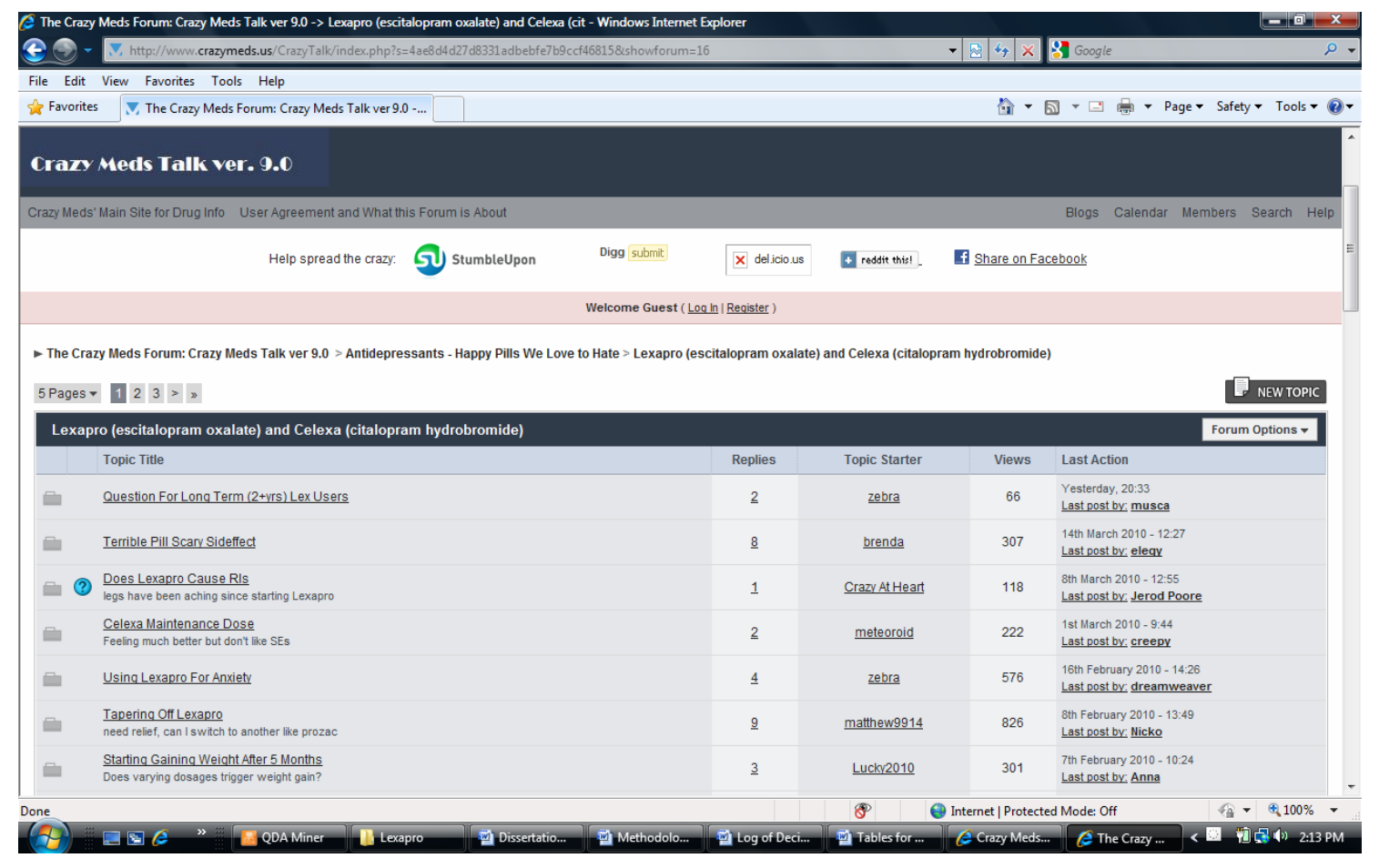


Figure A5. Homepage on TheIcarusProject.net

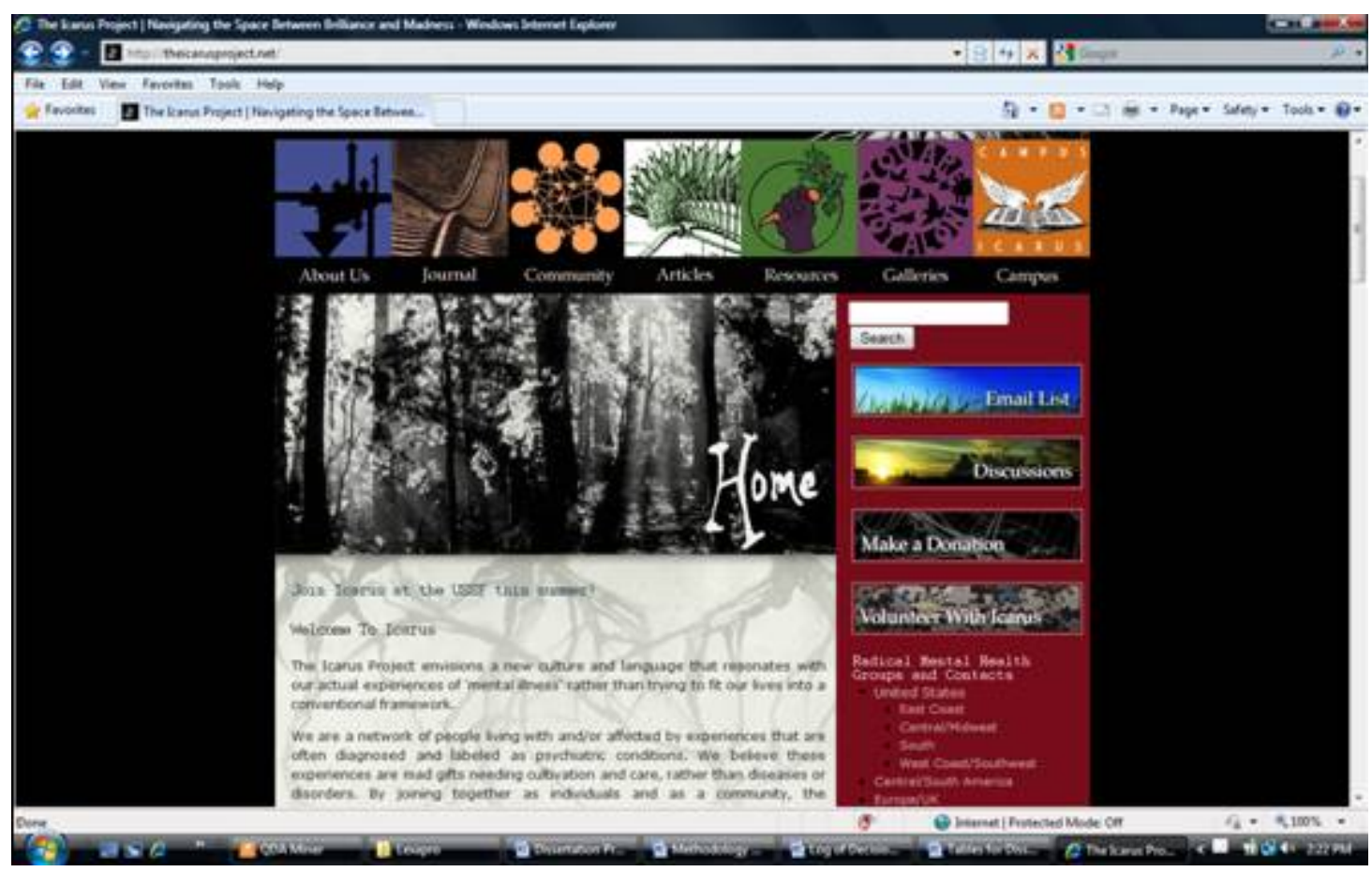

Figure A6. Medication page on TheIcarusProject.net

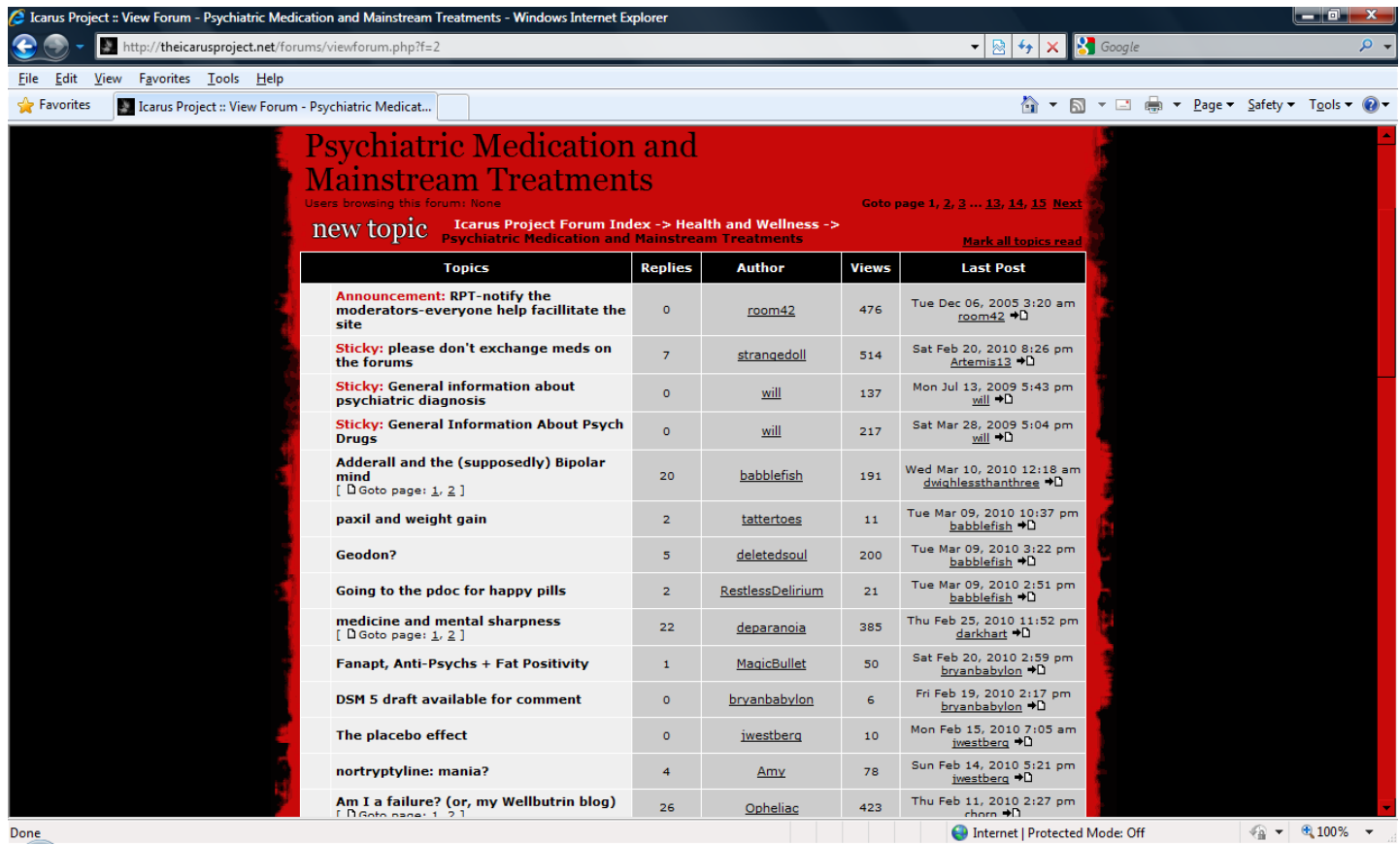


Figure A7. Homepage on WebMD.com

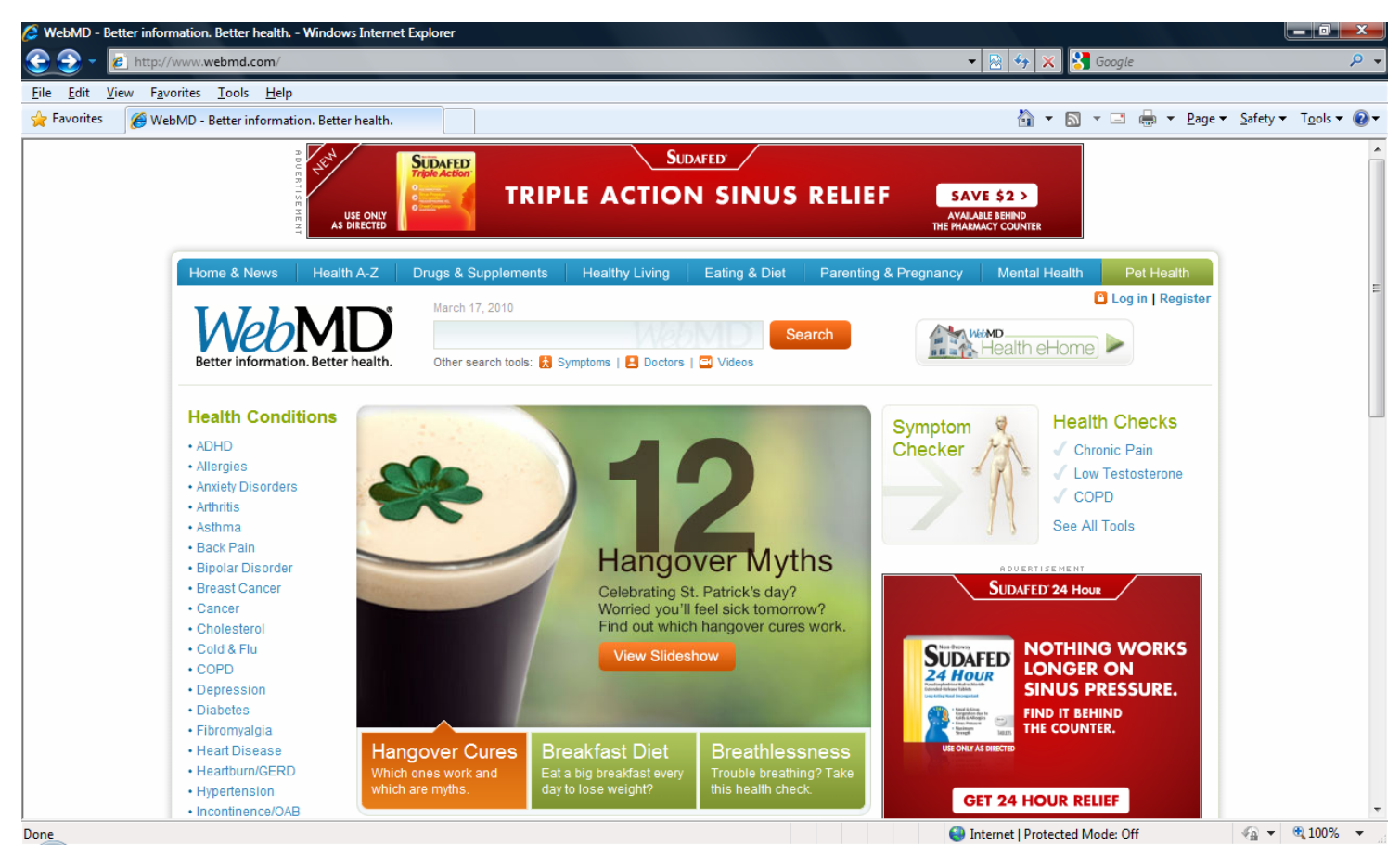


Figure A8. Lexapro page on WebMD.com

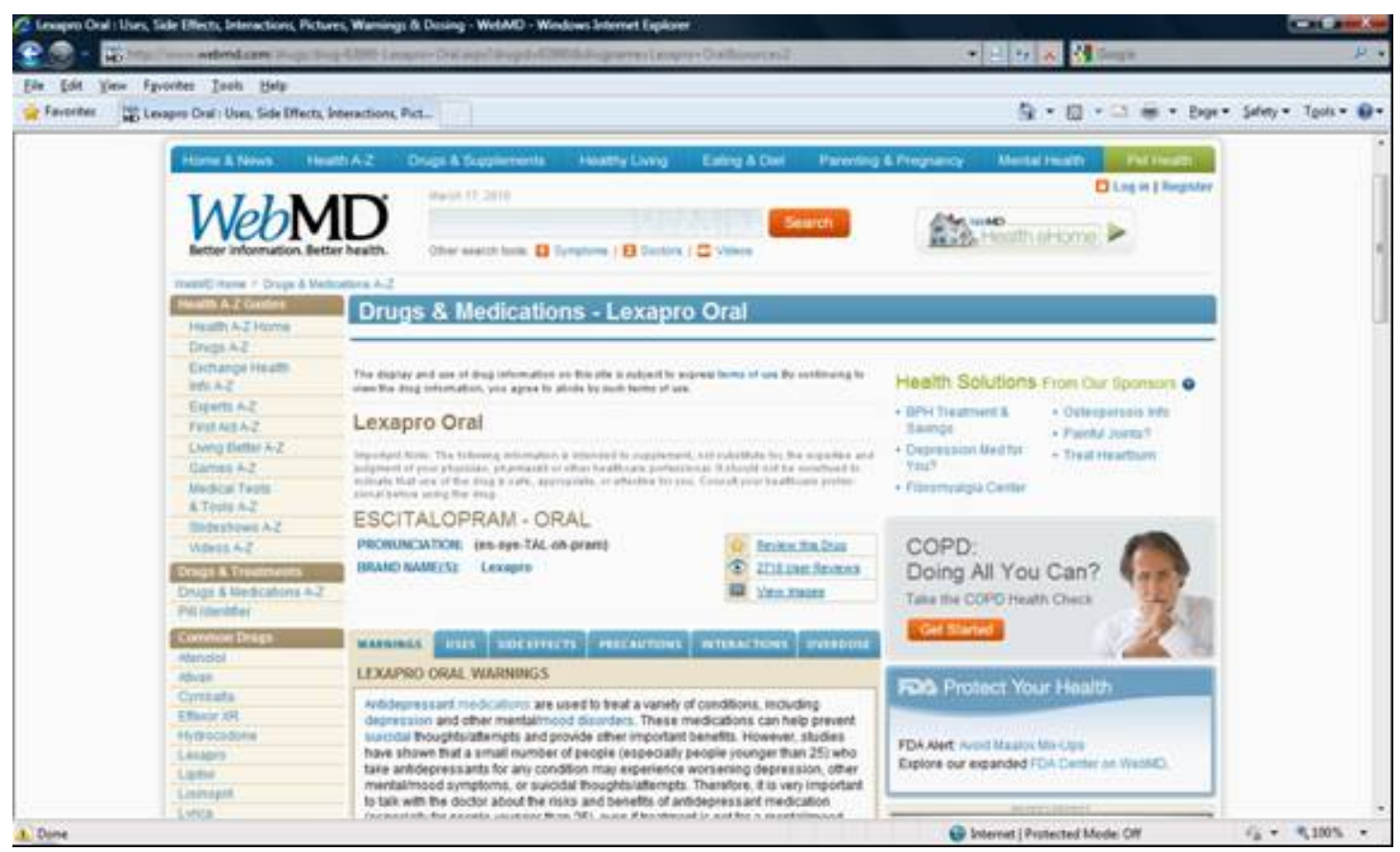


Figure A9. Lexapro user ratings/reviews on WebMD.com

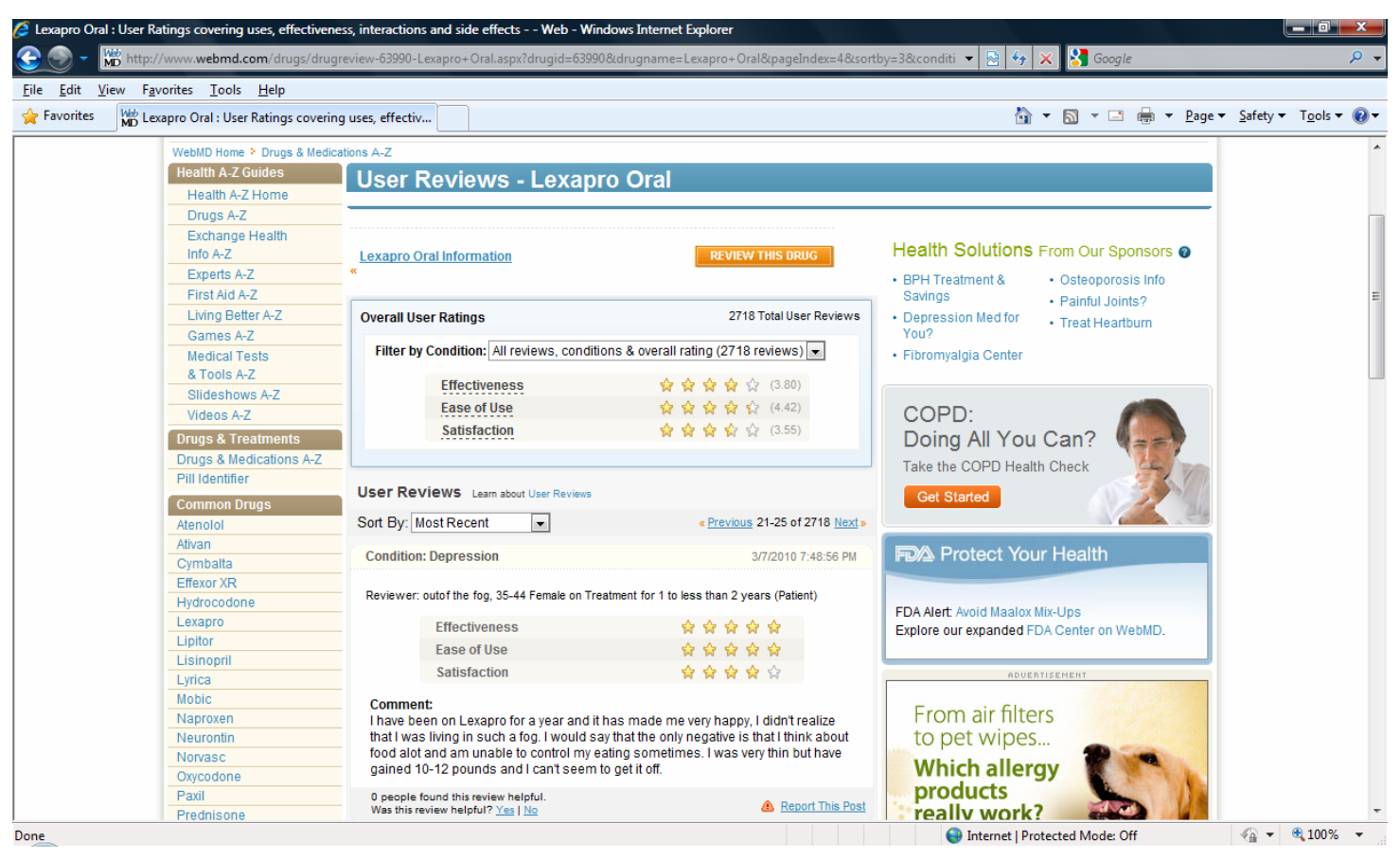


Figure A10. Homepage on RevolutionHealth.com

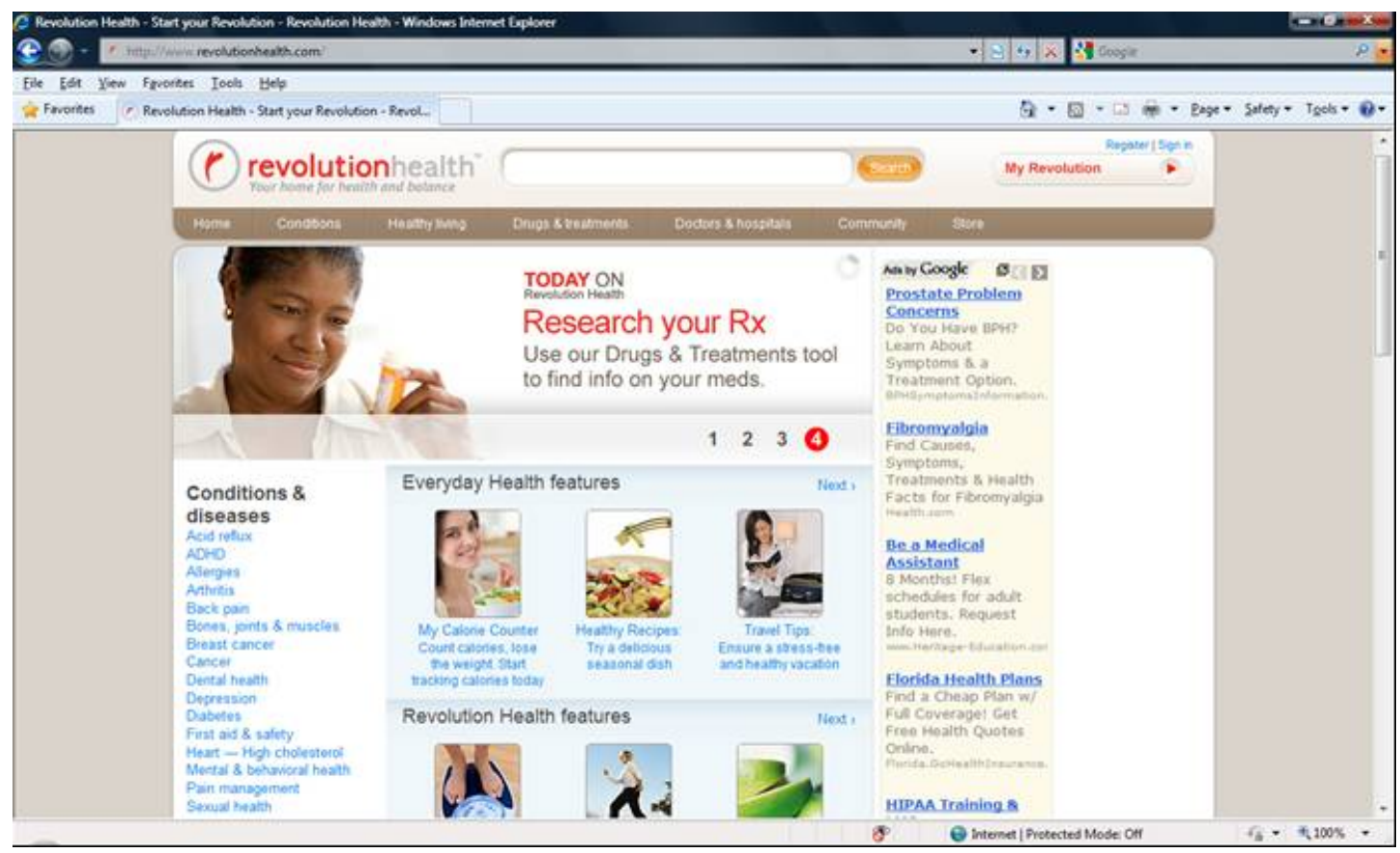


Figure A11. Lexapro page on RevolutionHealth.com

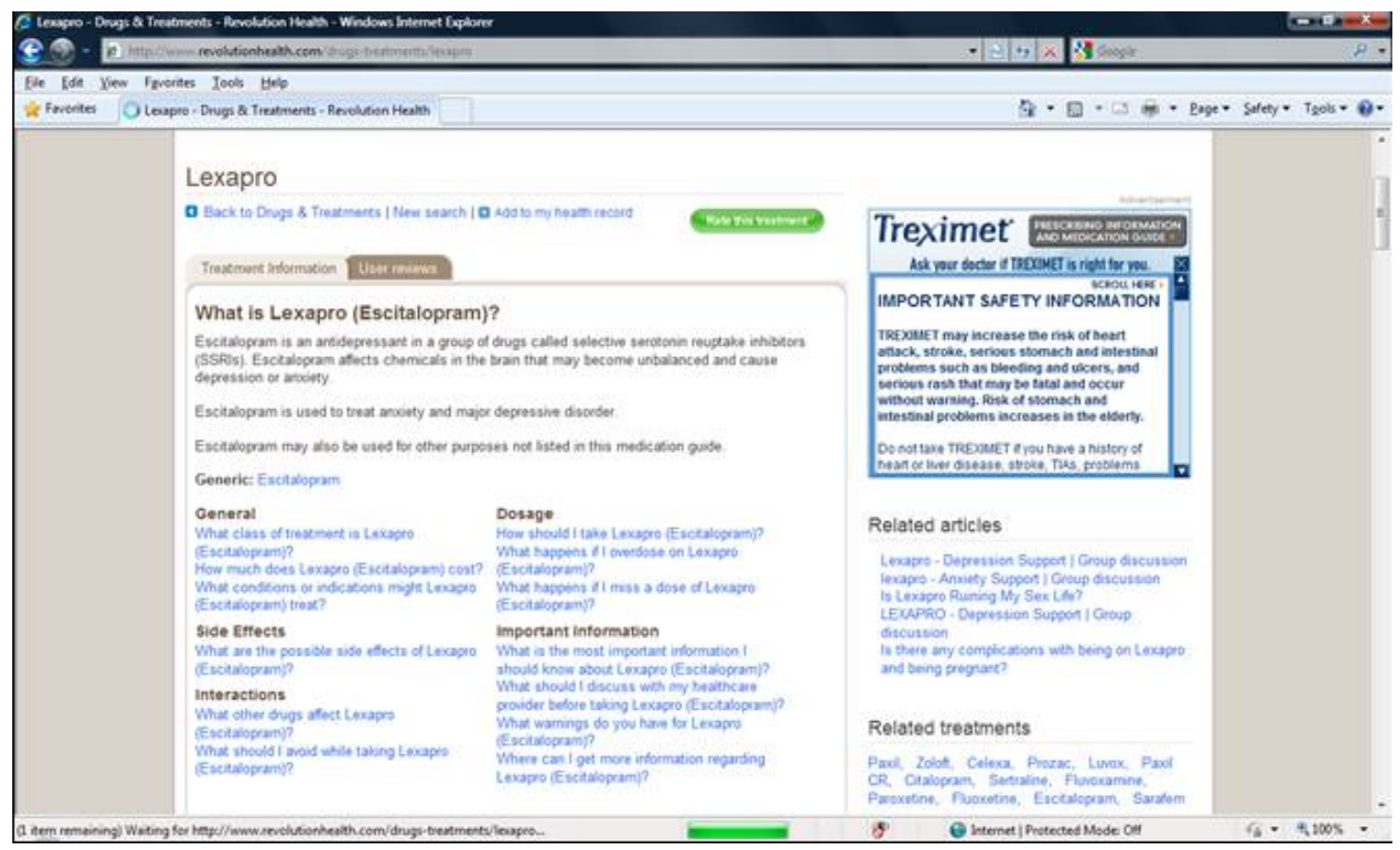


Figure A12. Lexapro user ratings/reviews on RevolutionHealth.com

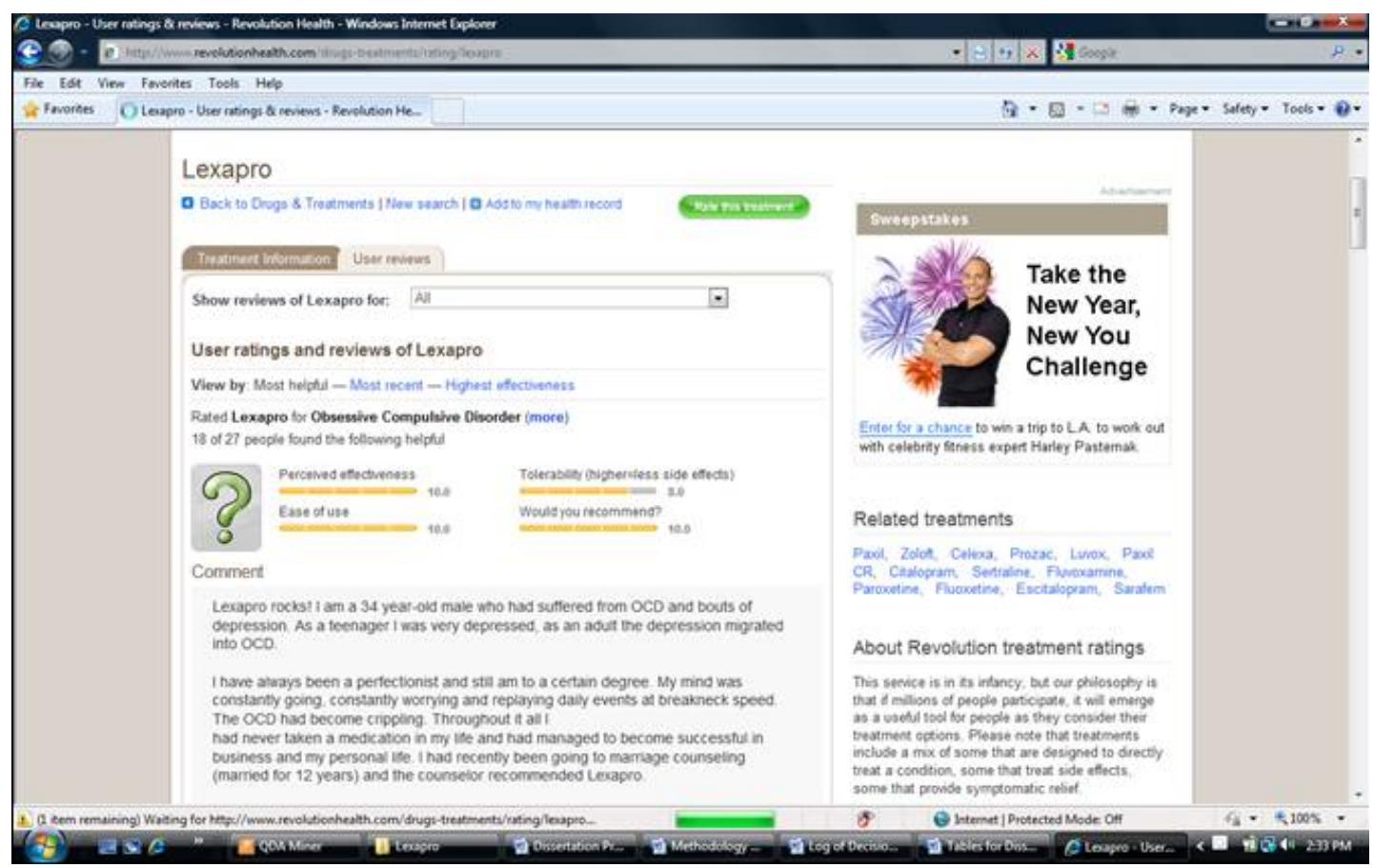




\section{APPENDIX B}

QDA Miner 3.2 Screen Shots of Coded Data

Figure B1.

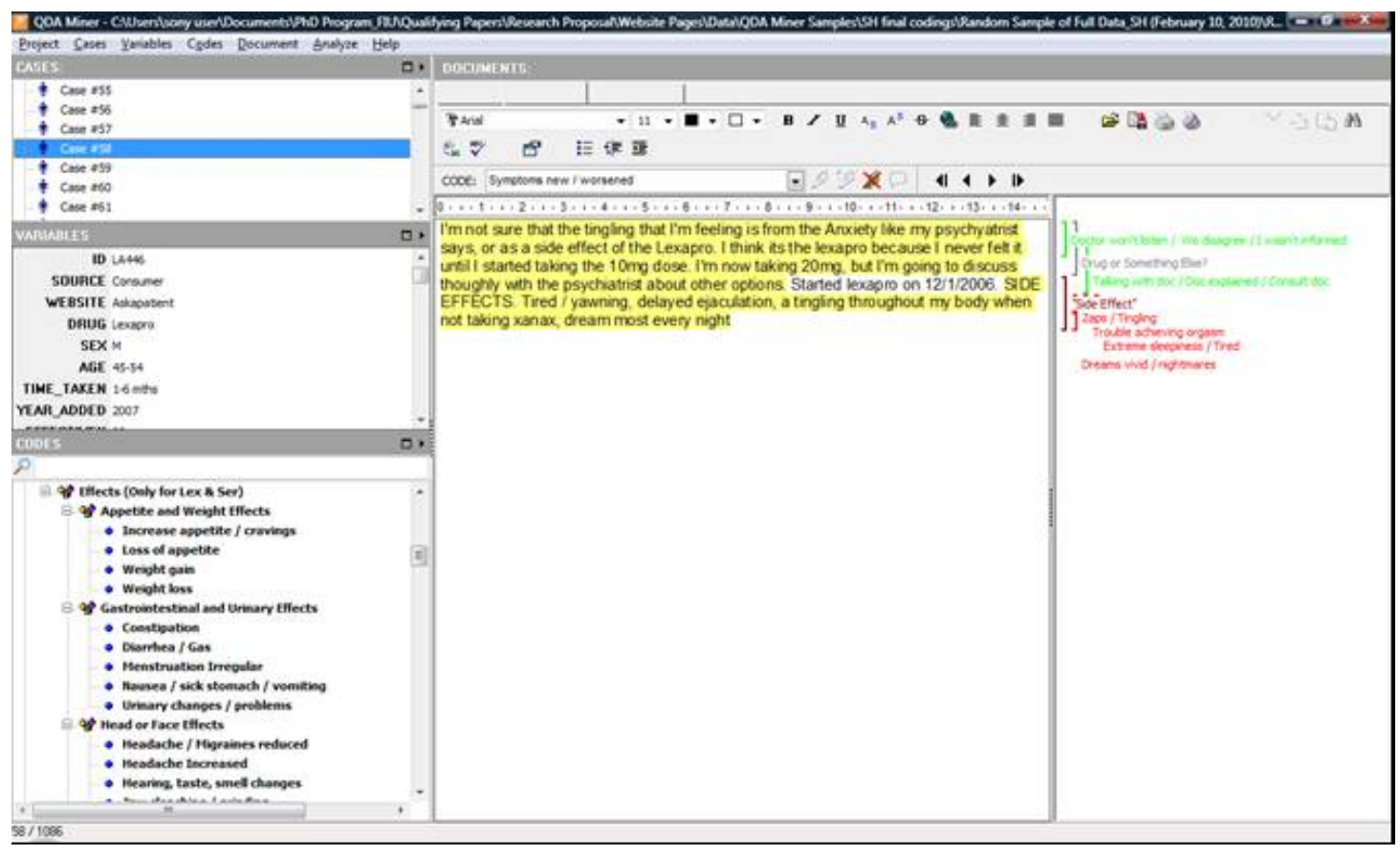




\section{Figure B2.}

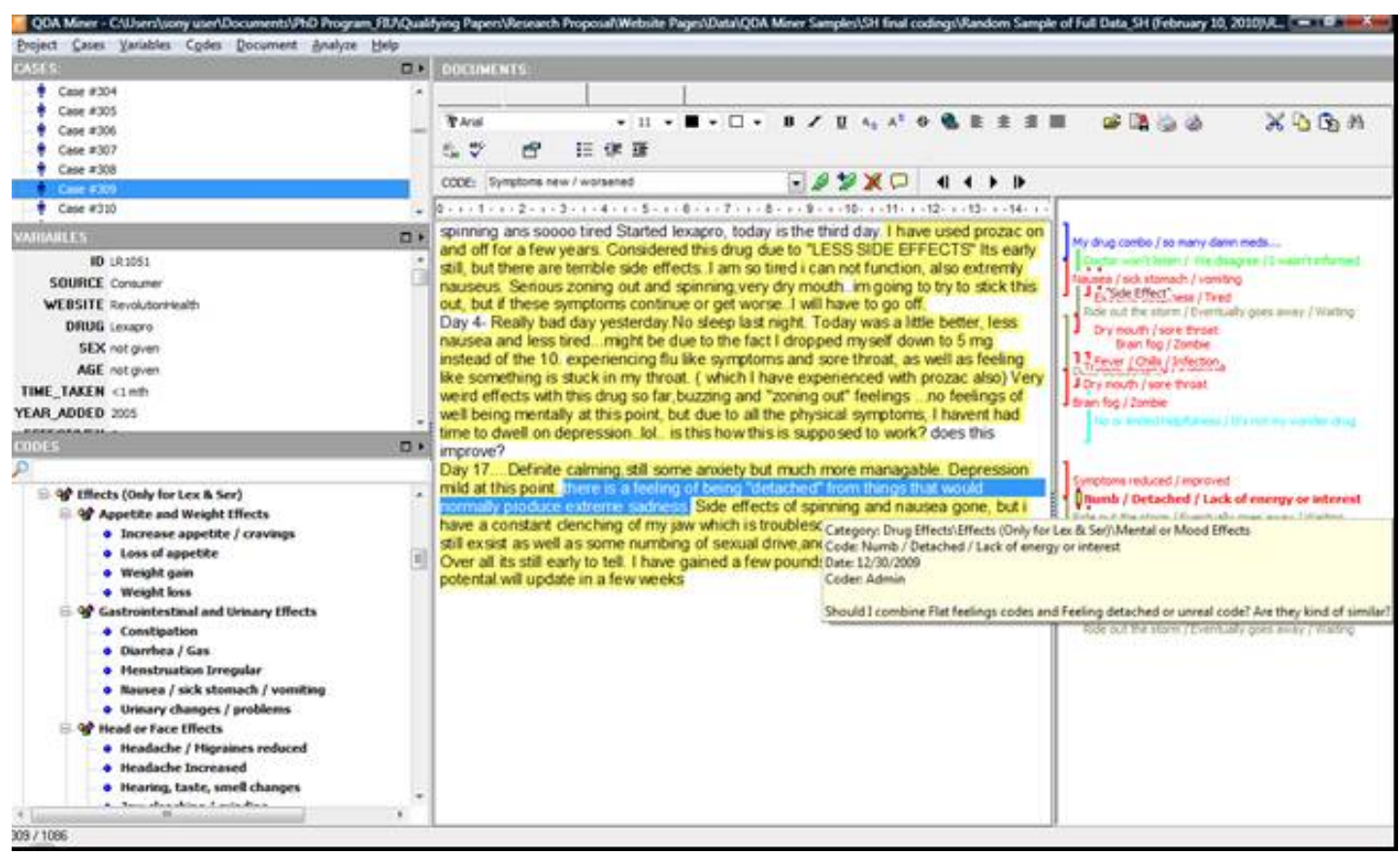

\section{Figure B3.}

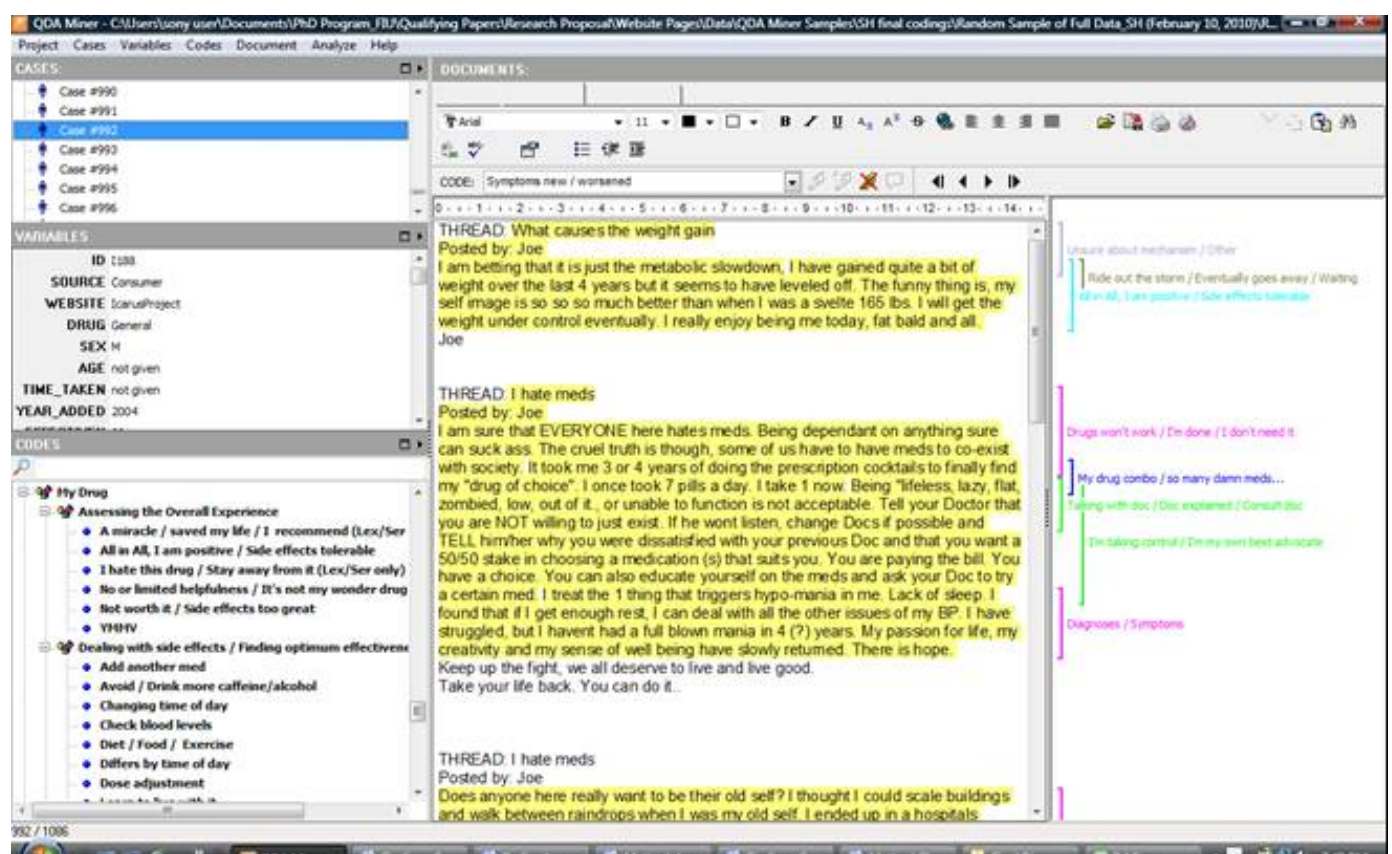




\section{APPENDIX C}

Initial Codebook: September 2009

- CITATIONS AND LINKS

o Academic Journal Internet

- DRUG EFFECTS

oChanges for the Better/Favorite or Best

Effects

- Able to live my life

- The world is good again

oChanges for the worse/"Worst" side effects

- I feel fat now

- Sex used to be part of my life

$\circ$ Consequences of Side Effects

- A disease for the rest of my life

- Can ruin my day

- Frightened

- Spun into mania by ADs/ rediagnosed manic/bipolar

- Unable to work/missing work

○Discontinuing / Missed Doses

- "withdrawals"

- Depression coming back (Disc)

- Diarrhea (Disc)

- Dizziness (Disc)

- Feel like a druggie needing a fix (Disc)

- Feel sick (Disc)

- Hallucinations (real and frightening) (Disc)

- Headache (Disc)

\section{oGeneral Beneficial Effects}

- Ability to do things in absence of symptoms

- Absence of specific symptoms

- Clear thinking/Less negative thoughts

- Irritability (Disc)

- Making me crazier than before (Disc)

- Passing out (Disc)

- Trouble sleeping (Disc)

- Visual tracers (Disc)

- Vomiting/nausea (Disc)

- Physically relaxed

- Pleasant / fun dreams

- Prevent/decrease mood swings/mania

- Reduced/eradicated anxiety

- Reduced anger

- Relatively normal / feel better

- Relief from problems/can handle stress

- Comfortable/At ease with myself/life

- Decrease hallucinations

- Depression lifted/ Smile and laugh more

- Emotionally stable / appropriate

- Energized 
- Helps me sleep

- Improved concentration

\section{○ General Effects Not Specified}

- Knocked me out

○General Side Effects

- Agitation/jump out of my skin

- Bad taste in mouth

- Carb cravings

- Constipation

- Crazy, vivid dreams/nightmares

- Depressed/feeling down/worsened depression

- Diabetes

- Difficult to wake up

- Difficulty focusing/Confused/Fuzzy

- Discomfort

- Dizzy

- Dry/sore eyes

- Dry mouth

- Dyskinesia

- Excessive sleeping

- Feel "high"

- Feel worthless

- Flat feelings/no emotions

- Gassy

- Groggy/Foggy

- Hangover feeling

- Headache

- Heaviness/soreness/pain/numbness in legs

- High blood pressure

- Impossible to get aroused

- Inability to reach orgasm

- Increase appetite

- Irregular menstruation

- Jaw clenching

- Jitteriness/"spazzy freak"

- Light headed

- Loss of appetite

- Lost sex drive/decreased libido

- Memory loss

No Beneficial Effects

○ No Effect On...

- Nasal congestion/snoring

- Nausea/sick stomach

- No interest in life

- No/diminished energy

- Not sleeping well

- Overactive thoughts

- Painful sex

- Rapid pulse

- Sensitive/amplified hearing

- Sexual (nondescript)

- Shortness of breath/breathing problems

- Sore breasts

- Spasms/twitching/tremors

- Stroke/heart failure in elderly

- Suicidal thinking

- Suicide attempts

- Swollen/tight/cramped

- Tired

- Tired, but cannot sleep

- Trouble/Inability to achieve orgasm

- Urge to urinate/ wet myself

- Urinary hesitance

- Weakness

- Weight gain

- Weight loss

- Worsened / induced mania

- Worsened anxiety

- Worsened drug cravings

- Zombie
- Panic attacks

- Sex drive

- Sleep, at high doses 
- Anger/irritability/rage/impulse control

- Internal thoughts/reactions

- My chemical imbalance

$\circ$ No Initial Benefits

○ No Initial Side Effects

○ No Side Effects

○ Timing

- Initial effect

- Newly emerged effect

- Over time

- FINANCES / THE SYSTEM

- Jumping through hoops

- FRIENDS AND FAMILY

- Disconnect between family perception and user experience

- Dosing/tapering as they did

- Drug worked well for them

- MY DIAGNOSIS / REASON FOR USE

- Anger/Irritability/Impulse Control

- Anti-psychotic

- Anti-psychotic, at higher doses

- Anxiety

- Bipolar

- Depressed / Depression

- Fear / Inability to live life

- For a diagnosis

- For an effect

- For sleep

- I am NOT -blank-
- I feel out of control / cannot shut mind off

- Insomnia due to...

- Manic/manic-depression

- Migraines

- OCD

- Schizophrenia / Schizoaffective

- Situational depression

- Stressful job

- Unable to function due to tragedy
○ I asked for the drug

- Report to / Listen to your doctor

- Seems extreme

- Talk to doc / How to talk to doc

- Unsure how much I trust my doctor

- Verifying Doc's info / Is my pdoc right?

\section{- MY DOCTOR}

- Changing Doctors

- New docs always change your meds

- Doctor doesn't believe / listen to me

- Doctor identified side effect

- Doctor suggested / Doctor put me on

- Increase/decrease/adjust dose

- Liquid form of drug

- Lubrication/toys for sex

- Ride out the storm 
- Doctor was wrong about effects

○ Doctor wouldn't help me wean off

- GP or Pdoc

○ I am not a doctor, but...
- Side effect trade-off

- Switch meds

- Taper SLOWLY / Low starting dose

- Vitamins and supplements

\section{- MY DRUG}

\section{○ Contra-indications}

$\bigcirc$ Dealing with Side Effects / Finding Optimum Effectiveness

- Add another med

- Avoid/cut down on caffeine/ alcohol

- Changing time of day

- Diet/nutrition

- Differs by time of day

- Drink more coffee/caffeine

- Eat a snack with drug

- Eventually goes away

- Exercise/stay physically active
- My usual state / I know my body

- No family history

- Stop/restart drug

- While on med

- Increased dose, no help/harm

- Take exactly as prescribed

\section{- Drug Action}

- Regulates

- Targets/restores brain molecules/chemicals

○ Drug-Drug Interactions

- Don't take this combination

- Possible effects of combo

- Try this combo

○ Evidence of Causality

- Anecdotal

- Drug or other drug?

- Drug or pre-existing condition?

- Medical tests

o Finding the Right Dose

- Adjusted/tapered myself

- Different effects/uses at different doses

$\bigcirc$ Judging Effectiveness

- OK, but not great

- YMMV

- Returned to this drug once again

- So many damn meds for so many years

- This time seems different! / The best yet!

- Now I need to work on my...

- Poop out

- Worsens other conditions

- No better choices

- Not sure

- Side effects are too great 
- Rating scale

○ My Drug Experience

- A miracle/saved my life

- I hate this drug

- It's not like what people say it is

- Search for the Right One

- Doing my research

- Going back to another drug

- It's starting to kick in

- Not enough time / too soon to tell

- Other drugs made it worse

○ Unsolved or Additional Problems

- Helped with one, but not the other

- Helps only for a few days

- More meds for other problems

○ Weighing Benefits vs Risks/Side Effects

- All in all, I am positive / Side effects tolerable

- Benefits not enough

- Feel great, no side effects

- PHARMACEUTICAL COMPANIES

O Brainwashed doctors

- FDA approval status

- It's a business

o PI Sheet

- Three percent my ass!

$\circ$ Sex is only a problem for med

- SUPPORT AND ADVICE

○ A good mindset

○ Drug Recommendations

- Better alternative than benzos

- Doesn't help like benzos do

- Don't take this drug!

$\circ$ Seeking experiences / worried about effects 
O What to expect

○ Withdrawal

- Looking back on it...

- May cause dependence

- Wait and see

\section{APPENDIX D}

Most Recent Codebook: April 2010

\section{- CITATIONS AND LINKS}

○ Academic Journal / "Research shows..."

○ Government/University

○ Internet

○ PI Sheet

$\circ$ Within website

- DRUG EFFECTS

$\circ$ Classification of Effects

- Category of Effect

- "Side Effect"

- Best/Fun Effect

- Less serious/Less severe

- Serious/ Severe

- Worst/ Most Annoying Effect

- Frequency

- Frequent

- Infrequent

- Rare

- Timing

- Initial Effect

- Newly Emerged/ Over Time

○ Contraindications (Only for Lex \& Ser)

- For certain people/age groups

- With alcohol or illicit drugs

- With certain foods

- With other medicines

○ Discontinuing / Missed Doses (Only for Lex \& Ser)

- Gastrointestinal disc effects 
- Neurological disc effects

- None / No withdrawals

- Other disc effects

- Psychiatric/emotional/ behavioral disc effects

$\circ$ Effects (Only for Lex \& Ser)

- Appetite and Weight Effects

- Increase appetite/ cravings

- Loss of appetite

- Weight gain

- Weight loss

- Gastrointestinal and Urinary Effects

- Constipation

- Diarrhea/Gas

- Menstruation Irregular

- Nausea/ sick stomach/vomiting

- Urinary changes/ problems

- Head or Face Effects

- Headache/Migraines Reduced

- Headache Increased

- Hearing, taste, smell changes

- Jaw clenching/grinding

- Vision/eye changes

- Lab Tests and Chronic Conditions

- Blood pressure changes

- Blood related changes

- Blood sugar high / Diabetes

- Cholesterol high

- Liver levels/Hepatoxicity/Hepatitis

- Other labs or chronic conditions

- Mental or Mood Effects

- Ability to do things in absence of symptoms

- Agitated / Restless

- Brain fog / Zombie

- Crying spells / Emotional rollercoaster

- Dreams vivid / nightmares

- Emotionally stable / calm / "normal"

- Energy increased / Euphoria / Mania

- Memory loss

- Numb/ detached / lack of energy or interest

- Other mental/mood changes

- Suicidal thinking/planning/attempts

- Symptoms new/worsened

- Symptoms reduced/improved

- Musculoskeletal and Neurological Effects 
- Abnormal movements

- Dizzy/Lightheaded/Faint

- Heaviness/Soreness/Pain/Numbness

- Neuroleptic Malignant Syndrome

- Seizure

- Swollen/Tight/Cramped

- Weakness

- Zaps/Tingling

- Nose, Throat, Chest Effects

- Breast soreness/lactation

- Breathing problems/Shortness of breath

- Chest pain/ heartburn

- Difficulty swallowing / speaking

- Dry mouth / sore throat

- Fever/ Chills / Infection

- Heart Disease/ Inflammation

- Heartbeat changes

- Nasal changes

- Other Effects

- Allergic reaction

- Alleviates side effect from another drug

- Hot flashes / heat stroke

- Hungover / Drunk

- No/Limited side effects

- Other misc effects

- Sexual Effects

- Increased sex drive

- Lost sex drive/libido

- Other sexual effects

- Trouble achieving orgasm

- Skin Effects

- Bruising

- Hair loss/thinning

- Itching/Rash

- Stevens-Johnson Syndrome

- Sweating like a pig

- Sleep Effects

- Difficult to wake up

- Extreme sleepiness / Tired

- Helps me sleep

- Trouble sleeping / Insomnia

- Yawning

\section{- FINANCES / THE SYSTEM}

$\circ$ Expense / Insurance

○ Jumping through hoops / Hospitals 


\section{- FRIENDS AND FAMILY}

$\circ$ Drug did *this* to them / Doing what they did

$\circ$ Family/friend observed an effect

$\circ$ Other family/friend issues

\section{- MY DIAGNOSIS / REASON FOR USE}

○ Anger / Irritability / Impulse Control

o Anxiety / Panic

- Bipolar / Mania

$\circ$ Change of life

○ Depressed / Depression

- Eating problems

- For sleep/insomnia

o I am -NOT- blank

- Inability to function/ Stressful life situation

o Migraines

$\circ$ Other reason / diagnosis

- PTSD

- Schizophrenia / Psychosis / Anti-Psychotic

- Skeptical of my diagnosis / Misdiagnosed

\section{- MY DOCTOR}

- Doctor won't listen / We disagree / I wasn't informed

$\circ$ GP or Pdoc?

○ I'm taking control / I'm my own best advocate

o Other doctor-related issues

- Talking with doc / Doc explained / Consult doc

$\circ$ Trusting the doctor?

\section{- MY DRUG}

○ Assessing the Overall Experience

- A miracle / saved my life / I recommend (Lex/Ser only)

- All in all, I am positive / Side effects tolerable

- I hate this drug / Stay away from it (Lex/Ser only)

- No or limited helpfulness / It's not my wonder drug

- Not worth it / Side effects too great

- YMMV

○ Dealing with Side Effects / Finding Optimum Effectiveness

- Add another med

- Avoid / Drink more caffeine/alcohol

- Changing time of day

- Check blood levels

- Diet / Food / Exercise

- Differs by time of day

- Dose adjustment

- Learn to live with it 
- Other methods

- Ride out the storm / Eventually goes away / Waiting

- Routine

- Switch meds / Stop med

- Taper SLOWLY / Low starting dose

- Vitamins and supplements

○ Do I Need Meds

- Drugs won't work / I'm done / I don't need it

- Going back on meds / Meds may be the solution

- Looking back on it.../ Since I stopped the med...

- Making a plan / Just in case...

- Trying/Tried alternative treatment

○ Evidence

- Anecdotal

- Drug or Something Else?

- Efficacy/Safety not established

- Medical tests

- My usual state / I know my body

- Stop/Restart drug

o Other Drug Issues

- Dependence / Withdrawal

- Diagnoses / Symptoms

- Dose related issues / advice

- Generic/other drug equivalence

- OTHER

- Poop out / Effects changed

○ Other Drugs and Drug Combinations

- Don't take this drug/combination

- My drug combo / so many damn meds...

- Possible effects / What to expect from other drugs

- Try this drug/combination

○ Theories of Drug Action

- Targets/Restores brain molecules/chemicals/neurotransmitters

- Unsure about mechanism/Other

- PHARMACEUTICAL COMPANIES / FDA

○ FDA approval status

O It's a business

○ Other pharma/regulatory/FDA issues

○ Three percent my ass!

- SUPPORT AND ADVICE

○ Other general support

○ Seeking experiences / worried about effects 


\section{APPENDIX E}

Most Recent Codebook with Descriptions of Codes

\section{CITATIONS AND LINKS}

- Academic journal/ "Research shows...": Cites, quotes summarizes, references, or links to an academic or medical journal, or to a published book or text; Also references such as "clinical trials show..." or "research shows..."

- Government/University: Cites, links, quotes, summarizes information from a government website or University's website

- Internet: Cites, links, quotes, summarizes information from an Internet website that is non-academic, non-governmental; Examples may be medicalnewstoday.com or Wikipedia.com

- PI Sheet: Cites, quotes, summarizes, references, or links to drug label or patient information sheet that accompanies the drug

- Within website: Cites, quotes, summarizes, references, or links to information within the same website; Examples include a hyperlink on WebMD that links to another page on WebMD, or a hyperlink on a CrazyMeds discussion that links to another page on CrazyMeds

\section{DRUG EFFECTS}

- Classification of Effects

\section{o Category of Effect}

- "Side Effect": Effects is listed or identified as a "side effect"

- Best/Fun Effect: Effect is described as the best effect, "my favorite effect," or similar; Effect is described as fun, enjoyable, cool.

- Less serious/Less severe: Effect is described as less serious, less severe, "not too bad," mild, "just a little," or similar 
- Serious/Severe: Effect is described as serious, severe, strong,

"really bad," extreme, or similar; Or effect results in an emergency room visit

- Worst/Most annoying effect: Effect is described as the worst effect, the worst side effect, "my least favorite effect," annoying, frustrating, or similar

o Frequency

- Frequent: Common, typical, or frequent effect

- Infrequent: Infrequent, not common effect

- Rare: Uncommon, rare effect

o Timing

- Initial Effect: Described as initial or immediate effect(s) of the drug; Effect appeared immediately or almost immediately upon taking the drug

- Newly emerged/Over time: Effect is a new effect that began after having been on the drug for some time, such as "this drug has always worked great, but after 2 years on it I am starting to sleep 16 hours a day" or "as I continued to take the drug, my anxiety levels increased"; Effect is something that built up over time, such as "I gained 45 lbs over 9 months of using the drug"

- Contraindications

o For certain people/age groups: Drug is contraindicated for or should be used with caution for certain age groups (i.e., children, elderly) or certain people (i.e., pregnant women, breastfeeding women), or for people who have a certain medical history

o With alcohol or illicit drugs: Avoid alcohol and/or illicit substances while taking this medication

o With certain food: Dangerous interaction may occur if you take this drug with a certain food, i.e., grapefruit

○ With other medicines: Drugs may have a dangerous interaction with other drugs, such as anticholinergics, dopamine-like drugs, drugs that cause drowsiness, etc.

- Discontinuing/Missed Doses

o Gastrointestinal disc effects: Gastro-intestinal effects occur or may occur when discontinuing/withdrawing from the drug, or upon missing doses(s), including diarrhea, nausea, vomiting, etc.

o Neurological disc effects: Neurological effects occur or may occur upon discontinuing or withdrawing from the drug or upon missing dose(s), 
including spasms, headaches, zaps, movements, insomnia, fainting, dizziness, etc.

o None/No withdrawals: No noticeable effects upon discontinuing

o Other disc effects: All other effects that occur or may occur upon discontinuing or withdrawal of the drug or upon missed dose(s), such as "Some conditions may become worse when the drug is suddenly stopped"

o Psychiatric/emotional/behavioral disc effects: Tapering off the drug or discontinuation of the drug may cause or does cause psychiatric, emotional, or behavioral effects, including hallucinations, depression, irritability, mania, agitation, crying spells, feeling sick/crazy/abnormal, etc.

- Effects

○ Appetite and Weight Effects

- Increase appetite/cravings: Increased appetite, often experienced as a ravenous insatiable appetite, or making multiple trips to the refrigerator all through the night, and cravings for carbs and sweets; Also includes effect of "sleep eating," which is eating during sleep with no memory of it happening

- Loss of appetite: Reduced appetite, loss of appetite, no appetite

- Weight gain: Weight gain of any amount

- Weight loss: Weight loss of any amount

○ Gastrointestinal and Urinary Effects

- Constipation

- Diarrhea/Gas: Diarrhea, gas, and other changes in bowels

- Menstruation Irregular: Irregular menstruation, including missed periods, additional periods, heavy periods, painful periods

- Nausea/sick stomach/vomiting

- Urinary changes/problems: Changes or problems with urine, including changes in amount of urine, sudden urges to urinate, wetting oneself, urinary hesitance

o Head or Face Effects

- Headache/Migraines reduced: Reduced frequency or intensity of migraines or headaches

- Headache increased: Headache or migraine is induced, worsened, or increased by the drug

- Hearing, taste, smell changes: Changes in hearing (i.e., sensitive or amplified hearing), taste (i.e., bad, unpleasant, or odd taste in mouth), or smell

- Jaw clenching/grinding: Jaw clenching, teeth grinding 
- Vision/eye changes: Vision or eye change or problems, i.e., dilated pupils, odd eye movements, sore eyes, dry eyes, etc

$\circ$ Lab Tests and Chronic Conditions

- Blood pressure changes: Blood pressure high/increased or low/decreased

- Blood related changes: Blood related abnormal findings, including decrease in white blood cells, decrease in blood platelets, etc; Blood related changes, such as blood thinning, bleeding easily from nose, anus, internal organs, etc

- Blood sugar high/Diabetes: Increased or high blood sugar, at risk of diabetes, or diagnosis or diabetes

- Cholesterol high

- Liver levels/Hepatoxicity/Hepatitis: Abnormal liver levels, or hepatoxicity, or hepatitis, or other changes in liver functioning

- Other labs or chronic conditions: Other lab test results or development of chronic conditions, including pancreatitis, prolonged Q-T interval on EKG, thyroid abnormalities, and increased triglycerides

o Mental or Mood Effects

- Ability to do things in absence of symptoms: Ability to do things (i.e. drive, work, go outside) that a person may have previously unable to do because of anxious feelings, depression, etc, or better performance or functioning at work, school, etc.

- Agitated/Restless: Agitation, feeling as though they want to jump out of their skin, or crawl out of their skin; jitteriness, spazzy, restless, can't sit still, akathisia, etc.

- Brain fog/Zombie: Cognitive slowing, including problems speaking, slurred speech, stuttering, unable to articulate words or thoughts, slowed problem-solving abilities; Difficult to focus or concentrate, confusion, mind fuzziness, foggy head, cloudy

- Crying spells/Emotional rollercoaster

- Dreams vivid/nightmares: Vivid dreams; odd, bizarre, or crazy dreams that may include nightmares or may include fun/pleasant dreams

- Emotionally stable/calm/"normal": Emotions and/or outward reactions are stable/steady, and not explosive or out of proportion to the situation; Emotions are appropriate to the situation; Can handle stress, problems, difficult situations; Feeling calm, relaxed, or at ease with life; More comfortable with one's life; Less 
despair, less hopeless; Also for comments such as "I felt more normal," "I felt like myself again," etc

- Energy increased/Euphoria/Mania: Increased energy, or high energy, which could be perceived as desirable or undesirable; Giddiness, excessive cheerfulness, "wired," "talking a mile a minute," euphoria, feeling "high," feels like taking speed or cocaine; Also includes induced or worsened hypomania, mania

- Memory loss: Memory loss, including sleep-walking type events

- Numb/Detached/Lack of energy or interest: Feeling of no emotions (not happy, not sad, not depressed, not anything_; flat emotion, flat feeling of nothingness; Lack of energy, lack of motivation, no interest in life, apathy; Feeling numb or detached from emotions, from life, from the world; feeling disassociated or unreal

- Other mental/mood changes: Other mental or mood changes not specified by a code in this category

- Suicidal thinking/planning/attempts: Thoughts of suicide or actively planning suicide; suicide attempts, including acts of selfinjury/self-harm

- Symptoms new/worsened: New, worsened, or increased symptoms, including anger, aggression, being "mean," irritable, anxiety, fear, panic, impulsive, depressed, hallucinations, psychosis, overactive/bizarre /uncontrollable thoughts or thinking, paranoia

- Symptoms reduced/improved: Reduction in or alleviation of symptoms, including anger, rage, aggression, "meanness," depression (i.e., depression lifted, smiled and laugh more, happier), anxiety, fear, panic, hallucinations, mania, clearer thoughts, fewer negative thoughts

o Musculoskeletal and Neurological Effects

- Abnormal movements: Abnormal movements, including tardive dyskinesia, or effects identified as possible or definite dyskinesia, Parkinson symptoms, and spasms, twitching, shakes, tremors, jerks, restless legs, etc

- Dizzy/Lightheaded/Faint

- Heaviness/Soreness/Pain/Numbness: Heaviness, soreness, pain, or numbness in legs, feet, arms, hands, or joints

- Neuroleptic Malignant Syndrome

- Seizure

- Swollen/Tight/Cramped: Swollen or tight arms, cramping in extremities or muscles 
- Weakness: Weakness in muscles or extremities

- Zaps/Tingling

o Nose, Throat, Chest Effects

- Breast soreness/lactation: Breasts swollen or sore, or lactation/discharge of milk from breasts

- Breathing problems/Shortness of breath

- Chest pain/heartburn

- Difficulty swallowing/speaking

- Dry mouth/sore throat: Dry mouth or throat; Sore or irritated throat

- Fever/Chills/Infection: Fever, chills, other flu-like symptoms, or signs of infection

- Heart disease/Inflammation: Heart disease, inflammation of the muscle of the heart, or other heart-related conditions

- Heartbeat changes: Heart throbbing or pounding, and/or rapid heartbeat or pulse, palpitations, slowed heartbeat, etc.

- Nasal changes: Nasal congestion, snoring, nose bleeds, runny nose, etc

$\circ$ Other Effects

- Allergic reaction

- Alleviates side effect from another drug: Seroquel or Lexapro relieves, improves, or alleviates an effect caused by another medication the person is taking

- Hot flashes/heat stroke: Ho flashes, heat stroke, or increased risk of heat stroke in hot weather

- Hungover/Drunk

- No/Limited side effects: Drug has no side effects, or virtually no side effects; Side effects are limited, or person specifically states the drug sis not have a certain effect, i.e., "I've had not sexual side effects"

- Other Misc Effects: Other misc effects not otherwise categorized o Sexual Effects

- Increased sex drive

- Lost sex drive/libido: Decrease in sex drive, no interest in sex, loss of libido

- Other sexual effects: Other sexual effects that do no fir the other codes, i.e., a nondescript remark about "sexual side effects," painful sex, prolonged erection, or premature ejaculation 
- Trouble achieving orgasm: Difficult to achieve orgasm, difficult to obtain an erection, difficult to achieve lubrication, lack of sensation

o Skin Effects

- Bruising

- Hair loss/thinning

- Itching/Rash

- Stevens-Johnson Syndrome

- Sweating like a pig: From increased perspiration to profuse sweating

\section{o Sleep Effects}

- Difficult to wake up: Difficult to wake up in the morning, which may include comments about sleeping through alarm clocks, unable to get out of bed until noon, etc.

- Extreme sleepiness/Tired: From drowsiness to extreme fatigue, as well as excessive sleeping or napping, often referred to as "extreme sleeping" or "extreme sleepiness"

- Helps me sleep: Drug effect is improved sleep; helps person sleep better

- Trouble sleeping/Insomnia: Trouble sleeping, unable to sleep, unable to fall asleep, not sleeping soundly, tired but cannot sleep; includes worsened insomnia

\section{FINANCES / THE SYSTEM}

- Expense/Insurance: Any comment related to the expense of the drug, i.e., the drug is too expensive or unaffordable; the drug is affordable; Any comment related to insurance issues, i.e., insurance was cancelled so the person can't afford the drug

- Jumping through hoops / Hospitals: Description of the obstacles to navigating "the system," i.e., trying to find another doctor, waiting lists and referral systems, too many patients and too few doctors, having to find a new doc, having to get a new doc because of insurance, etc; Description of or feelings about hospital experiences (positive or negative) and issues around involuntary hospitalization and forced medications

\section{FRIENDS AND FAMILY}

- Drug did *this* to them / Doing what they did: Comment regarding how a drug worked or did not work for a friend or family member, i.e., friend or 
family member did well on a specific drug and that influenced the User's choice of drug or it influenced their evaluation of their own drug experience, i.e., "I think I'm just a quirk because most people I know were just fine" on the drug; Any other anecdotal story about a person(s) who have had a particular experience with the drug; Following the experience of a friend or family member, i.e., "I am tapering off this drug the same way my sister did"

- Family/friend observed an effect: An effect of the drug is pointed out, observed, noticed by a family member or friend

- Other family/friend issues: Any other comment or information related to family or friends, such as how their family is supportive or not supportive of their treatment decisions, or how they get along with, take care of, or fight with their family

\section{MY DIAGNOSIS/REASON FOR USE}

- Anger / Irritability / Impulse Control

- Anxiety / Panic: Anxiety or Anxiety Disorder, including all anxiety disorders in the DSM (generalized anxiety disorder, social anxiety disorder, panic disorder, etc)

- Bipolar / Mania: Bipolar or Bipolar Disorder; Mania, Manic-Depression

- Change of life: "Change of life” signs; Hot flashes

- Depressed/Depression

- Eating problems: Eating-related problems, including anorexia, compulsive eating

- For sleep / Insomnia: Drug is taken to help with sleep or insomnia, including insomnia due to another problems (i.e., insomnia due to anxiety)

- I am -NOT- blank: Reason for use is partly defined by the diagnosis that a person DOES NOT have. This may include hesitance by a person to use the drug because they do not have a particular diagnosis, i.e., "I was very freaked out for the fact that I am NOT bipolar"

- Inability to function / Stressful life situation: Drug is used for a person who feels out of control in their lives, they cannot "shut my mind off" or calm down, unable to function due to fear or overactive thoughts; Person is under too much stress and cannot handle problems/life; Drug sis used to help a stressful life situation, i.e., anxiety due to a stressful job, depression due to family problems, a tragedy or after having a child, etc

- Migraines 
- Other reason/diagnosis: Using the medication for a reason or diagnosis not listed, i.e., as a preventative, to counteract side effects of another medication, to help during withdrawal from another medication, for ADHD, fibromyalgia, IBS, etc

- PTSD

- Schizophrenia/Psychosis/Anti-Psychotic: Schizophrenia, Schizoaffective, Psychotic Disorder, Psychosis, used as an antipsychotic

- Skeptical of my diagnosis/Misdiagnosed: There is doubt, skepticism, or uncertainty around their diagnosis; Person is misdiagnosed, or has been misdiagnosed, and perhaps was put on the "wrong" drug because of the misdiagnosis, i.e., "I am bipolar, but was misdiagnosed depressed and put on Lexapro"

\section{MY DOCTOR}

- Doctor won't listen / We disagree / I wasn't informed: Any general disagreement of conflict between the patient and doctor, i.e., "My doctor thinks this is a miracle pill for long-term management of my symptoms, but I am ready to get off this drug;" Doctor is wrong about an effect, i.e., "my pdoc said this would help my insomnia, but it made it worse;" Doctor discounts, minimizes, or invalidates an effect the user reports, and/or the User states that their doctor does not listen to them or believe them about some other medication-related issue, i.e., their doctor doesn't heed their caution about using a drug they had a bad experience with in the past; Doctor will not help the patient wean off the medication or does not support the patient in wanting to switch medications; The person states they feel like they were misinformed or not informed well about the drug or drug effects.

- GP or Pdoc ?: Any comment regarding what type of practitioner to use for medication-related decisions and prescriptions, i.e., a general practitioner versus a psychiatrist versus a neurologist versus a pharmacist, etc

- I'm taking control/I'm my own best advocate: Person does their own research, monitors their own treatment or condition, or makes some treatment-related decision on their own, for example by quitting the drug on their own, asking their doctor for a specific drug, etc; Comments about having to get informed in order to advocate for yourself to the doctor and others

- Other doctor-related issues: Any other comment or issues related to the doctor, such as advice about looking for a new doctor, comments about 
how supportive the doctor is, or other comments that do not fir in the other codes

- Talking with doc / Doc explained / Consult doc: Recommendation to consult, discuss with, or report to your doctor, and/or to listen to your doctor's instructions; Advice or information about how to talk to your doctor or how to prepare for a doctor's visit; Include any other doctormade decision, i.e., my new pdoc decided to change up all my meds, or include anything explained by the doctor, i.e., my doctor explained that my jitteriness might be a side effect of the drug

- Trusting the doctor?: Involves all issues of trust in relation to the doctor; User describes how they are unsure if or how much they trust their doctor or doctors in general; User describes some information their doctor gave them and they want to verify the information with other users; User describes the doctor's decision, recommendation, or lack of attention to a problem as seeming unusual, strange, or extreme

\section{MY DRUG}

- Assessing the Overall Experience

- A miracle / saved my life / I recommend: The drug or a drug effect is described as a miracle or as having saved their life; may also include language like the drug was wonderful, amazing, the best one yet, changed my life, etc; Also code if the drug is recommended because the person loved it or had a great experience with it

o All in all, I am positive / Side effects tolerable: Benefits of the drug outweigh side effects, risks, and/or costs; There may still be some side effects, but, all in all, the user feels positive about the drug; Side effects/adverse effects/costs (including financial costs) are insignificant and/or tolerable compared to the benefits of the drug

o I hate this drug/Stay away from it: I hate this drug, do not like this drug, would not take this drug again, had a bad experience on this drug; Recommendation to stay away from the drug or not to take the drug

○ No or limited helpfulness/It's not my wonder drug: No beneficial effects, few or limited beneficial effects; Drug is okay but not great; It's no miracle drug; Any comment regarding only limited helpfulness of the drug; The drug only partially helps solve a problem, or helps one problem but not other problems, i.e., Helped my anxiety, but not my depression; Also includes comments about 
having to weigh pros and cons of a drug, but without having reached a decision in the comment itself (otherwise, it would be coded 'all in all, I am positive' or 'side effects too great').

Weighing the pros and cons reflects the 'limited helpfulness' of the drug, i.e., it's no miracle cure

○ Not worth it / Side effects too great: Costs/side effects/adverse effects outweigh any benefits of the drug

- YMMV: Your Mileage May Vary; User may have a good or bad experience on the drug, but notes that other people may have a similar or different experience; Any acknowledgement that different people may have different experiences

- Dealing with side effects/Finding optimum effectiveness:

○ Add another med: Add a drug to Lex or Ser to help relieve or balance out certain drug effects, i.e., uses Wellbutrin with Lexapro to help their sex drive, or takes Ativan with Lexapro to help them sleep

- Avoid/Drink more caffeine/alcohol: Avoid or reduce caffeine and/or alcohol intake to reduce side effects or adverse effects, or to maximize drug effectiveness; Drink more caffeine (like coffee) or alcohol to reduce certain effects (like sleepiness) or maximize effectiveness

○ Changing time of day: Try different times of day to take the drug, in an attempt to increase effectiveness or reduce side effects

o Check blood levels: Monitor risk of diabetes and development of other conditions with regular tests, i.e., check blood sugar levels

$\circ$ Diet/Food/Exercise: Monitor diet or nutrition in order to reduce certain effects, like weight gain, or maximize effectiveness, including drinking lots of water, counting calories, staying away from carbohydrates, etc.; Take drug with food or drink, i.e., eat a snack with the drug to avoid nausea; Exercise or stay physically active to minimize side effects or maximize effectiveness

○ Differs by time of day: Side effects are better or worse depending on time of day, i.e., drowsiness in the morning goes away by evening

○ Dose adjustment: Counter, reduce, alleviate certain effects by adjusting the dose either up or down (usually down), and/or increase or decrease dose to increase effectiveness; Any comment that involves adjusting a dose, including splitting or crushing pills 
o Learn to live with it: Learn to live with, deal with, or just accept the drug; Comment about realizing that there is nothing to be done to relieve the effect, so the person must simply live with it

o Other methods: Any other strategy or method not already listed to help minimize side effects or maximize effectiveness, including stand up slowly to avoid dizziness, avoid heat, use lubrication for sex, report effect to a hotline (like poison control, FDA, or pharma company hotline), take PRN or as-needed by not every day, liquid form of drug, and "side effect trade-off"

o Ride out the storm / Eventually goes away / Waiting: Remark that "side effects" or "adverse effects" are temporary or will eventually go away with time; User describes being willing to ride out the side effects, assuming they will go away, or user describes how they kept with it through the side effects and found that they went away; Also, any comment that involves the concept of waiting, such as drug effects, particularly benefits, are not apparent immediately, but may "kick in" after a period of time of waiting; A person stops taking the drug and is waiting to see what happens next, for example, if withdrawal symptoms are going to be bad or if their depression will come back (a "wait and see" concept)

o Routine: Minimize side effects or maximize benefits by getting into a routine, i.e., a bedtime routine or a sleep routine

o Switch meds/Stop med: Recommendation or decision to switch meds and/or stop take the medication because a specific side effects has not gone away with time, and/or the drug has stopped working, did not work well, or had too many side effects

- Taper SLOWLY/Low starting dose: Recommend or advise to taper up or down very slowly, or to start with a low dose and taper up, or to not stop or quit a drug too quickly or "cold turkey"; Also code for any comment generally related to how to start, taper, or withdraw from a drug appropriately

○ Vitamins and Supplements: Using vitamins, supplements, and/or herbs to counteract or help relieve certain effects, i.e., Vitamins B5, B6, and magnesium, to help with fatigue

- Do I Need Meds?

o Drugs won't work/I'm done/I don't need it: Drugs are not the answer for a person's problems; Recommendation to a User that drugs may not be the answer to their problem, i.e., "Drugs aren't 
going to get you to sleep if your aversion is PTSD based. Only some heavy duty therapy is going to get around that; " user has had enough and is done with taking medications, i.e., "No more antidepressant!"; User wants to get off/to stop taking medications for whatever reasons, i.e., doesn't need them anymore, doesn't like them anymore, etc.

- Going back on meds/Meds may be the solution: Meds may be a long-term or lifetime solution, or the right solution for this person, or meds are definitely part of the overall whole situation; Decision to go back to taking meds, or a realization that meds are needed for them

o Looking back on it.../Since I stopped the med...: Comment regarding what has happened since the person has stopped taking the drug, a reflection of the drug experience now that the person is no longer on the drug, OR a reflection of the condition or problem now that the person is on meds. Some of these comments reflect positive changes (i.e., I feel so much better, more like myself now that I am off the meds; I never realized how depressed I was until I was put on Lexapro), or negative changes (i.e., now I see how much I really needed meds to stay stable; this med messed up my brain for years after having stopped taking it)

o Making a plan/Just in case..." Making a back-up plan, a prevention plan, a long-term plan, or a "just in case" or emergency plan

○ Trying/Tried alternative treatment: Tried or is trying an alternative treatment, such as talk therapy, CBT, DBT, ECT, EMDR, herbal remedies, etc in conjunction with or as an alternative to medication; Includes comments about medication not being the whole answer, but only part of a bigger plan that includes other treatment modalities or lifestyle changes for achieving or maintaining health

- Evidence

- Anecdotal: Description or explanation of an effect or a possible effect based on anecdotal evidence, i.e., from their observations, from their reading of discussion forums over the years, etc

○ Drug or Something Else?: An effect may come from the drug or from another source, including another drug, another condition (i.e., headaches from drug or from previous neck injury?), or from placebo effect; There is uncertainty as to the source of the effect, 
or it is explained why an effect comes from the drug versus another source

○ Efficacy/Safety not established: The efficacy and/or safety of the drug is not established, is uncertain, or unknown, i.e., efficacy of this drug has not been established for adolescents; Long-term effects are unknown; There is no evidence, a lack of evidence, or uncertainty in relation to a specific use/indication or effect, i.e., It is unknown if this drug passes into breastmilk

o Medical tests: Recommendation or description of medical tests (blood work, eye exams, for example) to help determine if an effect (i.e., fatigue, spasms, blurred vision) has a physical/biological basis

o My usual state / I know my body: Remark that a person (or "people" in general) know their own body enough to be able to tell if an effect is from a drug, i.e., User describes that usually they are one way, and now they are another way (usually their blood pressure is low, and now it is high); Also includes family history or lack of family history (of diabetes, for example) as evidence that the drug caused an effect

o Stop/Restart drug: Effect goes away once the person stops using the drug, and/or the effect stops once the person stops using the drug and returns once the user begins taking the drug again

- Other Drug Issues

o Dependence/Withdrawal: Any information or advice about dependence (i.e., caution that drug may cause dependence; drug did cause dependence for User), or withdrawal (i.e., how to withdraw or discontinue from the drug safely)

o Diagnoses/Symptoms: Information, advice, discussion about diagnosis or symptom-related issues, i.e., discussion about having depression vs bipolar vs drug-induced mania

o Dose related issues/advice: Any comment related to drug dosage; Information about maximum dosage, what dosage is too high, or drug overdose; any comment about taking the medicine exactly as prescribed, taking it consistently and regularly; Information on missed doses, i.e., what to do if a dose is missed; Also code for comments about the drug having a different effect and/or being used for a different reason at different doses, i.e., Seroquel is sleep inducing at low doses, and sleep neutral at high doses 
- Generic/other drug equivalence: Recommendation or description of a generic or other equivalents of the drug; may include dosing equivalents, i.e., "10mg celexa = 5mg or less of lexapro"; and/or comparison of similar drugs, i.e., contrasting effects of Lexapro and another SSRI

o OTHER: Garbage can code for any segments that do not fit elsewhere. Meds and pregnancy; meds and kids; I am new to meds so don't know what I should be feeling; street use or value of the drug' recycling or storage of meds; self-medicating with other substances

o Poop out/Effects changed: Drug stopped working after a period of time that it did work, or effects of the drug changed over time, i.e., "At first the drug helped keep me calm, but after taking it for a while I found myself getting angry and aggressive"

- Other Drugs and Drug Combinations

o Don't take this drug/combination: Recommend or advise to avoid a specific drug or combination; User identifies combination of drugs they used and states it was a poor combination or made things worse, didn't work for them, etc

- My drug combo/so many damn meds: Remark or description regarding a person's current drug combo, drug history, the various drugs and combinations of drugs the person has tried over the years, and past bad drug experiences; Note, if it is a recommendation or caution, please use other codes; General idea that they've tried everything, i.e., "I've taken every antidepressant (or whatever) on the market," tried other drugs that made their problems even worse, tried other drugs that had too many side effects, tried other drugs that were not effective; If a specific comparison of a previous drug and the current drug, then code under 'other drug equivalence'

o Possible effects/ What to expect from other drug: Description of possible effects to look out for or what a person can expect when taking a drug or combination of drugs, i.e., lowered seizure threshold from taking combo of antidepressant and multiple anticonvulsants, or "Lamictal is often activating, Abilify is..."; Code for effects or possible effects of any drug(s) other than lexapro or seroquel

- Try this drug/combination: List or identification of a drug or a combination of drugs that was or may be helpful for a person 
- Theories of Drug Action

○ Targets/restores brain molecules/chemicals/neurotransmitters: Drugs works by targeting or restoring chemicals in the brain; Includes comments about the drug being "potent," "selective," "targeting receptors, " etc

- Unsure about mechanism/Other: Not sure or not clear about the mechanism of action of the drug/how the drug "works;" Or any other theory of how the drug may work or have its effects, i.e., whether weight gain is caused by metabolic slowdown, stimulation of appetite, both, or something else; Other general references to "imbalance," "chemical imbalance," "messing with brain chemicals," etc.

\section{PHARMACEUTICAL COMPANIES / FDA}

- FDA approval status: Lists or clarifies FDA approval status for a drug and/or indication, including whether uses are approved or off-label/ unapproved

- It's a business: Remark that pharmaceutical companies are in the business of selling drugs; May include a description of a pharmaceutical company gimmick or way of doing business to maximize profits, i.e., launching a new drug when another one is about to go off patent; May includes doctors' role in prescribing medicines as a business. Or pharma's role in attempting to influence how doctors prescribe drugs $\mid$

- Other pharma/regulatory/FDA issues: Any other comment or information related to the pharmaceutical industry, regulatory industry or process, or other similar issues

- Three percent my ass!: Drug information from pharmaceutical manufacturers or on the drug label is limited, narrow in scope, is missing a particular effect, or somehow inaccurately estimates the scope of frequency of a drug effect, i.e., "according to pharma sexual side effects are only a problem for me"

\section{SUPPORT AND ADVICE}

- Other general support: Any comment giving general support or advice. Recommendation to keep a good/positive mindset when taking a drug. Comment that a person feels validated about their own experience after reading similar experiences of others on the website. Comment about the importance, helpfulness, strength of the "community" of online users

- Seeking experiences/worried about effects: User is wondering if others have experienced anything similar to what they have experience, and/or User is 
seeking others' experiences with a drug, which may be specific ("does this sdrug trigger hypomania?) or general ("what should I expect to happen when I take this drug?"); User describes feeling worried or scared about taking the drug, usually after having read about experiences of others on the websites, i.e., after reading about weight gain or other side effects

\section{APPENDIX F}

\section{Complete Log of Memo-Writing}

\begin{tabular}{|c|c|c|c|c|}
\hline Date & Case & Memo & Code & Consumer Comment \\
\hline $10 / 23 / 2009$ & $\begin{array}{l}\text { Case } \\
\# 27\end{array}$ & $\begin{array}{l}\text { We initially had two codes on } \\
\text { this segment: Side effects too } \\
\text { great and I hate this drug. I think } \\
\text { I'Il make a general coding rule to } \\
\text { use the All in all, I'm positive } \\
\text { code and Side effects too great } \\
\text { code if they can be applied } \\
\text { BEFORE the more general A } \\
\text { miracle code and I hate this drug } \\
\text { code. I want to avoid applying } 2 \\
\text { similar codes to the same } \\
\text { segment, and would prefer to } \\
\text { use the more specific code if it } \\
\text { fits before a more general code. }\end{array}$ & $\begin{array}{l}\text { Not worth it } \\
\text { / Side } \\
\text { effects too } \\
\text { great }\end{array}$ & $\begin{array}{l}\text { This lexapro works too much like a } \\
\text { neuroleptic..i hate feeling out of } \\
\text { control, detached... I would rather be } \\
\text { depressed than totally anihilated !!! }\end{array}$ \\
\hline 10/24/2009 & $\begin{array}{l}\text { Case } \\
\# 45\end{array}$ & $\begin{array}{l}\text { About being mis-informed or } \\
\text { uninformed about possible drug } \\
\text { effects?? Keep an eye to see if } \\
\text { this comes up more... } \\
12 / 1 / 09 \text { update: This came up a } \\
\text { couple times in the agreement } \\
\text { sample. I added this as a } \\
\text { dimension of Doctor was wrong - } \\
\text { - which is now "Doctor was } \\
\text { wrong / We disagree / I wasn't } \\
\text { informed" }\end{array}$ & $\begin{array}{l}\text { Doctor won't } \\
\text { listen / We } \\
\text { disagree / I } \\
\text { wasn't } \\
\text { informed }\end{array}$ & $\begin{array}{l}\text { Nobody warns you about the apathy } \\
\text { when you start this drug, had I known } \\
\text { that I would no longer care about the } \\
\text { people and things I love, I would have } \\
\text { never started taking this drug. }\end{array}$ \\
\hline
\end{tabular}




\begin{tabular}{|c|c|c|c|c|}
\hline Date & Case & Memo & Code & Consumer Comment \\
\hline 10/26/2009 & $\begin{array}{l}\text { Case } \\
\# 65\end{array}$ & $\begin{array}{l}\text { Emotionally stable/calm/ normal } \\
\text { and Brain fog/Zombie (and/or } \\
\text { Lack of interest code) may be } \\
\text { two sides of the same coin. It's a } \\
\text { mellowing effect that for some } \\
\text { people is just the "right amount" } \\
\text { of mellowing to the point of } \\
\text { feeling "normal" or able to } \\
\text { handle stressful situations, and } \\
\text { for other people is "too much" } \\
\text { mellowing to the point of } \\
\text { becoming a zombie or spacey } \\
\text { (or to the point of apathy). }\end{array}$ & $\begin{array}{l}\text { Emotionally } \\
\text { stable / } \\
\text { calm / } \\
\text { "normal" }\end{array}$ & $\begin{array}{l}\text { Noticed being very mellow the day } \\
\text { after the } 1 \text { st dose. The second day I } \\
\text { seemed a litte more myself, but at no } \\
\text { point felt "zombieish". }\end{array}$ \\
\hline 10/26/2009 & $\begin{array}{l}\text { Case } \\
\# 77\end{array}$ & $\begin{array}{l}\text { I am wondering how best to } \\
\text { characterize consumers' } \\
\text { categorization of effects. Right } \\
\text { now I have Best Effect and } \\
\text { Worst Effect, but am not sure } \\
\text { how representative that is. It's } \\
\text { not as straight forward as } \\
\text { experts' categorization of } \\
\text { "severe" "serious" "less serious" } \\
\text { etc. This particular case seems } \\
\text { to be an example of an aspect of } \\
\text { the drug that is cool or good, } \\
\text { maybe not the Best Effect, but } \\
\text { SOMETHING nonetheless. }\end{array}$ & $\begin{array}{l}\text { No / Limited } \\
\text { Side Effects }\end{array}$ & $\begin{array}{l}\text { Plus, you can have a few drinks on } \\
\text { them with no ill effects! SIDE } \\
\text { EFFECTS. Not many, }\end{array}$ \\
\hline $10 / 27 / 2009$ & $\begin{array}{l}\text { Case } \\
\# 125\end{array}$ & $\begin{array}{l}\text { I think I'll really want to do some } \\
\text { analysis on this code to reflect } \\
\text { all the dimensions of doctor told } \\
\text { me to do this, I'm afraid to ask } \\
\text { my doctor or mention this to my } \\
\text { doctor (as in the present } \\
\text { example), how should I bring } \\
\text { this up to my doctor, etc. This } \\
\text { seems like a rich category. }\end{array}$ & $\begin{array}{l}\text { Talking with } \\
\text { doc / Doc } \\
\text { explained / } \\
\text { Consult doc }\end{array}$ & $\begin{array}{l}\text { My question is: I'm seeing my doctor } \\
\text { next week and am planning to tell her } \\
\text { how well things are working out. Do I } \\
\text { mention these side effects? I'm afraid } \\
\text { she may want to switch me to another } \\
\text { med. While it's only been three weeks } \\
\text { - I really think the Celexa is working. }\end{array}$ \\
\hline $10 / 27 / 2009$ & $\begin{array}{l}\text { Case } \\
\# 129\end{array}$ & $\begin{array}{l}\text { Coded as "other methods" } \\
\text { because this person is agreeing } \\
\text { with the previous post, which } \\
\text { said: "I'm betting that the AD } \\
\text { makes you hypomanic/manic, } \\
\text { and you reach for a cig to calm } \\
\text { you down." }\end{array}$ & $\begin{array}{c}\text { Other } \\
\text { methods }\end{array}$ & $\begin{array}{l}\text { You hit the nail on the head. Come to } \\
\text { think of it, it explains what happened } \\
\text { before and what is happening now. }\end{array}$ \\
\hline
\end{tabular}




\begin{tabular}{|c|c|c|c|c|}
\hline Date & Case & Memo & Code & Consumer Comment \\
\hline $10 / 27 / 2009$ & $\begin{array}{l}\text { Case } \\
\# 133\end{array}$ & $\begin{array}{l}\text { Unsure if "stupid" and "dopey" } \\
\text { are more similar to feeling "high" } \\
\text { or feeling confused/fuzzy/foggy, } \\
\text { or something else. Come back } \\
\text { to this... } \\
\text { Update: For now I will combine } \\
\text { Zombie and Dopey and } \\
\text { determine later if that's } \\
\text { appropriate } \\
\text { Update 12/7/09: I combined all } \\
\text { of these together into Cogntive } \\
\text { Slowing / Foggy/ Dopey }\end{array}$ & $\begin{array}{l}\text { Brain fog / } \\
\text { Zombie }\end{array}$ & $\begin{array}{l}\text { Lexapro And Dopey Feeling Posted } \\
\text { by: Ginger Sun } 28 \text { September } 2008 \\
\text { 18:11:19 GMT +0000 I got a little } \\
\text { stupid on Lexapro, and it did } \\
\text { eventually go away. }\end{array}$ \\
\hline $10 / 27 / 2009$ & $\begin{array}{l}\text { Case } \\
\# 133\end{array}$ & $\begin{array}{l}\text { Person must just accept the side } \\
\text { effect, deal with it, live with it, } \\
\text { etc. }\end{array}$ & $\begin{array}{c}\text { Learn to live } \\
\text { with it }\end{array}$ & $\begin{array}{l}\text { I am afraid the weight is here to stay } \\
\text { until I get off the Lex. I am going to try } \\
\text { a little harder in my exercise, but I'm } \\
\text { not a hard-core workout nut like I was } \\
\text { in college, so I will probably end up } \\
\text { just maintaining where I am. }\end{array}$ \\
\hline $10 / 28 / 2009$ & $\begin{array}{l}\text { Case } \\
\# 143\end{array}$ & $\begin{array}{l}\text { This whole thread is about } \\
\text { people sharing their weight gain } \\
\text { stories on Lexapro. }\end{array}$ & Weight gain & $\begin{array}{l}\text { I guess I missed the part where } \\
\text { Lexapro causes weight gain I've been } \\
\text { steadily gaining weight the past four } \\
\text { years, but have only been on Lexapro } \\
\text { for about a year or so, maybe a year } \\
\text { and a half. }\end{array}$ \\
\hline $10 / 28 / 2009$ & $\begin{array}{l}\text { Case } \\
\# 147\end{array}$ & $\begin{array}{l}\text { Coded "Drug or Something } \\
\text { Else?" because previous poster } \\
\text { was suggesting the hair loss } \\
\text { was due to previous stress, } \\
\text { rather than the drug that the } \\
\text { person had only been on for } 4 \\
\text { weeks. The current post is } \\
\text { agreeing that that may be the } \\
\text { case. }\end{array}$ & $\begin{array}{l}\text { Drug or } \\
\text { Something } \\
\text { Else? }\end{array}$ & $\begin{array}{l}\text { May was my standout month for } \\
\text { extreme stress this year. Oh and } \\
\text { February. And all summer. No wonder } \\
\text { my hair is thin! }\end{array}$ \\
\hline $10 / 29 / 2009$ & $\begin{array}{l}\text { Case } \\
\# 173\end{array}$ & $\begin{array}{l}\text { Perhaps create a code about } \\
\text { other people - family, friends, } \\
\text { doctor, etc - observing an effect } \\
\text { the drug is having on the } \\
\text { consumer. } \\
12 / 1 / 09 \text { Update: I collapsed } \\
\text { some of the family codes into } \\
\text { one general "Other family/friend } \\
\text { issues" which inclues the } \\
\text { dimension above. }\end{array}$ & $\begin{array}{l}\text { Family/frien } \\
\text { d observed } \\
\text { an effect }\end{array}$ & $\begin{array}{l}\text { My partner has noticed that I seem } \\
\text { somewhat spaced out and forgetful } \\
\text { (minor things like car keys, turning on } \\
\text { dishwasher, not hearing what } \\
\text { someone is saying to me for a } \\
\text { moment, etc). }\end{array}$ \\
\hline
\end{tabular}




\begin{tabular}{|c|c|c|c|c|}
\hline Date & Case & Memo & Code & Consumer Comment \\
\hline 10/29/2009 & $\begin{array}{l}\text { Case } \\
\# 185\end{array}$ & $\begin{array}{l}\text { This comment in response to a } \\
\text { poster asking about what to } \\
\text { expect by taking her full dose of } \\
\text { Lexapro once a day rather than } \\
\text { split twice a day. Specifically } \\
\text { asking if there is a risk of mania. }\end{array}$ & $\begin{array}{l}\text { Dose } \\
\text { related } \\
\text { issues / } \\
\text { advice }\end{array}$ & $\begin{array}{l}\text { From Twice A Day To Once A Day } \\
\text { Posted by: Anna Thu } 4 \text { December } \\
200814: 28: 33 \text { GMT +0000 Anything's } \\
\text { possible when you are disturbing the } \\
\text { homeostasis... But honestly, I don't } \\
\text { think the risk is like, huge. Unless it } \\
\text { really hits your neurotransmitters like a } \\
\text { ton of bricks or something... }\end{array}$ \\
\hline $11 / 10 / 2009$ & $\begin{array}{l}\text { Case } \\
\# 96\end{array}$ & $\begin{array}{l}\text { I'm trying to distinguish "So } \\
\text { many damn meds" and "Other } \\
\text { drug equivalence." I am moving } \\
\text { comments like this one in which } \\
\text { there is a specific comparison of } \\
\text { or reference to a previous drug } \\
\text { and the current drug. More } \\
\text { general comments, like "I've } \\
\text { tried lots of meds..." will remain } \\
\text { under "so many damn meds." }\end{array}$ & $\begin{array}{l}\text { Generic/oth } \\
\text { er drug } \\
\text { equivalence }\end{array}$ & $\begin{array}{l}\text { I've been on lexapro for } 3 \text { years and } \\
\text { has been the only medication that has } \\
\text { really worked. I tried taking Zoloft and } \\
\text { it made my depression worse. }\end{array}$ \\
\hline $11 / 10 / 2009$ & $\begin{array}{l}\text { Case } \\
\# 239\end{array}$ & $\begin{array}{l}\text { Should this be coded as "Don't } \\
\text { take this combo" or as a } \\
\text { Contraindication? Maybe I need } \\
\text { to merge the two codes... } \\
12 / 1 / 09 \text { Update: This seems like } \\
\text { a clear contraindication now that } \\
\text { I read it again. But will need to } \\
\text { clearly distinguish or merge } \\
\text { these. }\end{array}$ & $\begin{array}{l}\text { With other } \\
\text { medicines }\end{array}$ & $\begin{array}{l}\text { By the way, if you start on SSRI } \\
\text { treatment, you should stop the } 5 \mathrm{HTP} \\
\text { since it may lead to } 5 \mathrm{HT} \text {-syndrome - } \\
\text { especially with Lexapro. }\end{array}$ \\
\hline $11 / 16 / 2009$ & $\begin{array}{l}\text { Case } \\
\# 123\end{array}$ & $\begin{array}{l}\text { Not sure whether to code No } \\
\text { beneficial effects or Ok, but not } \\
\text { great. I don't want } 2 \text { codes with } \\
\text { similar meaning for the same } \\
\text { segment. } \\
\text { 12/1/09 Update: This same } \\
\text { question had come up a few } \\
\text { times in the agreement coding. I } \\
\text { merged No beneficial effects into } \\
\text { Ok, but not great, as well as } \\
\text { pulled out the Limited } \\
\text { Helpfulness cases from Poop } \\
\text { Out and merged them into Ok, } \\
\text { but not great. }\end{array}$ & $\begin{array}{c}\text { No or } \\
\text { limited } \\
\text { helpfulness } \\
\text { / It's not my } \\
\text { wonder drug }\end{array}$ & $\begin{array}{l}\text { And I did not find that it was working } \\
\text { all that well to begin with, }\end{array}$ \\
\hline $11 / 27 / 2009$ & $\begin{array}{l}\text { Case } \\
\# 163\end{array}$ & $\begin{array}{l}\text { This comment is a response to } \\
\text { the previous post: "Lexapro left } \\
\text { me with my libido intact, but I } \\
\text { could not orgasm." }\end{array}$ & $\begin{array}{l}\text { Trouble } \\
\text { achieving } \\
\text { orgasm }\end{array}$ & $\begin{array}{l}\text { That totally happened to me, too! It } \\
\text { freaked me out and then I } \\
\text { remembered... Oh, this is a side-effect. } \\
\text { It was weird. }\end{array}$ \\
\hline
\end{tabular}




\begin{tabular}{|c|c|c|c|c|}
\hline Date & Case & Memo & Code & Consumer Comment \\
\hline $11 / 28 / 2009$ & $\begin{array}{l}\text { Case } \\
\# 185\end{array}$ & $\begin{array}{l}\text { Not sure whether to code } \\
\text { Dependence/Withdrawal, or } \\
\text { Taper SLOWLY. Again, do not } \\
\text { need } 2 \text { codes with similar } \\
\text { meanings on } 1 \text { segment. }\end{array}$ & $\begin{array}{l}\text { Dependenc } \\
\text { e / } \\
\text { Withdrawal }\end{array}$ & $\begin{array}{l}\text { since you are cross-tapering from one } \\
\text { ssri to another, there shouldn't be a } \\
\text { problem in going relatively quickly. The } \\
\text { slower taper that is recommended with } \\
\text { ssris is more about if you are coming } \\
\text { off completely, to avoid withdrawal } \\
\text { effects. }\end{array}$ \\
\hline $12 / 1 / 2009$ & $\begin{array}{l}\text { Case } \\
\# 115\end{array}$ & $\begin{array}{l}\text { We decided to use these codes } \\
\text { for consumer comments about } \\
\text { effects being "nothing intense," } \\
\text { "mild," "insignificant" and other } \\
\text { words like "less severe" or } \\
\text { "severe" }\end{array}$ & $\begin{array}{c}\text { Less } \\
\text { serious / } \\
\text { Less severe }\end{array}$ & $\begin{array}{l}\text { SIDE EFFECTS. Nothing intense, but } \\
\text { I have noticed that I'm always hungry }\end{array}$ \\
\hline $12 / 4 / 2009$ & $\begin{array}{l}\text { Case } \\
\# 60\end{array}$ & $\begin{array}{l}\text { This would fit under the code I } \\
\text { originally had under Support and } \\
\text { Advice: Feeling validated. This } \\
\text { refers to a person feeling like } \\
\text { their experience is validated } \\
\text { after reading about others' } \\
\text { experiences online. }\end{array}$ & $\begin{array}{l}\text { Other } \\
\text { general } \\
\text { support }\end{array}$ & $\begin{array}{l}\text { I spend most of my spare time in bed } \\
\text { sleeping - I'm barely participating in } \\
\text { life. I seriously thought I had chronic } \\
\text { fatigue syndrome, but after reading } \\
\text { about other people's experiences, now } \\
\text { I suspect it's the Lexapro. }\end{array}$ \\
\hline $12 / 4 / 2009$ & $\begin{array}{l}\text { Case } \\
\# 86\end{array}$ & $\begin{array}{l}\text { Sometimes the experience of } \\
\text { the drug is defined by what } \\
\text { effects are ABSENT. }\end{array}$ & $\begin{array}{l}\text { No / Limited } \\
\text { Side Effects }\end{array}$ & $\begin{array}{l}\text { Never had any tiredness or spaciness } \\
\text { on it. I also did not experience serious } \\
\text { sexual side effects. }\end{array}$ \\
\hline $12 / 4 / 2009$ & $\begin{array}{l}\text { Case } \\
\# 124\end{array}$ & $\begin{array}{l}\text { I'm wondering if I will need a } \\
\text { code like "Clinical trials show...". } \\
\text { Or if I should just put this under } \\
\text { the citation code? } \\
12 / 13 / 09 \text { Update: I've decided to } \\
\text { add this to the Citation code } \\
\text { "Academic Journal / Research } \\
\text { shows..." }\end{array}$ & $\begin{array}{l}\text { Academic } \\
\text { Journal / } \\
\text { "Research } \\
\text { shows..." }\end{array}$ & $\begin{array}{l}\text { He said that there are clinical trials that } \\
\text { show that } 80 \% \text { of those who will } \\
\text { respond to it at all will show some } \\
\text { change in the first } 2 \text { weeks }\end{array}$ \\
\hline $12 / 8 / 2009$ & $\begin{array}{l}\text { Case } \\
\# 130\end{array}$ & $\begin{array}{l}\text { In reference to worsened anxiety } \\
\text { upon starting Lexapro }\end{array}$ & $\begin{array}{l}\text { Symptoms } \\
\text { new / } \\
\text { worsened }\end{array}$ & $\begin{array}{l}\text { I just wanted to add that I was in the } \\
\text { same boat and I couldn't take ONE } \\
\text { day! You're awfully brave. }\end{array}$ \\
\hline $12 / 8 / 2009$ & $\begin{array}{l}\text { Case } \\
\# 133\end{array}$ & $\begin{array}{l}\text { This would fall into a code like: } \\
\text { "Accepting / Dealing with *it*" as } \\
\text { a method of dealing with (or not } \\
\text { being able to deal with?) side } \\
\text { effects. Rather the person is } \\
\text { having to learn to just live with it. }\end{array}$ & $\begin{array}{l}\text { Learn to live } \\
\text { with it }\end{array}$ & $\begin{array}{l}\text { Well, I have stayed in the vicinity of an } \\
\text { overall gain of } 8-10 \text { pounds. I did buy } \\
\text { some new pants. What else are you } \\
\text { going to do? I looked stupid in the } \\
\text { pants I had. I know I'll fit into them } \\
\text { when I get off this medication in } \\
\text { another } 6 \text { months, but I am just sort of } \\
\text { accepting it right now and dealing with } \\
\text { it. }\end{array}$ \\
\hline
\end{tabular}




\begin{tabular}{|c|c|c|c|c|}
\hline Date & Case & Memo & Code & Consumer Comment \\
\hline $12 / 8 / 2009$ & $\begin{array}{l}\text { Case } \\
\# 149\end{array}$ & $\begin{array}{l}\text { Another comment about } \\
\text { "accepting" or "dealing } \\
\text { with/learning to live with" side } \\
\text { effects. }\end{array}$ & $\begin{array}{c}\text { Learn to live } \\
\text { with it }\end{array}$ & $\begin{array}{l}\text { I'm only on here venting about how I } \\
\text { am stressed and concerned about } \\
\text { weight gain, not saying I have quit yet. } \\
\text { I need some time to accept the side } \\
\text { effects. }\end{array}$ \\
\hline $12 / 8 / 2009$ & $\begin{array}{l}\text { Case } \\
\# 156\end{array}$ & $\begin{array}{l}\text { I see this idea come up at times: } \\
\text { I don't plan on ever stopping this } \\
\text { drug / I will take this for a } \\
\text { lifetime, etc. I have been coding } \\
\text { these under "A miracle" and } \\
\text { possibly under "OTHER" at } \\
\text { times }\end{array}$ & $\begin{array}{l}\text { A miracle / } \\
\text { saved my } \\
\text { life / I } \\
\text { recommend } \\
\text { (Lex/Ser } \\
\text { only) }\end{array}$ & $\begin{array}{l}\text { I feel much less insane now, and don't } \\
\text { plan to stop taking it any time soon, I } \\
\text { haven't felt this serene in a long time. }\end{array}$ \\
\hline $12 / 9 / 2009$ & $\begin{array}{l}\text { Case } \\
\# 134\end{array}$ & $\begin{array}{l}\text { I highlighted this in addition to } \\
\text { Dependence/Withdrawal } \\
\text { because of the comment } \\
\text { referring to "Looking back on the } \\
\text { drug experience..." now they can } \\
\text { see that their creativity had been } \\
\text { zapped. } \\
12 / 13 / 09 \text { Update: Decided to } \\
\text { add a code called "Looking back } \\
\text { on it.../Since I stopped the } \\
\text { med..." because this concept } \\
\text { continues to come up. }\end{array}$ & $\begin{array}{l}\text { Looking } \\
\text { back on } \\
\text { it.../Since I } \\
\text { stopped the } \\
\text { med... }\end{array}$ & $\begin{array}{l}\text { I was having dinner and we had the } \\
\text { ketchup out, and Heinz has some } \\
\text { dumb commercial contest going on, } \\
\text { and I came up with a totally cool } \\
\text { commercial idea!! I wouldn't have been } \\
\text { able to think something like that up } \\
\text { when I was on the meds. But, at the } \\
\text { same time, I didn't notice that my } \\
\text { creativity was zapped while I was on } \\
\text { the meds. }\end{array}$ \\
\hline $12 / 9 / 2009$ & $\begin{array}{l}\text { Case } \\
\# 472\end{array}$ & $\begin{array}{l}\text { Added Best/Fun Effect code } \\
\text { here because I wanted to mark } \\
\text { her comment "a benefit I had not } \\
\text { anticipated", and that comment } \\
\text { does not really fit anywhere else. }\end{array}$ & $\begin{array}{c}\text { Best / Fun } \\
\text { Effect }\end{array}$ & $\begin{array}{l}\text { A benefit I had not anticipated was } \\
\text { while taking Lexapro I no longer had } \\
\text { sleep paralysis. }\end{array}$ \\
\hline $12 / 9 / 2009$ & $\begin{array}{l}\text { Case } \\
\# 472\end{array}$ & $\begin{array}{l}\text { She says it HELPED her hot } \\
\text { flashes, so I will have to break } \\
\text { the improves from the worsens } \\
\text { hot flashes during analysis. }\end{array}$ & $\begin{array}{l}\text { Hot flashes / } \\
\text { heat stroke }\end{array}$ & I also think it helped my hot flashes \\
\hline $12 / 9 / 2009$ & $\begin{array}{l}\text { Case } \\
\# 533\end{array}$ & $\begin{array}{l}\text { I did not code this My Drug } \\
\text { Combo (as I anticipate Kristina } \\
\text { will) because I thought Drug Or } \\
\text { Something Else? is more } \\
\text { specifically what this comment } \\
\text { refers to. }\end{array}$ & $\begin{array}{l}\text { Drug or } \\
\text { Something } \\
\text { Else? }\end{array}$ & $\begin{array}{l}\text { hard time getting up in the morning } . \text { I } \\
\text { am also on bp meds and klonopin } \\
\text { which all cause drowsiness. }\end{array}$ \\
\hline
\end{tabular}




\begin{tabular}{|c|c|c|c|c|}
\hline Date & Case & Memo & Code & Consumer Comment \\
\hline $12 / 9 / 2009$ & $\begin{array}{l}\text { Case } \\
\# 554\end{array}$ & $\begin{array}{l}\text { Ok, I am seeing that Taper } \\
\text { Slowly and } \\
\text { Dependence/Withdrawal are not } \\
\text { defined enough to clearly } \\
\text { differentiate them. I will have to } \\
\text { somehow merge them or } \\
\text { differentiate them (after we finish } \\
\text { agreement coding) }\end{array}$ & $\begin{array}{l}\text { Taper } \\
\text { SLOWLY / } \\
\text { Low Starting } \\
\text { Dose }\end{array}$ & $\begin{array}{l}\text { I will start tapering off of it after being } \\
\text { on it for a year. Take } 200 \mathrm{mg} \text { at } \\
\text { bedtime, now taking } 100 \mathrm{mg} \text { for a } \\
\text { month or two and going from there! }\end{array}$ \\
\hline $12 / 10 / 2009$ & $\begin{array}{l}\text { Case } \\
\# 573\end{array}$ & $\begin{array}{l}\text { After agreement coding, I think I } \\
\text { should merge Helps Me Sleep } \\
\text { with Tired/Excessive Sleeping - } \\
\text { and rename this new code } \\
\text { simply "Increased Sleep / } \\
\text { Sleepiness". As it stands now, } \\
\text { comments like this would be } \\
\text { difficult to classify as either } \\
\text { "Helps me" or "Excessive" }\end{array}$ & $\begin{array}{c}\text { Extreme } \\
\text { sleepiness / } \\
\text { Tired }\end{array}$ & I did sleep for 14 hours straight \\
\hline $12 / 10 / 2009$ & $\begin{array}{l}\text { Case } \\
\# 591\end{array}$ & $\begin{array}{l}\text { Not sure if this belongs here, or } \\
\text { a code under Muscoloskeletal } \\
\text { and Neurological Effects }\end{array}$ & $\begin{array}{l}\text { Brain fog / } \\
\text { Zombie }\end{array}$ & $\begin{array}{l}\text { slurred speech, problems with walking } \\
\text { correctly/balance. }\end{array}$ \\
\hline $12 / 10 / 2009$ & $\begin{array}{l}\text { Case } \\
\# 613\end{array}$ & $\begin{array}{l}\text { This would have fallen within } \\
\text { Side Effect Trade-Off, however I } \\
\text { deleted that code prior to this } \\
\text { last round of agreement coding } \\
\text { because I had not seen it come } \\
\text { up often. }\end{array}$ & $\begin{array}{l}\text { Other } \\
\text { methods }\end{array}$ & $\begin{array}{l}\text { Now at } 500 \mathrm{mg} \text { the difficulty falling } \\
\text { asleep has returned and I'm back to } \\
\text { waking up sweaty with bad dreams } \\
\text { more often. But my daytime sedation } \\
\text { has gone down. }\end{array}$ \\
\hline $12 / 10 / 2009$ & $\begin{array}{l}\text { Case } \\
\# 613\end{array}$ & $\begin{array}{l}\text { This is in response to a concern } \\
\text { about taking a } 300 \mathrm{mg} \text { dose - i.e., } \\
\text { whether that is too high, whether } \\
\text { they should start with something } \\
\text { lower, etc? }\end{array}$ & $\begin{array}{l}\text { Dose } \\
\text { related } \\
\text { issues / } \\
\text { advice }\end{array}$ & $\begin{array}{l}\text { Not just for the sake of being contrary, } \\
\text { I think the 'listen to your body' } \\
\text { approach can be appropriate in some } \\
\text { cases. NOTE: I am NOT saying it's ok } \\
\text { in ALL, or even MOST cases, just } \\
\text { SOME... especially with a med like } \\
\text { slurroquel where there's a huge dosing } \\
\text { range. }\end{array}$ \\
\hline $12 / 10 / 2009$ & $\begin{array}{l}\text { Case } \\
\# 613\end{array}$ & $\begin{array}{l}\text { I code these sorts of comments } \\
\text { with 'Waiting' because I interpret } \\
\text { this as the person is hoping } \\
\text { (read: waiting) to see what } \\
\text { happens next, i.e., whether the } \\
\text { weight will come off. }\end{array}$ & $\begin{array}{l}\text { Ride out the } \\
\text { storm / } \\
\text { Eventually } \\
\text { goes away / } \\
\text { Waiting }\end{array}$ & $\begin{array}{l}\text { I'm HOPING to experience rapid } \\
\text { weight loss as I taper down the } \\
\text { slurroquel. }\end{array}$ \\
\hline $12 / 10 / 2009$ & $\begin{array}{l}\text { Case } \\
\# 613\end{array}$ & $\begin{array}{l}\text { In response to a suggestion for } \\
\text { getting the "Anxiety and Phobia } \\
\text { Workbook" }\end{array}$ & $\begin{array}{l}\text { Trying/Tried } \\
\text { alternative } \\
\text { treatment }\end{array}$ & $\begin{array}{l}\text { I opted for Silver's suggestion of } \\
\text { Master your Panic, etc. Pretended that } \\
\text { I was ordering them for a client to } \\
\text { actually get me to get the books in the } \\
\text { house. Now would be a great time to } \\
\text { take them out! }\end{array}$ \\
\hline
\end{tabular}




\begin{tabular}{|c|c|c|c|c|}
\hline Date & Case & Memo & Code & Consumer Comment \\
\hline $12 / 11 / 2009$ & $\begin{array}{l}\text { Case } \\
\# 650\end{array}$ & $\begin{array}{l}\text { Nice example for illustrating how } \\
\text { the absence of an effect can be } \\
\text { as defining as the presence of } \\
\text { an effect. }\end{array}$ & $\begin{array}{l}\text { No / Limited } \\
\text { Side Effects }\end{array}$ & $\begin{array}{l}\text { I haven't gained any weight on it, } \\
\text { although that's more a matter of } \\
\text { avoiding a bad thing than saying a } \\
\text { good thing. }\end{array}$ \\
\hline $12 / 11 / 2009$ & $\begin{array}{l}\text { Case } \\
\# 675\end{array}$ & $\begin{array}{l}\text { This comment is in response to } \\
\text { a person asking if there is an } \\
\text { online Canadian pharmacy they } \\
\text { can get generic Seroquel? }\end{array}$ & $\begin{array}{l}\text { Generic/oth } \\
\text { er drug } \\
\text { equivalence }\end{array}$ & $\begin{array}{l}\text { Unfortunately we can't answer that } \\
\text { question. paypal are dicks about that } \\
\text { sort of thing, not that it's explicit in their } \\
\text { TOS or anything. Generic quetiapine } \\
\text { is available in other countries and if } \\
\text { you read } \\
\text { http://www.crazymeds.us//nternetPhar } \\
\text { macists.shtml you can find out on your } \\
\text { own. }\end{array}$ \\
\hline $12 / 11 / 2009$ & $\begin{array}{l}\text { Case } \\
\# 675\end{array}$ & $\begin{array}{l}\text { Not sure if I want to put these } \\
\text { comments about monitoring } \\
\text { blood levels for diabetes under } \\
\text { Dealing with Side Effects, or } \\
\text { under Medical Tests - Evidence } \\
\text { of Causality. It is a medical test, } \\
\text { but not having to do with } \\
\text { causality, but rather having to do } \\
\text { with Dealing with Side Effects. }\end{array}$ & $\begin{array}{l}\text { Learn to live } \\
\text { with it }\end{array}$ & $\begin{array}{l}\text { What's This About Seroquel And } \\
\text { Diabetes? Posted by: Jerod Poore Thu } \\
14 \text { August } 200812: 33: 47 \text { GMT }+0000 \\
\text { There's like this blood test you can get } \\
\text { from a doctor. Just being on Seroquel } \\
\text { with a family history of diabetes is } \\
\text { enough to warrant it. Viola! Question } \\
\text { answered. Get one every six months } \\
\text { and you're set. }\end{array}$ \\
\hline $12 / 11 / 2009$ & $\begin{array}{l}\text { Case } \\
\# 675\end{array}$ & $\begin{array}{l}\text { Quote is from female with } \\
\text { menstruation irregularities. }\end{array}$ & $\begin{array}{l}\text { Menstruatio } \\
\text { n Irregular }\end{array}$ & SEE QUOTE. It's damn rare, \\
\hline 12/11/2009 & $\begin{array}{l}\text { Case } \\
\# 675\end{array}$ & $\begin{array}{l}\text { Would code as "Learning to live } \\
\text { with it..." }\end{array}$ & $\begin{array}{l}\text { Learn to live } \\
\text { with it }\end{array}$ & $\begin{array}{l}\text { There's not much you can do about it. } \\
\text { It usually gets worse the longer you } \\
\text { take Seroquel. }\end{array}$ \\
\hline $12 / 11 / 2009$ & $\begin{array}{l}\text { Case } \\
\# 675\end{array}$ & $\begin{array}{l}\text { Talking about sensitivity to heat, } \\
\text { getting overheated, and terrible } \\
\text { sweats. }\end{array}$ & $\begin{array}{l}\text { Hot flashes / } \\
\text { heat stroke }\end{array}$ & $\begin{array}{l}\text { Am I The Only One Who Had This } \\
\text { Side Effect? Posted by: Jerod Poore } \\
\text { Mon } 31 \text { March } 2008 \text { 14:07:04 GMT } \\
+0000 \text { Hardly. And t he reason jook } \\
\text { didn't spot it is because AstraZenca } \\
\text { decided to use doctorese to describe } \\
\text { it. Asthenia. Fatigue, malaise, night } \\
\text { sweats, hot flashes, and a mimicking } \\
\text { of arthritis. Up to } 10 \% \text { of people who } \\
\text { take Seroquel reported this during the } \\
\text { clinical trials! }\end{array}$ \\
\hline $12 / 11 / 2009$ & $\begin{array}{l}\text { Case } \\
\# 747\end{array}$ & Code "Learning to live with it..." & $\begin{array}{l}\text { Learn to live } \\
\text { with it }\end{array}$ & $\begin{array}{l}\text { I've learned to live with this but I hope } \\
\text { no one else has to }\end{array}$ \\
\hline
\end{tabular}




\begin{tabular}{|c|c|c|c|c|}
\hline Date & Case & Memo & Code & Consumer Comment \\
\hline $12 / 11 / 2009$ & $\begin{array}{l}\text { Case } \\
\# 772\end{array}$ & $\begin{array}{l}\text { I should note that comments like } \\
\text { this have been coded under "so } \\
\text { many damn meds" and NOT } \\
\text { also under "limited or no } \\
\text { helpfulness." More specific } \\
\text { instances of a med not working } \\
\text { have been coded under the } \\
\text { latter. General references to } \\
\text { "none of the meds l've tried have } \\
\text { worked" or "I've tried } 4 \text { other } \\
\text { SSRI's and none of them worked } \\
\text { until I was prescribed Lexapro," } \\
\text { etc are coded under "so many } \\
\text { damn meds" }\end{array}$ & $\begin{array}{l}\text { My drug } \\
\text { combo / so } \\
\text { many damn } \\
\text { meds... }\end{array}$ & $\begin{array}{l}\text { I had tried everything and it didn't work } \\
\text { (ambien, anything herbal, ativan) and } \\
\text { seroquel works! }\end{array}$ \\
\hline $12 / 12 / 2009$ & $\begin{array}{l}\text { Case } \\
\# 974\end{array}$ & $\begin{array}{l}\text { I interpret the "they" in this } \\
\text { comment as referring to doctors. }\end{array}$ & $\begin{array}{l}\text { Other } \\
\text { doctor- } \\
\text { related } \\
\text { issues }\end{array}$ & $\begin{array}{l}\text { being on my meds- which they finally } \\
\text { got right after } 12 \text { years- allowed me to } \\
\text { accomplish things that I never would } \\
\text { have thought I was able to do. }\end{array}$ \\
\hline $12 / 13 / 2009$ & $\begin{array}{r}\text { Case } \\
\# 1003\end{array}$ & $\begin{array}{l}\text { Here again the dilemma of } \\
\text { having to choose between } \\
\text { mental and physical health }\end{array}$ & $\begin{array}{l}\text { No or } \\
\text { limited } \\
\text { helpfulness } \\
\text { / It's not my } \\
\text { wonder drug }\end{array}$ & $\begin{array}{l}\text { my gut is telling me to get off zyprexa } \\
\text { too, but i don't really know what my } \\
\text { alternatives are, and i do want to be } \\
\text { stabilized. it's a tough situation. }\end{array}$ \\
\hline $12 / 13 / 2009$ & $\begin{array}{r}\text { Case } \\
\# 1014\end{array}$ & Sleep eating & $\begin{array}{l}\text { Increase } \\
\text { appetite / } \\
\text { cravings }\end{array}$ & $\begin{array}{l}\text { I would eat in my sleep and even if I } \\
\text { was awake, I literally could not feel full, } \\
\text { no matter how much I ate. }\end{array}$ \\
\hline $12 / 17 / 2009$ & $\begin{array}{l}\text { Case } \\
\# 245\end{array}$ & $\begin{array}{l}\text { I highlighted because of the term } \\
\text { "adverse reactions" to describe } \\
\text { the effects. We've been coding } \\
\text { for "side effects", but not for } \\
\text { other terms that people may } \\
\text { use. Not sure if I need to return } \\
\text { to this and code them all under } \\
\text { "side effects" (to be sorted out } \\
\text { during analysis), add additional } \\
\text { terms for coding, or just ignore it } \\
\text { completely. }\end{array}$ & "Side Effect" & $\begin{array}{l}\text { I guess I'm having too many adverse } \\
\text { reactions. Let's see.....nausea, } \\
\text { migraine, upset stomach, trembling, } \\
\text { heart palpitations, dizzy, completely } \\
\text { lost appetite (can't eat at all). }\end{array}$ \\
\hline
\end{tabular}




\begin{tabular}{|c|c|c|c|c|}
\hline Date & Case & Memo & Code & Consumer Comment \\
\hline $12 / 17 / 2009$ & $\begin{array}{l}\text { Case } \\
\# 254\end{array}$ & $\begin{array}{l}\text { This person is definitely } \\
\text { weighing benefits vs costs, but } \\
\text { does not come to a decision. I } \\
\text { coded it as Side Effects Too } \\
\text { Great because she answers "no" } \\
\text { to her question about would she } \\
\text { have gone on Lexapro if she } \\
\text { knew about the weight gain. }\end{array}$ & $\begin{array}{l}\text { Not worth it } \\
\text { / Side } \\
\text { effects too } \\
\text { great }\end{array}$ & $\begin{array}{l}\text { great for panic attacks . bad for weight } \\
\text { Thanks to Lexapro, I no longer have } \\
\text { anxiety attacks. they are still there, but } \\
\text { they don't come full blast. . However } \\
\text { and this is a big HOWEVER, I have } \\
\text { gained 35lbs on this med. I can't lose it } \\
\text { no matter what... Had I known about } \\
\text { this side effect would have gone on } \\
\text { Lexapro? No... even though my life is } \\
\text { back... it's hard now because I am in a } \\
\text { size } 16 \text { and I am normally a size } 10 . . \\
\text { But which is better.. being anxiety free } \\
\text { and overweight? or skinny and panic } \\
\text { ridden? it's a hard question }\end{array}$ \\
\hline $12 / 30 / 2009$ & $\begin{array}{l}\text { Case } \\
\# 276\end{array}$ & $\begin{array}{l}\text { Relieved } \\
\text { withdrawal/discontinuation } \\
\text { symptoms caused by another } \\
\text { drug. }\end{array}$ & $\begin{array}{l}\text { Alleviates } \\
\text { side effect } \\
\text { from } \\
\text { another } \\
\text { drug }\end{array}$ & $\begin{array}{l}\text { Three days after stopping Zoloft I had } \\
\text { severe and constant dizziness, } \\
\text { increased blood pressure and heart } \\
\text { rate - so my DR thought I should try } \\
\text { going on something different as I } \\
\text { wasn't coping well going cold turkey. } \\
\text { Within an hour of starting Lexapro the } \\
\text { dizziness disappeared, my heart rate } \\
\text { dropped over } 24 \text { hours and I felt ok } \\
\text { again. }\end{array}$ \\
\hline $12 / 30 / 2009$ & $\begin{array}{l}\text { Case } \\
\# 278\end{array}$ & $\begin{array}{l}\text { Relieved side effects caused by } \\
\text { another drug. }\end{array}$ & $\begin{array}{l}\text { Alleviates } \\
\text { side effect } \\
\text { from } \\
\text { another } \\
\text { drug }\end{array}$ & $\begin{array}{l}\text { I tried lexapro to reduce the side } \\
\text { effects of effexor and hopefully have a } \\
\text { better mood elevation. It did solve the } \\
\text { sexual side effects }\end{array}$ \\
\hline $12 / 30 / 2009$ & $\begin{array}{l}\text { Case } \\
\# 280\end{array}$ & $\begin{array}{l}\text { Serotonin syndrome - I also } \\
\text { coded these effects individually. }\end{array}$ & $\begin{array}{l}\text { Other Misc } \\
\text { Effects }\end{array}$ & $\begin{array}{l}\text { After almost } 5 \text { weeks at a dose of } \\
10 \mathrm{mg} \text { I started to experience } \\
\text { symptoms of what seemed like, to me, } \\
\text { serotonin syndrome - agitation and } \\
\text { anxiety ; general tremor and tremor in } \\
\text { the jaw ; confusion ; headache ; } \\
\text { sweating etc. - so dropped my dose } \\
\text { down to } 5 \mathrm{mg} \text { and these effects } \\
\text { disappeared pretty quickly. }\end{array}$ \\
\hline $12 / 30 / 2009$ & $\begin{array}{l}\text { Case } \\
\# 283\end{array}$ & $\begin{array}{l}\text { Relieves side effects caused by } \\
\text { other drugs }\end{array}$ & $\begin{array}{l}\text { Alleviates } \\
\text { side effect } \\
\text { from } \\
\text { another } \\
\text { drug }\end{array}$ & $\begin{array}{l}\text { Have been on Lexapro two weeks, } \\
\text { best of Paxil, (gained weight/lost all } \\
\text { sex drive), Effexor, (severe headaches } \\
\text { and no sex drive), Wellbutrin, (gave } \\
\text { me a seizure second night), and now } \\
\text { Lexapro. So far better; sex drive } \\
\text { returning }\end{array}$ \\
\hline
\end{tabular}




\begin{tabular}{|c|c|c|c|c|}
\hline Date & Case & Memo & Code & Consumer Comment \\
\hline $12 / 30 / 2009$ & $\begin{array}{l}\text { Case } \\
\# 289\end{array}$ & $\begin{array}{l}\text { I am merging Flat feelings with } \\
\text { Feeling detached or unreal } \\
\text { because I think the emotional } \\
\text { numbness described here is a } \\
\text { central defining feature of each. } \\
\text { Feeling detached from their } \\
\text { emotions, from their lives, from } \\
\text { the world. }\end{array}$ & $\begin{array}{l}\text { Numb / } \\
\text { Detached / } \\
\text { Lack of } \\
\text { energy or } \\
\text { interest }\end{array}$ & $\begin{array}{l}\text { mainly consisted of a "detached" } \\
\text { feeling or what I would describe as } \\
\text { emotional numbness. }\end{array}$ \\
\hline $12 / 30 / 2009$ & $\begin{array}{l}\text { Case } \\
\# 296\end{array}$ & $\begin{array}{l}\text { Not really weight gain, but } \\
\text { "difficult to lose weight" - not } \\
\text { sure if I need an additional code } \\
\text { for this or just note it during } \\
\text { analysis. }\end{array}$ & Weight gain & $\begin{array}{l}\text { Lexapro seems to make it more } \\
\text { difficult to take weight off than before }\end{array}$ \\
\hline $12 / 30 / 2009$ & $\begin{array}{l}\text { Case } \\
\# 300\end{array}$ & Code: "Learn to live with it" & $\begin{array}{l}\text { Learn to live } \\
\text { with it }\end{array}$ & $\begin{array}{l}\text { I am able to squeeze in two sessions } \\
\text { of gym a week. I make a huge will- } \\
\text { power effort to control my apetite. But } \\
\text { I have also accepted that I am not } \\
\text { going to be back to my weight of three } \\
\text { years ago before Lexapro. And, } \\
\text { frankly, I am not adding Wellbutrin for } \\
\text { weight loss. It makes me jittery. }\end{array}$ \\
\hline $12 / 30 / 2009$ & $\begin{array}{l}\text { Case } \\
\# 309\end{array}$ & $\begin{array}{l}\text { Should I combine Flat feelings } \\
\text { codes and Feeling detached or } \\
\text { unreal code? Are they kind of } \\
\text { similar? }\end{array}$ & $\begin{array}{l}\text { Numb / } \\
\text { Detached / } \\
\text { Lack of } \\
\text { energy or } \\
\text { interest }\end{array}$ & $\begin{array}{l}\text { there is a feeling of being "detached" } \\
\text { from things that would normally } \\
\text { produce extreme sadness. }\end{array}$ \\
\hline $12 / 30 / 2009$ & $\begin{array}{l}\text { Case } \\
\# 319\end{array}$ & $\begin{array}{l}\text { I don't have a code for shooting } \\
\text { pain / electrical zaps / brain zaps }\end{array}$ & $\begin{array}{l}\text { Zaps / } \\
\text { Tingling }\end{array}$ & $\begin{array}{l}\text { have some shooting pain in my head } \\
\text { that comes and goes. }\end{array}$ \\
\hline $12 / 31 / 2009$ & $\begin{array}{r}\text { Case } \\
\# 1081\end{array}$ & $\begin{array}{l}\text { I am noticing here a difference in } \\
\text { language use: these mental and } \\
\text { mood changes are called } \\
\text { "symptoms" whereas effects } \\
\text { called "side effects" are more } \\
\text { often physical effects (with only } \\
\text { a few mental/mood changes, } \\
\text { including agitation, restlessness, } \\
\text { and nervousness). To me, it } \\
\text { implies that worsening } \\
\text { mood/mental changes may be } \\
\text { part of the original problem } \\
\text { rather than or more so than an } \\
\text { effect of the drug. }\end{array}$ & $\begin{array}{l}\text { Talking with } \\
\text { doc / Doc } \\
\text { explained / } \\
\text { Consult doc }\end{array}$ & $\begin{array}{l}\text { Call your doctor at once if you have } \\
\text { any new or worsening symptoms such } \\
\text { as: }\end{array}$ \\
\hline $1 / 1 / 2010$ & $\begin{array}{l}\text { Case } \\
\# 333\end{array}$ & $\begin{array}{l}\text { Code: "Learned to live with it / } \\
\text { Learned to accept it" }\end{array}$ & $\begin{array}{l}\text { Learn to live } \\
\text { with it }\end{array}$ & $\begin{array}{l}\text { I have gained a bit of weight, but I dont } \\
\text { care. I am still pretty small just not } \\
\text { obsessed with being a size } 0 \text { anymore. }\end{array}$ \\
\hline
\end{tabular}




\begin{tabular}{|c|c|c|c|c|}
\hline Date & Case & Memo & Code & Consumer Comment \\
\hline $1 / 1 / 2010$ & $\begin{array}{l}\text { Case } \\
\# 348\end{array}$ & $\begin{array}{l}\text { Yes, this definitely illustrates } \\
\text { how Flat Feelings are the other } \\
\text { side of the Emotionally Stable } \\
\text { coin! }\end{array}$ & $\begin{array}{c}\text { Numb / } \\
\text { Detached / } \\
\text { Lack of } \\
\text { energy or } \\
\text { interest }\end{array}$ & $\begin{array}{l}\text { In a way, the medication has me on } \\
\text { such an even keel that it feels strange. } \\
\text { My reaction to things that would } \\
\text { normally upset me (and perhaps } \\
\text { should upset me) have been very } \\
\text { restrained. Pardon the simile, but I feel } \\
\text { like Spock on the old Star Trek series - } \\
\text { - very rationale and systematic, not at } \\
\text { all emotional. My therapist thought I } \\
\text { would not like being on Lexapro for } \\
\text { this very reason, but I guess it is } \\
\text { helping me work out issues at work in } \\
\text { a more productive way, and helping } \\
\text { me plan for a future career that is less } \\
\text { stress-inducing. }\end{array}$ \\
\hline $1 / 1 / 2010$ & $\begin{array}{l}\text { Case } \\
\# 349\end{array}$ & Code: "Learn to live with it" & $\begin{array}{c}\text { Learn to live } \\
\text { with it }\end{array}$ & $\begin{array}{l}\text { I do have a slight ringing in my ears-- } \\
\text { that I think is from the Lexapro -- but III } \\
\text { deal with that -- anything is better than } \\
\text { the panic and anxiety I had before. }\end{array}$ \\
\hline $1 / 1 / 2010$ & $\begin{array}{l}\text { Case } \\
\# 382\end{array}$ & $\begin{array}{l}\text { I've come across a couple } \\
\text { comments about taking Lexapro } \\
\text { during pregnancy, including this } \\
\text { one that Lex was a safer choice } \\
\text { than another med. There was } \\
\text { another comment within Lexapro } \\
\text { about a woman who did stay on } \\
\text { Lex during pregnancy and } \\
\text { breastfeeding and all turned out } \\
\text { well. Obviously, these would not } \\
\text { be contraindications, but what } \\
\text { should I code them as? }\end{array}$ & $\begin{array}{l}\text { Generic/oth } \\
\text { er drug } \\
\text { equivalence }\end{array}$ & $\begin{array}{l}\text { Is not as effective as Paxil. Switched } \\
\text { from Paxil thinking Lexapro was safer } \\
\text { since I want to get pregnant. }\end{array}$ \\
\hline $1 / 2 / 2010$ & $\begin{array}{l}\text { Case } \\
\# 162\end{array}$ & Code: Electrical tingling / shocks & $\begin{array}{l}\text { Zaps / } \\
\text { Tingling }\end{array}$ & $\begin{array}{l}\text { there has been this strange sensation } \\
\text { which I'm finding difficult to deal with. } \\
\text { It's in my arms mostly and feels like } \\
\text { there's a layer under my skin of } \\
\text { electrical pluses and when waking at } \\
3 \text { and } 4 \text { in the morning (still), I have } \\
\text { the desire to cut the skin and peel it } \\
\text { off, or cut the skin in a spiral round and } \\
\text { round the arm. It does go away } \\
\text { towards the end of the day (on most } \\
\text { days, but not today) and sometimes } \\
\text { it's around my head as well. }\end{array}$ \\
\hline
\end{tabular}




\begin{tabular}{|c|c|c|c|c|}
\hline Date & Case & Memo & Code & Consumer Comment \\
\hline $1 / 2 / 2010$ & $\begin{array}{l}\text { Case } \\
\# 272\end{array}$ & $\begin{array}{l}\text { I've been coming across this } \\
\text { idea of "I'm new to drugs / this is } \\
\text { only my } 1 \text { st (or } 2 \text { nd) drug so I } \\
\text { don't know what I should be } \\
\text { feeling or I have nothing to } \\
\text { compare this experience to" }\end{array}$ & OTHER & $\begin{array}{l}\text { I'm happy with the drug, but it was only } \\
\text { my second drug. }\end{array}$ \\
\hline $1 / 21 / 2010$ & $\begin{array}{l}\text { Case } \\
\# 172\end{array}$ & $\begin{array}{l}\text { Unsure how to categorize } \\
\text { "activating" as an effect. } \\
\text { Mar } 2010 \text { update: Coding this as } \\
\text { Energized / Euphoric because } \\
\text { activating is generally meant to } \\
\text { reflect a manic-type reaction. }\end{array}$ & $\begin{array}{c}\text { Energy } \\
\text { increased / } \\
\text { Euphoria / } \\
\text { Mania }\end{array}$ & $\begin{array}{l}\text { Lexapro (on its own) was activating for } \\
\text { me. My impression from lurking this } \\
\text { board for some time is that it is } \\
\text { activating for some others also. }\end{array}$ \\
\hline $1 / 24 / 2010$ & $\begin{array}{l}\text { Case } \\
\# 194\end{array}$ & Code as "Side Effect Trade-Off" & $\begin{array}{l}\text { Other } \\
\text { methods }\end{array}$ & $\begin{array}{l}\text { Dear me. Someone who'd pick thin } \\
\text { over sexual function. Think about that } \\
\text { for a minute. One is an appearance } \\
\text { issue. (You didn't say fit. You said thin. } \\
\text { Difference.) The other one is a basic } \\
\text { human drive and arguably integral to } \\
\text { primary bond relationships. It's like } \\
\text { Fernando from SNL all over again. It's } \\
\text { better to look good than to feel good. }\end{array}$ \\
\hline $1 / 24 / 2010$ & $\begin{array}{l}\text { Case } \\
\# 194\end{array}$ & $\begin{array}{l}\text { Code as "Learning to deal with } \\
\text { it/ live with it" }\end{array}$ & $\begin{array}{l}\text { Learn to live } \\
\text { with it }\end{array}$ & $\begin{array}{l}\text { I don't, personally, feel it's "fair" that } \\
\text { the medication that seems to be the } \\
\text { most effective for me is also the one } \\
\text { that's nibbling at my kidneys. I'm } \\
\text { taking it because it's preferable to the } \\
\text { alternative. (I'm also far past the belief } \\
\text { that the world is fair, though, so maybe } \\
\text { a certain amount of cynicism helps } \\
\text { here.) }\end{array}$ \\
\hline $1 / 24 / 2010$ & $\begin{array}{l}\text { Case } \\
\# 195\end{array}$ & $\begin{array}{l}\text { This comment relates to the } \\
\text { general idea of weighing pros } \\
\text { and cons, which is why I coded it } \\
\text { here (taking from "there are no } \\
\text { absolutes here") rather than All } \\
\text { in All or Side Effects Too Great. } \\
\text { Neither of the latter exactly fit. }\end{array}$ & YMMV & $\begin{array}{l}\text { Not taking antidepressants carries its } \\
\text { own set of very real risks, up to and } \\
\text { including death of mom and child. } \\
\text { There are no absolutes here. }\end{array}$ \\
\hline
\end{tabular}




\begin{tabular}{|c|c|c|c|c|}
\hline Date & Case & Memo & Code & Consumer Comment \\
\hline $1 / 24 / 2010$ & $\begin{array}{l}\text { Case } \\
\# 196\end{array}$ & $\begin{array}{l}\text { This is about mixing lexapro and } \\
\text { alcohol, but it's just the opposite } \\
\text { of a contraindication. He is } \\
\text { saying it's probably okay to mix } \\
\text { them and there should be no } \\
\text { interactions. I don't believe I } \\
\text { have a code that this would fall } \\
\text { under. }\end{array}$ & OTHER & $\begin{array}{l}\text { Because so many people drink, } \\
\text { especially depressed people, that the } \\
\text { drug companies do shitloads of tests } \\
\text { mixing the two. There is no } \\
\text { interaction, no change in the effects of } \\
\text { either drug, no nothing. Of course } \\
\text { doctors should tell people not to drink, } \\
\text { but a beer or two every now and then } \\
\text { has yet to affect anyone I've observed } \\
\text { or who has written about taking an } \\
\text { SSRI. }\end{array}$ \\
\hline $1 / 24 / 2010$ & $\begin{array}{l}\text { Case } \\
\# 204\end{array}$ & $\begin{array}{l}\text { I've coded this for a couple } \\
\text { comments relating to the effects } \\
\text { of the drug being different from } \\
\text { when the person took it at a } \\
\text { previous point in time. }\end{array}$ & $\begin{array}{l}\text { Poop Out / } \\
\text { Effects } \\
\text { Changed }\end{array}$ & $\begin{array}{l}\text { It does seem like a strange } \\
\text { paradoxical reaction...perhaps your } \\
\text { nervous system is in a more sensitzed } \\
\text { state this time? }\end{array}$ \\
\hline $1 / 24 / 2010$ & $\begin{array}{l}\text { Case } \\
\# 207\end{array}$ & $\begin{array}{l}\text { Another more general comment } \\
\text { about weighing pros and cons. } \\
\text { Even more specifically here, the } \\
\text { relativeness of effects as } \\
\text { desireable or undersireable. }\end{array}$ & $\begin{array}{l}\text { No or } \\
\text { limited } \\
\text { helpfulness } \\
\text { / It's not my } \\
\text { wonder drug }\end{array}$ & $\begin{array}{l}\text { I am not concerned, probably because } \\
\text { it hasn't gone on long enough to really } \\
\text { move from unpleasant but interesting } \\
\text { to outright irritating, but I rather wonder } \\
\text { how a person being treated for anxiety } \\
\text { is supposed to decide when not being } \\
\text { concerned is an undesirable side- } \\
\text { effect rather than a desired outcome. }\end{array}$ \\
\hline $1 / 24 / 2010$ & $\begin{array}{l}\text { Case } \\
\# 212\end{array}$ & Learn to live with it/ deal with it & $\begin{array}{l}\text { Learn to live } \\
\text { with it }\end{array}$ & $\begin{array}{l}\text { Once I had the crown replaced I just } \\
\text { got used to that side effect as the } \\
\text { combo worked so well for my moods } \\
\text { for several years. I was on } 300 \mathrm{mg} \\
\text { Wellbutrin and started at } 10 \mathrm{mg} \\
\text { Lexapro and later raised to } 20 \mathrm{mg} . \text { I } \\
\text { still have all my teeth and no long-term } \\
\text { damage that I'm aware of. }\end{array}$ \\
\hline $1 / 24 / 2010$ & $\begin{array}{l}\text { Case } \\
\# 232\end{array}$ & $\begin{array}{l}\text { Learn to live with; Also again this } \\
\text { idea of an effect as beneficial or } \\
\text { not is relative }\end{array}$ & $\begin{array}{l}\text { Learn to live } \\
\text { with it }\end{array}$ & $\begin{array}{l}\text { this is the one that still strikes me. It's } \\
\text { one I can live with and may even be a } \\
\text { beneficial effect depending on how } \\
\text { you think about it. }\end{array}$ \\
\hline $1 / 25 / 2010$ & $\begin{array}{l}\text { Case } \\
\# 233\end{array}$ & $\begin{array}{l}\text { A response to another user } \\
\text { stating that they expend more } \\
\text { calories than they take in, so } \\
\text { why do they continue to gain } \\
\text { weight? It goes against the laws } \\
\text { of science (according to this } \\
\text { user). The coded comment says } \\
\text { it would work that way but these } \\
\text { chemicals get in the way. }\end{array}$ & $\begin{array}{l}\text { Unsure } \\
\text { about } \\
\text { mechansim } \\
\text { / Other }\end{array}$ & $\begin{array}{l}\text { Lexapro User And Weight Gain Posted } \\
\text { by: Cyeic Sun } 16 \text { December } 2007 \\
\text { 5:35:35 GMT +0000 It would be } \\
\text { thermodynamics, and a simple case of } \\
\text { weighing energy intake against energy } \\
\text { expenditure, if those pesky genes and } \\
\text { neurochemicals didn't get involved. } \\
\text { Look up "leptin" and associated } \\
\text { molecules. }\end{array}$ \\
\hline
\end{tabular}




\begin{tabular}{|c|c|c|c|c|}
\hline Date & Case & Memo & Code & Consumer Comment \\
\hline $1 / 26 / 2010$ & $\begin{array}{l}\text { Case } \\
\# 620\end{array}$ & $\begin{array}{l}\text { Relieves an adverse effect } \\
\text { caused by another drug. }\end{array}$ & $\begin{array}{l}\text { Alleviates } \\
\text { side effect } \\
\text { from } \\
\text { another } \\
\text { drug }\end{array}$ & $\begin{array}{l}\text { Now I have switched to Seroquel as I } \\
\text { have thinning bones from amenorrhea } \\
\text { and within a month my prolactin levels } \\
\text { have dropped from } 3000 \text { to } 57 \text { and my } \\
\text { periods have returned. }\end{array}$ \\
\hline $1 / 26 / 2010$ & $\begin{array}{l}\text { Case } \\
\# 623\end{array}$ & $\begin{array}{l}\text { Maybe broaden the code "Going } \\
\text { back on meds" to also include } \\
\text { comments similar to "meds are } \\
\text { the solution for me" }\end{array}$ & $\begin{array}{l}\text { Going back } \\
\text { on meds / } \\
\text { Meds may } \\
\text { be the } \\
\text { solution }\end{array}$ & $\begin{array}{l}\text { Truth is, I function pretty well without } \\
\text { any of my meds, I just feel better with } \\
\text { them. I always need something for } \\
\text { sleep so while I'm taking something for } \\
\text { sleep I might as well take something } \\
\text { for everything else that ails me lol. }\end{array}$ \\
\hline $1 / 26 / 2010$ & $\begin{array}{l}\text { Case } \\
\# 624\end{array}$ & "Tweaking" & $\begin{array}{c}\text { Other } \\
\text { methods }\end{array}$ & $\begin{array}{l}\text { Things are really not all too bad right } \\
\text { now when you look at how bad they } \\
\text { have been in the past! I just think } \\
\text { maybe my meds need to be tweaked a } \\
\text { bit... }\end{array}$ \\
\hline $1 / 26 / 2010$ & $\begin{array}{l}\text { Case } \\
\# 632\end{array}$ & Use it PRN instead of daily & $\begin{array}{c}\text { Other } \\
\text { methods }\end{array}$ & $\begin{array}{l}\text { I had tried it once before back in } \\
\text { March and didn't like it because I was } \\
\text { taking it every night and made me into } \\
\text { a zombie during the day, but now I } \\
\text { take it only when feeling agitated at } \\
\text { night and once the zombification } \\
\text { wears off I feel a lot better. }\end{array}$ \\
\hline $1 / 26 / 2010$ & $\begin{array}{l}\text { Case } \\
\# 633\end{array}$ & "Side effect trade-off" & $\begin{array}{c}\text { Other } \\
\text { methods }\end{array}$ & $\begin{array}{l}\text { when i take it on and off, i dont sleep } \\
\text { enough, so im totally exhausted } \\
\text { physically, then i take it and crash, } \\
\text { sleep too much. }\end{array}$ \\
\hline $1 / 26 / 2010$ & $\begin{array}{l}\text { Case } \\
\# 633\end{array}$ & $\begin{array}{l}\text { Though this comment is } \\
\text { referring to how to tweak a med } \\
\text { combo, rather than just a } \\
\text { statement of what the combo is. } \\
\text { l've come across several general } \\
\text { statements about med combos } \\
\text { or cocktails needing to be } \\
\text { tweaked... }\end{array}$ & $\begin{array}{l}\text { My drug } \\
\text { combo / so } \\
\text { many damn } \\
\text { meds... }\end{array}$ & $\begin{array}{l}\text { was just reading other post in the } \\
\text { treatments for bipolar section... and } \\
\text { read up on the topamax sheet - if that } \\
\text { is a sleepy kind of drug, maybe that + } \\
\text { lamictal would be good and i wouldnt } \\
\text { need the seroquel as often. plus, it } \\
\text { would keep the headaches (which i } \\
\text { think are more like cluster than } \\
\text { migraine) more in check. }\end{array}$ \\
\hline
\end{tabular}




\begin{tabular}{|c|c|c|c|c|}
\hline Date & Case & Memo & Code & Consumer Comment \\
\hline $1 / 27 / 2010$ & $\begin{array}{l}\text { Case } \\
\# 638\end{array}$ & $\begin{array}{l}\text { Kind of similar to "Side Effect } \\
\text { Trade-Off", but not sure if that's } \\
\text { the best code here }\end{array}$ & $\begin{array}{l}\text { Other } \\
\text { methods }\end{array}$ & $\begin{array}{l}\text { After } 4 \text { years on seroquel I have been } \\
\text { having quite a bit of trouble getting my } \\
\text { sleep routine back into shape. The } \\
\text { thing is, the new medication that I am } \\
\text { on also has the initial side effect of } \\
\text { insomnia. I have been on that for } 7 \text { or } \\
8 \text { weeks now. I'm cool when it comes } \\
\text { to getting to sleep but I almost always } \\
\text { wake up at } 3 \text { am and sometimes can't } \\
\text { get back to sleep until about } 6 \text { am or } \\
\text { so. }\end{array}$ \\
\hline $1 / 27 / 2010$ & $\begin{array}{l}\text { Case } \\
\# 641\end{array}$ & $\begin{array}{l}\text { This is a general comment about } \\
\text { having to weigh side effects and } \\
\text { benefits }\end{array}$ & YMMV & $\begin{array}{l}\text { Sometimes I adjust to the side effects, } \\
\text { other times the side effects win out. }\end{array}$ \\
\hline $1 / 27 / 2010$ & $\begin{array}{l}\text { Case } \\
\# 641\end{array}$ & $\begin{array}{l}\text { "Waiting" to see if the effects will } \\
\text { last... }\end{array}$ & $\begin{array}{l}\text { Ride out the } \\
\text { storm / } \\
\text { Eventually } \\
\text { goes away / } \\
\text { Waiting }\end{array}$ & $\begin{array}{l}\text { If you are at a point where it feels good } \\
\text { then pat yourself on the back and say } \\
\text { a prayer that it lasts. Sometimes there } \\
\text { is no rhyme or reason for the change. }\end{array}$ \\
\hline $1 / 27 / 2010$ & $\begin{array}{l}\text { Case } \\
\# 647\end{array}$ & $\begin{array}{l}\text { This is in response to a user } \\
\text { stating the seroquel they've } \\
\text { been on for } 2 \text { years is "bombing } \\
\text { out." The present comment is } \\
\text { advising that they plan what they } \\
\text { could do next. }\end{array}$ & $\begin{array}{l}\text { Poop Out / } \\
\text { Effects } \\
\text { Changed }\end{array}$ & $\begin{array}{l}\text { Q's Bombing Out? Posted by: seaview } \\
\text { Fri } 2 \text { January } 2009 \text { 15:48:42 GMT } \\
+0000 \text { Yes. It may not hit, but better } \\
\text { plan what your next moves will be if it } \\
\text { does. }\end{array}$ \\
\hline $1 / 27 / 2010$ & $\begin{array}{l}\text { Case } \\
\# 647\end{array}$ & $\begin{array}{l}\text { Is the drug "bombing out" or is it } \\
\text { life stresses getting to me? (this } \\
\text { is the question the previous user } \\
\text { poses) }\end{array}$ & $\begin{array}{l}\text { Drug or } \\
\text { Something } \\
\text { Else? }\end{array}$ & $\begin{array}{l}\text { SEE QUOTE. Possibly. Possibly not. } \\
\text { (I'm dead helpful sometimes, me.) But } \\
\text { you know you've had a "fair bit to deal } \\
\text { with" recently, and you've moved, and } \\
\text { it's the time of year for festilities that } \\
\text { unbalance all of us in some way. All } \\
\text { adds to the mix. }\end{array}$ \\
\hline $1 / 27 / 2010$ & $\begin{array}{l}\text { Case } \\
\# 647\end{array}$ & $\begin{array}{l}\text { This refers to having or making } \\
\text { plans for ongoing well-being } \\
\text { and/or stability. Kind of reminds } \\
\text { me of users talking about } \\
\text { needing to tweak their cocktails, } \\
\text { or trying to think ahead or plan } \\
\text { ahead for what they anticipate } \\
\text { may be about to happen. }\end{array}$ & $\begin{array}{l}\text { Making a } \\
\text { plan / Just } \\
\text { in case... }\end{array}$ & $\begin{array}{l}\text { It's good that all your support eggs } \\
\text { aren't in one basket. That's a clumsy } \\
\text { way to put it, sorry - I guess I mean } \\
\text { that it's good that you have a GP and } \\
\text { meds and people acting as carers and } \\
\text { some maintenance / crisis plans. }\end{array}$ \\
\hline
\end{tabular}




\begin{tabular}{|c|c|c|c|c|}
\hline Date & Case & Memo & Code & Consumer Comment \\
\hline $1 / 27 / 2010$ & $\begin{array}{l}\text { Case } \\
\# 647\end{array}$ & Learn to live with it/deal with it & $\begin{array}{l}\text { Learn to live } \\
\text { with it }\end{array}$ & $\begin{array}{l}\text { a need to eat masses of carbohydrate. } \\
\text { Far more than my body could } \\
\text { genuinely need for day-to-day } \\
\text { functioning. I could easily add another } \\
10 \text { pounds in a week or so. I don't like } \\
\text { this side-effect, but I don't think too } \\
\text { much about it. }\end{array}$ \\
\hline $1 / 27 / 2010$ & $\begin{array}{l}\text { Case } \\
\# 649\end{array}$ & $\begin{array}{l}\text { Compare data to code here - } \\
\text { maybe needs to go in Making a } \\
\text { plan/Just in case.... I think that's } \\
\text { where I've put other comments } \\
\text { about keeping around } \\
\text { emergency meds }\end{array}$ & $\begin{array}{l}\text { Making a } \\
\text { plan / Just } \\
\text { in case... }\end{array}$ & $\begin{array}{l}\text { Plus, a lot of people don't really like } \\
\text { using AAP's as long term, } \\
\text { maintenance therapy, but see them as } \\
\text { best used only as short term, } \\
\text { emergency meds. }\end{array}$ \\
\hline $1 / 27 / 2010$ & $\begin{array}{l}\text { Case } \\
\# 659\end{array}$ & $\begin{array}{l}\text { Again, Making A Back-Up Plan } \\
\text { or Strategy }\end{array}$ & $\begin{array}{l}\text { Making a } \\
\text { plan / Just } \\
\text { in case... }\end{array}$ & $\begin{array}{l}\text { No, haven't tried Topa, but it is my } \\
\text { back up plan if I get the rash from } \\
\text { lamictal. See if he'll give it to you, } \\
\text { because it seems like any weight-gain } \\
\text { med may trigger an e/d episode. }\end{array}$ \\
\hline $1 / 27 / 2010$ & $\begin{array}{l}\text { Case } \\
\# 666\end{array}$ & $\begin{array}{l}\text { The comments by this user } \\
\text { seem suspicious to me - like this } \\
\text { info is just copied from a PI } \\
\text { sheet or some other source, and } \\
\text { a couple spelling/grammar } \\
\text { errors are thrown in to make it } \\
\text { appear authentic. Don't know... }\end{array}$ & $\begin{array}{l}\text { Symptoms } \\
\text { reduced / } \\
\text { improved }\end{array}$ & $\begin{array}{l}\text { it works at low doses as it is the most } \\
\text { sedating of the anti-pyyscotics so will } \\
\text { calm your anxiety and help to stabilise } \\
\text { your depression if your prone to } \\
\text { cycling. }\end{array}$ \\
\hline $1 / 27 / 2010$ & $\begin{array}{l}\text { Case } \\
\# 679\end{array}$ & $\begin{array}{l}\text { Having a back-up plan or } \\
\text { strategy }\end{array}$ & $\begin{array}{l}\text { Making a } \\
\text { plan / Just } \\
\text { in case... }\end{array}$ & $\begin{array}{l}\text { It's good that you have a plan and it } \\
\text { sounds like you have good } \\
\text { professionals too. }\end{array}$ \\
\hline $1 / 27 / 2010$ & $\begin{array}{l}\text { Case } \\
\# 682\end{array}$ & $\begin{array}{l}\text { Other methods to make it okay } \\
\text { to have a few drinks while on } \\
\text { seroquel }\end{array}$ & $\begin{array}{c}\text { Other } \\
\text { methods }\end{array}$ & $\begin{array}{l}\text { Alcohol Effects/ Libido On Seroquel } \\
\text { Posted by: therapeuticbrigg84 Sun } 14 \\
\text { December } 200822: 13: 15 \text { GMT }+0000 \\
\text { I guess you're right in that it takes } \\
\text { staying out late out of the equation, but } \\
\text { I don't think that one night a week of } \\
\text { staying out late is bad on this drug. as } \\
\text { I would end up taking it at a later time } \\
\text { maybe at } 1 \text { am or } 2 \text { am instead of my } \\
\text { regular } 12 \text { am drop- basically cuz I'm } \\
\text { pretty much a nightowl anyways. }\end{array}$ \\
\hline $1 / 28 / 2010$ & $\begin{array}{l}\text { Case } \\
\# 686\end{array}$ & $\begin{array}{l}\text { Change to blood sugar / glucose } \\
\text { changes }\end{array}$ & $\begin{array}{l}\text { Blood sugar } \\
\text { high / } \\
\text { Diabetes }\end{array}$ & $\begin{array}{l}\text { And jooks right about the glocose } \\
\text { levels. Mine shot down when I woke } \\
\text { up from a seroquel saturated slumber. }\end{array}$ \\
\hline
\end{tabular}




\begin{tabular}{|c|c|c|c|c|}
\hline Date & Case & Memo & Code & Consumer Comment \\
\hline $1 / 28 / 2010$ & $\begin{array}{l}\text { Case } \\
\# 715\end{array}$ & $\begin{array}{l}\text { Relieves adverse effect caused } \\
\text { by another drug }\end{array}$ & $\begin{array}{l}\text { Alleviates } \\
\text { side effect } \\
\text { from } \\
\text { another } \\
\text { drug }\end{array}$ & $\begin{array}{l}\text { im currently suffering from lamictal } \\
\text { induced psychosis that wont go away } \\
\text { with zyprexa, clozapine (getting worse) } \\
\text { and bit better on seroquel and abilify }\end{array}$ \\
\hline $1 / 28 / 2010$ & $\begin{array}{l}\text { Case } \\
\# 717\end{array}$ & $\begin{array}{l}\text { Previous user stated that their } \\
\text { doctor said } 75 \mathrm{mg} \text { of Seroquel } \\
\text { will not make them gain weight, } \\
\text { and was asking the forum if this } \\
\text { was true... }\end{array}$ & $\begin{array}{l}\text { Trusting the } \\
\text { doctor? }\end{array}$ & $\begin{array}{l}\text { So maybe, maybe not. Seroquel is } \\
\text { definately one of the AAP associated } \\
\text { with weight gain , so perhapse your } \\
\text { doctor was trying to reasure you, or.... }\end{array}$ \\
\hline $1 / 28 / 2010$ & $\begin{array}{l}\text { Case } \\
\# 717\end{array}$ & $\begin{array}{l}\text { General comment about the } \\
\text { user having to weigh benefits } \\
\text { and side effects }\end{array}$ & YMMV & $\begin{array}{l}\text { Yes, seroquel has side effects, but all } \\
\text { medications do, you're not guarenteed } \\
\text { to get them - it seems you've been on } \\
\text { the medication for } 5 \text { years without } \\
\text { problems. }\end{array}$ \\
\hline $1 / 31 / 2010$ & $\begin{array}{l}\text { Case } \\
\# 532\end{array}$ & $\begin{array}{l}\text { I see this a lot - the "trade-off" - } \\
\text { this concept *could* fall under } \\
\text { the 'Limited Benefit' code. }\end{array}$ & $\begin{array}{c}\text { Extreme } \\
\text { sleepiness / } \\
\text { Tired }\end{array}$ & $\begin{array}{l}\text { Secondly, it makes me way too } \\
\text { sleepy... after an hour after I take the } \\
\text { medication I'm usually unable to move } \\
\text { about without tripping and falling. On } \\
\text { the flipside, without it I can't sleep, and } \\
\text { if I do sleep, it's like a } 2 \text { hour nap. Not } \\
\text { fun. }\end{array}$ \\
\hline $1 / 31 / 2010$ & $\begin{array}{l}\text { Case } \\
\# 538\end{array}$ & $\begin{array}{l}\text { I coded this as "limited benefit/ok } \\
\text { but not great" because this user } \\
\text { is weighing the benefits and } \\
\text { costs, but has not yet reached a } \\
\text { conclusion - other than the fact } \\
\text { that the drug isn't 'perfect' }\end{array}$ & $\begin{array}{l}\text { No or } \\
\text { limited } \\
\text { helpfulness } \\
\text { / It's not my } \\
\text { wonder drug }\end{array}$ & $\begin{array}{l}\text { have been on } 400 \text { mgs a day for a } \\
\text { month.dont know yet if the } \\
\text { benifits(less anxious,less easily } \\
\text { upset,moodswings lessen )is worth } \\
\text { the side effects. }\end{array}$ \\
\hline $1 / 31 / 2010$ & $\begin{array}{l}\text { Case } \\
\# 546\end{array}$ & $\begin{array}{l}\text { Not sure whether to code this } \\
\text { here or under "I'm done / I want } \\
\text { off". I put it here because there } \\
\text { is some element of weighing the } \\
\text { benefits and costs of the drug } \\
\text { here (as it DOES help them } \\
\text { sleep, but it may not be worth } \\
\text { the other effects). }\end{array}$ & $\begin{array}{l}\text { No or } \\
\text { limited } \\
\text { helpfulness } \\
\text { / It's not my } \\
\text { wonder drug }\end{array}$ & $\begin{array}{l}\text { Seems to help alittle but really want to } \\
\text { get off this stuff - only thing is - it does } \\
\text { help me sleep. }\end{array}$ \\
\hline $2 / 1 / 2010$ & $\begin{array}{l}\text { Case } \\
\# 557\end{array}$ & $\begin{array}{l}\text { Another one where they know } \\
\text { they have to weigh pros and } \\
\text { cons, but did not reach a } \\
\text { conclusion within this review. I } \\
\text { put it here because it's an } \\
\text { acknowledgement of "limited } \\
\text { benefit" - in that the drug } \\
\text { partially solves their problem, } \\
\text { but causes other problems. }\end{array}$ & $\begin{array}{l}\text { No or } \\
\text { limited } \\
\text { helpfulness } \\
\text { / It's not my } \\
\text { wonder drug }\end{array}$ & $\begin{array}{l}\text { So I ask myself: fat and happy or thin } \\
\text { and crazy? Hmmmmmm... I have } \\
\text { gained 30lbs since } 12 / 06 \text { and I know } \\
\text { that cannot ALL be my fault! It's kinda } \\
\text { bad when you have to choose } \\
\text { between your mental and physical } \\
\text { health. It is a tough choice to make. }\end{array}$ \\
\hline
\end{tabular}




\begin{tabular}{|c|c|c|c|c|}
\hline Date & Case & Memo & Code & Consumer Comment \\
\hline $2 / 1 / 2010$ & $\begin{array}{l}\text { Case } \\
\# 563\end{array}$ & $\begin{array}{l}\text { weight gain isn't a "real" side } \\
\text { effect because it's just a given } \\
\text { effect for any antipsychotic }\end{array}$ & "Side Effect" & $\begin{array}{l}\text { Long term use has not had any real } \\
\text { side effects except for the traditional } \\
\text { weight gain with an anti-psychotic. }\end{array}$ \\
\hline $2 / 1 / 2010$ & $\begin{array}{l}\text { Case } \\
\# 568\end{array}$ & $\begin{array}{l}\text { I see this over and over again - } \\
\text { being tired or fatigued, but also } \\
\text { liking this same effect for it's } \\
\text { help in sleeping. The same } \\
\text { effect is both good and bad } \\
\text { depending on what the user } \\
\text { needs at a certain time or in a } \\
\text { certain context. I need to be sure } \\
\text { to read through all the sleep } \\
\text { codes, and may need to merge } \\
\text { the "helps sleep" with the "tired" } \\
\text { code - as they are really the } \\
\text { same effect, just a different } \\
\text { interpretation given. }\end{array}$ & $\begin{array}{l}\text { Extreme } \\
\text { sleepiness / } \\
\text { Tired }\end{array}$ & Fatigue (good for sleep though) \\
\hline $2 / 1 / 2010$ & $\begin{array}{l}\text { Case } \\
\# 570\end{array}$ & $\begin{array}{l}\text { This is almost another code for } \\
\text { sleeping: "extreme sleeping". It } \\
\text { doesn't quite fit within "helps me } \\
\text { sleep" and it's not "excessive } \\
\text { sleeping", but users most often } \\
\text { describe it as "extreme" sleeping }\end{array}$ & $\begin{array}{l}\text { Extreme } \\
\text { sleepiness / } \\
\text { Tired }\end{array}$ & $\begin{array}{l}\text { This pill feels very strong as it truly } \\
\text { knocks me out after taking at night. I } \\
\text { wonder if I could wake if the house } \\
\text { was on fire?!? SIDE EFFECTS } \\
\text { extreme sleeping within half hour at } \\
\text { night taking } 300 \mathrm{mg} \text {. }\end{array}$ \\
\hline $2 / 1 / 2010$ & $\begin{array}{l}\text { Case } \\
\# 584\end{array}$ & $\begin{array}{l}\text { I am coding any instance that a } \\
\text { person has, may have, or is } \\
\text { warning others of the risk of } \\
\text { diabetes. I decided to code this } \\
\text { way because I code 'expert' } \\
\text { sites based on warnings of side } \\
\text { effects, i.e., WebMD lists a } \\
\text { bunch of side effects and I code } \\
\text { them as side effects. I thought it } \\
\text { was only fair to treat consumer } \\
\text { sites the same way, so even if it } \\
\text { is not a personally experienced } \\
\text { effect, but something that they } \\
\text { list as a possible effect, I am } \\
\text { coding it as an effect. }\end{array}$ & $\begin{array}{l}\text { Blood sugar } \\
\text { high / } \\
\text { Diabetes }\end{array}$ & $\begin{array}{l}\text { I have heard there is a risk for } \\
\text { diabetes with seroquel and blood tests } \\
\text { should be required periodically }\end{array}$ \\
\hline $2 / 1 / 2010$ & $\begin{array}{l}\text { Case } \\
\# 590\end{array}$ & $\begin{array}{l}\text { Limited benefit because of the } \\
\text { "trade-off" }\end{array}$ & $\begin{array}{l}\text { No or } \\
\text { limited } \\
\text { helpfulness } \\
\text { / It's not my } \\
\text { wonder drug }\end{array}$ & $\begin{array}{l}\text { Why does there always have to be a } \\
\text { trade-off with medications? You either } \\
\text { do well physically without, but suffer } \\
\text { mentally - or you take the meds, feel } \\
\text { well menatally, but pudge out! If these } \\
\text { med-researchers ever figure out the } \\
\text { metabolism/weight gain/endocrine } \\
\text { problems, they would hit the jack-pot!!! } \\
\text { GET IT RIGHT DOCS. }\end{array}$ \\
\hline
\end{tabular}




\begin{tabular}{|c|c|c|c|c|}
\hline Date & Case & Memo & Code & Consumer Comment \\
\hline $2 / 1 / 2010$ & $\begin{array}{l}\text { Case } \\
\# 768\end{array}$ & $\begin{array}{l}\text { For so many people, the same } \\
\text { effect is both good and bad at } \\
\text { the same time. }\end{array}$ & $\begin{array}{l}\text { Numb / } \\
\text { Detached / } \\
\text { Lack of } \\
\text { energy or } \\
\text { interest }\end{array}$ & $\begin{array}{l}\text { I was taking a lower dosage of } \\
\text { Seroquel for over a year before and } \\
\text { complained about the 'doziness.' I } \\
\text { didn't really care about day-to-day stuff } \\
\text { like mounting bills and housework, etc. } \\
\text { On the other hand, I didn't get anxiety } \\
\text { attacks either. }\end{array}$ \\
\hline $2 / 2 / 2010$ & $\begin{array}{l}\text { Case } \\
\# 803\end{array}$ & $\begin{array}{l}\text { I'm taking this as an effect of the } \\
\text { drug - this user was able to cut } \\
\text { down on alcohol use as a result } \\
\text { of the seroquel working so well. }\end{array}$ & $\begin{array}{l}\text { Other Misc } \\
\text { Effects }\end{array}$ & $\begin{array}{l}\text { I have cut back on my alcohol use to a } \\
\text { much more acceptable level }\end{array}$ \\
\hline $2 / 2 / 2010$ & $\begin{array}{l}\text { Case } \\
\# 810\end{array}$ & $\begin{array}{l}\text { I coded this segment twice - as } \\
\text { 'helps me sleep' and 'other'. The } \\
\text { 'Other' is because it relieves an } \\
\text { adverse effect of another drug }\end{array}$ & $\begin{array}{l}\text { Alleviates } \\
\text { side effect } \\
\text { from } \\
\text { another } \\
\text { drug }\end{array}$ & $\begin{array}{l}\text { Seroquel has enabled me to take my } \\
\text { antidepressants, which cause } \\
\text { insomnia. }\end{array}$ \\
\hline $2 / 2 / 2010$ & $\begin{array}{l}\text { Case } \\
\# 811\end{array}$ & Learn to live with it/deal with it & $\begin{array}{l}\text { Learn to live } \\
\text { with it }\end{array}$ & $\begin{array}{l}\text { I hope to find a cure for the eating } \\
\text { thing...if not, I'll just have to be heavy }\end{array}$ \\
\hline $2 / 2 / 2010$ & $\begin{array}{l}\text { Case } \\
\# 823\end{array}$ & $\begin{array}{l}\text { Relieves adverse effect of } \\
\text { another drug }\end{array}$ & $\begin{array}{l}\text { Alleviates } \\
\text { side effect } \\
\text { from } \\
\text { another } \\
\text { drug }\end{array}$ & $\begin{array}{l}\text { It always beats my insomnia, which is } \\
\text { caused by my Bipolar and other the } \\
\text { medications that I'm on. }\end{array}$ \\
\hline $2 / 2 / 2010$ & $\begin{array}{l}\text { Case } \\
\# 826\end{array}$ & helps with IBS & $\begin{array}{l}\text { Other Misc } \\
\text { Effects }\end{array}$ & It helps w/hypomanic, sleep, IBS \\
\hline $2 / 2 / 2010$ & $\begin{array}{l}\text { Case } \\
\# 862\end{array}$ & $\begin{array}{l}\text { This is a combo of appetite and } \\
\text { memory loss or sleep-walking - } \\
\text { "sleep eating". Good term to use } \\
\text { as a code or as a term to } \\
\text { describe users' experience. } \\
\text { General Note, two terms } \\
\text { invented by users: } \\
\text { sleep eating and } \\
\text { extreme sleeping }\end{array}$ & $\begin{array}{l}\text { Increase } \\
\text { appetite / } \\
\text { cravings }\end{array}$ & $\begin{array}{l}\text { Thanks to the person who mentioned } \\
\text { the sleep eating! I began doing that } \\
\text { suddenly and did not know it was the } \\
\text { Seroquel }\end{array}$ \\
\hline $2 / 2 / 2010$ & $\begin{array}{l}\text { Case } \\
\# 870\end{array}$ & with sleep walking & $\begin{array}{l}\text { Memory } \\
\text { loss }\end{array}$ & $\begin{array}{l}\text { Taking } 600 \mathrm{mg} \text { every night. Sometime } \\
\text { during the night I went into my } \\
\text { livingroom and somehow wrecked my } \\
\text { whole apt.! I woke up on the rug and } \\
\text { realized what I had done. }\end{array}$ \\
\hline
\end{tabular}




\begin{tabular}{|c|c|c|c|c|}
\hline Date & Case & Memo & Code & Consumer Comment \\
\hline 2/2/2010 & $\begin{array}{l}\text { Case } \\
\# 872\end{array}$ & $\begin{array}{l}\text { Another case of weighing pros } \\
\text { and cons without reaching a real } \\
\text { conclusion, other than the drug } \\
\text { fixes some problems and causes } \\
\text { many others. }\end{array}$ & $\begin{array}{l}\text { No or } \\
\text { limited } \\
\text { helpfulness } \\
\text { / It's not my } \\
\text { wonder drug }\end{array}$ & $\begin{array}{l}\text { He is closely monitored, but even with } \\
\text { that he is now experiencing health } \\
\text { problems that are a direct result of this } \\
\text { med. His triglicerides are off the chart } \\
\text {, blood-pressure is too low, sugar } \\
\text { high, and occasional chest pains . } \\
\text { Since we have been through most of } \\
\text { the available meds for his dissorders, } \\
\text { and he is relative stabile,it has been } \\
\text { decided that taking him off of the meds } \\
\text { will cause him to crash. I am in the } \\
\text { process of working with the doctors, } \\
\text { med and pcp, to decide what to do } \\
\text { before... I'm not sure what could } \\
\text { happen to him, but I was told his } \\
\text { situation is dangerous. I know it isn't } \\
\text { fair that there are not better meds out } \\
\text { there, or ones that the side affects } \\
\text { wouldn't kill you, so what is a mother } \\
\text { to do? Either way it seems to me that } \\
\text { my son is the looser; breakdown- } \\
\text { suicide attempts or diabetes, heart } \\
\text { disease, or stroke. What would you } \\
\text { do????? }\end{array}$ \\
\hline $2 / 3 / 2010$ & $\begin{array}{r}\text { Case } \\
\# 1002\end{array}$ & $\begin{array}{l}\text { I am coding books as 'academic' } \\
\text { though I may need to go back } \\
\text { and pull these out into their own } \\
\text { category }\end{array}$ & $\begin{array}{l}\text { Academic } \\
\text { Journal / } \\
\text { "Research } \\
\text { shows..." }\end{array}$ & $\begin{array}{l}\text { have you ever read---The Looney Bin } \\
\text { Trip? By Kate Millet...good read, from } \\
\text { a very intellectual woman who didn't } \\
\text { like her lithium.... but I am not anti- } \\
\text { lithium, }\end{array}$ \\
\hline $2 / 3 / 2010$ & $\begin{array}{r}\text { Case } \\
\# 1055\end{array}$ & $\begin{array}{l}\text { User is stating that they want to } \\
\text { go from taking their } \\
\text { antipsychotic everyday as } \\
\text { maintenance treatment to an as- } \\
\text { needed/PRN basis. }\end{array}$ & $\begin{array}{l}\text { Dose } \\
\text { related } \\
\text { issues / } \\
\text { advice }\end{array}$ & $\begin{array}{l}\text { let me taper off the risperidone and let } \\
\text { me try something else to stabilize my } \\
\text { mood, and just have the anti-psychotic } \\
\text { as a safety net. }\end{array}$ \\
\hline $2 / 3 / 2010$ & $\begin{array}{r}\text { Case } \\
\# 1055\end{array}$ & $\begin{array}{l}\text { Link to an MSN article about } \\
\text { risperidone being on the list of } \\
\text { the top } 10 \text { most dangerous } \\
\text { drugs }\end{array}$ & Internet & $\begin{array}{l}\text { Wow, thanks dionysian. Definitely } \\
\text { helps. I emailed that link to the } \\
\text { psychiatrist too. }\end{array}$ \\
\hline $2 / 6 / 2010$ & $\begin{array}{l}\text { Case } \\
\# 977\end{array}$ & Learn to live with it / deal with it & $\begin{array}{c}\text { Learn to live } \\
\text { with it }\end{array}$ & $\begin{array}{l}\text { He said he would be willing to work } \\
\text { with me on changing meds...but } \\
\text { recommended that I just deal with the } \\
\text { tremor, unless it gets worse. }\end{array}$ \\
\hline $2 / 8 / 2010$ & $\begin{array}{l}\text { Case } \\
\# 986\end{array}$ & $\begin{array}{l}\text { General comment about } \\
\text { weighing benefits vs negative } \\
\text { effects }\end{array}$ & $\begin{array}{l}\text { No or } \\
\text { limited } \\
\text { helpfulness } \\
\text { / It's not my } \\
\text { wonder drug }\end{array}$ & $\begin{array}{l}\text { Perhaps list the effects of the medicine } \\
\text { you are taking. If the effects do not line } \\
\text { up with your goal or the negative } \\
\text { effects outweigh the positive then this } \\
\text { is a very good case for change. }\end{array}$ \\
\hline
\end{tabular}




\begin{tabular}{|c|c|c|c|c|}
\hline Date & Case & Memo & Code & Consumer Comment \\
\hline $2 / 8 / 2010$ & $\begin{array}{l}\text { Case } \\
\# 986\end{array}$ & $\begin{array}{l}\text { PBS show on tv. I coded it here } \\
\text { because I also coded 'books' } \\
\text { here, and may separate them } \\
\text { out into } 1 \text { category later }\end{array}$ & $\begin{array}{l}\text { Academic } \\
\text { Journal / } \\
\text { "Research } \\
\text { shows..." }\end{array}$ & $\begin{array}{l}\text { PBS Frontline, Tuesday Jan8 The } \\
\text { Medicated Child Posted by: Inel just } \\
\text { saw it... it was very anti-climactic for } \\
\text { me. a little too soft-core. }\end{array}$ \\
\hline $2 / 8 / 2010$ & $\begin{array}{l}\text { Case } \\
\# 991\end{array}$ & $\begin{array}{l}\text { In this 'Other' category, I've } \\
\text { come across several times this } \\
\text { idea of "I wish, I wish, I wish..." }\end{array}$ & OTHER & $\begin{array}{l}\text { Would'nt it be nice to be happy and } \\
\text { normal without having to swallow all of } \\
\text { this damn rat poison??!!! }\end{array}$ \\
\hline $2 / 8 / 2010$ & $\begin{array}{r}\text { Case } \\
\# 1013\end{array}$ & $\begin{array}{l}\text { Wants to quit meds, but stays on } \\
\text { them because of fear of what } \\
\text { might happen without them.... Is } \\
\text { that coded here, or as a } \\
\text { Dependence issue? }\end{array}$ & $\begin{array}{l}\text { Drugs won't } \\
\text { work / I'm } \\
\text { done / I } \\
\text { don't need it }\end{array}$ & $\begin{array}{l}\text { Wow i know exatly what you're talking } \\
\text { about. I haven't even been able to } \\
\text { make it to trying to go down on my } \\
\text { meds but i think about it all the time. } \\
\text { I'm just so afraid of my symptoms } \\
\text { returning. without meds, mine are } \\
\text { quite strong and this scares the } \\
\text { daylights out of me. }\end{array}$ \\
\hline $2 / 9 / 2010$ & $\begin{array}{r}\text { Case } \\
\# 1036\end{array}$ & $\begin{array}{l}\text { Not really 'drugs won't work,' but } \\
\text { a comment about the limited and } \\
\text { appropriate use of drugs... }\end{array}$ & $\begin{array}{l}\text { Drugs won't } \\
\text { work / I'm } \\
\text { done / I } \\
\text { don't need it }\end{array}$ & $\begin{array}{l}\text { People who are in severe psychotic } \\
\text { states or in states of self-injuring or } \\
\text { injuring others, or who are totally } \\
\text { unable to function often need to be } \\
\text { medicated. But the medication should } \\
\text { be short-term, at as low a dosage as } \\
\text { possible, with polypharmacy avoided } \\
\text { (no more than two drugs together), } \\
\text { and the patients should be titrated off } \\
\text { properly as soon as they are given the } \\
\text { proper modalities to support healing at } \\
\text { the root level of the condition, which } \\
\text { can be orthomolecular, energetic, } \\
\text { psychotherapeutic, probably several } \\
\text { things in combination--in a caring } \\
\text { environment that embraces nature. } \\
\text { However, our society is not set up in } \\
\text { such a way as to permit this. }\end{array}$ \\
\hline $2 / 9 / 2010$ & $\begin{array}{r}\text { Case } \\
\# 1040\end{array}$ & "Side effect trade-off" & $\begin{array}{c}\text { Other } \\
\text { methods }\end{array}$ & $\begin{array}{l}\text { My doc added zonegran to the } \\
\text { zyprexa to try and mediate the weight } \\
\text { gain. Makes me very sleepy though. }\end{array}$ \\
\hline $2 / 10 / 2010$ & $\begin{array}{r}\text { Case } \\
\# 1085\end{array}$ & $\begin{array}{l}\text { "Warnings" - not "side effects" - } \\
\text { but I had no where else to code } \\
\text { this term }\end{array}$ & "Side Effect" & $\begin{array}{l}\text { What warnings do you have for } \\
\text { Seroquel (Quetiapine)? The following } \\
\text { warnings are available for this } \\
\text { medication: May impair driving. Do } \\
\text { not drink alcohol. Obtain advice for } \\
\text { OTCs. May cause drowsiness. } \\
\text { Check with your doctor before } \\
\text { becoming pregnant. }\end{array}$ \\
\hline
\end{tabular}




\begin{tabular}{|c|c|c|c|c|}
\hline Date & Case & Memo & Code & Consumer Comment \\
\hline $2 / 10 / 2010$ & $\begin{array}{r}\text { Case } \\
\# 1086\end{array}$ & $\begin{array}{l}\text { "used with or without other } \\
\text { medications" }\end{array}$ & OTHER & $\begin{array}{l}\text { This medication is used with or without } \\
\text { other medications to treat certain } \\
\text { mental/mood conditions }\end{array}$ \\
\hline $2 / 11 / 2010$ & $\begin{array}{r}\text { Case } \\
\# 1083\end{array}$ & $\begin{array}{l}\text { I coded this here because of the } \\
\text { last } 2 \text { sentences.... which } \\
\text { comment on the lack of } \\
\text { transparency in both published } \\
\text { articles and the PI sheet. Jarod } \\
\text { here is trying to piece these } \\
\text { incomplete bits of info together }\end{array}$ & $\begin{array}{l}\text { Three } \\
\text { percent my } \\
\text { ass! }\end{array}$ & $\begin{array}{l}\text { I could find a study } \\
\text { http://www.ncbi.nlm.nih.gov/entrez/que } \\
\text { ry.fcgi?cmd=Retrieve\&db=PubMed\&lis } \\
\text { t_uids=14744167\&dopt=Abstractindica } \\
\text { ting that Lexapro (escitalopram } \\
\text { oxalate) is good to prevent relapses of } \\
\text { major depressive disorder when } \\
\text { compared to a placebo. Close to } \\
75 \% \text { of the people taking Lexapro } \\
\text { (escitalopram oxalate) after } 4-6 \\
\text { months were still not depressed, vs. } \\
60 \% \text { taking the wonder drug Placebo. } \\
\text { Unfortunately we don't get to see how } \\
\text { their MADRS scores were. I suspect } \\
\text { this was the fourth trial mentioned in } \\
\text { the PI sheet. }\end{array}$ \\
\hline $2 / 11 / 2010$ & $\begin{array}{r}\text { Case } \\
\# 1083\end{array}$ & $\begin{array}{l}\text { Relieved an adverse effect of } \\
\text { another drug }\end{array}$ & $\begin{array}{l}\text { Alleviates } \\
\text { side effect } \\
\text { from } \\
\text { another } \\
\text { drug }\end{array}$ & $\begin{array}{l}\text { I've found one reference to a single } \\
\text { case } \\
\text { studyhttp://www.ncbi.nlm.nih.gov/entre } \\
\text { z/query.fcgi?cmd=Retrieve\&db=PubM } \\
\text { ed\&list_uids=14660288\&dopt=Abstrac } \\
\text { t where someone overcame the } \\
\text { sexual side effects of } \\
\text { Prozachttp://www.crazymeds.us/proza } \\
\text { c.html (fluoxetine hydrochloride) by } \\
\text { switching to Lexapro (escitalopram } \\
\text { oxalate). }\end{array}$ \\
\hline $2 / 12 / 2010$ & $\begin{array}{r}\text { Case } \\
\# 1084\end{array}$ & Agitation & $\begin{array}{c}\text { Other } \\
\text { reason / } \\
\text { diagnosis }\end{array}$ & $\begin{array}{l}\text { If you're agitated, jumping out of your } \\
\text { skin and you just can't sleep, then } \\
\text { Seroquel (quetiapine fumarate) might } \\
\text { be just what you're looking for. }\end{array}$ \\
\hline $2 / 12 / 2010$ & $\begin{array}{r}\text { Case } \\
\# 1084\end{array}$ & $\begin{array}{l}\text { I need to look at the "zmobie" } \\
\text { codes, which I have put all under } \\
\text { "cognitive slowing, spacey, } \\
\text { dopey". Jarod equates zombie } \\
\text { with "flat feelings, lack of } \\
\text { interest". Perhaps I need to } \\
\text { merge these codes?? }\end{array}$ & $\begin{array}{c}\text { Numb / } \\
\text { Detached / } \\
\text { Lack of } \\
\text { energy or } \\
\text { interest }\end{array}$ & $\begin{array}{l}\text { not giving a damn about anything } \\
\text { (a.k.a. the zombification effect). }\end{array}$ \\
\hline $2 / 12 / 2010$ & $\begin{array}{r}\text { Case } \\
\# 1084\end{array}$ & Learn to live with it/deal with it & $\begin{array}{l}\text { Learn to live } \\
\text { with it }\end{array}$ & $\begin{array}{l}\text { Except for the excessive sleepiness, } \\
\text { which could hang around for as long } \\
\text { as you take this med. }\end{array}$ \\
\hline
\end{tabular}




\begin{tabular}{|c|c|c|c|c|}
\hline Date & Case & Memo & Code & Consumer Comment \\
\hline $3 / 10 / 2010$ & $\begin{array}{r}\text { Case } \\
\# 1058\end{array}$ & Finding a doc & $\begin{array}{l}\text { Other } \\
\text { doctor- } \\
\text { related } \\
\text { issues }\end{array}$ & $\begin{array}{l}\text { Re. docs, ask any docs (of any type) } \\
\text { that you trust to give you names of any } \\
\text { pdocs (psychiatrists) they've heard } \\
\text { particularly good stuff about (and/or } \\
\text { deal with bipolar a lot). }\end{array}$ \\
\hline $3 / 10 / 2010$ & $\begin{array}{r}\text { Case } \\
\# 1058\end{array}$ & Finding a doc & $\begin{array}{l}\text { Other } \\
\text { doctor- } \\
\text { related } \\
\text { issues }\end{array}$ & $\begin{array}{l}\text { Last change I had to make, I asked my } \\
\text { primary care doc, therapist, old doc } \\
\text { (she was going to work for the VA), my } \\
\text { husband's doc at the VA, our couples' } \\
\text { therapist at the VA . . I heard the } \\
\text { same name from a couple of people I } \\
\text { really respected, tried that doc \& am } \\
\text { really pleased with him. Of course, if I } \\
\text { hadn't been, it'd have been back out } \\
\text { there again. You have to have } \\
\text { someone you feel is competent \& does } \\
\text { care (within the very limited time they } \\
\text { can give) }\end{array}$ \\
\hline $3 / 10 / 2010$ & $\begin{array}{r}\text { Case } \\
\# 1065\end{array}$ & Med hoarding / disposing & $\begin{array}{c}\text { Making a } \\
\text { plan / Just } \\
\text { in case... }\end{array}$ & $\begin{array}{l}\text { I absolutely horde medication. } \\
\text { Prescriptions, over the counter meds, } \\
\text { herbs, vitamins, supplements, } \\
\text { tinctures, teas, expired, variously } \\
\text { acquired, frequently useful, but some } \\
\text { of which I can't even remember what } \\
\text { they do or how i came to posses them. } \\
\text { And all with this vague concept that I } \\
\text { might need them either for myself or } \\
\text { others, or, on bad days, because i } \\
\text { might at some point decide to } \\
\text { consume all of everything and see } \\
\text { what happens. but mostly i just can't } \\
\text { seem to make myself get rid of these } \\
\text { things. }\end{array}$ \\
\hline $3 / 10 / 2010$ & $\begin{array}{r}\text { Case } \\
\# 1069\end{array}$ & $\begin{array}{l}\text { Afraid of stopping or changing } \\
\text { meds }\end{array}$ & $\begin{array}{l}\text { Looking } \\
\text { back on } \\
\text { it.../Since I } \\
\text { stopped the } \\
\text { med... }\end{array}$ & $\begin{array}{l}\text { afraid to go off meds because of what } \\
\text { I've described elsewhere as the illness } \\
\text { I felt all the time once off them. As for } \\
\text { the headaches, I don't have them } \\
\text { anymore, but I don't know what would } \\
\text { happen if I tried anything new out. As } \\
\text { I've said elsewhere, Zyprexa has } \\
\text { sometimes been a weight gainer for } \\
\text { me, although there are other reasons } \\
\text { why I've put on weight in the past } \\
\text { year...but I can't let go of it because of } \\
\text { the year I lost of my life to all sorts of } \\
\text { other meds. And that started with evil } \\
\text { deathforce Seroquel. }\end{array}$ \\
\hline
\end{tabular}




\begin{tabular}{|c|c|c|c|c|}
\hline Date & Case & Memo & Code & Consumer Comment \\
\hline $3 / 10 / 2010$ & $\begin{array}{r}\text { Case } \\
\# 1070\end{array}$ & $\begin{array}{l}\text { I am not sure where I am putting } \\
\text { these comments that } \\
\text { recommend someone get } \\
\text { regular tests for something: } \\
\text { either "medical tests" (but it's } \\
\text { really not Evidence of Causality) } \\
\text { or "I'm taking control" (because } \\
\text { they are monitoring drug effects) } \\
\text { or something else? }\end{array}$ & $\begin{array}{l}\text { Check blood } \\
\text { levels }\end{array}$ & $\begin{array}{l}\text { Also, if you take Seroquel, it's good to } \\
\text { have a fasting blood sugar check } \\
\text { every few months (a fingerstick at } \\
\text { least } 2 \text { hours after eating or drinking } \\
\text { anything except water.) }\end{array}$ \\
\hline $3 / 10 / 2010$ & $\begin{array}{r}\text { Case } \\
\# 1073\end{array}$ & meds and pregnancy & OTHER & $\begin{array}{l}\text { i'm on lithium and zyprexa. my doctor's } \\
\text { recommendation was to stay on both } \\
\text { throughout the pregnancy, and i'm } \\
\text { going to (i tried to get off the lithium } \\
\text { but it was a disaster). }\end{array}$ \\
\hline $3 / 11 / 2010$ & $\begin{array}{l}\text { Case } \\
\# 135\end{array}$ & $\begin{array}{l}\text { Comment about having to weigh } \\
\text { benefits and costs - though not } \\
\text { making a decision about them. }\end{array}$ & $\begin{array}{l}\text { No or } \\
\text { limited } \\
\text { helpfulness } \\
\text { / It's not my } \\
\text { wonder drug }\end{array}$ & $\begin{array}{l}\text { If you do gain weight (which isn't } \\
\text { guaranteed in the first place), I think } \\
\text { it's important to consider how much } \\
\text { weight you actually gain vs. how well } \\
\text { the antidepressant is helping your } \\
\text { mood. It may still be worth it. }\end{array}$ \\
\hline $3 / 11 / 2010$ & $\begin{array}{l}\text { Case } \\
\# 135\end{array}$ & $\begin{array}{l}\text { Brain zaps on Lexapro (while on } \\
\text { a stable dose) }\end{array}$ & $\begin{array}{l}\text { Zaps / } \\
\text { Tingling }\end{array}$ & $\begin{array}{l}\text { I've been on } 20 \text { mg Lexapro for a year } \\
\text { and a half at this point, perhaps } \\
\text { longer. I've also been on } 200 \text { mg of } \\
\text { generic Wellbutrin ( } 100 \text { mg taken twice } \\
\text { a day) for the better part of the year. } \\
\text { The zaps aren't comparable to the } \\
\text { ones I experienced during Effexor } \\
\text { withdrawal, but they're nonetheless } \\
\text { annoying. (I assume they have more } \\
\text { to do with Lexapro than with } \\
\text { Wellbutrin.) I've been having them for } \\
3 \text { days at this point, but I haven't been } \\
\text { missed a dose of my meds. (OK, I did } \\
\text { forget to take my stupid statin last } \\
\text { night, but that shouldn't be related, } \\
\text { right?) }\end{array}$ \\
\hline $3 / 11 / 2010$ & $\begin{array}{l}\text { Case } \\
\# 135\end{array}$ & $\begin{array}{l}\text { Comment about having to make } \\
\text { an assessment, but no decision } \\
\text { made... }\end{array}$ & $\begin{array}{l}\text { No or } \\
\text { limited } \\
\text { helpfulness } \\
\text { / It's not my } \\
\text { wonder drug }\end{array}$ & $\begin{array}{l}\text { At the end of the trial period, you'll be } \\
\text { able to decide whether the benefits are } \\
\text { worth whatever side effects you might } \\
\text { develop. (There are some lucky } \\
\text { people who don't experience any side } \\
\text { effects to speak of.) }\end{array}$ \\
\hline
\end{tabular}




\begin{tabular}{|c|c|c|c|c|}
\hline Date & Case & Memo & Code & Consumer Comment \\
\hline $3 / 11 / 2010$ & $\begin{array}{l}\text { Case } \\
\# 135\end{array}$ & Learn to live with it & $\begin{array}{l}\text { Learn to live } \\
\text { with it }\end{array}$ & $\begin{array}{l}\text { Do Sexual Side Effects Ever Wear } \\
\text { Off? Posted by: Artemisia Mon } 10 \\
\text { December } 2007 \text { 15:52:53 GMT +0000 } \\
\text { I'm one of those "zero, zilch, nada, } \\
\text { nothing, never" cases. Nothing works } \\
\text { even when I'm not on any meds... }\end{array}$ \\
\hline $3 / 11 / 2010$ & $\begin{array}{l}\text { Case } \\
\# 964\end{array}$ & $\begin{array}{l}\text { Not so much the 'doctor' but the } \\
\text { 'system' is wrong }\end{array}$ & $\begin{array}{l}\text { Doctor won't } \\
\text { listen / We } \\
\text { disagree / I } \\
\text { wasn't } \\
\text { informed }\end{array}$ & $\begin{array}{l}\text { The whole system needs to change. I } \\
\text { think if only I was taught to live with } \\
\text { specific symptoms. Like I have a fast } \\
\text { brain. No doubt about it- it's just fast. } \\
\text { They (medical community) wanted to } \\
\text { slow it down and I downed pills for it. } \\
\text { But I couldn't deal with a slower brain- } \\
\text { that made me more crazy. But no one } \\
\text { saw that. If only I were taught how to } \\
\text { handle it going fast- taught how to } \\
\text { organize my fast thoughts. I had to } \\
\text { learn that stuff the hard way. I self- } \\
\text { taught myself to handle my speech so } \\
\text { its understandable not fast and } \\
\text { jumping around topics. I may be } \\
\text { thinking that fast but I've learned how } \\
\text { to have appropriate conversations. }\end{array}$ \\
\hline $3 / 11 / 2010$ & $\begin{array}{l}\text { Case } \\
\# 964\end{array}$ & Thinking of going back on meds & $\begin{array}{l}\text { Going back } \\
\text { on meds / } \\
\text { Meds may } \\
\text { be the } \\
\text { solution }\end{array}$ & $\begin{array}{l}\text { At times I wonder about going back on } \\
\text { Ritalin but only because my } \\
\text { organizational skills are nil. I can't } \\
\text { organize for the life of me and it } \\
\text { shows- my desk is always a mess as } \\
\text { is my whole therapy area where I treat } \\
\text { the kids. It is a constant struggle for } \\
\text { me to keep it all together. }\end{array}$ \\
\hline $3 / 11 / 2010$ & $\begin{array}{l}\text { Case } \\
\# 964\end{array}$ & $\begin{array}{l}\text { Related to "the system" - but not } \\
\text { jumping through hoops. I coded } \\
\text { this here because if this user } \\
\text { was complaining about "the } \\
\text { hospital" or "the system" I would } \\
\text { code it here. I may need to re- } \\
\text { label this code to reflect any } \\
\text { comments related to 'the } \\
\text { system,' then. }\end{array}$ & $\begin{array}{l}\text { Jumping } \\
\text { through } \\
\text { hoops / } \\
\text { Hospitals }\end{array}$ & $\begin{array}{l}\text { hospitals didn't destroy my life- I still } \\
\text { went to college full time, I still held a } \\
\text { full time job (though it had flexible } \\
\text { hours to allow me the time off } \\
\text { sometimes)...I still had a very full life, } \\
\text { just with tiny interludes to get my head } \\
\text { straight. And to this day even though } \\
\text { I am shifting away from a pure medical } \\
\text { model, if I went nuts for some } \\
\text { reason...I still know that a hospital can } \\
\text { give me reprieve's. }\end{array}$ \\
\hline $3 / 11 / 2010$ & $\begin{array}{l}\text { Case } \\
\# 964\end{array}$ & $\begin{array}{l}\text { Thinking of going back on a } \\
\text { med; prevention }\end{array}$ & $\begin{array}{l}\text { Making a } \\
\text { plan / Just } \\
\text { in case... }\end{array}$ & $\begin{array}{l}\text { So this post is about PREVENTION } \\
\text { and not INTERVENTION. I want to } \\
\text { head off any bad things now before } \\
\text { anything happens and I think going } \\
\text { back on a mood stabilizer may help. }\end{array}$ \\
\hline
\end{tabular}




\begin{tabular}{|c|c|c|c|c|}
\hline Date & Case & Memo & Code & Consumer Comment \\
\hline $3 / 12 / 2010$ & $\begin{array}{l}\text { Case } \\
\# 135\end{array}$ & Helped IBS & $\begin{array}{c}\text { Other Misc } \\
\text { Effects }\end{array}$ & $\begin{array}{l}\text { I've been tentatively diagnosed with } \\
\text { IBS a couple of years ago (I was on } \\
\text { Effexor XR then). I've been oscillating } \\
\text { between constipation and diarrhea. } \\
\text { Switching to Lexapro actually made } \\
\text { my intestinal adventures less bad for a } \\
\text { while, but then they returned. They're } \\
\text { still not as bad as they were on Effexor } \\
\text { XR, though. }\end{array}$ \\
\hline $3 / 14 / 2010$ & $\begin{array}{r}\text { Case } \\
\# 1045\end{array}$ & $\begin{array}{l}\text { I think I've been coding any } \\
\text { "hospital experience" here just to } \\
\text { have them all in the same place. }\end{array}$ & $\begin{array}{l}\text { Jumping } \\
\text { through } \\
\text { hoops / } \\
\text { Hospitals }\end{array}$ & $\begin{array}{l}\text { i remember having one of those } \\
\text { experiences getting locked up (in beth } \\
\text { israel medical center on } 17 \text { th and } 1 \mathrm{st} \text { ) } \\
\text { and i was totally freaking out having a } \\
\text { panic attack thinking i was dying and } \\
\text { the psych doctor in his glasses and } \\
\text { white coat said that he could give a pill } \\
\text { that would help me and i told him to } \\
\text { fuck off, but then a couple hours later } \\
\text { at the request of some friends i gave in } \\
\text { and took it (it was xanax) and a couple } \\
\text { minutes later i felt totally fine. it was } \\
\text { pretty unnerving and quite a relief at } \\
\text { the same time. }\end{array}$ \\
\hline $3 / 14 / 2010$ & $\begin{array}{r}\text { Case } \\
\# 1066\end{array}$ & $\begin{array}{l}\text { I have come to put all comments } \\
\text { related to therapies other than } \\
\text { drugs here - so this now } \\
\text { includes positive comments } \\
\text { about other therapies, negative } \\
\text { comments/ disadvantages / } \\
\text { problems with other therapies, } \\
\text { and suggestions for other } \\
\text { therapies to seek out. }\end{array}$ & $\begin{array}{l}\text { Trying/Tried } \\
\text { alternative } \\
\text { treatment }\end{array}$ & $\begin{array}{l}\text { I want out. I don't want to do it } \\
\text { anymore. I'm sick of the heart-to-heart } \\
\text { chats, the raw disclosure, the drama } \\
\text { therapy, the art therapy, the group } \\
\text { therapy. I want to be left alone, but you } \\
\text { say that and they gently suggest you } \\
\text { write an "action plan" to schedule } \\
\text { "don't hurt self" into your unmonitored } \\
\text { evening. No one is close enough to } \\
\text { help, and those who are close are held } \\
\text { at arm's length because the idea of } \\
\text { being misunderstood by people I care } \\
\text { about so much is too painful to } \\
\text { contemplate experiencing. I think the } \\
\text { only treatment is self acceptance. } \\
\text { Getting there, however, is not always } \\
\text { achieved. What becomes of those } \\
\text { who fail? }\end{array}$ \\
\hline
\end{tabular}




\begin{tabular}{|c|c|c|c|c|}
\hline Date & Case & Memo & Code & Consumer Comment \\
\hline $3 / 14 / 2010$ & $\begin{array}{r}\text { Case } \\
\# 1066\end{array}$ & $\begin{array}{l}\text { Making the decision to go back } \\
\text { on meds }\end{array}$ & $\begin{array}{l}\text { Going back } \\
\text { on meds / } \\
\text { Meds may } \\
\text { be the } \\
\text { solution }\end{array}$ & $\begin{array}{l}\text { For years I thought I could maintain on } \\
\text { my own, but when it became } \\
\text { impossible to do my boring desk job, it } \\
\text { was clear I needed help, and therapy } \\
\text { once a week isn't enough. I have an } \\
\text { appointment soon, thank goodness. I } \\
\text { want to get this ball rolling. I only see } \\
\text { my moods getting more violent as time } \\
\text { goes on. Maybe after I get some } \\
\text { stability, I'll be better able to attend to } \\
\text { the alternative methods that I don't } \\
\text { have the concentration to be diligent } \\
\text { about now. I quit Paxil cold } \\
\text { turkey...I'm sure I can quit again when } \\
\text { the time comes. }\end{array}$ \\
\hline $3 / 30 / 2010$ & $\begin{array}{l}\text { Case } \\
\# 675\end{array}$ & $\begin{array}{l}\text { In response to a person who has } \\
\text { missed } 2 \text { days of Seroquel and } \\
\text { is suddenly very itchy all over. } \\
\text { Jerod proposes the itchiness is } \\
\text { caused by what he explains } \\
\text { here... }\end{array}$ & $\begin{array}{l}\text { Alleviates } \\
\text { side effect } \\
\text { from } \\
\text { another } \\
\text { drug }\end{array}$ & $\begin{array}{l}\text { You've increased the Lamictal since } \\
\text { you've been on Seroquel, right? } \\
\text { Seroquel's antihistamine qualities have } \\
\text { probably been suppressing Lamictal's } \\
\text { itchy side effect. Or Wellbutrin's. In any } \\
\text { event it took care of a side effect from } \\
\text { one or more of them. }\end{array}$ \\
\hline $4 / 2 / 2010$ & $\begin{array}{l}\text { Case } \\
\# 612\end{array}$ & $\begin{array}{l}\text { "Sedating" is difficult to code } \\
\text { because it's a neutral term. }\end{array}$ & $\begin{array}{c}\text { Extreme } \\
\text { sleepiness / } \\
\text { Tired }\end{array}$ & $\begin{array}{l}\text { Seroquel is sedating. There's no way } \\
\text { around that. }\end{array}$ \\
\hline $4 / 4 / 2010$ & $\begin{array}{l}\text { Case } \\
\# 612\end{array}$ & $\begin{array}{l}\text { Not sure the best place to code } \\
\text { this. Kind of saying that the drug } \\
\text { didn't work for them, but may } \\
\text { work for you (hence, YMMV). } \\
\text { But it is an interesting example } \\
\text { of Drugs Only Have Effects that } \\
\text { Some Will Find Helpful and } \\
\text { Other Will Not. }\end{array}$ & YMMV & $\begin{array}{l}\text { Lexapro. I hated the stuff, but the } \\
\text { reason I hated the stuff is the same } \\
\text { reason I'm going to suggest it. It } \\
\text { makes you not care as much. (At least } \\
\text { that's what it did for me.) }\end{array}$ \\
\hline $4 / 4 / 2010$ & $\begin{array}{r}\text { Case } \\
\# 1064\end{array}$ & $\begin{array}{l}\text { Commenting on cognitive } \\
\text { decline over time on meds. } \\
\text { Another example of weighing } \\
\text { benefits and costs, but without } \\
\text { explicitly stating which } \\
\text { outweighs which - it's simply a } \\
\text { difficult trade-off. }\end{array}$ & $\begin{array}{l}\text { No or } \\
\text { limited } \\
\text { helpfulness } \\
\text { / It's not my } \\
\text { wonder drug }\end{array}$ & $\begin{array}{l}\text { When I read what I wrote even } 10 \\
\text { years ago, I know I was smarter then. } \\
\text { But I also know I would not be alive } \\
\text { now had it not been for the drugs. }\end{array}$ \\
\hline
\end{tabular}




\begin{tabular}{|c|c|c|c|l|}
\hline Date & Case & \multicolumn{1}{|c|}{ Memo } & Code & \multicolumn{1}{c|}{ Consumer Comment } \\
\hline $4 / 4 / 2010$ & $\begin{array}{c}\text { Case } \\
\# 1064\end{array}$ & Again, the trade-off. & $\begin{array}{c}\text { No or } \\
\text { limited } \\
\text { helpfulness } \\
\text { / It's not my } \\
\text { wonder drug }\end{array}$ & $\begin{array}{l}\text { While I'm grateful that I am no longer } \\
\text { as mad as I was, I know that I have } \\
\text { lost an edge. While it was dangerous } \\
\text { and painful at times, this edge gave } \\
\text { me the ability to use my madness as a } \\
\text { tool, a weapon and a shield in the on- } \\
\text { going struggles for Truth, Justice, Etc. } \\
\text { I am not as effective as an activist. }\end{array}$ \\
\hline
\end{tabular}




\section{APPENDIX G}

Resolutions to Inter-Coder Disagreements (Decision is in bold)

\begin{tabular}{|c|c|c|c|}
\hline Case & Code & $1^{\text {st }}$ coder & $2^{\text {nd }}$ coder \\
\hline Case \#101 & Evidence of Causality (Any comment) & No & Yes \\
\hline Case \#101 & Gastrointestinal and Urinary & No & Yes \\
\hline Case \#101 & My Diagnosis / Reason for Use (Only for Lex \& Ser) & Yes & No \\
\hline Case \#101 & Other Drug Issues (Any comment) & Yes & No \\
\hline Case \#101 & Other Effects & Yes & No \\
\hline Case \#105 & $\begin{array}{l}\text { Dealing with side effects / Finding optimum } \\
\text { effectiveness (Any comment) }\end{array}$ & Yes & No \\
\hline Case \#108 & Friends and Family (Any comment) & No & Yes \\
\hline Case \#109 & Evidence of Causality (Any comment) & No & Yes \\
\hline Case \#109 & Gastrointestinal and Urinary & Yes & No \\
\hline Case \#109 & Head or Face & Yes & No \\
\hline Case \#109 & Sexual changes & Yes & No \\
\hline Case \#112 & My Doctor (Any comment) & No & Yes \\
\hline Case \#112 & My Drug Experience (Only for Lex \& Ser) & Yes & No \\
\hline Case \#112 & Other Drug Issues (Any comment) & Yes & No \\
\hline Case \#123 & Classification of Effects (Overlap with an Effect) & No & Yes \\
\hline Case \#123 & My Drug Experience (Only for Lex \& Ser) & Yes & No \\
\hline Case \#123 & Other Drug Issues (Any comment) & Yes & No \\
\hline Case \#123 & Other Effects & No & Yes \\
\hline Case \#13 & My Drug Experience (Only for Lex \& Ser) & Yes & No \\
\hline Case \#13 & Other Effects & Yes & No \\
\hline Case \#13 & Theories of Drug Action (Any comment) & No & Yes \\
\hline Case \#134 & Classification of Effects (Overlap with an Effect) & No & Yes \\
\hline Case \#134 & Evidence of Causality (Any comment) & No & Yes \\
\hline Case \#134 & My Doctor (Any comment) & No & Yes \\
\hline Case \#134 & Other Drugs and Drug Combinations (Any comment) & No & Yes \\
\hline Case \#134 & $\begin{array}{l}\text { Weighing Benefits vs Side Effects/Cost (Any } \\
\text { comment) }\end{array}$ & No & Yes \\
\hline Case \#138 & Classification of Effects (Overlap with an Effect) & No & Yes \\
\hline Case \#138 & Evidence of Causality (Any comment) & Yes & No \\
\hline Case \#138 & My Drug Experience (Only for Lex \& Ser) & No & Yes \\
\hline Case \#138 & Theories of Drug Action (Any comment) & No & Yes \\
\hline Case \#139 & My Drug Experience (Only for Lex \& Ser) & No & Yes \\
\hline Case \#139 & Other Drug Issues (Any comment) & No & Yes \\
\hline Case \#139 & Other Drugs and Drug Combinations (Any comment) & Yes & No \\
\hline Case \#139 & Other Effects & Yes & No \\
\hline Case \#139 & $\begin{array}{l}\text { Weighing Benefits vs Side Effects/Cost (Any } \\
\text { comment) }\end{array}$ & Yes & No \\
\hline Case \#151 & $\begin{array}{l}\text { Dealing with side effects / Finding optimum } \\
\text { effectiveness (Any comment) }\end{array}$ & No & Yes \\
\hline Case \#151 & Pharmaceutical Companies / FDA & No & Yes \\
\hline Case \#160 & Classification of Effects (Overlap with an Effect) & No & Yes \\
\hline
\end{tabular}




\begin{tabular}{|c|c|c|c|}
\hline Case & Code & $1^{\text {st }}$ coder & $2^{\text {nd }}$ coder \\
\hline Case \#160 & My Drug Experience (Only for Lex \& Ser) & No & Yes \\
\hline Case \#163 & Classification of Effects (Overlap with an Effect) & Yes & No \\
\hline Case \#163 & Other Effects & Yes & No \\
\hline Case \#164 & $\begin{array}{l}\text { Dealing with side effects / Finding optimum } \\
\text { effectiveness (Any comment) }\end{array}$ & No & Yes \\
\hline Case \#169 & Classification of Effects (Overlap with an Effect) & No & Yes \\
\hline Case \#169 & Evidence of Causality (Any comment) & Yes & No \\
\hline Case \#169 & My Diagnosis / Reason for Use (Only for Lex \& Ser) & No & Yes \\
\hline Case \#169 & My Doctor (Any comment) & Yes & No \\
\hline Case \#169 & Other Drug Issues (Any comment) & No & Yes \\
\hline Case \#174 & My Drug Experience (Only for Lex \& Ser) & Yes & No \\
\hline Case \#174 & Other Drugs and Drug Combinations (Any comment) & No & Yes \\
\hline Case \#174 & $\begin{array}{l}\text { Weighing Benefits vs Side Effects/Cost (Any } \\
\text { comment) }\end{array}$ & Yes & No \\
\hline Case \#176 & $\begin{array}{l}\text { Dealing with side effects / Finding optimum } \\
\text { effectiveness (Any comment) }\end{array}$ & Yes & No \\
\hline Case \#176 & Other Drug Issues (Any comment) & No & Yes \\
\hline Case \#178 & Classification of Effects (Overlap with an Effect) & No & Yes \\
\hline Case \#178 & $\begin{array}{l}\text { Dealing with side effects / Finding optimum } \\
\text { effectiveness (Any comment) }\end{array}$ & No & Yes \\
\hline Case \#179 & Classification of Effects (Overlap with an Effect) & Yes & No \\
\hline Case \#179 & Evidence of Causality (Any comment) & No & Yes \\
\hline Case \#179 & Friends and Family (Any comment) & No & Yes \\
\hline Case \#179 & My Diagnosis / Reason for Use (Only for Lex \& Ser) & Yes & No \\
\hline Case \#179 & My Doctor (Any comment) & No & Yes \\
\hline Case \#180 & Friends and Family (Any comment) & No & Yes \\
\hline Case \#180 & My Diagnosis / Reason for Use (Only for Lex \& Ser) & Yes & No \\
\hline Case \#180 & Other Drug Issues (Any comment) & No & Yes \\
\hline Case \#184 & Classification of Effects (Overlap with an Effect) & No & Yes \\
\hline Case \#184 & Other Drugs and Drug Combinations (Any comment) & No & Yes \\
\hline Case \#185 & Classification of Effects (Overlap with an Effect) & No & Yes \\
\hline Case \#185 & Contraindications (Only for Lex \& Ser) & Yes & No \\
\hline Case \#185 & Theories of Drug Action (Any comment) & No & Yes \\
\hline Case \#187 & Classification of Effects (Overlap with an Effect) & No & Yes \\
\hline Case \#187 & My Doctor (Any comment) & No & Yes \\
\hline Case \#187 & Theories of Drug Action (Any comment) & No & Yes \\
\hline Case \#191 & Evidence of Causality (Any comment) & No & Yes \\
\hline Case \#191 & Muscoloskeletal and Neurological & No & Yes \\
\hline Case \#191 & Other Effects & No & Yes \\
\hline Case \#191 & $\begin{array}{l}\text { Weighing Benefits vs Side Effects/Cost (Any } \\
\text { comment) }\end{array}$ & No & Yes \\
\hline Case \#197 & Support and Advice (Any comment) & No & Yes \\
\hline Case \#215 & $\begin{array}{l}\text { Dealing with side effects / Finding optimum } \\
\text { effectiveness (Any comment) }\end{array}$ & No & Yes \\
\hline Case \#215 & My Diagnosis / Reason for Use (Only for Lex \& Ser) & No & Yes \\
\hline Case \#215 & Other Drug Issues (Any comment) & No & Yes \\
\hline
\end{tabular}




\begin{tabular}{|c|c|c|c|}
\hline Case & Code & $1^{\text {st }}$ coder & $2^{\text {nd }}$ coder \\
\hline Case \#216 & Classification of Effects (Overlap with an Effect) & No & Yes \\
\hline Case \#218 & Other Drug Issues (Any comment) & No & Yes \\
\hline Case \#218 & Other Drugs and Drug Combinations (Any comment) & Yes & No \\
\hline Case \#22 & Classification of Effects (Overlap with an Effect) & No & Yes \\
\hline Case \#22 & Gastrointestinal and Urinary & Yes & No \\
\hline Case \#239 & Classification of Effects (Overlap with an Effect) & No & Yes \\
\hline Case \#239 & Evidence of Causality (Any comment) & Yes & No \\
\hline Case \#239 & Mental or mood changes & No & Yes \\
\hline Case \#239 & My Doctor (Any comment) & No & Yes \\
\hline Case \#243 & $\begin{array}{l}\text { Dealing with side effects / Finding optimum } \\
\text { effectiveness (Any comment) }\end{array}$ & Yes & No \\
\hline Case \#243 & My Drug Experience (Only for Lex \& Ser) & No & Yes \\
\hline Case \#243 & Other Drugs and Drug Combinations (Any comment) & No & Yes \\
\hline Case \#243 & $\begin{array}{l}\text { Weighing Benefits vs Side Effects/Cost (Any } \\
\text { comment) }\end{array}$ & Yes & No \\
\hline Case \#244 & My Drug Experience (Only for Lex \& Ser) & No & Yes \\
\hline Case \#244 & Other Drugs and Drug Combinations (Any comment) & Yes & No \\
\hline Case \#244 & Other Effects & No & Yes \\
\hline Case \#244 & $\begin{array}{l}\text { Weighing Benefits vs Side Effects/Cost (Any } \\
\text { comment) }\end{array}$ & Yes & No \\
\hline Case \#252 & Classification of Effects (Overlap with an Effect) & No & Yes \\
\hline Case \#252 & Mental or mood changes & Yes & No \\
\hline Case \#253 & Classification of Effects (Overlap with an Effect) & No & Yes \\
\hline Case \#253 & Mental or mood changes & Yes & No \\
\hline Case \#253 & Support and Advice (Any comment) & No & Yes \\
\hline Case \#253 & $\begin{array}{l}\text { Weighing Benefits vs Side Effects/Cost (Any } \\
\text { comment) }\end{array}$ & Yes & No \\
\hline Case \#256 & My Doctor (Any comment) & No & Yes \\
\hline Case \#256 & Other Drug Issues (Any comment) & No & Yes \\
\hline Case \#259 & $\begin{array}{l}\text { Dealing with side effects / Finding optimum } \\
\text { effectiveness (Any comment) }\end{array}$ & No & Yes \\
\hline Case \#259 & My Drug Experience (Only for Lex \& Ser) & No & Yes \\
\hline Case \#259 & Other Effects & No & Yes \\
\hline Case \#27 & $\begin{array}{l}\text { Weighing Benefits vs Side Effects/Cost (Any } \\
\text { comment) }\end{array}$ & Yes & No \\
\hline Case \#270 & Mental or mood changes & Yes & No \\
\hline Case \#270 & My Diagnosis / Reason for Use (Only for Lex \& Ser) & Yes & No \\
\hline Case \#270 & My Drug Experience (Only for Lex \& Ser) & Yes & No \\
\hline Case \#270 & Other Drug Issues (Any comment) & No & Yes \\
\hline Case \#270 & Other Drugs and Drug Combinations (Any comment) & No & Yes \\
\hline Case \#271 & Classification of Effects (Overlap with an Effect) & No & Yes \\
\hline Case \#271 & $\begin{array}{l}\text { Dealing with side effects / Finding optimum } \\
\text { effectiveness (Any comment) }\end{array}$ & No & Yes \\
\hline Case \#271 & Other Drug Issues (Any comment) & No & Yes \\
\hline Case \#277 & Gastrointestinal and Urinary & Yes & No \\
\hline Case \#277 & Other Effects & No & Yes \\
\hline
\end{tabular}




\begin{tabular}{|c|c|c|c|}
\hline Case & Code & $1^{\text {st }}$ coder & $2^{\text {nd }}$ coder \\
\hline Case \#279 & Classification of Effects (Overlap with an Effect) & Yes & No \\
\hline Case \#279 & $\begin{array}{l}\text { Weighing Benefits vs Side Effects/Cost (Any } \\
\text { comment) }\end{array}$ & Yes & No \\
\hline Case \#288 & Classification of Effects (Overlap with an Effect) & No & Yes \\
\hline Case \#288 & My Drug Experience (Only for Lex \& Ser) & Yes & No \\
\hline Case \#288 & Other Drugs and Drug Combinations (Any comment) & No & Yes \\
\hline Case \#288 & $\begin{array}{l}\text { Weighing Benefits vs Side Effects/Cost (Any } \\
\text { comment) }\end{array}$ & No & Yes \\
\hline Case \#292 & Classification of Effects (Overlap with an Effect) & No & Yes \\
\hline Case \#292 & Evidence of Causality (Any comment) & Yes & No \\
\hline Case \#292 & My Doctor (Any comment) & No & Yes \\
\hline Case \#292 & Other Drug Issues (Any comment) & No & Yes \\
\hline Case \#293 & Friends and Family (Any comment) & No & Yes \\
\hline Case \#293 & Other Drugs and Drug Combinations (Any comment) & No & Yes \\
\hline Case \#293 & $\begin{array}{l}\text { Weighing Benefits vs Side Effects/Cost (Any } \\
\text { comment) }\end{array}$ & No & Yes \\
\hline Case \#297 & My Diagnosis / Reason for Use (Only for Lex \& Ser) & No & Yes \\
\hline Case \#299 & Classification of Effects (Overlap with an Effect) & No & Yes \\
\hline Case \#299 & Support and Advice (Any comment) & No & Yes \\
\hline Case \#303 & My Drug Experience (Only for Lex \& Ser) & No & Yes \\
\hline Case \#303 & Other Drug Issues (Any comment) & Yes & No \\
\hline Case \#303 & Other Effects & No & Yes \\
\hline Case \#307 & Other Effects & No & Yes \\
\hline Case \#308 & $\begin{array}{l}\text { Dealing with side effects / Finding optimum } \\
\text { effectiveness (Any comment) }\end{array}$ & No & Yes \\
\hline Case \#313 & $\begin{array}{l}\text { Dealing with side effects / Finding optimum } \\
\text { effectiveness (Any comment) }\end{array}$ & No & Yes \\
\hline Case \#313 & Other Drugs and Drug Combinations (Any comment) & Yes & No \\
\hline Case \#313 & Other Effects & No & Yes \\
\hline Case \#315 & $\begin{array}{l}\text { Dealing with side effects / Finding optimum } \\
\text { effectiveness (Any comment) }\end{array}$ & No & Yes \\
\hline Case \#315 & Other Drug Issues (Any comment) & Yes & No \\
\hline Case \#315 & Other Effects & Yes & No \\
\hline Case \#334 & Classification of Effects (Overlap with an Effect) & No & Yes \\
\hline Case \#34 & Muscoloskeletal and Neurological & No & Yes \\
\hline Case \#34 & Other Drug Issues (Any comment) & No & Yes \\
\hline Case \#34 & Other Drugs and Drug Combinations (Any comment) & Yes & No \\
\hline Case \#34 & Support and Advice (Any comment) & No & Yes \\
\hline Case \#342 & Classification of Effects (Overlap with an Effect) & No & Yes \\
\hline Case \#346 & Sleep changes & Yes & No \\
\hline Case \#346 & $\begin{array}{l}\text { Weighing Benefits vs Side Effects/Cost (Any } \\
\text { comment) }\end{array}$ & Yes & No \\
\hline Case \#352 & Classification of Effects (Overlap with an Effect) & No & Yes \\
\hline Case \#352 & Other Drugs and Drug Combinations (Any comment) & No & Yes \\
\hline Case \#352 & Other Effects & No & Yes \\
\hline Case \#352 & Support and Advice (Any comment) & No & Yes \\
\hline
\end{tabular}




\begin{tabular}{|c|c|c|c|}
\hline Case & Code & $1^{\text {st }}$ coder & $2^{\text {nd }}$ coder \\
\hline Case \#357 & Classification of Effects (Overlap with an Effect) & No & Yes \\
\hline Case \#357 & Mental or mood changes & Yes & No \\
\hline Case \#357 & My Diagnosis / Reason for Use (Only for Lex \& Ser) & No & Yes \\
\hline Case \#357 & My Drug Experience (Only for Lex \& Ser) & No & Yes \\
\hline Case \#357 & $\begin{array}{l}\text { Weighing Benefits vs Side Effects/Cost (Any } \\
\text { comment) }\end{array}$ & No & Yes \\
\hline Case \#362 & Classification of Effects (Overlap with an Effect) & No & Yes \\
\hline Case \#362 & My Doctor (Any comment) & No & Yes \\
\hline Case \#362 & Other Drug Issues (Any comment) & Yes & No \\
\hline Case \#363 & Classification of Effects (Overlap with an Effect) & No & Yes \\
\hline Case \#363 & Head or Face & Yes & No \\
\hline Case \#363 & Other Drug Issues (Any comment) & No & Yes \\
\hline Case \#363 & Other Effects & No & Yes \\
\hline Case \#363 & $\begin{array}{l}\text { Weighing Benefits vs Side Effects/Cost (Any } \\
\text { comment) }\end{array}$ & Yes & No \\
\hline Case \#364 & Classification of Effects (Overlap with an Effect) & No & Yes \\
\hline Case \#364 & Friends and Family (Any comment) & No & Yes \\
\hline Case \#364 & Other Drug Issues (Any comment) & No & Yes \\
\hline Case \#373 & Friends and Family (Any comment) & No & Yes \\
\hline Case \#374 & Classification of Effects (Overlap with an Effect) & No & Yes \\
\hline Case \#374 & $\begin{array}{l}\text { Dealing with side effects / Finding optimum } \\
\text { effectiveness (Any comment) }\end{array}$ & No & Yes \\
\hline Case \#374 & Other Effects & No & Yes \\
\hline Case \#377 & My Doctor (Any comment) & No & Yes \\
\hline Case \#385 & Finances / The System (Any comment) & Yes & No \\
\hline Case \#385 & Theories of Drug Action (Any comment) & No & Yes \\
\hline Case \#388 & Classification of Effects (Overlap with an Effect) & No & Yes \\
\hline Case \#390 & Classification of Effects (Overlap with an Effect) & No & Yes \\
\hline Case \#393 & Evidence of Causality (Any comment) & No & Yes \\
\hline Case \#393 & Other Effects & No & Yes \\
\hline Case \#393 & Sleep changes & Yes & No \\
\hline Case \#393 & Support and Advice (Any comment) & No & Yes \\
\hline Case \#394 & Classification of Effects (Overlap with an Effect) & No & Yes \\
\hline Case \#396 & Friends and Family (Any comment) & No & Yes \\
\hline Case \#396 & Other Effects & Yes & No \\
\hline Case \#397 & Other Drug Issues (Any comment) & Yes & No \\
\hline Case \#397 & $\begin{array}{l}\text { Weighing Benefits vs Side Effects/Cost (Any } \\
\text { comment) }\end{array}$ & No & Yes \\
\hline Case \#400 & My Doctor (Any comment) & No & Yes \\
\hline Case \#400 & Other Drugs and Drug Combinations (Any comment) & No & Yes \\
\hline Case \#400 & $\begin{array}{l}\text { Weighing Benefits vs Side Effects/Cost (Any } \\
\text { comment) }\end{array}$ & No & Yes \\
\hline Case \#401 & Classification of Effects (Overlap with an Effect) & No & Yes \\
\hline Case \#401 & My Drug Experience (Only for Lex \& Ser) & No & Yes \\
\hline Case \#401 & $\begin{array}{l}\text { Weighing Benefits vs Side Effects/Cost (Any } \\
\text { comment) }\end{array}$ & Yes & No \\
\hline
\end{tabular}




\begin{tabular}{|c|c|c|c|}
\hline Case & Code & $1^{\text {st }}$ coder & $2^{\text {nd }}$ coder \\
\hline Case \#413 & Other Effects & No & Yes \\
\hline Case \#413 & Sexual changes & Yes & No \\
\hline Case \#415 & Classification of Effects (Overlap with an Effect) & No & Yes \\
\hline Case \#416 & Classification of Effects (Overlap with an Effect) & No & Yes \\
\hline Case \#416 & My Doctor (Any comment) & No & Yes \\
\hline Case \#43 & Other Drug Issues (Any comment) & No & Yes \\
\hline Case \#43 & Other Drugs and Drug Combinations (Any comment) & Yes & No \\
\hline Case \#43 & Other Effects & Yes & No \\
\hline Case \#436 & Classification of Effects (Overlap with an Effect) & No & Yes \\
\hline Case \#445 & Classification of Effects (Overlap with an Effect) & No & Yes \\
\hline Case \#447 & Friends and Family (Any comment) & No & Yes \\
\hline Case \#59 & Other Drug Issues (Any comment) & Yes & No \\
\hline Case \#6 & Evidence of Causality (Any comment) & No & Yes \\
\hline Case \#7 & Other Drug Issues (Any comment) & No & Yes \\
\hline Case \#7 & Support and Advice (Any comment) & No & Yes \\
\hline Case \#80 & $\begin{array}{l}\text { Dealing with side effects / Finding optimum } \\
\text { effectiveness (Any comment) }\end{array}$ & Yes & No \\
\hline Case \#80 & My Doctor (Any comment) & No & Yes \\
\hline Case \#80 & Nose Throat Chest & No & Yes \\
\hline Case \#80 & Skin & No & Yes \\
\hline Case \#80 & Sleep changes & No & Yes \\
\hline Case \#83 & Muscoloskeletal and Neurological & Yes & No \\
\hline Case \#83 & Other Effects & No & Yes \\
\hline Case \#87 & My Doctor (Any comment) & No & Yes \\
\hline Case \#87 & Other Drugs and Drug Combinations (Any comment) & Yes & No \\
\hline Case \#88 & Evidence of Causality (Any comment) & No & Yes \\
\hline Case \#89 & My Drug Experience (Only for Lex \& Ser) & Yes & No \\
\hline Case \#91 & Other Drug Issues (Any comment) & No & Yes \\
\hline Case \#91 & $\begin{array}{l}\text { Weighing Benefits vs Side Effects/Cost (Any } \\
\text { comment) }\end{array}$ & Yes & No \\
\hline Case \#99 & $\begin{array}{l}\text { Dealing with side effects / Finding optimum } \\
\text { effectiveness (Any comment) }\end{array}$ & Yes & No \\
\hline Case \#99 & My Drug Experience (Only for Lex \& Ser) & Yes & No \\
\hline Case \#99 & Support and Advice (Any comment) & No & Yes \\
\hline
\end{tabular}


Part II of Inter-Coder Agreement Resolutions

\begin{tabular}{|c|c|c|c|}
\hline Case & Code & $1^{\text {st }}$ coder & $2^{\text {nd }}$ coder \\
\hline Case \#1001 & Evidence of Causality (Any comment) & Yes & No \\
\hline Case \#1001 & Other Drug Issues (Any comment) & No & Yes \\
\hline Case \#1002 & Citations and Links (Any comment) & Yes & No \\
\hline Case \#1002 & Evidence of Causality (Any comment) & No & Yes \\
\hline Case \#1002 & Support and Advice (Any comment) & No & Yes \\
\hline Case \#1003 & Assessing the Overall Experience & Yes & No \\
\hline Case \#1003 & Evidence of Causality (Any comment) & No & Yes \\
\hline Case \#1005 & Evidence of Causality (Any comment) & Yes & No \\
\hline Case \#1005 & My Diagnosis / Reason for Use (Only for Lex \& Ser) & No & Yes \\
\hline Case \#1005 & Pharmaceutical Companies / FDA & Yes & No \\
\hline Case \#1014 & Citations and Links (Any comment) & No & Yes \\
\hline Case \#1014 & Evidence of Causality (Any comment) & No & Yes \\
\hline Case \#1020 & Assessing the Overall Experience & Yes & No \\
\hline Case \#1020 & Evidence of Causality (Any comment) & No & Yes \\
\hline Case \#1020 & Friends and Family (Any comment) & No & Yes \\
\hline Case \#1020 & Support and Advice (Any comment) & No & Yes \\
\hline Case \#1021 & Other Drug Issues (Any comment) & Yes & No \\
\hline Case \#1021 & Support and Advice (Any comment) & No & Yes \\
\hline Case \#1028 & Citations and Links (Any comment) & No & Yes \\
\hline Case \#1028 & Contraindications (Only for Lex \& Ser) & Yes & No \\
\hline Case \#1028 & Pharmaceutical Companies / FDA & Yes & No \\
\hline Case \#1028 & Support and Advice (Any comment) & No & Yes \\
\hline Case \#1031 & Support and Advice (Any comment) & No & Yes \\
\hline Case \#1037 & Assessing the Overall Experience & Yes & No \\
\hline Case \#1037 & Evidence of Causality (Any comment) & No & Yes \\
\hline Case \#1037 & Theories of Drug Action (Any comment) & No & Yes \\
\hline Case \#1043 & Classification of Effects (Overlap with an Effect) & No & Yes \\
\hline Case \#1047 & My Doctor (Any comment) & No & Yes \\
\hline Case \#1056 & Citations and Links (Any comment) & No & Yes \\
\hline Case \#1056 & $\begin{array}{l}\text { Dealing with side effects / Finding optimum } \\
\text { effectiveness (Any comment) }\end{array}$ & No & Yes \\
\hline Case \#1056 & Friends and Family (Any comment) & Yes & No \\
\hline Case \#1056 & Other Drug Issues (Any comment) & Yes & No \\
\hline Case \#1060 & Citations and Links (Any comment) & No & Yes \\
\hline Case \#1060 & Evidence of Causality (Any comment) & No & Yes \\
\hline Case \#1060 & My Doctor (Any comment) & Yes & No \\
\hline
\end{tabular}




\begin{tabular}{|c|c|c|c|}
\hline Case & Code & $1^{\text {st }}$ coder & $2^{\text {nd }}$ coder \\
\hline Case \#1060 & Support and Advice (Any comment) & No & Yes \\
\hline Case \#1072 & Other Drug Issues (Any comment) & Yes & No \\
\hline Case \#1072 & Support and Advice (Any comment) & No & Yes \\
\hline Case \#1079 & Muscoloskeletal and Neurological & No & Yes \\
\hline Case \#1079 & Other Drug Issues (Any comment) & No & Yes \\
\hline Case \#1079 & Other Effects & No & Yes \\
\hline Case \#1079 & Support and Advice (Any comment) & No & Yes \\
\hline Case $\# 456$ & Friends and Family (Any comment) & No & Yes \\
\hline Case \#456 & Other Effects & Yes & No \\
\hline Case \#470 & My Diagnosis / Reason for Use (Only for Lex \& Ser) & No & Yes \\
\hline Case $\# 470$ & Other Effects & Yes & No \\
\hline Case $\# 472$ & Classification of Effects (Overlap with an Effect) & Yes & No \\
\hline Case $\# 472$ & Friends and Family (Any comment) & No & Yes \\
\hline Case $\# 472$ & My Diagnosis / Reason for Use (Only for Lex \& Ser) & Yes & No \\
\hline Case $\# 472$ & Other Drug Issues (Any comment) & No & Yes \\
\hline Case $\# 487$ & Support and Advice (Any comment) & No & Yes \\
\hline Case \#511 & Assessing the Overall Experience & Yes & No \\
\hline Case \#511 & $\begin{array}{l}\text { Dealing with side effects / Finding optimum } \\
\text { effectiveness (Any comment) }\end{array}$ & No & Yes \\
\hline Case \#511 & Mental or mood changes & Yes & No \\
\hline Case \#511 & Nose Throat Chest & No & Yes \\
\hline Case \#516 & $\begin{array}{l}\text { Dealing with side effects / Finding optimum } \\
\text { effectiveness (Any comment) }\end{array}$ & Yes & No \\
\hline Case \#516 & Other Drug Issues (Any comment) & No & Yes \\
\hline Case \#519 & Other Drug Issues (Any comment) & No & Yes \\
\hline Case \#533 & My Doctor (Any comment) & No & Yes \\
\hline Case \#533 & Other Drugs and Drug Combinations (Any comment) & No & Yes \\
\hline Case \#537 & Assessing the Overall Experience & No & Yes \\
\hline Case \#537 & Discontinuing / Missed Doses (Only for Lex \& Ser) & No & Yes \\
\hline Case \#537 & Muscoloskeletal and Neurological & Yes & No \\
\hline Case \#537 & My Diagnosis / Reason for Use (Only for Lex \& Ser) & Yes & No \\
\hline Case \#537 & Other Effects & No & Yes \\
\hline Case $\# 545$ & Head or Face & Yes & No \\
\hline Case $\# 545$ & Other Effects & No & Yes \\
\hline Case \#547 & Assessing the Overall Experience & Yes & No \\
\hline Case \#547 & Evidence of Causality (Any comment) & No & Yes \\
\hline Case \#547 & Other Drug Issues (Any comment) & Yes & No \\
\hline Case \#548 & Other Effects & Yes & No \\
\hline
\end{tabular}




\begin{tabular}{|c|c|c|c|}
\hline Case & Code & $1^{\text {st }}$ coder & $2^{\text {nd }}$ coder \\
\hline Case \#548 & Skin & No & Yes \\
\hline Case \#554 & Other Drug Issues (Any comment) & No & Yes \\
\hline Case \#555 & $\begin{array}{l}\text { Dealing with side effects / Finding optimum } \\
\text { effectiveness (Any comment) }\end{array}$ & No & Yes \\
\hline Case \#555 & Other Drugs and Drug Combinations (Any comment) & No & Yes \\
\hline Case \#560 & Mental or mood changes & No & Yes \\
\hline Case \#560 & Other Drug Issues (Any comment) & Yes & No \\
\hline Case $\# 560$ & Other Drugs and Drug Combinations (Any comment) & No & Yes \\
\hline Case \#565 & Classification of Effects (Overlap with an Effect) & No & Yes \\
\hline Case \#565 & $\begin{array}{l}\text { Dealing with side effects / Finding optimum } \\
\text { effectiveness (Any comment) }\end{array}$ & Yes & No \\
\hline Case \#567 & Other Drug Issues (Any comment) & Yes & No \\
\hline Case \#573 & Mental or mood changes & Yes & No \\
\hline Case \#573 & Muscoloskeletal and Neurological & No & Yes \\
\hline Case \#581 & Assessing the Overall Experience & No & Yes \\
\hline Case \#586 & Appetite and Weight & No & Yes \\
\hline Case \#586 & Other Effects & Yes & No \\
\hline Case \#591 & Friends and Family (Any comment) & No & Yes \\
\hline Case \#591 & Muscoloskeletal and Neurological & No & Yes \\
\hline Case \#591 & Support and Advice (Any comment) & No & Yes \\
\hline Case \#599 & Other Drugs and Drug Combinations (Any comment) & No & Yes \\
\hline Case \#599 & Other Effects & No & Yes \\
\hline Case \#599 & Support and Advice (Any comment) & No & Yes \\
\hline Case \#604 & Assessing the Overall Experience & Yes & No \\
\hline Case \#604 & Other Effects & No & Yes \\
\hline Case \#613 & Citations and Links (Any comment) & No & Yes \\
\hline Case \#613 & Pharmaceutical Companies / FDA & Yes & No \\
\hline Case \#613 & Skin & Yes & No \\
\hline Case \#617 & Appetite and Weight & Yes & No \\
\hline Case \#617 & Citations and Links (Any comment) & Yes & No \\
\hline Case \#617 & Classification of Effects (Overlap with an Effect) & Yes & No \\
\hline Case \#617 & Head or Face & Yes & No \\
\hline Case \#617 & Other Drug Issues (Any comment) & Yes & No \\
\hline Case \#617 & Other Drugs and Drug Combinations (Any comment) & No & Yes \\
\hline Case \#617 & Skin & Yes & No \\
\hline Case \#617 & Sleep changes & Yes & No \\
\hline Case \#621 & Evidence of Causality (Any comment) & No & Yes \\
\hline Case \#621 & Other Effects & No & Yes \\
\hline
\end{tabular}




\begin{tabular}{|c|c|c|c|}
\hline Case & Code & $1^{\text {st }}$ coder & $2^{\text {nd }}$ coder \\
\hline Case \#626 & Assessing the Overall Experience & Yes & No \\
\hline Case \#626 & $\begin{array}{l}\text { Dealing with side effects / Finding optimum } \\
\text { effectiveness (Any comment) }\end{array}$ & No & Yes \\
\hline Case \#626 & Support and Advice (Any comment) & No & Yes \\
\hline Case \#629 & Appetite and Weight & No & Yes \\
\hline Case \#629 & Evidence of Causality (Any comment) & No & Yes \\
\hline Case \#629 & My Doctor (Any comment) & No & Yes \\
\hline Case \#629 & Other Drug Issues (Any comment) & No & Yes \\
\hline Case \#642 & Assessing the Overall Experience & Yes & No \\
\hline Case \#642 & Discontinuing / Missed Doses (Only for Lex \& Ser) & Yes & No \\
\hline Case \#642 & Evidence of Causality (Any comment) & Yes & No \\
\hline Case \#642 & Mental or mood changes & No & Yes \\
\hline Case \#642 & Other Effects & No & Yes \\
\hline Case \#642 & Support and Advice (Any comment) & No & Yes \\
\hline Case \#643 & Sleep changes & No & Yes \\
\hline Case \#646 & Evidence of Causality (Any comment) & Yes & No \\
\hline Case \#646 & My Diagnosis / Reason for Use (Only for Lex \& Ser) & No & Yes \\
\hline Case \#646 & Pharmaceutical Companies / FDA & Yes & No \\
\hline Case \#646 & Support and Advice (Any comment) & No & Yes \\
\hline Case \#650 & Classification of Effects (Overlap with an Effect) & No & Yes \\
\hline Case \#650 & Discontinuing / Missed Doses (Only for Lex \& Ser) & Yes & No \\
\hline Case \#650 & My Diagnosis / Reason for Use (Only for Lex \& Ser) & No & Yes \\
\hline Case \#650 & Other Effects & Yes & No \\
\hline Case \#654 & Head or Face & Yes & No \\
\hline Case \#654 & Other Drugs and Drug Combinations (Any comment) & No & Yes \\
\hline Case \#654 & Support and Advice (Any comment) & No & Yes \\
\hline Case \#655 & Assessing the Overall Experience & Yes & No \\
\hline Case \#655 & Classification of Effects (Overlap with an Effect) & No & Yes \\
\hline Case \#655 & Discontinuing / Missed Doses (Only for Lex \& Ser) & Yes & No \\
\hline Case \#655 & My Diagnosis / Reason for Use (Only for Lex \& Ser) & No & Yes \\
\hline Case \#675 & Appetite and Weight & No & Yes \\
\hline Case \#675 & Assessing the Overall Experience & Yes & No \\
\hline Case \#675 & Classification of Effects (Overlap with an Effect) & Yes & No \\
\hline Case \#675 & Mental or mood changes & Yes & No \\
\hline Case \#675 & Muscoloskeletal and Neurological & No & Yes \\
\hline Case \#675 & Support and Advice (Any comment) & No & Yes \\
\hline Case \#681 & Classification of Effects (Overlap with an Effect) & No & Yes \\
\hline
\end{tabular}




\begin{tabular}{|c|c|c|c|}
\hline Case & Code & $1^{\text {st }}$ coder & $2^{\text {nd }}$ coder \\
\hline Case \#681 & Mental or mood changes & No & Yes \\
\hline Case \#683 & $\begin{array}{l}\text { Dealing with side effects / Finding optimum } \\
\text { effectiveness (Any comment) }\end{array}$ & No & Yes \\
\hline Case \#683 & My Doctor (Any comment) & No & Yes \\
\hline Case \#683 & Other Drug Issues (Any comment) & Yes & No \\
\hline Case \#687 & Assessing the Overall Experience & Yes & No \\
\hline Case \#687 & Evidence of Causality (Any comment) & No & Yes \\
\hline Case \#687 & My Diagnosis / Reason for Use (Only for Lex \& Ser) & Yes & No \\
\hline Case \#695 & Classification of Effects (Overlap with an Effect) & Yes & No \\
\hline Case $\# 695$ & $\begin{array}{l}\text { Dealing with side effects / Finding optimum } \\
\text { effectiveness (Any comment) }\end{array}$ & No & Yes \\
\hline Case \#696 & Contraindications (Only for Lex \& Ser) & No & Yes \\
\hline Case \#701 & Appetite and Weight & No & Yes \\
\hline Case \#701 & Other Drugs and Drug Combinations (Any comment) & No & Yes \\
\hline Case \#701 & Sleep changes & No & Yes \\
\hline Case \#704 & Other Drug Issues (Any comment) & Yes & No \\
\hline Case \#704 & Support and Advice (Any comment) & No & Yes \\
\hline Case \#716 & $\begin{array}{l}\text { Dealing with side effects / Finding optimum } \\
\text { effectiveness (Any comment) }\end{array}$ & No & Yes \\
\hline Case \#716 & Pharmaceutical Companies / FDA & Yes & No \\
\hline Case \#718 & Evidence of Causality (Any comment) & No & Yes \\
\hline Case \#719 & Evidence of Causality (Any comment) & Yes & No \\
\hline Case \#719 & Friends and Family (Any comment) & Yes & No \\
\hline Case \#719 & Other Effects & No & Yes \\
\hline Case $\# 720$ & Assessing the Overall Experience & Yes & No \\
\hline Case \#720 & My Diagnosis / Reason for Use (Only for Lex \& Ser) & Yes & No \\
\hline Case \#720 & My Doctor (Any comment) & No & Yes \\
\hline Case \#723 & $\begin{array}{l}\text { Dealing with side effects / Finding optimum } \\
\text { effectiveness (Any comment) }\end{array}$ & No & Yes \\
\hline Case $\# 723$ & Other Drug Issues (Any comment) & No & Yes \\
\hline Case \#725 & Mental or mood changes & Yes & No \\
\hline Case $\# 725$ & Other Effects & No & Yes \\
\hline Case \#747 & Assessing the Overall Experience & No & Yes \\
\hline Case \#747 & Classification of Effects (Overlap with an Effect) & Yes & No \\
\hline Case \#747 & Discontinuing / Missed Doses (Only for Lex \& Ser) & No & Yes \\
\hline Case \#747 & Muscoloskeletal and Neurological & No & Yes \\
\hline Case \#747 & Other Drug Issues (Any comment) & Yes & No \\
\hline Case \#752 & $\begin{array}{l}\text { Dealing with side effects / Finding optimum } \\
\text { effectiveness (Any comment) }\end{array}$ & No & Yes \\
\hline
\end{tabular}




\begin{tabular}{|c|c|c|c|}
\hline Case & Code & $1^{\text {st }}$ coder & $2^{\text {nd }}$ coder \\
\hline Case \#761 & Friends and Family (Any comment) & No & Yes \\
\hline Case \#761 & My Diagnosis / Reason for Use (Only for Lex \& Ser) & No & Yes \\
\hline Case \#761 & Other Drugs and Drug Combinations (Any comment) & No & Yes \\
\hline Case \#772 & My Diagnosis / Reason for Use (Only for Lex \& Ser) & Yes & No \\
\hline Case \#772 & My Doctor (Any comment) & No & Yes \\
\hline Case \#772 & Other Drug Issues (Any comment) & Yes & No \\
\hline Case \#773 & Other Effects & Yes & No \\
\hline Case \#777 & $\begin{array}{l}\text { Dealing with side effects / Finding optimum } \\
\text { effectiveness (Any comment) }\end{array}$ & No & Yes \\
\hline Case \#777 & Head or Face & No & Yes \\
\hline Case \#777 & Nose Throat Chest & No & Yes \\
\hline Case \#777 & Other Drug Issues (Any comment) & No & Yes \\
\hline Case \#779 & Appetite and Weight & No & Yes \\
\hline Case \#779 & Discontinuing / Missed Doses (Only for Lex \& Ser) & No & Yes \\
\hline Case \#779 & Evidence of Causality (Any comment) & Yes & No \\
\hline Case \#779 & Friends and Family (Any comment) & No & Yes \\
\hline Case \#779 & My Doctor (Any comment) & No & Yes \\
\hline Case \#779 & Other Drug Issues (Any comment) & Yes & No \\
\hline Case \#780 & Classification of Effects (Overlap with an Effect) & No & Yes \\
\hline Case \#780 & $\begin{array}{l}\text { Dealing with side effects / Finding optimum } \\
\text { effectiveness (Any comment) }\end{array}$ & Yes & No \\
\hline Case \#784 & Assessing the Overall Experience & Yes & No \\
\hline Case \#784 & My Doctor (Any comment) & No & Yes \\
\hline Case \#784 & Other Drug Issues (Any comment) & No & Yes \\
\hline Case \#786 & Assessing the Overall Experience & No & Yes \\
\hline Case \#786 & Friends and Family (Any comment) & No & Yes \\
\hline Case \#786 & My Doctor (Any comment) & No & Yes \\
\hline Case \#786 & Support and Advice (Any comment) & Yes & No \\
\hline Case \#799 & Evidence of Causality (Any comment) & No & Yes \\
\hline Case \#799 & My Doctor (Any comment) & No & Yes \\
\hline Case \#799 & Other Effects & Yes & No \\
\hline Case \#812 & My Diagnosis / Reason for Use (Only for Lex \& Ser) & Yes & No \\
\hline Case $\# 814$ & Mental or mood changes & No & Yes \\
\hline Case \#828 & Other Drug Issues (Any comment) & No & Yes \\
\hline Case \#832 & Classification of Effects (Overlap with an Effect) & No & Yes \\
\hline Case \#832 & Discontinuing / Missed Doses (Only for Lex \& Ser) & No & Yes \\
\hline Case \#832 & My Diagnosis / Reason for Use (Only for Lex \& Ser) & Yes & No \\
\hline Case \#832 & Other Drug Issues (Any comment) & Yes & No \\
\hline
\end{tabular}




\begin{tabular}{|c|c|c|c|}
\hline Case & Code & $1^{\text {st }}$ coder & $2^{\text {nd }}$ coder \\
\hline Case \#832 & Other Drugs and Drug Combinations (Any comment) & No & Yes \\
\hline Case \#832 & Other Effects & Yes & No \\
\hline Case \#854 & Assessing the Overall Experience & No & Yes \\
\hline Case \#854 & Classification of Effects (Overlap with an Effect) & Yes & No \\
\hline Case \#854 & $\begin{array}{l}\text { Dealing with side effects / Finding optimum } \\
\text { effectiveness (Any comment) }\end{array}$ & No & Yes \\
\hline Case \#854 & Discontinuing / Missed Doses (Only for Lex \& Ser) & No & Yes \\
\hline Case \#854 & Lab Tests & Yes & No \\
\hline Case \#855 & $\begin{array}{l}\text { Dealing with side effects / Finding optimum } \\
\text { effectiveness (Any comment) }\end{array}$ & No & Yes \\
\hline Case \#855 & Friends and Family (Any comment) & No & Yes \\
\hline Case \#855 & Head or Face & No & Yes \\
\hline Case \#855 & Other Effects & No & Yes \\
\hline Case \#855 & Sleep changes & Yes & No \\
\hline Case \#866 & Classification of Effects (Overlap with an Effect) & No & Yes \\
\hline Case \#866 & Evidence of Causality (Any comment) & No & Yes \\
\hline Case \#866 & Support and Advice (Any comment) & No & Yes \\
\hline Case \#880 & Classification of Effects (Overlap with an Effect) & No & Yes \\
\hline Case \#880 & Mental or mood changes & Yes & No \\
\hline Case \#880 & My Doctor (Any comment) & Yes & No \\
\hline Case \#880 & Nose Throat Chest & No & Yes \\
\hline Case \#888 & Mental or mood changes & Yes & No \\
\hline Case \#888 & My Doctor (Any comment) & No & Yes \\
\hline Case \#895 & Appetite and Weight & No & Yes \\
\hline Case \#895 & Other Effects & Yes & No \\
\hline Case \#898 & Classification of Effects (Overlap with an Effect) & No & Yes \\
\hline Case $\# 906$ & Mental or mood changes & No & Yes \\
\hline Case \#906 & Sleep changes & No & Yes \\
\hline Case \#921 & Assessing the Overall Experience & No & Yes \\
\hline Case $\# 938$ & Classification of Effects (Overlap with an Effect) & Yes & No \\
\hline Case \#967 & Friends and Family (Any comment) & No & Yes \\
\hline Case \#974 & Assessing the Overall Experience & Yes & No \\
\hline Case \#974 & My Doctor (Any comment) & Yes & No \\
\hline Case $\# 974$ & Other Drugs and Drug Combinations (Any comment) & No & Yes \\
\hline Case \#974 & Support and Advice (Any comment) & No & Yes \\
\hline Case $\# 975$ & Finances / The System (Any comment) & No & Yes \\
\hline Case $\# 992$ & Appetite and Weight & No & Yes \\
\hline Case \#992 & Assessing the Overall Experience & Yes & No \\
\hline
\end{tabular}




\begin{tabular}{|c|l|c|c|}
\hline Case & \multicolumn{1}{|c|}{ Code } & $\mathbf{1}^{\text {st }}$ coder & $\mathbf{2}^{\text {nd }}$ coder \\
\hline Case \#992 & Classification of Effects (Overlap with an Effect) & No & Yes \\
\hline Case \#992 & $\begin{array}{l}\text { Dealing with side effects / Finding optimum } \\
\text { effectiveness (Any comment) }\end{array}$ & Yes & No \\
\hline Case \#992 & Theories of Drug Action (Any comment) & Yes & No \\
\hline Case \#997 & Classification of Effects (Overlap with an Effect) & No & Yes \\
\hline Case \#997 & Evidence of Causality (Any comment) & Yes & No \\
\hline Case \#997 & Other Drug Issues (Any comment) & No & Yes \\
\hline Case \#997 & Pharmaceutical Companies / FDA & Yes & No \\
\hline
\end{tabular}




\section{APPENDIX H}

WikiScanner Search Results for Pharmaceutical Company Edits from 2002-2007

Astra Zeneca: Total edits: 949

Edits related to medications or pharmaceutical companies: 23 (2.4\%)

$\underline{\text { Wikipedia entries edited and a description of edits made }}$

- “Astra Zeneca": 6 edits

- Updated name of CEO, Vice President position title, web address, and similar

- "Fed Food, Drug, and Cosmetic Act": 3 edits

$\circ$ Added and edited statements about the history of the Act

- "FDA": 1 edit

○ Added entry to searchable category "pharmacology"

- "Good Manufacturing Practice": 4 edits

- Added and deleted sections about enforcement and purpose of guidelines

- “Omeprazole": 1 edit

- Deleted sentence: "Faced with the loss of patent protection and competition from generic manufacturers, AstraZeneca developed, launched, and heavily marketed [[esomeprazole]] (Nexium), a single [[enantiomer]] form of omeprazole”

- Added summaries of several studies where Nexium outperforms Prilosec

- "Quetiapine": 6 edits

- Edited 2 mistakes about dosages; edited language regarding approved indications 
○ Deleted sentence and reference for: "a known risk that teenagers taking the drug 'may be more likely to think about harming or killing themselves or to plan or try to do so"”

- "Risperidone": 1 edit

○ Deleted sentence: "Risperidone is now the most commonly prescribed [[antipsychotic]] medication in the [[United States]]"

- Note: Risperidone is made by Janssen

- "Thalidomide": 1 edit

$\circ$ Changed quotations around a phrase to parentheses

Eli Lilly: Total edits: 832

Edits related to medications or pharmaceutical companies: $38(4.6 \%)$

Wikipedia entries edited and a description of edits made

- "Dimethoxy-4-iodoamphetamine": 1 edit

- Described used of DOI in research

- "Cocaine": 1 edit

○ Deleted word "potent" before "SSRI" regarding Venlafaxine as a treatment for cocaine addiction

- "Drotrecogin alfa": 4 edits

○ Deleted word “deceptive" before "marketing campaign" regarding Lilly’s promotion of drug; Changed indication to state that a second opinion is not necessary 
O Added sentence, "PROWESS was terminated early for a statistically significant positive efficacy signal - unethical to continue trial - as this would have meant exposing severe sepsis patients to possibly receiving placebo."

○ Deleted 6 contraindications, including for pregnancy, breast feeding, and patients under age 18

- "Eli Lilly and Company": 13 edits

○ Corrected revenue figures from $\$ 12.6$ billion to $\$ 13.9 \mathrm{~b}$

- Added names of drugs to a list of Lilly's products; minor edits to title of former president Bush who was a board member

- “Erectile Dysfunction": 2 edits

- Added link to the Cialis home page

- "G D Searle and Company": 1 edit

○ Deleted statement that former CEO Donald Rumsfeld "could be the most prominent [[douchebag]] in the U.S."

- "Insulin/ Insulin analog": 6 edits

- Edited mode of production; corrected spelling of "lilly" and "lispro" (a drug)

- “Lilly Research Laboratories": 1 edit

○ Created page for above entry; added 1 introductory sentence

- “Novo Nordisk": 1 edit

○ Deleted "the first" regarding NovoLog being "the first rapid acting insulin analogue"

- "Olanzapine": 1 edit

- Corrected registered name of drug 
- "Pemetrexed": 1 edit

○ Added that drug is "clinically developed by Indianapolis based drug maker, Eli Lilly \& Company"

- "Pfizer": 2 edits

- Grammatical clean-up

- "Potassium bromide": 3 edits

- Added information about use for seizures in dogs

- Talk:Fluoxetine: 1 contribution

- Added paragraph: "I have been taking fluoxetine and had very little in the way of side effects - just a small amount of nausea at the start. It has been very successful in treating my depression so far and I'm feeling good for the first time in ages. I just wanted to add this as I'm sure there are others who it has helped and as mentioned above they don't tend to speak out. I think that an explanation in part of why there is such a broad range of reactions to drugs like these is the limited understanding of the basis of the disease. In my opinion what we know as depression could have many causes each presenting with similar symptoms explaining why some treatments only work for some individuals."

GlaxoSmithKline: Total edits: 1,148

Edits related to medications or pharmaceutical companies: $31(2.7 \%)$

$\underline{\text { Wikipedia entries edited and a description of edits made }}$

- "Celecoxib": 2 edits

- Corrected grammar 
- "Fluticasone": 4 edits

- Edited grammar and links

- "GlaxoSmithKline": 15 edits

○ Added paragraph that starts: "For many years now GSK has been a leading contributor to a multinational govenment and industry alliance to rid the world of lymphatic filariasis (elephantiasis)...." and added new subheading called "Good Works"

○ Minor edits to headquarters and locations; updated revenues (an increase) and number of employees (an increase); added links to GSK site for corporate governance, global locations and merger history

- "Lamotrigine": 2 edits

- Added and then deleted link to generic drug manufacturer

- "Lapatinib": 1 edit

○ Corrected name of drug

- "Motilin": 1 edit

- Elaborated on description of the hormone

- "Neuropathy": 1 edit

- Added word "central" to nervous system disorder

- "Resistin": 3 edits

- Minor edits to existing references for clinical studies

- Talk:GlaxoSmithKline: 1 edit

- Added statement that GSK still makes Ribena 
- Talk:Reverse transcriptase inhibitor: 1 edit

- Created talk page with: "Understanding RTI provide a novel platform for the developing of anti-HIV medication. This is one area that GlaxoSmithKline needs to be commended." 
VITA

\section{SHANNON HUGHES}

2002 BSW, Social Work

Florida State University

Tallahassee, FL

2002 Summer Fellow, Institute for Humane Studies

Philadelphia, PA

2002 Social Work Internship

Florida State Hospital

Chattahoochee, FL

2003 Mental Health Associate

Ten Broeck Hospital

Jacksonville, FL

2004 MSW, Social Work

Florida State University

Tallahassee, FL

2004 Social Work Internship; Assistant to Program Coordinator National HIV/AIDS Program

Tortola, British Virgin Islands

2005-2006 Executive Director

Jacksonville Real Estate Investors Association

Jacksonville, FL

2006-2010 Ph.D. Candidate in Social Welfare

Florida International University

Miami, FL

2008-2009 Instructor

Florida International University, School of Social Work

Miami, FL

2009-2010 Dissertation Year Fellowship

Florida International University

Miami, FL

2009-2010 Fahs-Beck Scholar

Fahs-Beck Fund for Research and Experimentation 


\section{PUBLICATIONS AND PRESENTATIONS}

Cohen, D., \& Hughes, S. What's in a "nonstimulant"? A preliminary review of published studies on Strattera (Atomoxetine). $7^{\text {th }}$ Annual Conference, International Center for the Study of Psychiatry and Psychology, Chicago, Illinois, Oct 4, 2003.

Hughes, S., \& Cohen, D. The construction of expertise in the age of the Internet:

Psychotropic drug knowledge in consumer-constructed online spaces. Medicine 2.0

Congress: Web 2.0 in Health and Medicine, Toronto, Canada, September 4-5, 2008.

Hughes, S., \& Cohen, D. (2009). A systematic review of long-term studies of drug treated and non-drug treated depression. Journal of Affective Disorders, 118(1), 9-18.

Cohen, D., Hughes, S., \& Jacobs, D. (2009). The deficiencies of drug treatment: The case of Strattera. In S. Timimi \& J. Leo (eds.), Rethinking ADHD: From brain to culture (pp. 313-333). New York: Palgrave Macmillan.

Lacasse, J.R., \& Hughes, S. Discordance between evidence-based neuroscience and direct-to-consumer advertising of antidepressants on the world wide web: A five-year follow-up. Society for Social Work and Research, San Francisco, California, January 14$17,2010$.

Hughes, S., \& Cohen, D. (2010). Understanding the assessment of psychotropic drug harms in clinical trials to improve social workers' role in medication monitoring. Social Work, 55(2), 105-115. 